Journal of Clinical Investigation

Vol. 41, No. 6, 1962

\title{
PROCEEDINGS OF THE FIFTY-FOURTH ANNUAL MEETING OF THE AMERI- CAN SOCIETY FOR CLINICAL INVESTIGATION, INC., HELD IN ATLANTIC CITY, N. J., APRIL 30, 1962
}

\author{
Presidential Address \\ THE TRAINING OF THE CLINICAL INVESTIGATOR
}

BY HENRY G. KUNKEL

In this address, I would like to touch on a few facets of the intricate problem of training clinical investigators. During the past year the Society has received several requests from representatives of different branches of the Public Health Service for aid in the evaluation of the various training-grant programs as they apply to clinical investigation. Several discussions have been held with representatives of the Society with the expected result that no one could clearly define an optimal program of training for such a variegated individual as a clinical investigator. The question arose and of course will long continue to arise: Should clinical investigators train with clinical investigators or should they work with more basically oriented scientists during their training period?

However, before going farther, I would like to tell a little of the story of Doctor Brightfool. He was a most highly trained doctor, senior resident in medicine at the main teaching hospital attached to Harvumbia Medical School. His special forte was new steroid hormones and none could rival him in his knowledge of their dosages. Naturally, he became curious about their mechanism of action, but unfortunately he was far too busy in the conscientious pursuit of his hospital duties to even consider attempting any experiments. Suddenly, in a moment of exhilaration, he made the momentous decision to go into clinical investigation and tackle the steroid problem so dear to his heart. Of course he was no little swayed by an innate conviction that "research accomplishment is the royal road to academic success." His joy knew no bounds when he was accepted as a research associate at the Federal Institute for the Preservation of Health. There was no longer any doubt in his mind; he was determined to become an academic medicine man. The day finally arrived when he tried his first experiment in the laboratory. Many were his frustrations, and how he wished he knew more chemistry, or even as much as he had once known. It soon became apparent that all he needed was more basic training in enzyme chemistry. $\mathrm{He}$ was with too clinical a group. So after two years he left and went back to the biochemistry department at his own medical school, where he was given the problem of purifying the enzyme hydroxymethyl deoxycytidine monophosphate kinase. It was a slow process but he stuck it out because he felt he was really learning enzyme chemistry. Eventually he ended up in a joint appointment with the department of medicine, but by now he knew much more about kinetics than corticoids. Things looked rather bleak after some five years of such combined activity and it gradually dawned on him that he was not really cut out to be an investigator. Fortunately, rescue did occur when he was suddenly appointed director of a small new Institute for the Study of Cancer, Arthritis, Arteriosclerosis and Allied Disorders (the latter referring to other diseases sponsored by wealthy foundations). He was now content-he had found his calling - he had become an administrator, which of course is where (according to Parkinson's law) he would have ended up anyway, even if he had been a good investigator. So what did it matter?

Unfortunately, this little tale is not pure fantasy but a sequence of events observed not infrequently in the area of clinical investigation. The great tragedy in it is the loss of highly trained physicians from vocations where they are urgently needed. It has been estimated that the training of a good physician cost something of the order of $\$ 50,000$. This large sum, which represents only tangible costs, plus the added expense of special training, emphasizes numerically the tragedy involved when the end result is simply a poor investigator. Then there is the important question of the shortage of scientific manpower, which is exemplified by weekly advertisements in The New York Times for mathematicians and physicists (up to now there has been none for clinical investigators). This is reflected in the alarming fall-off in the number of applicants to medical schools, and makes it all the more imperative that the medical school commodity be put to the right use.

With so much at stake, it would seem of the utmost importance that the skilled physician should have some evidence of an aptitude for investigative work before taking the drastic step of enrolling in a prolonged plan of training in so different an area. Whenever possible, this should be ascertained at an early period, preferably during the resident training period or even in medical school. Perhaps greater freedom from an oppressive duty load during the house officer training period, as is frequently advocated but rarely enforced, would represent one way to permit the inquiring individual an earlier opportunity to test, at least in a preliminary way, his inclinations and even fitness for the research alternative. Interest and enthusiasm are scarcely sufficient without other attributes for a truly successful career in clinical investigation. Few individuals possess the insight to admit to ineptitude, especially since proficiency in this area is so intangible. It is too easy to justify failure with plausible reasons such as the vicissitudes of research work 
or inadequate training. Scientific inquiry is a sort of opiate that once experienced is not readily shaken off, particularly if the individual has tasted what has been termed "the Elysian delight" of observing something new.

The competitive criteria generally regulating advancement in most scientific fields are not so clearly operative in the area of clinical investigation, particularly in the present climate of plentiful funds and expanding clinical research centers. Sufficient opportunity exists to encourage even the poor investigator to persist in his course at relatively little self-sacrifice. In the long run, the burden of responsibility for the important decision of whether the young man stays in clinical investigation must fall primarily on the shoulders of the established investigator who has observed him in action. This very difficult yet essential assignment requires considerable thought and might well be considered an obligation to the young associate, although help and advice on this question are rarely sought.

There is no doubt that a place will always remain for the untrained individual with ingenuity and keen observational ability. It is almost stating a truism to say that these talents can never be replaced by simple experience with highly specialized techniques. However, neither should the latter be avoided. The British geneticist, Darlington, has recently presented a strong indictment of medical training for failing to provide the foundation for understanding the advances in biology, and he cites an example as follows: "Chromosomes which have been understood in agricultural research for forty years made their debut in medical research only four years agoand, as far as medical teaching is concerned, the chromosomes still exist only on paper."

Many of our distinguished predecessors in this Society availed themselves of opportunities to work with prominent scientists abroad for variable periods of time after their clinical training. This has become less popular in recent years but still remains a valuable opportunity, particularly in certain fields. In most instances the young physician can readily find the facilities for further basic training within the confines of his own university or medical school. Our research institutes can also play a useful accessory role. Theobald Smith once stated that each new generation of "research workers must have more diversified training than the older generation possesses." Can this be acquired by physicians working solely in clinical investigation laboratories? Facts are continually accumulating in many branches of science and in obscure areas that have application to human disease but which are unfamiliar to most of the present generation of clinical research workers. Our training and background do not permit us to see or appreciate their relevance, and it is highly desirable that the young investigator have an opportunity to explore at least a few of these areas during his training period.

In addition, there is an element of selfishness in this era of large groups and teams where the project represents the first consideration and the training of the young member much the second. The attitude that what is good for the project is good for the younger individual in it is not uncommon. With the effective sterilization of our selected best investigators by a continuous series of committee meetings, conferences, and symposia, an ever increasing responsibility for actual investigative work falls to the junior members of the research team. All too frequently they do not represent co-workers acquiring experience and training, working shoulder to shoulder with the established investigator, observing his approach, technique, and fulfillment of a problem. Instead they are hard workers struggling on their own, and this is important, not with their own approaches but with the projected plan of the senior investigator. He still manages to find the time to fill the junior worker with ideas and schemes and then to report the experimental findings at select conferences where the junior member, unfortunately, happens not to be invited. This of course is an exaggeration, but the key question that must be faced is: How often is the senior investigator prepared to advise his junior associates to leave their essential position on his team and seek more basic training in laboratories which might furnish them with new, difficultly acquired techniques and fresh perspectives to apply to clinical problems?

There are of course a number of dangers that arise in the instances where very thorough grounding in the basic sciences is undertaken. Perhaps foremost of these is the very real problem of the almost invariable sacrifice of proficiencies in clinical medicine which remain such an essential ingredient of the clinical investigator, at least in the classical sense. Much has been said on this point. There is also little doubt that clinical investigation needs at least some basically trained individuals. Perhaps ideally it should encompass a whole spectrum of investigators operating at various distances from the bedside.

Another very real danger is that the physician undertaking prolonged basic training may be lost completely to clinical investigation. He may well become so enamored of fundamental problems of biology as to forsake entirely his disease orientation. In addition, he almost certainly will encounter the rather widespread disparaging attitude of basic scientists toward clinical investigation which dampens the original appeal of a career in this area. Such individuals, particularly those participating in various postdoctoral Ph.D. programs, are in considerable competitive demand from various branches of the preclinical sciences where they frequently end up in important positions, all connections with clinical investigation severed. Perhaps this is an overstatement; one link with clinical investigation often does remain and that is membership in this Society.

Here then, to digress for a moment, is one of the factors behind our current Society dilemma. These individuals and other full-time research workers, perhaps somewhat less removed from clinical investigation, represent an ever expanding group. Membership in the Society is readily gained under the present system where research accomplishment is the dominant criterion for admission. However, the important point is that it is gained at the expense of the classical type of clinical in- 
vestigator who is involved in patient care. Under our present fixed quota system, someone must lose out. It is imperative that steps be taken to prevent this selective action on the more clinically oriented individuals who in the past have represented the backbone of the Society. The most ready remedy, although no solution, is to support the proposed increase in membership.

There is one final important point regarding the education of the clinical investigator about which relatively little is heard. This concerns training in what might be termed the philosophy of research and involves such diverse questions as scientific discipline of thought, intellectual integrity, the sanctity of the written word, and even the ethics of research work. Somehow, medical training does not regularly inculcate upon the investigator the basic principles involved in these more esoteric questions. Their acquisition is often a slow, difficult process accomplished primarily through bitter experience, and others must suffer while the young worker learns by his mistakes. It is an area where the physician most certainly requires development, and this then marks his transition into a scientist. Irrespective of the type of training program adopted by a clinical investigator, be it in part-time clinical research or in basic science laboratories, or perhaps ideally in both, strong consideration should also be given to the acquisition of this other, most essential type of education. 
Journal of Clinical Investigation

Vol. 41, No. 6, 1962

\section{PAPERS PRESENTED AT THE FIFTY-FOURTH ANNUAL MEETING 1962}

1. Role of the Thymus in Development of Immunity. Robert A. Good, ${ }^{*}$ Carlos Martinez, Olga K. Archer and Ben W. Papermaster, Minneapolis, Minn. (1361)

2. Studies of an Antigen in Cell Walls of Group A Streptococci Possessing an Immunologic Relationship to Human Heart Tissue. Melvin H. Kaplan, ${ }^{*}$ Cleveland, Ohio. (1370)

3. Effects of Humoral Factors on Antigenic Histamine Released from Human Leukocytes. Paul P. VAN Arsdel, JR., Seattle, Wash. (introduced by Clement A. Finch). (1407)

4. Reticuloendothelial Clearance of Circulating Fibrin in the Pathogenesis of the Generalized Shwartzman Reaction. Leung Lee, New York, N. Y. (introduced by Chandler A. Stetson). (1378)

5. The Heterogeneity of Particulate Fat in Plasma. Edwin L. Bierman, Enoch Gordis and James T. Hamlin, III, New York, N. Y. (introduced by Vincent P. Dole). (1345)

6. Studies on the Relationship of Angiotensin to Hypertension of Renal Origin. Russell E. Morris, Jr., Patricia A. Ransom and John Eager Howard, Baltimore, Md. (introduced by Samuel P. Asper, Jr.). (1386)

7. Angiotensin II and Renal Sodium Transport: Natriuresis and Diuresis in Patients with Cirrhosis and Ascites. John H. Laragh, ${ }^{*}$ Paul J. Cannon, Richard P. Ames, Alfred M. Sicinski, Carl J. Bentzel and Jay I. Meltzer, New York, N. Y. (1375)

8. The Importance of Conjugation with Glutathione on Sulfobromophthalein (BSP) Transport from Blood to Bile. Burton Combes, Dallas, Tex. (introduced by Gladys Fashena). (1351)

9. Primary Hyperoxaluria: A Defect in Glyoxylate Metabolism. Elizabeth W. Frederick, Mitchell T. Rabkin and Lloyd H. Smith, Jr., ${ }^{*}$ Boston, Mass. (1358)

10. A Mechanism of Action of Colchicine in Acute Gouty Arthritis. J. E. Seegmiller, R. Rodney Howell and Stephen E. Malawista, Bethesda, Md. (introduced by Gordon M. Tomkins). (1399)

11. Pseudofeedback Inhibition of Purine Synthesis by 6-Mercaptopurine and Other Purine Analogues. Robert J. McCollister, Walter R. Gilbert, Jr. and James B. Wyngaarden, ${ }^{*}$ Durham, N. C. (1383)

12. Characteristics of the Genetic Code. Oliver W. Jones, J. Heinrich Matthaei and Marshall IV. Nirenberg, Bethesda, Md. (introduced by Ivan W. Brown, Jr.). (1369)

* Member.

( ) Page number of abstract.
13. Abnormal Myoglobin Chromatography in Childhood Muscular Dystrophy. Gerald T. Perkoff, ${ }^{*}$ Robert L. Hill and Frank H. Tyler, ${ }^{*}$ Salt Lake City, Utah and Durham, N. C. (1391)

14. The Regulation of Spleen Growth and Sequestering Function. Harry S. Jacob, Richard A. MacDonald and James H. JANDL, ${ }^{*}$ Boston, Mass. (1367)

15. The Structural Basis for the Action of Neurohypophyseal Hormones upon the Amphibian Urinary Bladder. Howard Rasmussen* and IRving L. Schwartz, ${ }^{*}$ Madison, Wis. and Cincinnati, Ohio. (1393)

16. Measurements of $\mathrm{K}^{+}$Exchange in Separated Renal Tubules. Maurice Burg, Mones Berman and JACK Orloff, ${ }^{*}$ Bethesda, Md. (1349)

17. The Effect of Renal Perfusion Pressure on the Active Transport of Sodium out of Distal Tubular Urine as Studied with the "Stop-Flow" Technique. LouIS Tobian,* Karen Coffee, Dorothy Ferreira and Judith Meuli, Minneapolis, Minn. (1404)

18. Competitive Inhibition of Dibasic Amino Acid Transport in Rat Kidney. Leon E. Rosenberg and Stanton Segal, Bethesda, Md. (introduced by Robert M. Chanock). (1394)

19. Periodic Hemodialysis for the Rehabilitation of Patients with Terminal Uremia. Belding H. ScribNER, ${ }^{*}$ Seattle, Wash. (1398)

20. The Use of Synchronized Direct-current Countershock in the Treatment of Cardiac Arrhythmias. BERNARD Lown, Raghavan Amarasingham, Jose Neuman and Barouh Berkovitz, Boston, Mass. (introduced by Samuel A. Levine). (1381)

21. Myocardial Metabolism during Epinephrine-induced Necrosis. Timothy J. Regan, LaWrence Trovm, Patrick H. Lehan and Harper K. Hellems, ${ }^{*}$ Jersey City, N. J. (1393)

22. Studies on the Pathogenesis and Clinical Features of "Alcoholic Hypoglycemia." N. Freinkel,* D. L. Singer, C. K. Silbert and J. B. Anderson, Boston, Mass. (1359)

23. Retardation of Sodium Flux in Dog Erythrocytes by Physiological Concentrations of Aldosterone In Vitro. David H. P. Streeten and Colette Spach, Syracuse, N. Y. (introduced by Eugene L. Lozner). (1403)

24. The Secretion of 18-Dihydroaldosterone by the Human Adrenal. Stanley Ulick and Kathryn Kusch Vetter, New York, N. Y. (introduced by Solomon A. Berson). (1406)

25. The Role of Endogenous Glucagon in Blood Glucose Regulation in Man. Roger H. Unger and Anna M. Eisentraut, Dallas, Tex. (introduced by Elias Strauss). (1406)

26. The Hypoglycemic Effect of Ketone Bodies. David Mebane and Leonard L. Madison,* Dallas, Tex. (1383) 


\section{SECTIONAL MEETINGS}

Section I: INFECTIOUS DISEASE AND IMMUNOLOGY

1. Hypogammaglobulinemia produced by the Administration of 6-Thioguanine to Patients with Nephrosis. Sheldon M. WolfF and Howard C. Goodman, Bethesda, Md. (introduced by James H. Baxter). ASCI (1413)

2. Measurement of Hemolytic Complement ( $\left.C^{\prime}\right)$ in Synovial Fluids. T. Pekin and N. J. Zwaifler, Washington, D. C. AFCR

3. The Mechanism of Death in Experimental Pneumococcal Infection. John E. Perry and Leighton E. ClufF*, Baltimore, Md. ASCI (1392)

4. Increased Peripheral Resistance in Patients with Septic Shock. V. N. UdhojI, M. H. Weil, M. P. Sambir and R. L. Sudrann, Los Angeles, Calif. AFCR

5. Impaired Leukocytic Bactericidal Activity Resulting from Cortisol Treatment. F. Allison and M. $\mathrm{H}$. ADCock, Jackson, Miss. AFCR

6. Studies on Lysosomes. The Effect of Cortisone and Endotoxin. Gerald Weissmann and Lewis ThomAs, ${ }^{*}$ New York, N. Y. ASCI (1410)

7. Susceptibility to Infection in Experimental Anemia. D. KAYE and E. W. Hook, New York, N. Y. AFCR

8. Selective Inhibition of Enterovirus Nucleic Acid Synthesis by 2 -( $\alpha$-Hydroxybenzyl)-Benzimidazole (HBB). Hans J. Eggers and IgOr Tamm,* New York, N. Y. ASCI (1356)

Section II : ENDOCRINOLOGY

1. Metabolic Abnormalities of Adipose Tissue from Patients with Early Diabetes. J. A. Owen, J. H. Gaskin, W. D. Warren and G. Hollifield, Charlottesville, Va. AFCR

2. Studies on the Mechanism of Ethanol-induced Hypoglycemia. James B. Field* and Hibbard E. WilLIAMS, Bethesda, Md. ASCI (1357)

3. Studies to Determine whether Glucose Must Be Metabolized to Induce Insulin Release. Charles Kilo, Charles L. Long, Jr., Rita M. Bailey, Mary B. Koch and Lillian Recant, ${ }^{*}$ St. Louis, Mo. ASCI (1372)

4. An In Vitro Influence of the Central Nervous System Tissues on Thyrotropin Secretion. W. D. OdELl, Bethesda, Md. AFCR

5. Studies on the Role of the Brain in the Steroidal Inhibition of ACTH Release. JoHN W. KENDALL, Kunio Matsuda, Caroline Duyck and Monte A. Greer,* Portland, Ore. ASCI (1371)

6. Impaired Aldosterone Production in "Salt-losing" Congenital Adrenal Hyperplasia. G. T. Bryan, B. Kliman and F. C. Bartter, Bethesda, Md. AFCR

7. Urinary $\Delta$-5-Pregnene- $3 \beta, 17 \alpha, 20 \alpha, 21$-Tetrol in $3 \beta$-olDehydrogenase Deficiency. Alfred M. Bongiovanni* and Albert Clark, Philadelphia, Pa. and Montreal, Canada. ASCI (1346)
8. Biologic Potential of Transcortin. Avery A. SANDBerG* and W. R. Slaunwhite, Jr., Buffalo, N. Y. ASCI (1396)

9. Corticotropin and Gonadotropin Effect on the Biosynthesis and Catabolism of Steroids Derived from Virilizing Ovarian Tumors. A. L. ENNIS and S. Richardson Hill, Jr., Birmingham, Ala. AFCR

\section{Section III : METABOLISM}

1. The Effect of the Physician's Role upon Patient Response to an Unpleasant Task. M. D. Bogdonoff, L. BREHM and K. BACH, Durham, N. C. AFCR

2. Abnormal Neural Regulation of Vasopressin and Aldosterone Secretion in a Patient with Cerebral Hyponatremia. Joseph F. Dingman, Akira ArImura, Ralph E. Peterson* and Robert G. Heath, Boston, Mass., New York, N. Y. and New Orleans, La. ASCI (1354)

3. A Syndrome of Periodic Hypothalamic Discharge. S. M. Wolff, R. C. Adler, E. R. Buskirk and R. H. Thompson, Bethesda, Md. AFCR

4. In Vivo and In Vitro Studies of Effect of L-Leucine on Carbohydrate Metabolism. R. A. Yerry, S. Pensuwan, F. A. Zacharewicz and T. F. Frawley, Albany, N. Y. AFCR

5. Observations on Cataract Formation in the Newborn of Pregnant Rats Fed a High Galactose Diet. Stanton Segal and Howard Bernstein, Bethesda, Md. (introduced by Jacob Robbins). ACSI (1399)

6. Site of Action of Androgen on Adrenal Steroidogenesis. Julian I. Kitay, Charlottesville, Va. (introduced by Alfred Jay Bollet). ASCI (1373)

7. Incorporation of 17-Hydroxy-Pregnenolone- $\mathrm{H}^{3}$ into Cortisol by the Human Adrenal In Vitro and by the Dog Adrenal In Vivo. Patrick J. Mulrow, George L. Cohn, Albert A. Kuljian and William F. Ganong, New Haven, Conn. and San Francisco, (introduced by Philip K. Bondy). ASCI (1388)

8. Synthesis of Fatty Acids by Mitochondria. W. R. Harlan, S. Boone and S. C. Dees, Durham, N. C. AFCR

\section{Section IV : KIDNEY AND ELECTROLYTES}

1. The Relationship between Electrical and Hydrogen Ion Gradients Across the Rat Proximal Tubule. N. BANK, New York, N. Y. AFCR

2. A Kinetic Analysis of the Antidiuretic Action of Vasopressin. Isidore S. Edelman* and Martin J. PeterSEN, San Francisco, Calif. ASCI (1356)

3. Water-Water Interaction during Solvent Flow in Isolated Turtle Bladders. William A. Brodsky* and Theodore P. Schilb, Louisville, Ky. ASCI (1348)

4. Osmotic Water Transfer Across a Vasopressin-sensitive and an Artificial Membrane. Laurence E. Earley, Boston, Mass. (introduced by Laurence B. Ellis). ASCI (1355) 
5. The Effect of Adenosine Derivatives on Ion Transport and Energy Metabolism in the Toad Bladder. RoBert P. Davis, Mitzy Canessa-Fischer and Chester M. Edelmann, JR., New York, N. Y. (introduced by I. Herbert Scheinberg). ASCI (1353)

6. The Action of Streptolysin S on Renal Basement Membrane. E. M. TAN and M. H. Kaplan, Cleveland, Ohio. AFCR

7. Osmolality, $\mathrm{pH}$, and Inulin Concentration in Proximal Tubular Fluid of the Dog. James R. Clapp and John F. Watson, Bethesda, Md. (introduced by Robert W. Berliner). ASCI (1350)

8. An Assay for ADH in which Levels Were Demonstrated in Jugular Vein Plasma of Children with Nephrogenic Diabetes Insipidus (NDI). M. A. Holliday, C. Burstin, L. TaVoularis and L. Poppe, Pittsburgh, Pa. AFCR

\section{Section V: Gastroenterology}

1. Effect of Ammonium Chloride and Urea Infusions on Gastric Ammonium Levels. B. Fleshler and G. Gabuzda, Cleveland, Ohio. AFCR

2. Effects of Norepinephrine on Hepatic Circulation in Dogs. F. A. Bashour and D. P. Sellers, Dallas, Tex. AFCR

3. Mechanism of Bilirubin Excretion by the Avian Kidney. E. E. Owen, I. Williamson and R. R. Robinson, Durham, N. C. AFCR

4. Abnormal Rise of Plasma Amino Acids and the Appearance of a Polypeptide in the Plasma in Adult Celiac Disease after Oral Administration of Gliadin. O. Dhodanand Kowlessar, Elliot Weser and Lorraine J. Haeffner, New York, N. Y. (introduced by Marvin H. Sleisenger). ASCI (1374)

5. Adult Celiac Disease: Rapid Sequential Changes in Jejunal Mucosa with Alterations of Dietary Gluten. Theodore M. Bayless, John H. Yardley, James H. Norton and Thomas R. Hendrix, Baltimore, Md. (introduced by Ivan L. Bennett, Jr.). ASCI (1344)

6. The $\mathrm{I}^{131}$-triolein Absorption Test. N. TunA, H. K. Mangold and D. Mosser, Minneapolis, Minn. AFCR

7. A Novel Metabolic Pathway of Fat Absorption in the Intestinal Mucosa. John R. Senior and Kurt J. IsSElbaCher, ${ }^{*}$ Boston, Mass. ASCI (1399)

8. Direct Evidence for the Participation of Endogeneous Lipids in the Formation of Dietary Chylomicrons. Samuel J. Friedberg, Morton D. Bogdonoff, E. Harvey Estes, Jr., Paul B. Sher and Gitta Jackson, Durham, N. C. (introduced by David T. Smith). ASCI (1359)

\section{Section VI : hEMATOLOGY}

1. Relationship of High Levels of "Bart's" Hemoglobin in Infancy to $\alpha$-Thalassemia. David J. Weatherall, Baltimore, Md. (introduced by C. Lockard Conley). ASCI (1409)

2. Evidence for a Second Iron-binding System in Plasma. Raymond J. Dern, Alberto Monti and Michael F. Glynn, Chicago, Ill. (introduced by Richard L. Landau). ASCI (1354)
3. Initiation of the Action of Complement in a Human Autoimmune Hemolytic System, the Donath-Landsteiner Reaction. CarL F. Hinz, Jr.* and Anna Marie Mollner, Cleveland, Ohio. ASCI (1365)

4. Correlation of Complement-fixing Antibodies with Platelet Survival in Thrombocytopenic Patients. R. H. Aster, R. H. Levin, H. E. Cooper, E. J. Freireich and E. E. Morse, Bethesda, Md. AFCR

5. Biochemical Interaction between Two Populations of Red Cells. E. Beutler and M. C. Baluda, Duarte, Calif. AFCR

6. Genetic Implications of Diisopropyl Fluorophosphate (DFP) ${ }^{32}$-Erythrocyte Survival Studies in Negro Females Heterozygous for Glucose-6-Phosphate Dehydrogenase Deficiency. George J. Brewer, ALvin R. Tarlov and Robin D. Powell, Chicago, Ill. (introduced by Alf S. Alving). ASCI (1348)

7. A New Method for Measuring Minimum In Vivo Concentrations of Factor VIII Applied in Distribution and Survival Studies. N. Raphatel Shulman,* Victor J. Marder and Merilyn C. Hiller, Bethesda, Md. ASCI (1401)

8. Different Patterns of Hematologic Remissions in Acute Myelocytic Leukemia. R. H. Levin, G. M. Brittin and E. J. Freireich, Bethesda, Md. AFCR

\section{Section VII : CARDIOPULMONARY}

1. An Early Pulmonary Physiologic Abnormality in Progressive Systemic Sclerosis (Diffuse Scleroderma). R. J. Wilson, G. P. Rodnan and E. D. Robin, Pittsburgh, Pa. AFCR

2. Functional Characteristics of the Left Atrium in Man. R. R. Johnston, C. Rackley, H. J. Sauter and H. T. Dodge, Seattle, Wash. AFCR

3. Nucleic Acid Studies in Experimental Cardiomegaly. R. G. Sumner and H. D. McIntosh, Durham, N. E. AFCR

4. Electrocardiographic and Hemodynamic Observations during Selective Coronary Cineangiocardiography. Richard S. Ross, Neil Schwartz, Robert A. Gaertner and Gottlieb C. Friesinger, Baltimore, Md. (introduced by A. McGehee Harvey). ASCI (1395)

5. Congenital Deafness, ECG Abnormalities and Sudden Death-A Recessive Syndrome. G. R. Fraser and P. Froggatt, Seattle, Wash. AFCR

6. Metabolism of Arterioles and Venules as Studied in the Cartesian Diver. Rufus O. Howard, David W. Richardson, Morris H. Smith, JR. and John L. Patterson, JR., * Richmond, Va. ASCI (1366)

7. Postural Hypotension in Wernicke's Disease: A Manifestation of Autonomic Nervous System Involvement. R. I. Birchfield, Seattle, Wash. AFCR

8. Diminished Biological Half-life of Angiotensin II in Hypertensive Plasma. Roger B. Hickler, David P. Lauler and George W. Thorn, ${ }^{*}$ Boston, Mass. ASCI (1365)

9. Antihypertensive Principle from Renal Medulla. E. E. Muirhead, J. W. Hinman, E. G. Daniels, M. Kosinski and B. Brooks, Detroit and Kalamazoo, Mich. ASCI (1387) 


\section{ABSTRACTS}

Effect of Reserpine on Vascular Responses of Forearm to Tyramine and Norepinephrine in Man. FrancoIs M. Abboud and John W. Eckstein, Iowa City, Iowa (introduced by Willis M. Fowler).

The vasoconstrictor response to tyramine is reduced, and that to norepinephrine is enhanced, in animals treated with reserpine. These effects have not been demonstrated in man. Observations on blood pressure (cuff) and forearm blood flow (plethysmograph) were made in 7 healthy men before and after intravenous injection of tyramine $(0.1 \mathrm{mg} / \mathrm{kg})$ and before and during 6-minute infusions of norepinephrine in doses of $0.032,0.064$, and $0.15 \mu \mathrm{g}$ per $\mathrm{kg}$ per minute. Each subject was studied 3 times. Session A served as the control. Session B followed 2 weeks of treatment with reserpine, $1 \mathrm{mg}$ daily. Session $C$ was held 3 to 6 weeks after reserpine was discontinued. Analysis of variance of the data revealed no significant differences in the levels of blood pressure, blood flow, or resistance to flow during norepinephrine infusions in the 3 sessions. The values for pressure, flow, and resistance obtained with all 3 doses of norepinephrine averaged $109 \mathrm{~mm}$ $\mathrm{Hg}, 3.39 \mathrm{ml}$ per minute per $100 \mathrm{ml}$ of forearm, and $33.5 \mathrm{U}$, respectively, during session $\mathrm{A}$. The corresponding values during session $B$ were $107 \mathrm{~mm} \mathrm{Hg}$, $3.26 \mathrm{ml}$, and $38.7 \mathrm{U}$. During session $\mathrm{C}$ these values were $111 \mathrm{~mm} \mathrm{Hg}, 2.69 \mathrm{ml}$, and $44.2 \mathrm{U}$. Resting levels of blood pressure and heart rate were slightly reduced in session $B$ while resistance was unchanged. The pressor response to tyramine during session B (4.3 \pm $0.9 \mathrm{~mm} \mathrm{Hg}$ ) was significantly lower than during session A $(13.7 \pm 4.1 \mathrm{~mm} \mathrm{Hg})$ or session $\mathrm{C}(21.0 \pm 3.77 \mathrm{~mm}$ $\mathrm{Hg}$ ). The responses to norepinephrine were similar in 5 additional subjects who received reserpine, $3 \mathrm{mg}$ daily for 5 days. Conclusion: In these experiments reserpine failed to cause vascular supersensitivity to norepinephrine even though the pressor response to tyramine was reduced.

Effect of Hypertension on Cholesterol Synthesis in Rats. Harold Adel, Marie M. Daly, Quentin B. Deming,* Lili Brun and Victoria Raeff, New York, N. Y.

The development of atherosclerosis in animals and man is accelerated by hypertension. Increased concentrations of cholesterol in serum and tissues have been observed in hypertensive rats. In order to determine whether these observations might be related to an effect of hypertension on cholesterol synthesis, the incorporation of labeled precursors into cholesterol by tissues of normotensive and Goldblatt-hypertensive rats was measured in rivo and in vitro. After intraperitoneal injec-

* Member. tion of acetate-1- $\mathrm{C}^{14}$ into hypertensive rats and paired normotensive controls, the cholesterol isolated from liver, serum, and residual carcass showed higher specific activity in the hypertensives. The mean increase was, in liver $58 \%$, in serum $57 \%$, in carcass $35 \%$; $\mathrm{p}$, respectively, less than $0.01,0.05,0.05$. The incorporation of acetate-1- $\mathrm{C}^{14}$ into cholesterol by liver slices was greater in slices from hypertensive animals than in those from paired normotensive controls. The mean difference in specific activity was $135 \%$ ( $p$ less than 0.005 ). No difference was shown when mevalonate-2- $\mathrm{C}^{\mathbf{1 4}}$ was used as precursor. The incorporation of acetate or mevalonate into cholesterol by 6 pools of aortas isolated from hypertensive rats and pools of paired normotensive controls was measured. With either precursor the incorporation of label into cholesterol (purified by repeated bromination) was higher in the aortas of the hypertensives ( $p$ less than 0.025 ). Synthesis of cholesterol, whether measured in the living rat or in isolated liver or aorta, is accelerated by hypertension.

The Mechanism of Puromycin Inhibition of Hemoglobin Synthesis by Rabbit Reticulocyte Ribosomes. DAvid W. Allen and Paul C. Zamecnik,* Boston, Mass.

Reticulocyte ribosomes contain the partially formed polypeptide chains of hemoglobin, of varying chain lengths but each having the same $\mathrm{N}$-terminal group, valine. These incomplete polypeptide chains are considered bound to the ribosome by soluble RNA residues attached to the peptide chain at its growing C-terminal end. Puromycin, a potent inhibitor of protein synthesis, is an analog of aminoacyl-soluble RNA. The effect of this drug on reticulocyte ribosomes, labeled with $\mathrm{C}^{14}$ amino acids by prior incubation of the intact reticulocytes, has been studied in a cell-free system capable of hemoglobin synthesis. Puromycin is found to release the polypeptide intermediates of hemoglobin synthesis from the ribosome. Unlike the more naturally occurring release of the completed hemoglobin molecule, the puromycin-produced release of polypeptide from the ribosome does not require an ATP-generating system or the supernatant fraction and occurs maximally in 1 or 2 minutes. The polypeptides released by puromycin do not appear as hemoglobin chromatographically or electrophoretically but, like the completed molecule, have only $\mathrm{N}$-terminal valine. $\mathrm{C}^{16}$-puromycin synthesized by methylating its tyrosyl analog with $\mathrm{C}^{\text {it }}$-diazomethane is bound to the soluble polypeptide it releases. Saturation of the binding site of the puromycin-released polypeptide occurs at a puromycin concentration (200 $\mathrm{m} \mu \mathrm{moles} / \mathrm{ml}$ ) equivalent to that producing maximal inhibition of $\mathrm{C}^{16}$-amino acid incorporation in a cell-free system and maximal release of $\mathrm{C}^{16}$-polypeptide from the ribosomes. One residue of puromycin is bound to the 
released polypeptide for every $\mathrm{N}$-terminal valine or one residue per polypeptide chain. It is concluded that puromycin inhibits protein synthesis by displacing the soluble RNA residues binding the chain to the ribosome and releasing the incomplete peptide chain. It appears possible to employ puromycin, during the course of synthesis of a given protein molecule, to obtain nascent peptides of varying chain length.

Leukocyte Alkaline Phosphatase in Mongolism; a Possible Chromosome Marker. Aaron A. Alter, Stanley L. Lee, Mohamed Pourfar and Gerald Dobkin, Brooklyn, N. Y. (introduced by Ludwig W. Eichna).

Polymorphonuclear leukocytes contain one or more phosphomonoesterases active at $\mathrm{pH}$ 9.3. In chronic granulocytic leukemia the activity of this enzyme(s) is reduced. In leukocytes of chronic granulocytic leukemia, absence of part of the long arm of one of the short acrocentric chromosomes has been observed. These two observations may be related: the missing chromosomal portion may contain the gene for the lacking enzyme. It is generally recognized that mongolism is associated with trisomy of a short acrocentric chromosome. If the same chromosome is involved in mongolism as in chronic granulocytic leukemia, an effect of the increased dosage of the gene might be evident in increased leukocyte phosphatase activity. Leukocyte phosphatase activity at $\mathrm{pH} 9.3$ was determined in a group of 36 healthy children with mongolism and in 35 healthy control children comparable in age. A mean value of $64 \mathrm{U}$ ( $\mathrm{mg}$ phosphorus $/ 10^{10}$ leukocytes in 1 hour) was found in the mongols; the control value was $24 U(p<0.001)$. When these values are expressed per $10^{10}$ polymorphonuclear neutrophils instead of total leukocytes, the mongols showed $139 \mathrm{U}$ and the controls 83 (mean values). This difference is statistically significant at the $1 \%$ level of confidence. These findings are consistent with the hypothesis that leukocyte alkaline phosphatase production is controlled by a gene on the chromosome for which mongols are trisomic. The ratio of $139 / 83$ of the phosphatase values approximates the $3 / 2$ that might theoretically be expected. Other explanations for the observed phosphatase elevation in the leukocytes of children with mongolism are, of course, possible.

Functional Differences in Lactic Dehydrogenase Activity as a Metabolic Basis for Cell Survival during $\mathrm{Hy}$ poxia. C. R. Amarasingham, G. M. Green and K. L. Stuart, Boston, Mass. (introduced by Edward H. Kass).

Although isoenzymes of different tissue origin are well characterized, the functional meaning of differences in enzymic structure are not well understood. Lactic dehydrogenase (LDH) with different physiochemical properties may be extracted from mammalian tissues. At least two different general molecular species of LDH clearly exist; there may be three additional intermediate forms, although in many tissues intermediate patterns of $\mathrm{LDH}$ activity are the result of mixtures of the two basic types of LDH. A new difference in the properties of the two enzymes will be presented. LDH extracted from heart, brain, kidney, and lung of rabbits is inhibited noncompetitively by end product (lactic acid), whereas LDH of skeletal muscle and liver is inhibited only by much greater amounts of lactic acid, and the inhibition is competitive. The activity and properties of $\mathrm{LDH}$ in these two groups of tissues with respect to substrate (pyruvate) saturation, substrate inhibition, effect of hydrogen ion concentration, and end-product (lactic acid) inhibition can be correlated with the known susceptibility of the respective tissues to hypoxia, thus providing a possible basis for relative susceptibilities of tissues to infarction. Tissues with the readily inhibited $\mathrm{LDH}$ accumulate sufficient lactic acid to inhibit the use of anaerobic mechanisms for cell survival during hypoxia. Significant differences in both LDH activity and the type of enzyme present were found in studies of comparable infant and adult organs. The changes in enzymic activity and pattern in the maturation of certain organs may in part be related to the replacement of cell types. In other organs, however, such changes occur despite the absence of significant alteration of cell types, suggesting the possibility of environmental control of $\mathrm{LDH}$ activity.

\section{Chronic Unconjugated Hyperbilirubinemia (CUH) with Increased Production of Bile Pigment not Derived from the Hemoglobin of Mature, Circulating Erythro- cytes. IrwiN M. ArIAS, New York, N. Y. (intro- duced by Helen Ranney).}

The role of extramedullary hemolysis in the production of CUH is well known. This study indicates that CUH can result from intramedullary hemolysis without overt signs of extramedullary erythrocyte destruction. A 19 year old white male of Italian descent has had asymptomatic jaundice without hepatosplenomegaly for 6 years. The serum bilirubin ( 1.3 to $4.5 \mathrm{mg}$ per $100 \mathrm{ml}$ ) was unconjugated. Complete blood counts, analysis of hemoglobin including $A_{2}$ and $F$, erythrocyte glucose-6phosphate dehydrogenase and pyruvic kinase activities, liver function tests, and liver histology were normal. The mean reticulocyte count was $1.3 \%$. Bone marrow examinations revealed erythrocyte hyperplasia $(M: E$, $1: 3)$. Erythrocyte survival $\left(\mathrm{Cr}^{51}\right)$ has been normal on four occasions, but fecal urobilinogen excretion has ranged from 580 to $1,110 \mathrm{mg}$ per day (normal 80 to $250 \mathrm{mg}$ per day). In vivo study of glucuronide formation (menthol tolerance test) and in vitro assay of hepatic uridine diphosphoglucose dehydrogenase and glucuronyl transferase activities (using bilirubin, $o$-aminophenol, and 4-methyl umbelliferone as glucuronide receptors) were normal. The transfer of bilirubin from plasma to the liver cells was studied in vitro. Two $g$ of the patient's liver was obtained at surgery. Slices and homogenates were incubated with bilirubin in either 
the patient's or in normal serum. No significant difference was observed in direct-reacting bilirubin formation when compared with similar studies on liver from control subjects. The half-time of disappearance of injected $\mathrm{Fe}^{69}$ from plasma was 20 minutes (normal 90 $\min$ ). Ferrokinetic study revealed a decreased rate of removal of $\mathrm{Fe}^{59}$ from the bone marrow. The patient ingested glycine- $\mathrm{N}^{15}$. The isotope concentration was serially measured in hemin and stercobilin. The average erythrocyte life span was 110 days. At least $49 \%$ of bile pigment was derived from sources other than mature, circulating erythrocytes. Chronic jaundice probably results from increased intramedullary hemolysis. An inherited abnormality may be present, since a younger brother has chronic unconjugated hyperbilirubinemia (1.2 to $3.2 \mathrm{mg} / 100 \mathrm{ml}$ ), normal complete blood counts, a mean reticulocyte count of $1.4 \%$, and erythroid hyperplasia in the bone marrow, and excretes $880 \mathrm{mg}$ of fecal urobilinogen daily.

Metabolism of Levo- and Dextroisomers of Thyroid Hormones in Man. Walter L. Arons, Palo Alto, Calif. (introduced by J. A. Luetscher).

The metabolic fate of $\mathrm{I}^{131}$-labeled $\mathrm{L}$-thyroxine $\left(\mathrm{LT}_{4}\right)$, L-triiodothyronine $\left(\mathrm{LT}_{3}\right)$, D-thyroxine $\left(\mathrm{DT}_{4}\right)$, and $\mathrm{D}$-triiodothyronine $\left(\mathrm{DT}_{3}\right)$ has been compared by administering each orally in representative pharmacologic dosage to 5 or more euthyroid subjects. Serum, urine, and stools have been serially analyzed up to 192 hours for total radioactivity and by paper chromatography for distribution of $\mathrm{I}^{131}$-labeled compounds. The following findings are of note. 1) Both $\mathrm{D}$ isomers are deiodinated as readily as are the naturally occurring, more active $\mathrm{L}$ forms. 2) After $\mathrm{LT}_{4}$ administration, conversion to $\mathrm{T}_{3}$ has been evidenced by the presence of $\mathrm{T}_{3}$ in the urine in several instances. Also after $\mathrm{DT}_{4}, \mathrm{~T}_{3}$ has been repeatedly found in both serum and urine. 3) After $\mathrm{LT}_{4}, \mathrm{DT}_{4}, \mathrm{LT}_{3}$, and $\mathrm{DT}_{3}$ administration, evidence for monoiodotyrosine formation was found in the urine. Studies are currently in progress to define this activity more specifically. Preliminary experiments indicate that the glucuronide conjugates of the administered isomers also are excreted in the urine. 4) About twice as much (78\%) of ingested $\mathrm{DT}_{4}$ activity is excreted in urine and stool in 96 hours as of $\mathrm{LT}_{4}$ activity $(45 \%)$. Similar total amounts of administered $\mathrm{LT}_{3}$ and $\mathrm{DT}_{3}$ activity are excreted in 96 hours $(80 \%)$. However, significantly more $\mathrm{DT}_{4}$ and $\mathrm{DT}_{3}$ than the $\mathrm{L}$ forms are excreted in the stools. These data, in combination with the serum activity measurements, indicate a probably poorer gastrointestinal absorption of the $D$ isomers. 5) External body counting has indicated that all four isomers, but $\mathrm{LT}_{4}$ and $\mathrm{DT}_{4}$ in particular, are concentrated in the liver. These data reveal both significant differences and similarities in the metabolic fate of the $L$ and $D$ isomers of thyroid hormones and provide some basis for interpreting the differing pharmacologic activity of these substances in man.
The Mechanism of Steroid Granulocytosis. Jон N W. Athens,* Otto P. HaAb, Spencer O. Raab, Dane R. Boggs, George E. Cartwright* and Maxwell M. Wintrobe,* Salt Lake City, Utah.

The purpose of this study was to determine the mechanism by which adrenocorticosteroids produce granulocytosis. To determine whether the granulocytosis is due to a shift of cells from the marginal granulocyte pool (MGP) to the circulating granulocyte pool (CGP), the size of these compartments and of the total blood granulocyte pool (TBGP) was measured in ten subjects. The TBGP increased from 48 to $89 \times 10^{7}$ cells per $\mathrm{kg}$ after 10 days of oral prednisone $(40 \mathrm{mg} /$ day). The TBGP increased from 79 to $138 \times 10^{7}$ cells per $\mathrm{kg}$ 5 hours after the intravenous injection of $200 \mathrm{mg}$ of hydrocortisol. In both instances it was found that the granulocytosis was due to an increase in size of both the MGP and CGP. To assess whether the granulocytosis is due to an increased marrow output of cells, the effect of the rapid injection of hydrocortisol on the specific activity of granulocytes in the circulation was evaluated in five subjects. If the granulocytosis were due to an increased influx of unlabeled cells from the marrow, a sudden decrease in specific activity of the blood granulocytes should have occurred. No such decrease was observed. To assess whether steroids impair the egress of cells from the circulation, the cellularity of inflammations produced in the skin was measured before and after steroids. The cellularity of these exudates was reduced $75 \%$ by steroids. From these studies it is concluded that steroids produce granulocytosis by decreasing the egress of cells from the circulation and not by causing an influx of cells from the marrow or by producing an intravascular shift of cells. These studies may have significance in regard to the propensity of adrenocorticosteroids to increase susceptibility to infection.

Adrenomedullary Effects of Guanethidine. William J. Athos, Edwin Fineberg, Burton McHugh and James G. Hilton,* New York, N. Y.

Guanethidine effect on adrenal catecholamine secretion was studied: a) by perfusion into the arterial circuit of isolated denervated adrenal gland preparations of Hilton et al. (Amer. J. Physiol. 1958, 192, 525), and $b$ ) by single intravenous injection into intact dogs with cannulated adrenal veins. Control mean secretory rates of norepinephrine and epinephrine from isolated denervated preparations were 0.041 and $0.070 \mu \mathrm{g}$ per minute, respectively. During guanethidine perfusion at $10 \mu \mathrm{g}$ per minute, there was no change in catecholamine secretion. Glucagon perfusion at the end of each experiment showed a rise in norepinephrine and epinephrine secretion to mean rates of 0.37 to $0.61 \mu \mathrm{g}$ per minute, respectively, indicating viability of the glands. Histamine perfusion $(0.36 \mathrm{mg} / \mathrm{min})$ studied in a similar way was also without effect on medullary secretion. 
In the intact dogs with innervated adrenals, single intravenous guanethidine injections in dosages from 1.0 to $10.0 \mathrm{mg}$ per $\mathrm{kg}$ induced an initial mean peripheral blood pressure drop, followed in 2 to 3 minutes by a prominent pressure elevation lasting 20 to 60 minutes. This was associated with a significant fall in adrenal epinephrine secretion to $50 \%$ of control. Norepinephrine secretion remained unchanged from control. Intravenous histamine $(0.1$ to $0.25 \mathrm{mg} / \mathrm{kg})$ in similar intact preparations resulted in abrupt drops in mean peripheral blood pressure associated with a 20 - to 40 -fold rise in epinephrine and a 3 -fold rise in norepinephrine secretion.

Induction of Acute Iodine Depletion and the Response thereto in Normal Subjects and in Patients with Graves' Disease. Russel M. Barakat and Sidney H. INGBAR, * Boston, Mass.

To ascertain whether self-regulatory mechanisms exist within the human thyroid, a means of inducing acute iodide deficiency was sought. Nine patients, including two with thyrotoxicosis, were fed low iodine, anionexchange resin-treated milk (chloride cycle) for 2 days. On the second day, brisk osmotic diuresis was induced by infusion of mannitol. Cations were replaced as iodide-free chloride salts. Iodide kinetics were assessed with $\mathrm{I}^{131}$ during a control period, during the osmotic diuresis, and 14 to 24 hours thereafter. Renal iodide clearances increased during mannitol infusion. Acute iodide depletion was induced since, after diuresis, plasma concentration and total body content of inorganic $\mathrm{I}^{127}$, calculated from the specific activity of urinary iodide, averaged $50 \%$ less than control values. Concomitantly, thyroid iodide clearance rates and 3-hour $\mathrm{I}^{131}$ accumulation increased by an average of $100 \%$. Although $2 \mathrm{mg}$ of iodide, when acutely administered, does not normally reduce thyroid $\mathrm{I}^{131}$ accumulation, inclusion of $800 \mu \mathrm{g}$ of iodide in mannitol infusions prevented the subsequent decline in plasma iodide concentration and the increase in thyroid clearance rates. This suggested that iodide depletion per se was the cause of increased thyroid function. This response could not be ascribed to increased secretion of endogenous thyrotropin. It was demonstrable within 4 hours after initiation of diuresis, sooner than the stimulation of iodine accumulation induced by exogenous thyroid-stimulating hormones (TSH). Furthermore, plasma protein-bound iodines neither decreased during diuresis nor increased subsequently. Finally, separate experiments revealed no activation of the TSH-dependent, iodide-trapping mechanism when hormonal synthesis was completely blocked by methimazole for several days. These findings suggest that there exists within the thyroid an intrinsic, extrahypophyseal mechanism for increasing function, which is responsive to depletion of plasma, and hence intrathyroidal, iodide and which may be related to the pathogenesis of diseases characterized by functional autonomy of the thyroid gland.
Characteristics and Performance of the Left Ventricle in Massive Mitral Insufficiency. F. A. BASHOUR AND J. D. Khalaf, Dallas, Tex. (introduced by Carleton B. Chapman).

Left ventricular volumes (end-diastolic and end-systolic) were measured in 8 patients with massive mitral insufficiency, using the biplane cineangiofluorographic method of Chapman and Baker and a modification of Arvidsson's technique. In 3 patients a minimal degree of aortic insufficiency was demonstrated by retrograde aortography. The end-diastolic volume (EDV) ranged from 116 to $271 \mathrm{ml}$ as compared with the normal range of 85 to $128 \mathrm{ml}$. The end-systolic volume (ESV) ranged from 32 to $76 \mathrm{ml}$ as compared with the normal range of 35 to $43 \mathrm{ml}$. The absolute stroke volume and stroke volume index averaged $144 \mathrm{ml}$ and $87.8 \mathrm{ml}$ per $\mathrm{m}^{2}$, respectively. A linear relationship was observed between the stroke volume and EDV. The efficiency of the left ventricular ejection (ratio of ESV to EDV) ranged from 20 to $36 \%$ and averaged $28 \%$, as compared with the normal ratio of $39 \%$. The mean rate of ventricular ejection was calculated in 7 patients. It ranged from 182 to $860 \mathrm{ml}$ per second and averaged 475, as compared with the values obtained in 2 normal subjects ( 264 and $272 \mathrm{ml} / \mathrm{sec}$ ). The tension-time index ranged from 2,325 to $3,415 \mathrm{~mm} \mathrm{Hg}$-sec per minute and averaged 2,930 as compared with the values obtained in 2 normals $(1,940$ and $2,390 \mathrm{~mm} \mathrm{Hg}-\mathrm{sec} / \mathrm{min})$. The mean thickness of the left ventricular wall during diastole was slightly higher than in the normals, measuring 10 and $7 \mathrm{~mm}$, respectively. These results demonstrate: 1) the existence of increased left ventricular volumes and muscle mass in mitral insufficiency; 2) the adequacy of the left ventricular ejection; and 3) a rise in the tension-time index, a determinant of myocardial oxygen consumption.

\section{Analog Computer Extrapolation of Indicator Dilution Curves. E. J. Battersby and Elliot V. Newman,* Nashville, Tenn.}

In the presence of left-to-right shunts, the measurement of pulmonary blood flow by indicator injection into the right heart with peripheral arterial sampling is usually precluded by the early recirculation of indicator. This masks all but the initial portion of the primary circulation curve. Theoretically, the ideal method for recovering the masked portion of the curve would involve a function, mathematical or analog, that would accurately describe the whole curve. When matched to the available portion of the primary curve it would predict the remainder. Such a function can be produced by an electronic analog computer: $y(x)$ when $d y / d x=[G(x)$ $-y] / K ; G(x)$ is a normal distribution function and $K$ is the time constant of a unit lag component. Visual match by repeated solution to whole primary curves in man and dog has proved satisfactory. The ability of this analog to predict whole primary curves from 
anterior segments was tested in dogs with brachiocephalic artery-to-right atrium shunts. Indicator dye injections were made into the right atrium and sampled in the left subclavian artery for computer curves. Control curves between the right atrium and pulmonary artery or left atrium and subclavian artery had their areas calculated in the usual manner. The anterior portions of computer curves were matched and extrapolated. Thirty-four computer-extrapolated curve areas expressed as percentage of control curve area gave values of $103.1 \pm 11.7$ ( $1 \mathrm{SD}$ ). Forward triangle analysis of the same curves yielded values of $94.7 \pm 17.0$ $(K=0.329)$. These data indicate that the computer extrapolation method is superior to methods employing empiric constants whose value changes with flow, volume, injection site, and species.

Adult Celiac Disease: Rapid Sequential Changes in Jejunal Mucosa with Alterations of Dietary Gluten. Theodore M. Bayless, John H. Yardley, James H. Norton and Thomas R. Hendrix, Baltimore. Md. (introduced by Ivan L. Bennett, Jr.).

Objective clinical improvement in adult celiac disease (nontropical sprue) with a gluten-free diet is well documented. On the other hand, reversibility of the jejunal lesion has been questioned. Peroral intestinal biopsy during periods with and without gluten could give important information about the nature of this disorder. Eight patients with severe jejunal alterations were studied. After gluten exclusion, improvement in mucosal histology was seen in all. The disordered surface epithelium became tall and well arranged. Recognizable villus structures appeared in the five patients on treatment for 3 or more months. Biopsies in one patient after 2 years and in another after 3.5 years were virtually normal. In order to determine the rapidity of this epithelial improvement, biopsies were done in two patients 3,7 , and 10 days after gluten exclusion. The pretreatment mean cell height of the surface epithelium was $21 \mu$. After 7 days, mean height had increased to normal $(32 \mu)$. One of the patients with a virtually normal jejunal biopsy was given wheat gluten for 6 days. Daily fecal fat excretion of $2.4 \mathrm{~g}$ became abnormal on the first day $(8.7 \mathrm{~g})$ and then rose stepwise to $17 \mathrm{~g}$ on the seventh. Biopsies were done after 1, 3, and 6 days. Focal epithelial degeneration at villus tips with edema and congestion of the lamina propria was seen after 1 day and became progressively more widespread and severe until gluten was withdrawn. By 6 days the mean height of the surface epithelium had decreased to the untreated celiac range and the number of mitotic figures in the crypts had trebled. Gluten was in some way toxic to the exposed epithelial cells. These observations strongly suggest that the epithelial lesion seen in celiac disease is indeed gluten-induced and is rapidly reversible on gluten exclusion.
The "Induction" of Dihydrofolic Reductase Activity in Erythrocytes and Leukocytes of Subjects Treated with Amethopterin. J. R. Bertino, M. E. Fink AND P. Calabresi, New Haven, Conn. (introduced by D. Seligson).

Tetrahydrofolic acid, formed from folic or dihydrofolic acid in a reaction catalyzed by the enzyme, dihydrofolic reductase, plays an important role in the biosynthesis of purines and thymidylic acid. This enzyme combines avidly with amethopterin and is inhibited by low concentrations $\left(10^{-8} \mathrm{M}\right)$ of the antimetabolite. After treatment with amethopterin, increased enzyme activity has been detected in leukemic leukocytes and also in normal circulating leukocytes and erythrocytes. The mechanism of this adaptive enzyme formation has been investigated in the peripheral blood cells of 14 subjects after a single 20 -mg dose of amethopterin, administered intravenously. Dihydrofolic reductase activity appeared in the erythrocytes in 3 to 4 days, reached peak levels at 8 to 9 days, and was measurable for as long as 4 months. In normal leukocytes, enzyme activity appeared in 3 to 4 days, reached peak levels at 9 to 11 days, and could not be detected after 19 to 25 days. When lysates of leukocytes were assayed for ability to inhibit dihyrofolic reductase, the concentration of the amethopterinlike inhibitor paralleled the increased levels of enzyme activity observed in the leukocytes. Agents that affect granulocyte production, such as vinblastine and busulfan, suppressed the magnitude of induced enzyme activity. Folinic acid, a competitive inhibitor of dihydrofolic reductase in vitro, also depressed this response in vivo. These findings suggest that: 1) dihydrofolic reductase activity may be under feedback control of reduced folic acid compounds; 2) its appearance in circulating leukocytes is inhibited by myelosuppressive agents; and 3 ) once induced, the enzyme activity appears to parallel the estimated life span of the cell and may provide another method for measuring leukocyte and erythrocyte survival.

The Effect of Ethanol-induced Fatty Liver on the Transport of Triglyceride Fatty Acids from Liver to Plasma. A. Bezman, P. J. Nestel, J. M. Felts, R. J. Havel * AND S. French, San Francisco, Calif.

Circulating free fatty acids esterified in the liver are a precursor of triglyceride fatty acids (TGFA) contained in plasma very low density $(<1.006)$ lipoproteins (VLDLP). Isotopic studies in rabbits have shown that TGFA contained in the subcellular fractions of normal liver and TGFA of blood plasma equilibrate rapidly. Two human subjects were studied when they had acute fatty liver due to alcohol and later when the hepatic triglyceride content had decreased. Palmitate-1-C $C^{14}$ was injected intravenously. Serial blood samples were taken and a sample of liver obtained 2 to 3 hours later. Specific activities of those TGFA contained in VLDLP and of hepatic TGFA were measured. In all experiments: 1) the peak specific activities of 
plasma TGFA occurred 2 to 3 hours after injection; 2) the ratio of the peak specific activity in plasma TGFA to that of the liver was inversely related to the concentration of hepatic TGFA. Similar studies were performed in two rabbits fed ethanol for prolonged periods of time. In these experiments small pieces of liver were taken at intervals and separated into floating fat, mitochondria, microsomes, and supernate. Plasma TFGA equilibrated rapidly with TGFA of microsomes and mitochondria; however, the floating fat of these livers, which contained most of the hepatic TGFA, had much lower specific activity than other subcellular liver fractions and plasma TGFA. Thus, in ethanol-induced fatty liver, TGFA of the floating fat only very slowly equilibrated with the other subcellular fractions of liver. Nevertheless, the time of peak specific activities of the TGFA of VLDLP was found to be within normal limits.

The Heterogeneity of Particulate Fat in Plasma. Edwin L. Bierman, Enoch Gordis and James T. Hamlin, III, New York, N. Y. (introduced by Vincent P. Dole).

Two distinct groups of fat particles in turbid plasma can be identified by zone electrophoresis (starch granules) and by differential flocculation. One fraction migrates with $\alpha_{2}$-globulin, forms a dense flocculate at the top of a polyvinylpyrollidone (PVP) gradient (concentration range 0 to $5 \%$ ), and appears to be identical with lymph chylomicrons. These particles are relatively large (diameter $>200 \mathrm{~m} \mu$ ). The other fraction (of smaller average size) migrates with $\beta$-globulin, flocculates at the bottom of the PVP gradient, and is believed to come from sources other than thoracic duct lymph. Both kinds of particle are low enough in density to float in saline. Chromatographic analyses of the two groups of particles in plasma taken during alimentary lipemia have shown that the fatty acid composition of triglycerides in " $\alpha_{2}$-particles" resembles that of the feeding, although not identically. This fraction appears early and reaches a maximum 4 to 6 hours after the feeding. The " $\beta$-particles" increase in number during this period, become the predominant form by 9 hours, and are the last to be detected at the end of absorption. Their fatty acid composition, initially closer to that of body fat, shifts toward the composition of the fed fat as absorption proceeds. Changes in the particulate lipid zones on starch block electrophoresis are paralleled by corresponding changes in the turbid zones distinguishable on the PVP gradient. All the fat particles in human thoracic duct lymph, labeled by a previous feeding of $\mathrm{C}^{14}$-plamitic acid, migrate in the $\alpha_{2}$-globulin region. When this material is injected intravenously, label soon appears in the $\beta$-particles. The findings are consistent with the hypothesis that two kinds of fat particles are formed during alimentary lipemia: the chylomicrons, produced by the intestine, and a second group containing a mixture of triglycerides derived from chylomicrons and pre-existing fat stores.
Altered Autonomic Nervous System and Renal Responses in Primary Aldosteronism. EDWARD G. BIGLIERI, Malcolm B. McIlroy,* Arnold Naimark and Peter H. Forsham,* San Francisco, Calif.

Patients with autonomic insufficiency and five subjects with primary aldosteronism had a prompt and sustained diuresis and natriuresis associated with a rise in glomerular filtration rate during infusion of isotonic saline at a rate of $50 \mathrm{ml}$ per minute for 60 minutes. This similarity in response led to the investigation of baroreceptor reflex activity in the latter group in view of the known impairment of this mechanism in the former. Patients with primary aldosteronism had a similar impairment of circulatory reflex control: a lack of hypertensive overshoot and bradycardia following Valsalva's maneuver, and a fall in blood pressure and pulse pressure without change in pulse rate when assuming an upright posture. These responses became normal as early as 2 weeks after surgical removal of unilateral adenomata. Normo- or hypokalemic patients with essential hypertension and normal subjects given large doses of salt-retaining hormone for 2 to 4 weeks responded normally to salt infusions and revealed physiologic circulatory reflex responses. This suggests that profound electrolyte abnormalities and excess of aldosterone must coexist to produce the observed alterations in function of the renal and automatic nervous systems.

Measurements of Leukocyte Intracellular $p H$ in Chronic Myelocytic Leukemia. Jerome B. Block and David P. Rall, Bethesda, Md. (introduced by Wallace $P$. Rowe).

Intracellular $\mathrm{pH}\left(\mathrm{pH}_{1}\right)$ measurements may be useful in characterizing cell populations. Viable leukocyte preparations obtained from patients with chronic myelocytic leukemia by techniques of plasmapheresis and dextran sedimentation were suspended in Krebs III buffer with added glucose at $37^{\circ} \mathrm{C}$. In 70 experiments at varied buffer $\mathrm{pH}\left(\mathrm{pH}_{\mathrm{e}}\right), \mathrm{pH}_{1}$ was determined with the weak acid $\mathrm{C}^{14}$-labeled 5,5-dimethyl 2,4-oxazolidinedione (DMO) or the weak base $\mathrm{C}^{14}$-labeled Tris by measuring the concentration of $\mathrm{DMO}$ or Tris in the intracellular water and extracellular buffer. $\mathrm{pH}_{1}$ was found to be dependent on $\mathrm{pH}_{e}$. With $\mathrm{DMO}$, below $\mathrm{pH}_{e} 7.4, \mathrm{pH}_{t}$ was maintained higher than $\mathrm{pH}_{\mathrm{e}}$ by approximately 0.5 to 1.0 $\mathrm{U}$; above 7.4 this gradient was reversed. The apparent $\mathrm{pH}$ gradient was not altered by incubation at $5^{\circ} \mathrm{C}$ or increasing cell acid production with various drugs, nor was it related to the clinical hematologic status. However, $n$-butanol, arsenate, or other cell injury resulted in reduction of the gradient. Determinations using Tris resulted in uniformly lower values of $\mathrm{pH}_{1}$ as compared with the use of DMO. No cellular or protein binding of Tris was found to account for this discrepancy. These observations suggest that a $\mathrm{pH}$ gradient may be maintained across the leukocyte membrane. If $\mathrm{pH}_{4}$ is homogeneous throughout the cell, estimates of $\mathrm{pH}_{1}$ based on nonionic diffusion should be similar with both DMO 
and Tris. The dissimilar results obtained suggest intracellular nonhomogeneity with respect to $\mathrm{pH}$.

Hypersensitivity to Staphylococcal Filtrates Producing Fever in Rabbits. Phyllis Bodel and Elisha Atkins,* New Haven, Conn.

Although many toxins have been isolated from Staphylococcus aureus, their role in the pathogenesis and manifestations of staphylococcal disease remains uncertain. When broth filtrates of staphylococci were injected into rabbits, fever was produced only by those from certain strains. Rabbits initially unresponsive to filtrate from 80-81 staphylococci acquired a febrile response after intravenous or intradermal infection with the homologous organism. Other responses to intravenous inoculation of filtrate, including lymphopenia and rapidly developing tolerance to repeated injections, are similar to those seen in systems involving delayed hypersensitivity. Moreover, febrile response to $80-81$ filtrate could be passively transferred to unreactive normal recipients with mononuclear cells from previously infected donor animals. Serum transfer was not effective. Filtrates of VA, and 80-81 strains were studied to isolate the pyrogenic fraction. By methods similar to those used to obtain purified protein derivative, a heat-stable, antigenic protein fraction was recovered which contained the pyrogenic activity. Carbohydrate fractions were inactive. The pyrogen did not resemble endotoxin. The relationship of filterable pyrogen to fever-inducing substances obtained from both whole and disrupted staphylococcal cells as well as to known staphylococcal toxins such as $\alpha$-hemolysin and coagulase will be discussed. These studies indicate that delayed hypersensitivity to staphylococcal products, whether occurring naturally owing to unapparent infection or induced by infection in the laboratory, produces fever in rabbits. They also suggest that such hypersensitivity may play a significant role in the manifestations of staphylococcal disease. The role of the staphylococcal toxins may acquire new significance in view of the close resemblance of staphylococcal disease to such chronic diseases as tuberculosis and systemic fungus infections where hypersensitivity has been clearly implicated.

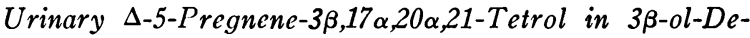
hydrogenase Deficiency. Alfred M. Bongrovanni* and Albert Clark, Philadelphia, $\mathrm{Pa}$. and Montreal, Canada.

To date 7 cases of the adrenogenital syndrome due to $3 \beta$-ol-dehydrogenase deficiency have been studied. As reported previously the major urinary steroids in all were present, not in the fraction hydrolyzed by $\beta$-glucoronidase but in the residue, after solvolysis and digitonin precipitation. A novel compound, $\Delta-5$-pregnene- $3 \beta, 17 \alpha$,$20 \alpha, 21$-tetrol, has been isolated in this condition. The identity is based on chemical characterization, elemental analysis, synthesis of the proposed compound, and suitable comparisons between the synthetic and natural substances.
This finding is of interest in that it indicates the 21hydroxylation of steroids with the $\Delta-5$-pregnene- $3 \beta$-ol configuration, a circumstance hitherto considered unlikely under natural conditions. Contrary to our earlier report, not all of these subjects are salt losers nor is the condition uniformly fatal. A partial block with compensatory cortisol biosynthesis is evident in the milder form. In the two males studied one had cryptorchidism and the other was a male pseudohermaphrodite with anatomical structures of the female. Despite the ambiguity of the external genitals in the females, due to labial fusion, careful radiologic study reveals discrete urethral and vaginal orifices rather than a common urogenital sinus. The clinical implications of this will be discussed. Finally, one of the cases also excreted significant quantities of $3 \alpha, 17 \alpha, 20 \alpha$-pregnanetriol, suggesting a double block including 21-hydroxylase. In this last case the novel compound herein described was not present.

An Abnormal Pathway of Steroid Metabolism in Patients with Glucose-6-Phosphate Dehydrogenase Deficiency. Abraham J. Borkowski, Paul A. Marks,* Fred H. Katz, Marvin M. Lipman and Nicholas $P$. Christy,* New York, N. Y.

Before hepatic conjugation with glucuronic acid, steroid hormones are reduced in the A-ring. TPNH is essential for this reduction. In subjects deficient in hepatic glucose-6-phosphate dehydrogenase (G6PD), the ensuing relative insufficiency of TPNH might be supposed to result in a decreased capacity to reduce the steroidal A-ring, with a consequent increase in excretion of compounds not so reduced. This hypothesis was tested. In five patients with G6PD deficiency, resting excretion of a nonreduced metabolite of cortisol, $6 \beta$ hydroxycortisol $(6 \beta-\mathrm{OH}-\mathrm{F})$ was studied. The steroid was extracted and chromatographed by the method of A. G. Frantz; after isolation, eluates were quantified against authentic standard by the Porter-Silber reaction. Losses were corrected for by measuring the loss of radioactivity of added $4-\mathrm{C}^{14}-6 \beta-\mathrm{OH}-\mathrm{F}$ (recovery, 25 to $50 \%$ ). Mean daily excretion of $6 \beta-\mathrm{OH}-\mathrm{F}$ by the 5 patients was considerably higher than normal (751 $\mu \mathrm{g}$, range 487 to 1,$147 ; 16$ normal subjects, mean 349 , range 156 to $555 ; \mathrm{p}<0.01$ ), and significantly higher than in nine patients with hepatic disease (mean 340 $\mu \mathrm{g}$ ). In six subjects $100 \mathrm{mg}$ cortisol was rapidly infused intravenously to tax the A-ring reducing mechanism acutely. In three, an excessive amount of administered steroid was metabolized to $6 \beta-\mathrm{OH}-\mathrm{F}(19.6,10.1$, and $6.0 \mathrm{mg} / 24$ hours; normal not $>3.5 \mathrm{mg}$ ); and in three, a subnormal quantity of exogenous steroid was excreted as two major A-ring reduced cortisol metabolites, tetrahydrocortisol (THF) and tetrahydrocortisone (THE) (patients, 11 to $22 \mathrm{mg}$; normal 26 to $30 \mathrm{mg}$ / 24 hours). Plasma half-time of infused cortisol was normal. Glucuroconjugation was normal in rate and amount as measured by normal metabolism of a reduced steroid (75 mg THE, intravenously). The data show increased excretion of cortisol in the form of a com- 
pound not reduced in the A-ring. Although other hypotheses are possible, utilization of this abnormal pathway can be explained by insufficiency of TPNH generation owing to hepatic deficiency of G6PD.

Electrophoretic Heterogeneity of Glucose-6-Phosphate Dehydrogenase and Its Relationship to Enzyme Deficiency. Samuel H. Boyer and IAN H. Porter, Baltimore, Md. (introduced by Victor A. McKusick).

The function of the $\mathrm{X}$-linked genetic locus governing glucose-6-phosphate dehydrogenase (G6PD) deficiency is unknown. It is uncertain whether this locus governs structure or is concerned with regulation of enzyme activity through some unknown nonstructural mechanism. The detection of qualitative variation might suggest, on the model of the hemoglobinopathies, that the X-linked locus specifies enzyme structure. We have detected qualitative variation of G6PD after starch gel electrophoresis of crude hemolysates from Negro subjects. Such variation was not detected in Caucasians. By this method two G6PD types, $\mathrm{A}$ and $\mathrm{B}$, were distinguishable in males; $0.169(40 / 236)$ were $A, 0.648$ (153/236) were $B$, while $0.182(43 / 236)$ were G6PD deficient and not directly typable. Distinction between $A$ and $B$ types is also evident after electrophoresis of purified enzyme preparations. Crude hemolysates proved unsatisfactory for typing female subjects. Examination of leukocyte homogenates indicates that three G6PD types, $A, A-B$, and B, are evident in Negro females. The A type is less common in females than it is in males. A relationship between G6PD deficiency exists, in that all examined Negro males with G6PD deficiency have leukocyte G6PD which is type A. No differences in $\mathrm{pH}$ optimum, $K_{m}-G 6 P$, heat stability, Arrhenius constant or inactivation by dehydroisoandrosterone were detected on comparison of purified preparations of A and B. Statistical, genetic, and electrophoretic evidence suggests that the locus governing the G6PD $A$ and $B$ polymorphism is $\mathrm{X}$-linked and, moreover, appears to be the same locus as that governing G6PD deficiency. Three alleles are postulated: A normal, B normal, and A deficient. The existence of electrophoretic variation suggests that the $\mathrm{X}$-linked locus has a structural function and, in turn, that G6PD deficiency is produced by a structural alteration of the enzyme.

Renal Hemodynamic Effects of Mannitol Infusion. WILliam E. Braun and Lawrence S. Lilienfield, Washington, D. C. (introduced by Edward D. Freis).

The clinical success reported with mannitol infusions has prompted a re-evaluation of its renal hemodynamic effects. Anesthetized dogs were studied after laparotomy and insertion of ureteral and renal venous cannulae. Clearance and extraction of para-aminohippurate (PAH) and clearance of inulin (GFR) were measured during control periods, during periods of $20 \%$ mannitol infusion and during periods of hemorrhagic hypotension [mean arterial pressure $(\mathrm{MAP})=75 \mathrm{~mm} \mathrm{Hg}$ ] with and without mannitol infusions. Renal plasma flow (RPF) was calculated from PAH clearance and extraction values. During mannitol infusions in normotensive dogs, the extraction of PAH consistently and significantly decreased while the clearance of $\mathrm{PAH}$ remained relatively unchanged. Inulin clearance consistently decreased. Decrease in extraction indicates change in blood flow distribution in the kidney; i.e., more blood perfusing nonfunctioning areas such as the medulla. Decreased GFR in the face of increasing RPF is explained by dilatation of efferent arterioles. In the anesthetized dogs made hypotensive by hemorrhage, PAH clearance, inulin clearance, and calculated RPF fell markedly. When $20 \%$ mannitol was then constantly infused into these dogs RPF rose toward control values without significant changes in MAP. In dogs receiving a constant infusion of $20 \%$ mannitol during the induction of hemorrhagic hypotension, PAH clearances and GFR fell to a lesser extent than in those with hemorrhagic hypotension alone. In all cases during the infusion there was a significant reduction in PAH extraction. Thus, $\mathrm{RPF}$ was restored to, or remained near control values. These data support the hypothesis that a $20 \%$ mannitol infusion dilates constricted efferent arterioles, especially those of the juxtamedullary glomeruli, perhaps by interrupting or abolishing renal sympathetic vasoconstrictor reflexes.

Determinants of Ventricular Dimensions in Intact Unanesthetized Man. Eugene Braunwald,* Allan Goldblatt and Donald C. Harrison, Bethesda, Md.

Although ventricular volume has been considered an important variable in circulatory regulation, little information is available on the determinants of ventricular size in man. Accordingly, a method was developed for the precise measurement of ventricular dimensions throughout the cardiac cycle in closed-chest, intact, unanesthetized human subjects. Three silver clips were sutured to the surface of either or both ventricles of 25 patients undergoing cardiac surgery and, after recovery, cineradiograms were obtained at 30 frames per second. The distance between the clips on the individual frames was measured and related to the intraventricular pressures, which in four subjects were recorded simultaneously through a catheter with a micromanometer mounted at its tip. During systole, ventricular dimensions decreased by an average of $18.5 \%$ of the enddiastolic dimensions. In all six patients studied during normal inspiration, an increase in right ventricular enddiastolic dimensions, averaging $3.2 \%$, occurred, but there were no detectable or consistent changes in left ventricular dimensions. During deep inspiration, right ventricular end-diastolic dimensions increased by an average of $9 \%$ of those existing during expiration; the magnitude of the systolic decrease always varied directly with the end-diastolic size of the preceding cycle, an observation supporting the concept that Starling's law of the heart operates in intact man. In five patients isoproterenol consistently decreased end-systolic size by values up to 
$13 \%$ of control, increased the rate at which the clips approached each other during systole, but did not consistently alter end-diastolic dimensions. In six patients, pedaling a bicycle ergometer in the supine position consistently decreased both end-diastolic and end-systolic dimensions by an average of 5.4 and $5.0 \%$ of control values. In conclusion, the effects on ventricular dimensions of a variety of basic interventions have been elucidated in intact man.

Genetic Implications of Diisopropyl Fluorophosphate $(D F P)^{32}$-Erythrocyte Survival Studies in Negro Females Heterozygous for Glucose-6-Phosphate Dehydrogenase Deficiency. George J. Brewer, Alvin R. Tarlov and Robin D. Powell, Chicago, Ill. (introduced by Alf S. Alving).

Erythrocyte survival curves ( $\mathrm{DFP}^{32}$-labeling method) were obtained twice in each of 14 subjects, once 4 months before and once during administration of $30 \mathrm{mg}$ primaquine daily for 84 days. Subjects were five normal Caucasian females, two normal Negro males, one Negro male and four Negro females with fully expressed G6PD deficiency, and two heterozygous females with intermediate G6PD deficiency (G6PD values 65\% and $30 \%$ of mean normal, respectively). Drug administration to enzyme-deficient subjects (both full and intermediate expression) produced curves having two linear phases-an initial brisk fall, then a less rapid fall. From the latter, the life span of those erythrocytes not initially destroyed by drug was determined during continued drug administration and compared with predrug values. Results: 1.) In normal female subjects, primaquine caused significant shortening of the erythrocyte life span (predrug average: 114 days; with drug, 96 days). 2.) In fully expressed G6PD-deficient subjects receiving drug, approximately $50 \%$ of the erythrocytes were destroyed initially; the life span of the remaining cells was $63 \pm 7$ days. 3.) In the two heterozygotes receiving drug, initial red cell destruction was 42 and $72 \%$; the life span of the remaining cells was 66 and 75 days, respectively; there was no segment of the erythrocyte population whose survival was comparable with that of normal controls. Results in the two heterozygotes indicate that the "mosaic" hypothesis of X-chromosomal inactivation, which suggests two genetically different populations of cells in heterozygotes, is not applicable in G6PD deficiency. This hypothesis, recently extended to include G6PD deficiency, suggests a variable mixture of populations of completely normal and mutant erythrocytes in heterozygotes. The studies reported here demonstrate that all erythrocytes in the heterozygous G6PD-deficient subjects are involved with the genetic defect.

Active "Dozenhill" Sodium Transport Across the Isolated Frog Skin. NeAL S. BRICKer* AND Thomas Biber, Copenhagen, Denmark.

Frog skin epithelial cells represent a prototype of systems which actively transport sodium transcellularly while simultaneously preserving their own intracellular fluid composition. These cells exhibit striking functional polarization: at the external surface, $\mathrm{Na}$ enters cell water passively; at the internal surface $\mathrm{Na}$ is actively extruded and $\mathrm{K}$ moves bidirectionally-inward by active transport, outward by passive diffusion. Active transport of $\mathrm{Na}$ and $\mathrm{K}$ often is attributed to a single pump with $1: 1$ linkage. The pump therefore would be nonelectrogenic, and transcellular potential difference (PD) would relate mainly to $\mathrm{Na}$ and $\mathrm{K}$ diffusion potentials. In the steady state, $\mathrm{Na}_{\mathbf{P}}$ influx $=\mathrm{Na}_{\mathbf{A}}$ efflux $=\mathrm{K}_{\mathbf{A}}$ influx $=\mathrm{K}_{\mathbf{P}}$ efflux ( $P=$ passive; $A=$ active). In the present studies, $K_{P}$ efflux was modified by exposing the internal surface of the isolated frog skin to potassium concentrations greater than estimated intracellular concentrations. Frog Ringer's was used as the external solution. PD short circuit current (SCC) and electrical resistance all decreased initially. PD stabilized at approximately $20 \%$ of control values, resistance remained low, but SCC rose, frequently exceeding control values. Net sodium transport not only persisted, but appreciably exceeded SCC. This unusual discrepancy between these two parameters was related largely to transcellular movement of $\mathrm{K}$. Because $\mathrm{Na}$ transport occurred down a steep electrochemical gradient, the process could be entirely passive. However, during complete short circuiting, the ratio of unidirectional sodium fluxes (using $\mathrm{Na}^{22}$ and $\mathrm{Na}^{24}$ ) appreciably exceeded the ratio of sodium concentrations. Furthermore, after addition of strophanthin $(1.4 \times$ $10^{-4} \mathrm{M}$ ), flux ratios promptly fell to equal concentration ratios. Finally, when the $\mathrm{Na}$ gradient was abolished by using hyperosmotic internal solutions ( $\mathrm{Na} 114, \mathrm{~K} 120$ mmoles/L), net $\mathrm{Na}$ transport continued. These data therefore appear to document the phenomenon of downhill active sodium transport.

Water-Water Interaction during Solvent Flow in Isolated Turtle Bladders. William A. Brodsky* AND Theodore P. Schilb, Louisville Ky.

Isolated turtle bladders, filled with hypotonic solutions of sucrose, and immersed in oxygenated Ringer's solutions, were impermeable to serosal electrolyte and to mucosal sucrose. Consequently, a fairly pure process of osmosis was observed. Osmotic flow of solvent (relative weight loss/hour of bladder sac and content) was proportional to the square of the transmembrane osmotic gradient, or $R=R w(\Delta O s)^{2} /(\Delta O s)_{w}{ }^{2}$, where $R$ is rate of weight loss of hemibladder with sucrose divided by the rate of its paired control containing $110 \mathrm{mM} \mathrm{NaCl}$; $R w$ is rate of weight loss of a hemibladder with distilled water divided by the rate of its paired control; $\Delta O s$ is serosal minus sac fluid osmotic activity in sacs with hypotonic sucrose; and $(\Delta O s)_{w}$ is serosal minus sac osmotic activity for sacs containing distilled water. The approximate equality between theoretically derived and experimentally obtained values of net relative water flow confirms the concept that osmotic flow of solvent is due to a two-force mechanism. One force, the chemical potential gradient, initiates difflusional flow of water; 
the other force, frictional drag of flowing water upon surrounding water, accelerates flow of solvent. Addition of salt to mucosal fluid adds a third force-that due to active ion transport. Water movement occurs across sacs containing $\mathrm{NaCl}$ even though transmembrane $\Delta O s$ $\leq 0$, but does not occur across sacs containing isoosmotic sucrose alone.

Human Erythrocyte $p H$ with Special Reference to Intracellular Acidosis in Hereditary Spherocytosis. P. A. Bromberg, J. Theodore, E. D. Robin * AND W. N. Jensen,* Pittsburgh, $\mathrm{Pa}$.

Human erythrocyte $\mathrm{pH}$ was investigated by means of the indicator substance 5,5-dimethyl-2,4-oxazolidinedione (DMO), and the results compared with simultaneous measurements based on chloride distribution between cells and plasma and by direct glass-electrode $\mathrm{pH}$ measurement of packed cells lysed by a freeze-thaw procedure. In 10 simultaneous determinations by each method in six subjects without demonstrable hematologic abnormality, erythrocyte $\mathrm{pH}$ was $7.13 \pm 0.03$ (DMO), $7.15 \pm 0.05$ (electrode), and $7.25 \pm 0.03$ (chloride) at a mean plasma $\mathrm{pH}$ of $7.44 \pm 0.01$. The induction of transient moderate respiratory acidosis and alkalosis and of metabolic alkalosis by $\mathrm{NaHCO}_{3}$ infusion induced $\mathrm{pH}$ changes of similar direction and magnitude in erythrocytes and plasma. Erythrocyte $\mathrm{pH}$ was measured in five patients with active hemolytic anemias [sickle cell anemia (3), paroxysmal nocturnal hemoglobinuria (1), acquired hemolytic anemia (1)]. There were no discernible differences in $\mathrm{pH}$ between these and the control group. In contrast, three patients with hereditary spherocytosis and intact spleens had DMO-determined erythrocyte $\mathrm{pH}$ values of $7.00,6.93$, and 7.04 when plasma $\mathrm{pH}$ values were, respectively, 7.40, 7.42, and 7.41. Three additional postsplenectomy patients with hereditary spherocytosis had erythrocyte $\mathrm{pH}$ values of $7.05,7.13$, and 7.13 when plasma $\mathrm{pH}$ values were, respectively, 7.41, 7.43, and 7.37. The normal erythrocyte $\mathrm{pH}$ value of $7.13 \pm 0.03$ as determined by the DMO method implies that erythrocyte $\left[\mathrm{H}^{+}\right]$is approximately twice that of plasma. This normal $\left[\mathrm{H}^{+}\right]$gradient has been attributed to Donnan equilibrium produced by nondiffusible, negatively charged molecules within the erythrocyte. The unique finding of an increased erythrocyte-plasma $\left[\mathrm{H}^{+}\right]$gradient in four of six patients with hereditary spherocytosis may, therefore, be related to an excessive intracellular concentration of nondiffusible negatively charged particles. The possible relationship of the erythrocyte acidosis to genetically determined metabolic abnormalities remains to be investigated.

Measurements of $K^{+}$Exchange in Separated Renal Tubules. Maurice Burg, Mones Berman and Jack OrLoff,* Bethesda, Md.

A method has been developed for the separation and study of viable segments of renal tubules from rabbit renal cortex. Separation is achieved by treatment of the excised kidney with the proteolytic enzyme, col- lagenase. The resultant suspension consists principally of short lengths of proximal tubules. Preservation of respiration and active transport in the intact cells is indicated by: oxygen consumption $8.0 \pm 0.6 \mu \mathrm{L} \mathrm{mg}{ }^{-1}$ (dry wt) hour ${ }^{-1}, \mathrm{~K}^{+}$concentration $303 \pm 21 \mathrm{mEq} \mathrm{kg}{ }^{-1}$ (dry wt), and para-aminohippurate tissue to medium ratio $21 \pm 3$. The use of this preparation affords a means of directly measuring transcellular exchange rates. This has not been possible in the conventional, relatively thick, kidney-slice preparations, since penetration through the outer layers of the tissue may limit rate of exchange in the centrally located cells. In addition, use of the suspension permits better sampling and more uniform distribution of oxygen, substrates, and test substances. The steady-state transcellular $\mathrm{K}^{+}$flux rate in suspended rabbit renal tubules has been measured with $\mathrm{K}^{2}$. At least two tissue $\mathrm{K}^{+}$compartments were observed under control conditions, one large and slowly exchanging, the other small and rapidly exchanging. Addition of the cardiac aglycone strophanthidin $\left(3 \times 10^{-4} \mathrm{M}\right)$ resulted in a $98 \%$ reduction of the steady state $\mathrm{K}^{+}$flux in the major $\mathrm{K}^{+}$compartment. This observation is consistent with the known inhibitory effects of digitalis-like steroids upon active $\mathrm{K}^{+}$transport. In addition, the rate constant for $\mathrm{K}^{+}$efflux decreased $85 \%$, indicating either that the "passive" permeability to $\mathrm{K}^{+}$was decreased or, more likely, that there is in this tissue considerable facilitated or exchange diffusion of $\mathrm{K}^{+}$that is inhibited by the drug.

Visualization of Myocardial Infarcts In Vivo by Scanning. Edward A. Carr, Jr., Mary Ellen Patno and William H. Beierwaltes, Ann Arbor, Mich. (introduced by Fred M. Davenport).

Direct demonstration of myocardial infarcts in vivo has not previously been feasible. Nine dogs were subjected to ligation of the anterior descending branch of the left coronary artery under pentobarbital anesthesia and the incisions were closed; 1 to 21 days later each dog received $700 \mu \mathrm{c}$ of $\mathrm{Hg}^{203}$-labeled chlormerodrin (Neohydrin) intravenously. Five sham-operated dogs were treated similarly. All dogs were scanned in the supine position, while still alive, 1.5 to 24 hours after administration of the radioisotope. A photoscanner with a $3 \times 2$ inch crystal and 19-hole collimator was used. After scanning, each dog was sacrificed and the heart transfixed with needles through the anterior chest wall, which was then removed with the dog still in scanning position. The heart outline was projected onto the scan. Each heart was then excised, emptied of blood, and rescanned. The radioactivity of tissue samples was subsequently determined in a well-counter and the samples examined histologically. Areas of frank necrosis or of ischemia (i.e., severely compromised blood supply without frank necrosis) were clearly seen as "hot" areas of increased radioactivity in scans of living dogs. The results in sham-operated dogs were negative. The findings were confirmed in scans of excised hearts. In ligated dogs the mean relative $\mathrm{Hg}^{203}$ concentrations (concentration of $\mathrm{Hg}^{203}$ in tissue per concentration of 
$\mathrm{Hg}^{203}$ in blood) in posterior left ventricle (normal) and anterior left ventricle (infarct or ischemia) were 0.85 and 2.78, respectively. This difference is significant $(\mathrm{p}<0.025)$. In sham-operated dogs the mean relative $\mathrm{Hg}^{203}$ concentrations in posterior left ventricle (normal) and anterior left ventricle (normal) were 0.60 and 0.56 , respectively. This difference is not significant. Scans permit diagnosis of myocardial infarcts in vivo by localization of $\mathrm{Hg}^{203}$, given as chlormerodrin. The applicability of this technique to man is now being assessed.

Osmolality, $p H$, and Inulin Concentration in Proximal Tubular Fluid of the Dog. JAMES R. CLAPP AND John F. Watson, Bethesda, Md. (introduced by Robert W. Berliner).

The following experiments were undertaken to adapt micropuncture and microanalytical techniques to the study of renal function in dogs. Micropunctures have been limited to the proximal tubule, of which $50 \%$ is accessible for puncture. Tubular fluid samples have been obtained for osmolality, $\mathrm{pH}$, and inulin concentration; and urine flow, inulin, and para-aminohippurate $(\mathrm{PAH})$ clearances were simultaneously measured in the experimental and control kidney. Sixteen samples for osmolality collected during antidiuresis were isosmotic to plasma, with tubular fluid/plasma ( TF/P) ratios ranging from 0.96 to 1.04 , while urine/plasma $(\mathrm{U} / \mathrm{P})$ ratios ranged from 2.90 to 5.82 . Fourteen samples collected during water diuresis also were isosmotic to plasma with $\mathrm{TF} / \mathrm{P}$ ratios ranging from 0.98 to 1.03 when $\mathrm{U} / \mathrm{P}$ ratios ranged from 0.11 to 0.37 . $\mathrm{pH}$ determined by quinhydrone microelectrodes on 15 tubular fluid samples during antidiuresis ranged from isohydric to $0.37 \mathrm{pH}$ units below arterial blood, with the majority of the samples exhibiting some degree of acidification. Inulin concentrations determined on 11 samples by a microanthrone method, although revealing considerable variation among values for individual tubules, had $\mathrm{TF} / \mathrm{P}$ inulin ratios ranging from 1.5 to 5.5 , with the highest ratios located between 28 and $50 \%$ of the proximal tubule. In the experiments in which urine flow, inulin, and $\mathrm{PAH}$ clearances were simultaneously measured, mean decreases of 7,13 , and $10 \%$, respectively, occurred in the experimental as compared with the control kidney. It is apparent from these experiments that micropuncture studies can be performed without appreciably altering renal function. Under the conditions of these experiments, the osmolality, $\mathrm{pH}$, and inulin concentrations of proximal tubular fluid of the dog do not differ significantly from those found in rodents.

Isolation and Characterization of Amyloid Fibrils by Physical Techniques. Alan S. Cohen and Evan Calkins, Boston, Mass. and Buffalo, N. Y. (introduced by Chester S. Keefer).

Due to difficulties in the separation of amyloid from surrounding tissue, previously reported chemical charac- teristics of this substance have been based upon the comparison of massively involved amyloid liver to normal human liver. Recently, techniques have been devised to take advantage of the insolubility of amyloid and its relative hydrophilia in order to separate it from hepatic tissue. Amyloid-laden and normal livers obtained at autopsy have been homogenized at $0^{\circ} \mathrm{C}$, washed in saline, and spun five times in a refrigerated centrifuge at $5,000 \mathrm{rpm}$ for periods of 1 hour. In this fashion three distinct layers were obtained from the amyloidotic liver and two from the normal. After separation of all layers, aliquots of each were stained with phosphotungstic acid, shadowed, and examined in the electron microscope. The top layer of the amyloid liver, in contrast to the other two layers and those of the control, consisted almost entirely of fine fibrils com. parable with those seen in ultrathin sections of amyloid tissue. The calculated fibril diameter was found to be dependent upon the type of material examined (freshly separated, frozen, or dialyzed lyophilized material) and upon the type of preparation utilized for electron microscopy (unshadowed, chromium- or platinum-shadowed, or cleaved mica preparation). In all instances, individual fibrils were under $300 \AA$ in diameter. They varied considerably in length up to 5,000 ̊. X-ray powder diffraction pattern studies of all layers after dialysis and lyophilization confirmed the presence of a component in the top layer that was not demonstrable in the other two. Chemical analyses and enzyme digestion studies were also carried out on the concentrated amyloid fibrils. No differences were found by any technique in amyloid fibrils isolated from patients with the primary or secondary form of the disease.

Androgen Production and Biosynthesis by Adrenal Glands from Patients with Normal and Hyperadrenal Function. George L. Cohn and Patrick Mulrow, New Haven, Conn. (introduced by Seymour $R$. Lipsky).

Adrenal androgen production was studied in vitro with slices from 6 "normal," 7 hyperplastic, 3 adenomatous, 2 atrophic, and 2 carcinomatous adrenal glands removed from three patients with normal, and nine with hyperadrenal function. Selected flasks received either $10 \mathrm{U}$ ACTH per $\mathrm{g}$ tissue, progesterone- $\mathrm{H}^{3}$, dehydroepiandrosterone- $\mathrm{H}^{3}$, or equimolar amounts of $17 \alpha$-hydroxypregnenolone- $\mathrm{H}^{3}$ and progesterone-4- $\mathrm{C}^{14}$. Certain flasks without radioactive substrates received $\mathrm{C}^{14}$-labeled androstenedione and $11 \beta$-hydroxyandrostenedione and dehydroepiandrosterone- $\mathrm{H}^{3}$ at the conclusion of the incubation to correct for manipulative losses. The 17-ketosteroids were isolated by paper chromatography in three different solvent systems and quantitated by alkaline fluorescence or microspectrophotometry. Radioactivity was determined by liquid scintillation and planchet counting. The range of androgen steroid production $(\mu \mathrm{g} / \mathrm{g} / 3 \mathrm{hrs})$ in all tissues without $\mathrm{ACTH}$ was 
dehydroepiandrosterone, 0 to 14.3 ; androstenedione, 0 to 5.4; 11 $\beta$-hydroxyandrostenedione, 0 to 11.3. With $\mathrm{ACTH}$ it was 0 to $5.3,0$ to 12.6 , and 0 to 47.4 , respectively. ACTH resulted in a 0 to 34 -fold increase in $11 \beta$-hydroxyandrostenedione release, but an inconstant increase in androstenedione and dehydroepiandrosterone release. Adrenal androgen production was not significantly different between the "normal" and atrophic tissue, as compared with the pathological tissues. Precursor experiments revealed that 5 to $7 \%$ of $17 \alpha$-hydroxypregnenolone- $\mathrm{H}^{3}$ was incorporated into dehydroepiandrosterone; and 5 to $13 \%$ of $17 \alpha$-hydroxypregnenolone- $\mathrm{H}^{2}$ into androstenedione and $11 \beta$-hydroxyandrostenedione as compared with 0.2 to $0.8 \%$ of $\mathrm{C}^{14}$ - (or $\mathrm{H}^{3}$ ) -progesterone. In addition, dehydroepiandrosterone- $\mathrm{H}^{3}$ was incorporated into androstenedione and $11 \beta$-hydroxyandrostenedione. Radiochemical purity was verified by countercurrent distribution, paper and column chromatography of the free $\mathrm{C}^{19}$ steroids, and their 2,4-dinitrophenylhydrazone derivatives. The evidence suggests that the major adrenal androgen biosynthetic pathway in the human adult is $17 \alpha$-hydroxypregnenolone $\rightarrow$ dehydroepiandrosterone $\rightarrow$ androstenedione $\rightarrow 11 \beta$-hydroxyandrostenedione.

$A$ New Coagulation Factor Vulnerable to Thorium and Related Elements. Robert W. Colman and Benjamin Alexander,* Boston, Mass.

The unique anticoagulant action of thorium and related rare earth elements was investigated in vivo and in vitro. Colloidal thorium dioxide infused into rabbits prolonged venous blood clotting, destroyed Factor $\mathrm{V}$ in plasma, and reversibly inactivated a hitherto undescribed factor in serum. The same abnormality was rapidly and progressively produced by adding $\mathrm{ThCl}_{4}$ to serum in vitro (final concentration, $3.0 \times 10^{-4} \mathrm{M}$ ). This factor, essential for thromboplastin generation, was not Factor IX, X, XI, or XII. Destroyed also by uranium, neodymium, and lanthanum, this factor is adsorbed by $\mathrm{BaSO}_{4}$, destroyed at $50^{\circ}$ in 20 minutes, $37^{\circ}$ in 2 hours, and $4^{\circ} \mathrm{C}$ in 2 weeks. The thorium-induced defect in serum can be reversed by oxalate or citrate, both of which bind thorium. The entity has been separated from other coagulation factors by $\mathrm{BaSO}_{4}$ adsorption, citrate elution, ammonium sulfate fractionation, and starch gel electrophoresis. An assay procedure has been developed, and the factor was found to be profoundly depressed in patients treated with coumarin derivatives, in some patients with liver disease, and in newborn infants. Unlike Factors VII and X, it is not elevated in pregnancy. Its level under physiological and pathological circumstances does not parallel the concentration of other serum clotting components. The data indicate the existence of a new factor essential for thromboplastin generation, and suggest an important relationship between the atomic structure common to these elements and a structural site crucial to the biological activity of this factor.
The Importance of Conjugation with Glutathione on Sulfobromophthalein (BSP) Transport from Blood to Bile. Burton Combes, Dallas, Tex. (introduced by Gladys Fashena).

An examination of the effect of glutathione on BSP movement from blood to bile was undertaken to determine whether conjugation of glutathione with BSP is important for, or merely incidental to, the hepatic uptake and biliary excretion of BSP. Advantage was taken of the finding that hepatic glutathione content in the rat decreased $81 \%$ (from 8.3 to $1.6 \mathrm{mg} / 100 \mathrm{~g}$ body weight) after 2 days on a protein-free diet. Control rats excreted more BSP into bile than did glutathionedeficient rats with intravenous doses of BSP (5 to $10 \mathrm{mg} / 100 \mathrm{~g}$ ). Maximal BSP excretion in bile collected for 45 minutes of 4.25 per $100 \mathrm{~g}(3.56 \mathrm{mg}$ conjugated, $0.69 \mathrm{mg}$ free $\mathrm{BSP}$ ) was reached in control animals when $10 \mathrm{mg}$ per $100 \mathrm{~g}$ was injected. Only $2.94 \mathrm{mg}$ BSP per $100 \mathrm{~g}$ was excreted in the glutathionedeficient rats at the dose of $10 \mathrm{mg}$ per $100 \mathrm{~g}$. The decrease in total BSP output was accounted for by a fall in excretion of conjugated BSP, $1.99 \mathrm{mg}$ conjugated, $0.95 \mathrm{mg}$ free. Conjugation was not necessary for hepatic uptake of BSP. The amount of BSP taken up by the liver of rats with ligated common bile ducts was the same in control and in glutathione-deficient animals. Furthermore, the extent of BSP uptake in these livers was not affected, although the proportion of conjugated BSP contained in the liver increased over a 45-minute interval. In other studies, analysis of the distribution of BSP compounds contained in liver with those just excreted into bile revealed that conjugated BSP was preferentially excreted. Glutathione-deficient rats contained less conjugated BSP in liver and excreted less conjugated BSP into bile than did control animals. These observations indicate that conjugation of BSP with glutathione is an important determinant of the maximal rate at which BSP is transferred from blood to bile. Transport from the liver cell into bile appears to be the conjugation-dependent step, conjugated BSP being transported more rapidly than is free BSP.

Anatomic Structure of Lymphatic Tissues and Immune Function. Charles C. Congdon,* Oak Ridge, Tenn.

For many years attempts have been made to relate histologic changes in lymphatic tissues to function of the immune mechanism. The task of demonstrating that immune function resides in lymphatic tissues was finished earlier and has been repeatedly confirmed. Immune function outide the lymphatic tissues we can attribute to disbursement of mobile cells from these tissues. Within the lymphatic tissues new histologic studies seem to indicate clearly that immune function begins in the cortex of lymph nodes and the white pulp of spleen. Changes in the medulla of lymph nodes and red pulp of spleen depend on the use of certain kinds of antigens and follow the primary changes in cortical and white pulp areas of the lymphatic tissues. The primary 
changes are qualitatively the same after all kinds of antigenic stimulation. For this reason the primary histologic changes constitute a potential method for diagnosing the presence of immunologic function. Within the cortex of lymph nodes and white pulp of spleen it is necessary to account for changes after antigenic stimulation in marginal zone cells, lymphocytes, germinal centers, tingible bodies, and stromal elements. The present work indicates that the germinal center is the major histologic structure associated with immune function. The tingible body is also a key anatomic structure whose function we do not understand, but perhaps is concerned with the recognition of the antigenic material through phagocytosis of lymphocytes and granulocytes. The present work also requires a precursor cell for the germinal center. The two possibilities seem to be a fixed reticulum cell or the lymphocyte. The ultimate precursor cell may be the thymus. All questions of immune function and disease will need eventually to deal with these several anatomic structures.

Vitamin- $B_{12}$ Binding by Normal Human Serum. BerNARD A. COOPER AND Louis Lowenstein, Montreal, Canada (introduced by David V. Bates).

Since the demonstration that vitamin $B_{12}$ in human serum is bound to protein, numerous studies have attempted to characterize the protein and measure the binding capacity. Diverse techniques have been used to study this phenomenon, and the values obtained have varied with the techniques used. Consequently, widely divergent values for cyanocobalamin-binding capacity of human serum have been reported. The physiological importance of this binding is unknown, although we have reported recently that much larger quantities of cyanocobalamin bound to human serum protein than of free cyanocobalamin are taken up by certain tumor cells incubated in vitro. The rate of clearance from the plasma in vivo of injected cyanacobalamin is altered by binding to the binding protein of serum. Using several techniques simultaneously, we have studied cyanocobalamin binding by normal serum, to evaluate techniques and to reconcile the differing results reported previously. The values obtained for cyanocobalamin-binding capacity of normal serum, measured by a modified charcoaladsorption technique, paper electrophoresis, ultrafiltration, and the stimulation of uptake of cyanocobalamin by Ehrlich ascites cells in vitro, are approximately equal. The much lower binding capacity determined with Euglena gracilis seems to be caused by the reduced affinity of the binding protein for cyanocobalamin at $\mathrm{pH} 3$ to 4 . The cyanocobalamin-binding capacity at pH 3 to 4, measured by the modified charcoal-adsorption technique, is changed to a value comparable to that obtained with E. gracilis. Physiochemical studies indicate that, at physiological $\mathrm{pH}$ and temperature, the binding protein binds cyanocobalamin tenaciously, and preferentially to pseudovitamin $B_{12}$. Under carefully controlled conditions, normal human serum has a finite binding capacity for vitamin $B_{12}$ and specific physical, chemical, and biological properties.

Familial Pyridoxine-responsive Anemia. H. Brooks Cotton, Fairfield, Ala. AND JoHN W. Harris,* Cleveland, Ohio.

Severe pyridoxine-responsive anemia has been demonstrated in a 44 year old white female; moderately severe, pyridoxine-responsive anemia in her 43 year old brother; and hyperferremia in three (one female) of five children ( 6 to 18 years). The patients' mother, sister, six paternal, and two maternal cousins showed no hematologic abnormalities and serum iron values were normal; their father died of "cirrhosis" (no records available). Anemia of 32 years is documented for the female with exacerbations during pregnancies requiring nine transfusions; the erythrocytes have consistently been described as hypochromic and microcytic despite over $2 \mathrm{~g}$ of parenteral iron. Pyridoxine therapy resulted on three occasions in subjective improvement, reticulocytosis, and hemoglobin increases $(7 \mathrm{~g} / 100 \mathrm{ml}$ before, $13 \mathrm{~g} / 100 \mathrm{ml}$ after). The serum iron was consistently elevated to the 200 to $300 \mu \mathrm{g} / 100 \mathrm{ml}$ range during relapse, and the erythrocyte $\mathrm{Cr}^{61}$ half-life was 24 days with no sequestration by the moderately enlarged spleen. Anemia of varying severity ( 8 to $11 \mathrm{~g} \mathrm{Hb} / 100 \mathrm{ml}$ ) for 15 years is documented for the brother. The erythrocytes have appeared slightly hypochromic and microcytic, the serum iron elevated to $200 \mu \mathrm{g}$ per $100 \mathrm{ml}$. Pyridoxine therapy was followed by mild reticulocytosis, hemoglobin increase $(10 \mathrm{~g} / 100 \mathrm{ml}$ to $14 \mathrm{~g} / 100 \mathrm{ml})$, and fall to normal range in the serum iron; the spleen became no longer palpable (formerly 3 finger-breadths enlarged). Both patients had hematologic responses to oral or parenteral administration of various forms of pyridoxine but not to the usual hematopoietic agents. The erythrocyte abnormalities persist despite achievement of normal hemoglobin levels but no abnormalities in tryptophan metabolism have been found upon repeated loading tests. The hemoglobin showed normal electrophoretic migration and no elevation of $A_{2}$ or $F$ hemoglobin. No anemia or erythrocyte abnormalities were found in the children. In the one child tested by pyridoxine administration the serum iron fell from 254 to $139 \mu \mathrm{g}$ per $100 \mathrm{ml}$.

Fatty Acid Composition of Erythrocyte Phospholipids in Anemic Patients. W. T. Dabney, III and C. W. Chamberlain, Richmond, Va. (introduced by G. Watson James, III).

The fatty acid composition of the phospholipid fraction of the erythrocytes from 40 individuals has been investigated. Included are 10 normal males, 9 normal females, and 21 patients with a hemoglobin less than $10 \mathrm{~g}$ per $100 \mathrm{ml}$. The total lipid of saline-washed erythrocytes from heparinized venous blood was extracted and the phospholipid fraction separated on a silicic acid column. An analysis of the methyl esters of the fatty acids was performed by gas-liquid phase chromatography with ethylene glycol succinate polyester as the stationary 
phase. At least 17 fatty acids are associated with the erythrocyte phospholipids. Palmitic, stearic, oleic, linoleic, and arachidonic acid are the major fatty acids present. No qualitative difference was observed in the fatty acid composition of the normal or anemic group. A quantitative comparison of the per cent composition of the major fatty acids revealed a significant difference. Analysis of the data revealed a significant decrease $(p<0.001)$ in the percentage of linoleic acid in erythrocytes from anemic subjects when compared with normal individuals. No significant difference was found in the other fatty acids with the exception of a slight increase in oleic $(p, 0.01)$. There is a significant positive correlation $(r=0.715, p<0.001)$ between the hemoglobin concentration and percentage of linoleic acid if the two study groups are combined, but no significant correlation was found within the anemic group or normal group when analyzed separately. Current studies indicate a similar quantitative change in the fatty acid composition of the plasma phospholipids and neutral lipids in severe chronic anemia. The decrease in linoleic acid does not appear to be related to the etiology or morphologic characteristics of the anemia.

Hemodynamic Consequences of Oxygen Breathing in Left Ventricular Failure. WALTER J. DALY AND RoY H. BEHNKE, Indianapolis, Ind. (introduced by John Hickam).

Breathing oxygen decreases the heart rate and cardiac index of resting normal man. Using standard techniques of cardiac catheterization and an indocyanine green dilution method, we studied the hemodynamic effects of hyperoxia in 15 nonhypoxemic patients with left ventricular failure (mean arterial oxygen saturation, $94.6 \pm$ $2.3 \%$ ). Air or oxygen was breathed in random sequence. Oxygen breathing was associated with a decrease in cardiac index from $2.36 \pm 0.71$ to $2.07 \pm 0.45$ $\mathrm{L}$ per minute per $\mathrm{m}^{2}(\mathrm{p}<0.001)$; heart rate, $84 \pm 25$ to $81 \pm 25$ beats per minute $(p<0.001)$; stroke index, $33 \pm 19$ to $30 \pm 18 \mathrm{ml}$ per $\mathrm{m}^{2}(\mathrm{p}, 0.005)$. The effects on $\mathrm{A}-\mathrm{V} \quad \mathrm{O}_{2}$ difference $(6.87 \mathrm{vol} \%), \mathrm{O}_{2}$ delivery index (arterial $\mathrm{O}_{2}$ content $\times$ cardiac index $=438 \pm 163 \mathrm{ml} \mathrm{O}_{2} /$ $\left.\min / \mathrm{m}^{2}\right)$, systemic mean arterial pressure $(106 \pm 13 \mathrm{~mm}$ $\mathrm{Hg})$, pulmonary artery mean pressure $(33 \pm 11 \mathrm{~mm} \mathrm{Hg})$, wedge pressure $(20 \pm 6 \mathrm{~mm} \mathrm{Hg})$ were not statistically significant. Contrary to observations made in other situations of pulmonary hypertension, oxygen breathing did not alter pulmonary vascular resistance $(296 \pm 176$ dynes-sec- $\mathrm{cm}^{-6}$ ). In normal man the decrease in cardiac index during oxygen breathing occurs without a change in stroke index. In heart failure, the chronotropic effect of hyperoxic breathing remains effective; furthermore, a small but consistent decrease in stroke index is observed. These data suggest that oxygen breathing further decreases the already reduced cardiac output of left ventricular failure without impairing the delivery of oxygen to the tissues. Such changes would be expected to reduce the work of the failing heart without further impairing its ability to meet the demands placed upon it.
The Formation of Free Hydroxyproline by Cartilage. William H. Daughaday* and Ida Kozak Mariz, St. Louis, Mo.

Formation of free hydroxyproline by cartilage has been examined because of its relevance to the important reaction involved in collagen synthesis by which proline is hydroxylated. Segments of costal cartilage from rapidly growing rats were incubated in an enriched medium containing L-proline-U-C $\mathrm{C}^{\mathbf{1 4}}$. After incubation for 24 hours, free hydroxyproline in the medium and bound hydroxyproline in whole cartilage or cartilage fractions has been isolated by Dowex- 50 chromatography and its specific activity determined by the method of Prockop, Udenfriend and Lindstaedt. Between 8 and $10 \mu \mathrm{g}$ of free hydroxyproline per $100 \mathrm{mg}$ of wet cartilage was released into the medium. This hydroxyproline had a specific activity 20 times that of hydroxyproline of collagen soluble in $0.45 \mathrm{M} \mathrm{NaCl}$ and 65 times that of hydroxyproline from collagen insoluble in $0.45 \mathrm{M} \mathrm{NaCl}$. Cortisol (2 to $10 \mu \mathrm{g}$ / $\mathrm{ml}$ inhibited incorporation of $\mathrm{C}^{\mathbf{1 4}}$ into cartilage-bound hydroxyproline by about $30 \%$, but caused a small (12\%) stimulation of labeling of medium free hydroxyproline. Conclusion: Free hydroxyproline formed by cartilage in the system is not derived from soluble collagen but probably arises from an activated hydroxyproline intermediate that exists prior to the stage of peptide synthesis. Formation of this intermediate apparently is not inhibited by cortisol, although cortisol appears to inhibit collagen synthesis.

The Effect of Adenosine Derivatives on Ion Transport and Energy Metabolism in the Toad Bladder. RoBerT P. Davis, Mitzy Canessa-Fischer and Chester M. Edelmann, JR., New York, N. Y. (introduced by I. Herbert Scheinberg).

The energy for active transport of sodium is derived from high-energy phosphate bonds and at least in part from cellular respiration. Since the major regulator of cellular respiration is adenosine diphosphate, we hoped to clarify the linkage between transport of sodium and cellular respiration by studying the effects of this nucleotide on these processes in the urinary bladder of the toad. Transport of sodium was measured by shortcircuit current and respiration polarographically by the vibrating platinum electrode. Reduced pyridine nucleotide, through which high-energy phosphate bond formation is coupled to the reactions of cellular respiration, was identified in the intact functoning bladder by its fluorescence. Three phenomena were observed when adenospine diphosphate was applied to the toad bladder: 1) respiration was acclerated, 2) the concentration of reduced pyridine nucleotide was decreased, and 3 ) there was a pronounced decrease in the transport of sodium. In contrast, adenosine, adenosine 5'-monophosphate (AMP) and cyclic 3',5'-AMP produced a significant initial increase in transport of sodium, presumably during initial phosphorylation reactions. There then followed a decrease in the transport of sodium comparable with 
that produced by adenosine diphosphate. Since adenosine diphosphate leads to opposite changes in respiration and transport of sodium, a direct linkage through a redox pump between the electron transport aspect of respiration and transport of sodium appears unlikely. In contrast, the changes in transport of sodium produced by adenosine derivatives parallel closely the changes in the concentration of reduced pyridine nucleotide. From these results, we infer that an intermediate in the reaction sequence between reduced pyridine nucleotide and the phosphorylation of adenosine diphosphate is the point of coupling between energy metabolism and ion transport.

Evidence for a Second Iron-binding System in Plasma. Raymond J. Dern, Alberto Monti and Michael F. Glynn, Chicago, Ill. (introduced by Richard L. Landau).

Plasma iron was found to be cleared at two different rates that are apparently unrelated to feedback kinetics: a rapidly cleared iron fraction accepted as transferrinbound, and a slowly cleared iron fraction. Radioironlabeled plasma was obtained from donors 2 days after injection of $\mathrm{Fe}^{59}$. This was incubated with $\mathrm{Fe}^{55}$ in the accepted method of labeling transferrin and then injecting the doubly-labeled plasma into recipients. The disappearance rate of $\mathrm{Fe}^{50}$ in the recipient was one-half to one-third of that observed for $\mathrm{Fe}^{\mathrm{s5}}$. Since the recipient had no tracer in the labile erythropoietic iron pool, the slow rate was not related to feedback kinetics. Recipients were selected to provide a spectrum of serum iron levels and storage iron. In three donor-recipient studies, the clearance rate of the second binding component was not related to the iron status of the recipients but appeared to be somewhat faster with plasma from an iron-poor donor. When plasma was obtained from donors within 2 hours after oral or intravenous administration of $\mathrm{Fe}^{58}$ and incubated with $\mathrm{Fe}^{\mathrm{ss}}$ as before, the disappearance rates in the recipients for both isotopes were the same. It is suggested that plasma iron is bound in two ways: the transferrin(s) system for injected and absorbed iron; and a second iron-binding system representing, in part, recirculating iron. The results of current studies of erythrocyte iron utilization and differential behavior of the two forms of iron transport will be discussed.

Abnormal Neural Regulation of Vasopressin and Aldosterone Secretion in a Patient with Cerebral Hyponatremia. Joseph F. Dingman, Akira Arimura, Ralph E. Peterson* and Robert G. Heath. Boston, Mass., New York, N. Y. and New Orleans, La.

A male patient with cerebral metastases from bronchogenic carcinoma developed symptomatic hyponatremia which was partially corrected by high $\mathrm{Na}$ and ad lib fluid intake, and completely reversed by high $\mathrm{Na}$ and low fluid regimen. Low $\mathrm{Na}$ intake resulted in continued renal $\mathrm{Na}$ loss and return of hyponatremia which could not be prevented by fluid restriction. Ad lib hydration accelerated the onset of hyponatremia with $\mathrm{Na}$ restriction and resulted in higher $\mathrm{Na}$ excretion than during dehydration. Urinary aldosterone was depressed during high $\mathrm{Na}$ intake but failed to increase normally on low $\mathrm{Na}$ diet. The adrenals failed to respond to methopyrapone. $\mathrm{ACTH}$ administered intravenously during low $\mathrm{Na}$ regimen produced a normal steroidal response and a marked rise in aldosterone to levels observed in Na-depleted normal subjects. These findings suggest a derangement in the physiological regulation of aldosterone secretion related to a disturbance in the neural control of ACTH secretion. The typical abnormal response to water loading was apparent even when serum $\mathrm{Na}$ and body hydration were within normal range. Vasopressin was extracted from acidified plasma, purified on IRC-50 columns and silicic acid-impregnated glass paper chromatograms, and specific vasopressin zones were bioassayed in the alcohol-anesthetized hydrated rat. Plasma vasopressin was five times normal on ad lib hydration, failed to fall with water loading, and was much in excess of the normal response after intravenous nicotine. Curiously, the patient maintained normal serum $\mathrm{Na}$ on ad lib $\mathrm{Na}$ and water intake in the last few weeks of life. Postmortem examination revealed bilateral destruction of the septal region and subtotal destruction of the adrenals, but the hypothalamic-pituitary region was unaffected. These findings suggest that the hyponatremia resulted from involvement of subcortical centers regulating secretion of hypothalamic, pituitary, and adrenal hormones which control the plasma concentration and the renal excretion of $\mathrm{Na}$ and water.

Demonstration of an Exchange between Free and Intrinsic Factor-bound Vitamin $B_{12}$. RoBerT M. DoNaldson, JR. Jay H. Katz and Josè̀h D. DiMase, Boston, Mass. (introduced by M. B. Strauss).

The source of "intrinsic factor" (IF) used in these studies was neutralized human or rat gastric juice, which was shown to promote the absorption of vitamin $\mathrm{B}_{12}$ in patients with pernicious anemia or in the gastrectomized rat, respectively. Gastric juice, the binding capacity of which had been saturated with $\mathrm{Co}^{80}$-labeled vitamin $\mathrm{B}_{12}$, was mixed with free $\mathrm{Co}^{57}$-labeled vitamin $B_{12}$. When the IF-bound $B_{12}$ was subsequently separated from free $B_{12}$, a significant quantity of the initially free $\mathrm{Co}^{57} \mathrm{~B}_{12}$ was now bound, while an equal amount of the originally bound $\mathrm{Co}^{60} \mathrm{~B}_{12}$ had become free. Exchange between the two isotopes was demonstrated whether bound and free vitamin $B_{12}$ were separated by dialysis, by starch gel electrophoresis, or by dextran gel filtration. The rate of exchange was temperaturedependent. When free and bound vitamin $B_{12}$ were mixed in equal amounts at $37^{\circ} \mathrm{C}, 40 \%$ of the theoretically possible exchange occurred within 2 hours. Neither the binding capacity of the gastric juice nor the electrophoretic mobility of the bound radioactivity was altered after exchange had occurred. Schilling tests were performed in pernicious anemia patients with mixtures of equal amounts of free $\mathrm{Co}^{80} \mathrm{~B}_{12}$ and IF-bound 
$\mathrm{Co}^{87} \mathrm{~B}_{12}$. When these mixtures were incubated at $37^{\circ} \mathrm{C}$ before administration, the urinary excretion of the initially free $\mathrm{Co}^{80} \mathrm{~B}_{12}$ was regularly increased, while excretion of the originally bound $\mathrm{Co}^{57} \mathrm{~B}_{12}$ was correspondingly decreased. Previous in vivo and in vitro isotope studies concerned with vitamin $\mathrm{B}_{12}$ absorption and IF function have assumed that the specific activity of IFbound vitamin $B_{12}$ is constant. The occurrence of exchange between free and IF-bound vitamin $B_{12}$ may complicate the quantitative interpretation of such studies.

Control of Cardiac Output in Anemia. Martin Duke and Walter H. Abelmann,* Boston, Mass.

Fifteen anemic patients, 31 to 86 years old, were studied supine, before and after treatment with 5.9 and $10.9 \mathrm{~g}$ hemoglobin per $100 \mathrm{ml}$, respectively. Measurements (Evans blue dye) of cardiac output and total blood volume (TBV) were made, injecting through a polyethylene catheter into axillary or caval vein and withdrawing via brachial artery. After treatment, there were significant changes in the mean differences $(p<$ 0.01 ) of the following: cardiac index (CI) decreased from 4.73 to $3.44 \mathrm{~L}$ per minute per $\mathrm{m}^{2}$, heart rate (HR) decreased from 82.8 to 69.5 beats per minute, stroke index (SI) fell from 57.6 to $49.5 \mathrm{ml}$ per beat per $\mathrm{m}^{2}$, total systemic resistance (TSR) rose from 1,017 to 1,526 dynes-sec- $\mathrm{cm}^{-5}$, and TBV (13 cases) rose from 4.55 to $5.20 \mathrm{~L}$. Central blood volume (CBV) remained unchanged, 1.69 to $1.62 \mathrm{~L}$. Accordingly, CBV/TBV fell from 0.378 to $0.316(p<0.01)$. Four anemic patients were studied in supine and sitting positions. Uniformly, decreases occurred in CI from 4.45 to $3.41 \mathrm{~L}$ per minute per $\mathrm{m}^{2}$, SI from 52.9 to $37.9 \mathrm{ml}$ per beat per $\mathrm{m}^{2}$, TBV from 3.35 to $3.12 \mathrm{~L}, \mathrm{CBV}$ from 1.21 to $0.93 \mathrm{~L}$, and $\mathrm{CBV} / \mathrm{TBV}$ from 0.360 to 0.299 , HR rising in three cases. In eight normal subjects and six anemic patients, methoxamine was administered intravenously ( 0.3 to $0.6 \mathrm{mg} / \mathrm{min}$, total 8.5 to $20.7 \mathrm{mg}$ ). In normals, CI remained unchanged, $\mathrm{HR}$ fell, $\mathrm{SI}$ rose from 59.6 to $76.0 \mathrm{ml}$ per beat per $\mathrm{m}^{2}, \mathrm{CBV}$ rose $11 \%$ from 1.83 to $2.03 \mathrm{~L}$, and TSR increased from 1,046 to 1,286 dynes$\mathrm{sec}-\mathrm{cm}^{-8}$. In anemia, CI fell from 5.73 to $4.62 \mathrm{~L}$ per minute per $\mathrm{m}^{2}$, HR fell, SI rose from 66.6 to 74.9 $\mathrm{ml}$ per beat per $\mathrm{m}^{2}, \mathrm{CBV}$ was unchanged, and TSR rose from 801 to 1,046 dynes-sec- $\mathrm{cm}^{-5}$. The circulation in anemia is characterized by tachycardia, increased stroke volume, decreased TSR, hypovolemia, and shift of blood centrally. This response appears obligatory but is partially reversible by changes of position or by the vasopressor methoxamine.

Urinary Total Hydroxyproline as an Index of Connective Tissue Turnover in Bone. TheODORE A. Dull, Lynn Causing and Philip H. Henneman,* Jersey City, N. J.

Hydroxyproline occurs in the body only in collagen and connective tissue; on a meat- and gelatin-free diet urinary total hydroxyproline may reflect connective tissue turnover. Using the method of Prockop and Udenfriend, we found that the daily urinary total hydroxyproline was 27 to $96 \mathrm{mg}$ in five prepubertal children and 8 to $31 \mathrm{mg}$ in five normal adults on a meat- and gelatinfree diet. Seven adults with diseases not known to involve connective tissue also excreted 9 to $27 \mathrm{mg}$ hydroxyproline daily. In three hypopituitary patients, aged 15,21 , and 22 years, urinary hydroxyproline was 34,45 , and $42 \mathrm{mg}$, respectively. Eight days' treatment with human growth hormone in the 22 year old hypopituitary patient increased urinary hydroxyproline from 42 to $72 \mathrm{mg}$. A patient in the fifth month of pregnancy excreted $45 \mathrm{mg}$ daily. A patient with severe hyperthyroidism excreted $91 \mathrm{mg}$ daily. In a patient with severe osteitis urinary hydroxyproline decreased from 100 to $61 \mathrm{mg}$ in the 5 months following cure of hyperparathyroidism. Highest values (116 mg daily) were noted in a patient with extensive, active Paget's disease of bone. These data suggest that 24-hour urinary total hydroxyproline excretion during a meat- and gelatin-free diet may provide as sensitive an index of bone collagen turnover as does calcium excretion of bone mineral metabolism.

Osmotic Water Transfer Across a Vasopressin-sensitive and an Artificial Membrane. LAURENCE E. EARLEY, Boston, Mass. (introduced by Laurence B. Ellis).

It has been suggested that net water transfer along osmotic gradients, exceeding predicted differences in diffusion across semipermeable membranes, results from hydraulic (bulk) flow through porous openings in the barrier. Vasopressin is thought to increase hydraulic flow along osmotic gradients by increasing pore size in sensitive membranes. If so, such hydraulic flow should produce some increase in water movement in the direction of large net transfers. Total water $-\mathrm{H}^{2}$ movement from mucosa to serosa was measured, and total movement from serosa to mucosa calculated, from the isotopic measurement and simultaneous net transfer (weight change) across toad bladder pouches in vitro. With isotonic media, movement in each direction averaged $459 \mu \mathrm{l}$ per $\mathrm{cm}^{2}$ per hour and net transfer was near zero. Osmolality of the mucosal or serosal medium, or both, was altered to produce gradients of $0,+165$, or $-165 \mathrm{mOsm}$ per $\mathrm{kg}$, and each result was compared to its own control (isotonic media). Net water transfers did not correlate with increased water movement in the direction of the predicted hydraulic flow. An inverse relationship existed between osmolality and water movement from the altered medium. This relationship accounted for net transfer, while water movement from the medium (of constant osmolality) changed little. A similar relationship accounted for net water transfer along osmotic gradients (sucrose) across cellulose dialysis tubing. Without gradients, solute suppressed water $-\mathrm{H}^{3}$ movement in an order inversely related to permeation of the solute species (sucrose $>$ glucose $>$ urea); 2 molal sucrose depressed the movement of 
water- $\mathrm{H}^{3}$ and urea- $\mathrm{C}^{14} 75$ and $70 \%$, respectively. These data do not support the concept of hydraulic flow through pores and suggest that large net transfers of water and unequal fluxes of permeant solute across such membranes may result from asymmetric diffusional permeability, produced by unequal concentrations of impermeant solute on the two sides of the membrane.

A Kinetic Analysis of the Antidiuretic Action of Vasopressin. IsIDore S. Edelman* and Martin J. Petersen, San Francisco, Calif.

The discovery of the similarity in the effect of vasopressin on the skin and urinary bladder of amphibia and the mammalian kidney made it possible to define the kinetics of vasopressin action in an isolated system, uncomplicated by other physiological events. A simple chamber was designed for measuring microflows of water across a sheet of cells continuously and accurately, enabling measurement of the magnitude and the timecourse of the antidiuretic response of isolated toad bladders to vasopressin. At fixed osmotic gradients of $200 \mathrm{mOsm}$ per $\mathrm{kg} \mathrm{H}_{2} \mathrm{O}$, the minimal concentration of vasopressin (Pitressin) necessary to produce an increase in the rate of water flow across the toad bladder was 0.5 to $1 \mathrm{mU}$ per $\mathrm{ml}$. Dose-response curves revealed typical saturation kinetics, the maximal response being attained at concentrations $>10 \mathrm{mU}$ per $\mathrm{ml}$. A simple hypothesis served to describe these results. The assumptions were that: 1) the rate of flow at a fixed osmotic gradient was linearly proportional to the number of vasopressin molecules bound per $\mathrm{cm}^{2}$ of cell surface, and 2) the binding of vasopressin to the cell membrane was a one-step reversible reaction. Based on these assumptions, it was predicted and shown in 12 experiments that the action of vasopressin is characterized by a linear dependence of the reciprocal of the rate of osmotic flow on the reciprocal of the vasopressin concentration in the extracellular medium. The coefficients in this equation provide independent measures of empirical vasopressin-membrane binding constants and of the membrane binding capacity for vasopressin. In addition, these studies confirmed that the action of vasopressin is reversible and that exposure to high concentrations of vasopressin induces resistance to the antidiuretic action of the hormone. The kinetic analysis provides a method for evaluating the mechanism of action of vasopressin inhibitors and of intermediates in antidiuretic pathways and a quantitative bioassay of antidiuretic activity.

Selective Inhibition of Enterovirus Nucleic Acid Synthesis by 2-( $\alpha$-Hydroxybenzyl)-Benzimidazole (HBB). Hans J. Eggers and Igor Tamm,* New York, N. Y.

HBB is one of the few synthetic chemical compounds that are capable of inhibiting virus multiplication at nontoxic concentrations. Most human enteroviruses are susceptible to inhibition by HBB, but members of other groups of viruses are insusceptible. An investigation of the precise mechanism of action of $\mathrm{HBB}$ was undertaken, since it appeared that HBB might assist in identifying a particular step in the reproduction of enteroviruses and provide an approach to the study of the chemical basis of virus specificity. Evidence has been obtained that HBB has no effect on the early phases of enterovirus-host cell interaction (i.e., adsorption and penetration). However, HBB does interfere with one of the later processes-synthesis of virus precursor materials or assembly of virus particles-as shown by the fact that the compound inhibited virus multiplication even when added during the exponential growth of the virus. A detailed study of the effects of HBB on the synthesis of infective viral RNA has been carried out with three viruses, ECHO 12, Coxsackie A9, and Coxsackie B4. HBB was used at a concentration of $0.2 \mathrm{mM}$, which is sufficient to stop virus production. The compound prevented the synthesis of infective viral RNA, but had no effect on host cell RNA synthesis. It should be emphasized that $\mathrm{HBB}$ had no direct irreversible inactivating effect on the infectivity of viral RNA, nor did it interfere with its uptake by cells. In HBB-treated cells, in which replication of infective viral RNA was inhibited, the synthesis of complement-fixing antigen was also inhibited. The results suggest that $\mathrm{HBB}$ inhibits enterovirus multiplication by inhibiting the synthesis of viral RNA. However, the possibility has not been excluded that HBB may also have other effects on the viral reproductive process.

Quantitative Differences in Osteoporosis-producing Action of Corticoids. Eugene EIsenberg AND GIlbert S. Gordan,* San Francisco, Calif.

The adverse results (so-called "side effects") of corticoid therapy for various disease are, in fact, due to expected physiologic actions of these compounds in the doses required. One of these effects is increased breakdown of bone which results in osteoporosis and fracture. Previous chemical modifications of steroid structure have failed to eliminate this undesirable property, although another unwanted action, sodium retention, has been quantitatively reduced (prednisone), or even reversed (triamcinolone). Measurement of skeletal dynamics with stable strontium as a tracer showed that the osteoporosis of Cushing's disease or of corticoid treatment is characterized in its earliest phase by increased urinary loss of the bone-seeking tracer without decrease in bone deposition rate. After prolonged treatment or late in the disease, the rapidly miscible volume of tracer distribution shrinks and the bone deposition rate falls. Thus, the corticoids are primarily "catabolic" and secondarily "antianabolic." Administration of large doses of corticoids (cortisol $120 \mathrm{mg} /$ day; prednisone 20 to $30 \mathrm{mg} /$ day; triamcinolone 12 to $20 \mathrm{mg} /$ day; or dexamethasone $3 \mathrm{mg} / \mathrm{day}$, each administered to 6 or more normal subjects) increased urinary excretion of the tracer. This effect was significantly reduced by 
halving the dose of dexamethasone. A difluorinated corticoid, 6- $\alpha$-fluorotriamcinolone, with approximately twice the anti-inflammatory activity of triamcinolone or prednisone, was similarly studied. In a dose of 12 mg per day this compound did not increase the urinary excretion rate to the extent found with large doses of the other corticoids but only to the lesser degree obtained with the small dose of dexamethasone. These findings signify that the difluorinated steroid is less likely to induce osteoporosis in usual maintenance doses than are the other anti-inflammatory steroids.

Postural Changes in Plasma Volume in Hypoalbuminemic Subjects. Seymour EISEnberg, Dallas, Tex. (introduced by Ben Friedman).

Transcapillary fluid movement is governed largely by the net pressure generated by opposing forces of hydrostatic and plasma oncotic pressure. This study was undertaken to investigate postural alterations in plasma volume (PV) under circumstances of a distorted Starling equilibrium; i.e., in hypoalbuminemic individuals. PV was calculated from the $\mathrm{Cr}^{51}$ red cell mass and the hematocrit. Cirrhotic and nephrotic subjects were studied during recumbency and after quiet standing. By comparing data obtained from venous sampling sites located at and below the atrial level and from arterial samples evidence was obtained, indicating that dependency of the venous sampling site leads to local transudation and, hence, to spurious changes in PV. In 19 normal subjects, standing resulted in a $279 \mathrm{ml}(11 \%)$ decline in PV (arm dependent), $216 \mathrm{ml}$ or $8 \%$ (vein at atrial level), and $183 \mathrm{ml}$ or $7 \%$ (arterial). In 23 hypoalbuminemic patients assumption of the erect posture resulted in a $621 \mathrm{ml}$ or $18 \%$ decrease in PV (arm dependent), $523 \mathrm{ml}$ or $16 \%$ (arm at atrial level), and $606 \mathrm{ml}$ or $16 \%$ (arterial) ( $p<0.001)$. Serum protein concentration consistently rose, indicating fluid loss rather than distributional shifts. The usual decline in PV associated with standing is exaggerated in hypoalbuminemic subjects. Losses of this magnitude undoubtedly affect cardiac output and arterial blood volume. The arm position data suggest that hematocrit variations occur throughout the body in response to hydrostatic changes; attention to position of the sampling site is essential to avoid errors induced by local fluid transudation.

\section{Erythropoietin and Testosterone In Vitro. AlLAN J. ERsLev,* Philadelphia, Pa.}

Whole anemic serum has not been found to stimulate the uptake or utilization of isotopically-labeled building blocks in bone marrow suspensions. The recent availability of purified sheep-erythropoietin has made it possible to determine with greater specificity whether or not erythropoietin is active in vitro. Bone marrow suspensions were prepared from surgically removed human ribs or rabbit bones and aliquot samples were incubated for 21 and 45 hours at physiologic tempera- ture, $\mathrm{pH}, \mathrm{pO}_{2}$, and $\mathrm{pCO}_{\%}$. Erythropoietin was added and the cellular uptake of $\mathrm{Fe}^{50}$ was determined. It was found that erythropoietin increases the uptake of $\mathrm{Fe}^{50}$ if, 1) the cellular concentration is low; 2) the suspending fluid contains appreciable amounts of tissue culture medium. The optimal results were obtained at cellular concentrations of 2,000 cells per $\mathrm{mm}^{3}$ and a suspending medium of two-thirds Eagle's MEM and one-third normal serum. Under these conditions erythropoietin induced a definite log-dose response with 2.5 cobalt units per $\mathrm{ml}$, producing an increase of 20 to $30 \%$ after 21 hours' incubation and 40 to $100 \%$ after 45 hours' incubation. Crystalline testosterone added to the same system also increased the cellular iron uptake but only during the first 21 hours' incubation. These results suggest that testosterone may act by accelerating the maturation or division of cells present in the bone marrow suspension whereas erythropoietin has a delayed effect, possibly through the creation of new pronormoblasts. In parallel studies erythropoietin increased the cellular uptake of $\mathrm{C}^{14}$-glycine and $\mathrm{C}^{14}$-formate by nearly $100 \%$. Similar studies have been made on reticulocytes, on frozen or preincubated bone marrow, and on bone marrow cultures. These studies indicate that erythropoietin does not increase the iron uptake of individual cells, but they have not yet disclosed the exact site of action.

Studies on the Mechanism of Ethanol-induced Hypoglycemia. James B. Field* and Hibbard E. WilLIAMs, Bethesda, Md.

Hypoglycemia after alcohol ingestion has been attributed to nonethanol ingredients. Hypoglycemia has been repeatedly produced in one such subject and all of five normal controls by the following procedure. After 42 hours of fasting, two control blood sugars an hour apart were obtained and then 35 to $50 \mathrm{ml}$ of ethanol was given orally. Hypoglycemia developed within 1 hour and persisted for at least 3 hours, at which time glucagon did not increase blood sugar. Fasting without ethanol caused no hypoglycemia. Equal amounts of ethanol after an overnight fast did not produce hypoglycemia or impair glucagon-induced hyperglycemia. Plasma free fatty acids remained constant at an elevated level for the last 5 hours of a control 48-hour fast but rose progressively during the hypoglycemia after ethanol, indicating that hypoglycemia was not due to increased insulin. During the ethanol-induced hypoglycemia the blood glucose rise after $50 \mathrm{~g}$ fructose (given i.v. 2 hours after ethanol) was comparable with that during a control fast without alcohol, indicating unimpaired conversion of fructose to glucose. However, ethanol inhibited the conversion of fructose to glycogen as indicated by the small response to glucagon (given 1 hour after fructose) compared with the marked hyperglycemia after fructose and glucagon during the control fast. Similar inhibition of glycogen synthesis was demonstrated in vitro with liver slices from ethanol-treated rabbits. Perfusion of isolated rat liver (in collaboration with Dr. Glenn E. Mortimore), using an alcohol concentration similar to 
the studies in man, demonstrated normal conversion of fructose to glucose but marked inhibition of glycogen synthesis from fructose. These studies suggest that ethanol inhibition of glycogen synthesis may be primarily responsible for the observed hypoglycemia and that glycogen formation during gluconeogenesis may be a prerequisite for hepatic glucose release.

An Approach to the Prevention of Rh Hemolytic Disease. R. Finn and J. R. Krevans, Baltimore, Md. and C. A. Clarke, Liverpool, England (introduced by C. B. Thomas).

We have reported evidence suggesting that the low incidence of $\mathrm{Rh}$ hemolytic disease of the newborn is due to infrequent occurrence of transplacental hemorrhage of sufficient magnitude to induce sensitization. The detection of such hemorrhages in mothers after delivery, by the Betke technique, which demonstrates fetal red cells in the maternal circulation, enables one to pick out a small "high risk" group in whom sensitization is most likely to occur. The work of Levine, showing that $\mathrm{ABO}$-incompatible pregnancies interfered with $\mathrm{Rh}$ sensitization, raises the possibility that sensitization could be prevented if it were possible to remove $\mathrm{Rh}$ positive cells from the circulation of the "high risk" mothers. To test this hypothesis $\mathrm{Rh}$ negative males were given $5 \mathrm{ml}$ of $\mathrm{Cr}^{51}$-labeled $\mathrm{Rh}$ positive red cells and one-half hour later varying amounts of anti-D antibody were injected. Approximately $10 \%$ of controls who received only $R$ h positive cells developed anti-D. In the group who received 35 to $50 \mathrm{ml}$ of anti-D there was very rapid removal of $\mathrm{Rh}$ positive cells (half-life, 3 hours) and no immunization occurred. In the group receiving $10 \mathrm{ml}$ of anti-D, the $\mathrm{Rh}$ positive cell half-life was 24 hours, and there was a remarkable enhancement of immunization; $75 \%$ developed anti-D. These results suggest that immunization to the $\mathrm{Rh}$ antigen can be prevented if $\mathrm{Rh}$ positive cells are rapidly removed from the circulation. The mechanism by which immunization is enhanced by small doses of antibody seems to be related to accelerated removal at an intermediate rate.

The Relationship of Cations and ATP to Mitochondrial Swelling and Shrinkage. Sidney S. Fortney AND William S. Lynn,* Durham, N. C.

Mitochondrial swelling and shrinkage can be studied by 1) turbidity, 2) wet weight of pellet after centrifugation, and 3) urea- $\mathrm{C}^{14}$ space of pellet after centrifugation. By all of these methods it can be shown that swelling is associated with an increase in water content of the mitochondrial pellet. Swelling is also associated with an increase in ATPase activity in a linear fashion. Mitochondrial swelling occurs spontaneously in isotonic buffered media at $25^{\circ} \mathrm{C}$. This spontaneous swelling proceeds more rapidly in ionic media; e.g., $\mathrm{KCl}, \mathrm{NaCl}$, $\mathrm{LiCl}$, or $\mathrm{CsCl}$ than in sucrose of the same total osmotic strength. The rate of swelling induced by these ionic buffers has an inverse relationship to the hydrated radius of the cation. That is, mitochondria swell more rapidly in potassium and cesium than in lithium or sodium. ATP can increase the rate of swelling in sucrose at $25^{\circ}$, but this is completely blocked by EDTA at $10^{-4} \mathrm{M}$. However, swelling induced by ionic buffer-e.g., $\mathrm{Na}^{+}$, $\mathrm{K}^{+}$or $\mathrm{Li}^{+}$-is partially inhibited by ATP or EDTA, but completely inhibited when both are added together. In mitochondria partially depleted of calcium by washing with lithium, and then reincubated in a sucrose sodium buffer, we have found a response to ATP on the movement of cations. Under these conditions ATP liberates the more tightly bound calcium, and the efflux of lithium into the buffer is increased. Simultaneously, the influx of sodium from the buffer is inhibited by ATP as measured both by flame photometry and $\mathrm{Na}^{22}$. Thus, shrinkage induced by ATP is accompanied by a loss of intramitochondrial cations and a decreased permeability to extramitochondrial sodium.

Primary Hyperoxaluria: A Defect in Glyoxylate Metabolism. Elizabeth W. Frederick, Mitchell T. Rabkin ANd Lloyd 'H. Smith, JR.,* Boston, Mass.

Primary hyperoxaluria is a rare genetic disorder characterized biochemically by urinary excretion of excessive oxalate. The clinical manifestations include nephrolithiasis, nephrocalcinosis, and often a generalized deposition of calcium oxalate, termed oxalosis. The disease usually progresses to early death in renal failure. Previous studies have established that oxalate is not metabolized in man. The presumed genetically determined metabolic defect must therefore precede oxalate synthesis. The metabolism of glyoxylate, the only compound demonstrated to be an immediate precursor of oxalate, has not been previously studied in man. Two siblings with juvenile primary hyperoxaluria demonstrated a marked block in the metabolism of intravenously administered carboxyl-labeled glyoxylate- $\mathrm{C}^{14}$, excreting respiratory $\mathrm{CO}_{2}-\mathrm{C}^{14}$ at a rate only $20 \%$ of that found in control subjects. The rate of glyoxylate- $\mathrm{C}^{14}$ metabolism in the parents of the patients was approximately $65 \%$ of control values, suggesting a heterozygous defect. The patients with hyperoxaluria excreted 78 to $130 \mathrm{mg}$ oxalate per day, 3- to 5-fold normal levels. Normal daily urinary glycolate excretion, measured by an isotope dilution technique, averaged $43 \mathrm{mg}$. Both patients with hyperoxaluria also exhibited persistent glycolic aciduria, averaging $137 \mathrm{mg}$ per day-an increase comparable with that of oxalate. Preparations of leukocytes from patients and control subjects synthesized glycolate and oxalate from glyoxylate- $\mathrm{C}^{14}$ at similar rates. The oxidation of glyoxylate to oxalate produces a useless and potentially harmful compound. The development of an effective inhibitor of this enzymatic reaction would furnish a logical therapy for hyperoxaluria. In a survey of structural analogs in vitro, sodium hydroxymethanesulfonate was found to be a potent competitive inhibitor of oxalate synthesis from glyoxylate. The potential effectiveness of this inhibitor in vivo is currently being explored. 
Studies on the Pathogenesis and Clinical Features of "Alcoholic Hypoglycemia." N. FreIN KeL,* D. L. Singer, C. K. Silbert and J. B. Anderson, Boston, Mass.

In the past 2 years, admission to the Boston City Hospital of seven subjects with hypoglycemic coma and history of recent alcoholic ingestion afforded the opportunity to study this poorly defined entity first described by Brown and Harvey in 1941. Analysis of presenting features disclosed that coma: 1) occurred after prolonged imbibition without adequate diet; 2) was not restricted to chronic alcoholics; 3) was accompanied by hypothermia; and 4) was terminated within minutes after glucose administration. Liver functions and biopsies were normal to minimally abnormal; tests of thyroid, adrenal, and pituitary status (including methopyrapone) were normal. All subjects exhibited abnormalities in one or more of the following challenges to blood-sugar maintenance: insulin (i.v.); tolbutamide (i.v.) ; or fasting ( 72 hours). During prolonged observations on a metabolism ward, with excellent alimentation and total abstinence, the abnormalities persisted. In the three subjects in whom it was examined, i.v. or p.o. alcohol reproduced hypoglycemia after an overnight fast; more profound hypoglycemia was elicited after 72 hours of fasting. That pure ethanol, per se, constitutes a hypoglycemic agent was confirmed in normal subjects; however, 72 hours of prior fasting was necessary for such documentation. Manifest rise in peripheral glucose utilization and plasma insulin (immunoassay) did not accompany the hypoglycemia. The suggestion of direct effects upon hepatic gluconeogenesis has been confirmed by in vitro studies wherein ethanol reduced the production of glucose- $\mathrm{C}^{14}$ from pyruvate- $\mathrm{C}^{14}$ and alanine- $\mathrm{C}^{14}$ by liver slices concomitant with an increased formation of lactic acid- $\mathrm{C}^{14}$ (presumably due to the augmented DPNH from alcohol oxidation). The conjoint clinical and in vitro observations indicate that "alcoholic hypoglycemia" constitutes a distinct syndrome which is readily unmasked in subjects who are inordinately vulnerable to diversion of 3-carbon fragments from gluconeogenetic pathways.

Transfusion of Granulocytes from Donors with Chronic Myelocytic Leukemia to Leukopenic Recipients. E. J. Fretreich, E. E. Morse, W. Bronson and P. P. Carbone, Bethesda, Md. (introduced by James A. Shannon).

The technique of plasmapheresis has increased the availability of fresh platelets and granulocytes for transfusion studies. While sufficient quantities of platelets can be obtained from normal donors for control of hemorrhage, the relatively small numbers of circulating granulocytes preclude use of normal donors for granulocyte transfusion. However, patients with chronic myelocytic leukemia (CML) and white blood cell counts of 100,000 to 300,000 per $\mathrm{mm}^{8}$ can donate adequate numbers of granulocytes in relatively small plasma volumes. Five patients with CML donated $500 \mathrm{ml}$ of granulocyte-rich plasma as often as 5 days a week for 3 weeks. The median dose was $5 \times 10^{10}$ granulocytes per transfusion (range, $2 \times 10^{10}$ to $1 \times 10^{11}$ ). Fifty such transfusions were given to 19 leukopenic recipients, 17 of whom had acute leukemia. This resulted in a median 1-hour post-transfusion increment in circulating granulocytes of 1,000 per $\mathrm{mm}^{3}$ (range, 0 to 11,000 ). The median recovery in the circulation was $5 \%$ (range, 0 to 20 ) of the dose given. Circulating granulocytes returned to pretransfusion levels over the next 1 to 4 days (median, 2). With repeated daily treatment, granulocyte levels could be maintained above 1,000 per $\mathrm{mm}^{3}$. Transfusions have been repeated as often as 14 times for a single recipient. Thirty-two granulocyte transfusions were given during febrile episodes. In 14 of these, defervescence occurred within 12 hours. Ten patients with fever had positive blood cultures for pseudomonas organisms prior to transfusion. Nine had received colymycin or polymixin for 2 or more days before transfusion. In six of these patients fever returned to normal within 12 hours of transfusion, and they remained afebrile for more than 2 weeks. Transfusion of granulocytes from donors with CML has raised the circulating granulocyte levels of leukopenic recipients and has resulted in cure of pseudomonas septicemia.

Direct Evidence for the Participation of Endogenous Lipids in the Formation of Dietary Chylomicrons. Samuel J. Friedberg, Morton D. Bogdonoff, E. Harvey Estes, Jr., Paul B. Sher and Gitta Jackson, Durham, N. C. (introduced by David T. Smith).

Previously reported data have provided conflicting evidence concerning whether or not the fatty acid composition of dietary chylomicrons resembles that of the fed fat or assumes the pattern of the body fatty acids. The present investigation was designed to resolve this problem. Fasting male subjects were given a $100-\mathrm{g}$ fat load in the form of cream. Three hours later, at a time when alimentary lipemia was well established, albumin-bound radiopalmitate was injected i.v. Serial blood samples were analyzed for triglyceride activity and total triglyceride over the following 8 hours. Chylomicrons from separate aliquots of serum were isolated by ultracentrifugation. The supernatant chylomicrons obtained were washed three times by resuspending in saline and recentrifuged. The pooled infranatant solutions and washed chylomicrons were extracted and total radioactivity determined. The results indicate that radioactivity in total serum triglyceride appears in 30 minutes and reaches a peak in 2 hours. Samples of serum obtained 2 and 3.5 hours after injection of radiopalmitate contain 4 to $24 \%$ of the total triglyceride activity in the chylomicron fraction. In interpreting the results of these studies, passive exchange of the palmitic acid-1- $\mathrm{C}^{14}$ with serum triglycerides could be ruled out, because radioactivity in the triglyceride fraction was virtually absent during the 10 - to 15-minute period when radiopalmitate activity was maximal. It is concluded, therefore, that endogenously released free fatty acid (FFA), represented by the 
radiopalmitate, is in part incorporated as triglycerides into chylomicrons. This exchange could take place in the liver which takes up chylomicrons and converts FFA to triglyceride. Endogenously produced triglyceride might also exchange with alimentary chylomicrons in blood, intestinal lymphatics, or gastrointestinal tissues.

The $P_{\mathrm{CO}_{2}}$ of the Right and Left Side of the Circulation during $\mathrm{CO}_{2}$ Inhalations and Exercise. HermaN $\mathrm{F}$. Froeb ANd Byong MoK KIM, La Jolla, Calif. (introduced by Edmund L. Keeney).

To investigate the concept of a chemoreceptor on the right side of the circulation for the control of breathing, the following experiments were performed during cardiac catheterization in 11 patients. There were two with functional murmurs (C.H., P.S.) and the remaining had severe cardiopulmonary disease. While breathing through a pneumotachograph, nine patients inhaled $8 \% \mathrm{CO}_{2}$ and two $5 \% \mathrm{CO}_{2}$. Continuous recordings of respiratory peak flow and volume along with blood samples from pulmonary and brachial artery were obtained at 10 -second intervals for 2 minutes. Three of these patients were followed through recovery. Similar experiments were performed on six patients who exercised while breathing room air; two of three were followed through recovery. The results reveal that while the patients breathed $8 \%$ $\mathrm{CO}_{2}$, there were simultaneous rises in pulmonary and brachial arterial $\mathrm{P}_{\mathrm{CO}_{2}}$ with rising ventilation. In Subject C.H., who breathed $5 \% \mathrm{CO}_{2}$, there was no rise in brachial arterial $\mathrm{P}_{\mathrm{CO}_{2}}$ with rising ventilation, but a significant correlation with increasing pulmonary arterial $\mathrm{P}_{\mathrm{co}_{2} \text {. }}$ During recovery from $8 \% \mathrm{CO}_{2}$ in Subject P.S., decreasing ventilation followed the slow decline of the pulmonary arterial $\mathrm{P}_{\mathrm{CO}_{2}}$ over 80 seconds while brachial arterial $\mathrm{P}_{\mathrm{CO}_{2}}$ fell precipitously in 30 seconds. In patients with cardiopulmonary disease, the correlation of pulmonary arterial $\mathrm{P}_{\mathrm{CO}_{2}}$ and ventilatory changes was not clear-cut. In five subjects, during exercise, there was a linear correlation with increasing pulmonary arterial $\mathrm{P}_{\mathrm{CO}_{2}}$ to rising ventilation while brachial arterial $\mathrm{P}_{\mathrm{CO}_{2}}$ fell. One subject with tetralogy and Blalock operation was the only case in whom ventilation followed the brachial arterial $\mathrm{P}_{\mathrm{CO}_{2}}$. These results support the hypothesis of a chemoreceptor on the right side of the circulation.

Properties of Univalent 3.5S Fragments of $7 S$ Agglutinating Antibodies. Hugh Fudenberg and A. NisonofF, San Francisco, Calif. and Urbana, Ill. (introduced by Ernest Jawetz).

Purified 7S rabbit antibody to human erythrocytes and to human $7 \mathrm{~S} \gamma$-globulin was converted to $5 \mathrm{~S}$ and $3.5 \mathrm{~S}$ fragments with pepsin and papain, respectively. The 5S and $3.5 \mathrm{~S}$ fragments have been demonstrated previously to be bivalent and univalent, respectively. The 7S agglutinating antibody against human erythrocytes possessed apparent anti-H specificity. Its $5 \mathrm{~S}$ derivative retained full agglutinating activity, but the 3.5S fragments lacked agglutinating activity. However, combination of the $3.5 \mathrm{~S}$ fragments with the erythrocyte antigens was demonstrable by agglutination upon the addition of chicken or goat antisera prepared against the fragments of rabbit $\gamma$-globulin. The 3.5S univalent subunits of anti- $\mathrm{H}$ failed to agglutinate appropriate red cells by albumin, papain, ficin, or trypsin techniques, in contrast to the behavior of socalled "univalent" 7S incomplete antibodies such as anti- $R h_{0}$. Similar results were obtained with the rabbit antihuman $\gamma$-globulin antibodies, using $\mathrm{Rh}_{0}$ cells "coated" with incomplete anti- $\mathrm{Rh}_{0}$ as indicators. The $5 \mathrm{~S}$ derivatives of the antiglobulin serum possessed full agglutinating activity for these coated cells, whereas the $3.5 \mathrm{~S}$ fragments failed to agglutinate the coated cells. Combination of the $3.5 \mathrm{~S}$ fragments with the antigen (human $\gamma$-globulin) was again demonstrable by use of goat or chicken antibodies specific for the $3.5 \mathrm{~S}$ fragments. Treatment of the $5 \mathrm{~S}$ materials with sulfhydryl reagents resulted in production of $3.5 \mathrm{~S}$ fragments with serologic properties identical with those of the $3.5 \mathrm{~S}$ materials resulting from papain treatment of the intact $7 \mathrm{~S}$ antibody. These results indicate that the carbohydrate-rich "piece III" of rabbit antibody plays no role in agglutination reactions and that the univalent fragments possess sensitizing but not agglutinating activity.

Blood Angiotensin Levels in Normal Subjects and Hypertensive Patients. J. Genest, P. Biron, M. Chrétien, R. Boucher and E. Korw, Montreal, Canada (introduced by J. S. L. Browne).

Using the method of Boucher, Biron and Genest based partly on the procedure of Palladini and co-workers, 105 determinations of angiotensin blood level were done, mostly on brachial arterial blood, from 84 normal subjects and hypertensive patients. The average concentration in 33 normal subjects (35 determinations) was 10 $\mathrm{m} \mu \mathrm{g}$ per $100 \mathrm{ml}$ of blood (range, 0 to 55 ). Thirty-nine angiotensin determinations in 28 patients with essential hypertension showed an average concentration of 76 $\mathrm{m} \mu \mathrm{g}$ per $100 \mathrm{ml}$ (range, 0 to 820 ) ; 25 of these 39 determinations were within normal range. One patient with essential hypertension and without renal arterial disease had a concentration of $90 \mathrm{~m} \mu \mathrm{g}$ per $100 \mathrm{ml}$ in his renal venous blood. Two patients with hypertension associated with bilateral parenchymatous renal disease had angiotensin arterial levels of 0 and $100 \mathrm{~m} \mu \mathrm{g}$ per 100 $\mathrm{ml}$. Seven patients with hypertension associated with unilateral renal arterial stenosis had arterial levels of $265,80,110,10,35,26$, and 25 ; and renal venous levels of $100,0,130,30,0,280$, and $20 \mathrm{~m} \mu \mathrm{g}$ per $100 \mathrm{ml}$. Eight determinations made in 7 patients with malignant hypertension gave an average concentration of $18 \mathrm{~m} \mu \mathrm{g}$ per $100 \mathrm{ml}$ (range, 0 to 70 ). Seven determinations were below $35 \mathrm{~m} \mu \mathrm{g}$ per $100 \mathrm{ml}$. Blood angiotensin levels were normal in 5 patients with proven primary aldosteronism. No correlation could be found between the angiotensin blood level and the volume of the blood sample, the diastolic blood pressure level at the time of the sampling or the simultaneously determined urinary aldosterone. Although angiotensin may appear to play 
a role in the genesis of hypertension in a few patients, these studies strongly suggest that in the majority of hypertensive patients, whether of the essential, renal (parenchymatous) or malignant type, angiotensin is not involved in the maintenance of hypertension.

Acute Hemoglobimuric Renal Failure without Ischemia. Martin Goldberg, Philadelphia, Pa. (introduced by J. Russell Elkinton).

It has been difficult to assess the specific effects of hemolyzed red blood cells ( $\mathrm{RBC}$ ) on renal hemodynamics based on previous work because: 1) production of experimental acute renal failure due to hemolyzed cells, hemoglobin, or methemoglobin was associated with severe dehydration or hypotension; 2) studies of renal blood flow under these conditions involved clearance or direct Fick-para-aminohippurate (PAH) methods difficult to interpret at low urine flows (V). To circumvent these difficulties, moderate quantities of homologous hemolyzed $\mathrm{RBC}(0.2$ to $0.3 \mathrm{~g} \mathrm{Hb} / \mathrm{kg})$ were infused into the aorta above the renal arteries in 12 dogs. Renal blood flow (RBF) was estimated by an indirect Fick method using krypton $^{85}$ not dependent on urine collection. The infusion of hemolysate resulted in acute reductions in V (50 to $95 \%)$, creatinine clearance $\left(\mathrm{C}_{\mathrm{C}_{r}} ; 6\right.$ to $\left.99 \%\right)$, and radioiodinated hippuran clearance $\left(\mathrm{C}_{\mathrm{H} 1 \mathrm{pp}} ; 16\right.$ to $\left.90 \%\right)$. The fractional changes in $\mathrm{C}_{\mathrm{c}_{r}}$ and $\mathrm{V}$ were related to $\mathrm{V}_{\text {control }}$ such that the lower the $\mathrm{V}_{\text {control }}$ the greater the decrease in both $\mathrm{C}_{\mathrm{Cr}} \quad(\mathrm{r}=0.88, \mathrm{p}<0.001)$ and $\mathrm{V}(\mathrm{r}=0.73, \mathrm{p}<0.02)$. Despite these changes, $\mathrm{RBF}$ and arteriorenal $\mathrm{O}_{2}$ did not change after the infusion (control $\overline{\mathrm{RBF}}=296 \mathrm{ml} / 100 \mathrm{~g} / \mathrm{min}, \mathrm{SD} \pm 14.3$; postinfusion $\overline{\mathrm{RBF}}=329 \mathrm{ml} / 100 \mathrm{~g} / \mathrm{min}, \mathrm{SD} \pm 29.6$ ). In five animals, mean changes in renal extraction ratios $(E)$ of hippuran and creatinine were $<10 \%$ despite significant decreases in $\mathrm{V}, \mathrm{C}_{\mathrm{Cr}}$, and $\mathrm{C}_{\mathrm{H} / \mathrm{pp}}$; in three of these the mean change in $E_{P A B}$ was 0 . In one experiment a $50 \%$ reduction in $E_{H i p p}$ and $E_{C r}$ occurred. In three animals decreases in $\mathrm{U} / \mathrm{P} \mathrm{Cr}$ and $\mathrm{U} / \mathrm{P}$ osmolality were observed, but in the remainder, increases in these ratios followed the infusion. Histologically, tubular damage in varying degree was present in each animal. The data suggest that blood pigment excretion may be associated with acute decreases in urine flow and clearances not related to renal ischemia. Intratubular obstruction and, in some instances, concomitant toxic tubular damage can explain these observations.

Mechanisms of Renal Acidosis. H. C. Gonrck, M. E. Rubini and M. H. Maxwell, Los Angeles, Calif. (introduced by M. I. Grossman).

The inability of patients with chronic renal disease to excrete hydrogen ion may be due to: 1) diminished $\mathrm{H}^{+}$ production by the kidney, 2) a limitation in available proton carriers in the urine (ammonia or phosphate buffer), or 3) deficient ionic exchange with fixed cation $\left(\mathrm{Na}^{+} \rightleftharpoons \mathrm{H}^{+}\right)$. Acid excretion was studied as follows in a group of patients with various types of renal disease: 1) total acid excretion was measured after a standardized oral ammonium chloride load, 2) the ability to increase titratable acid excretion was measured during isotonic neutral phosphate infusions, and 3 ) the ability to exchange $\mathrm{Na}^{+}$for $\mathrm{H}^{+}$was measured by infusing sodium sulfate under conditions of maximal sodium-retaining stimuli. An approximately equal reduction in excretion of titratable acid and ammonium was demonstrated during ammonium chloride loading. No limitation was seen in titratable acid excretion when neutral phosphate was infused, and the slope of titratable acid vs phosphate was similar to that of normal subjects. Although blood $\mathrm{pH}$ was unchanged during sodium sulfate infusion, the drop in urinary $\mathrm{pH}$ was similar to that evoked by ammonium chloride and accounted for the increase in titratable acid excretion. However, ammonium excretion relative to $\mathrm{pH}$ was invariably less than normal. Despite reduced $\mathrm{H}^{+}$ excretion in exchange for $\mathrm{Na}^{+}$, some patients showed a normal $\mathrm{Na}^{+}-\mathrm{K}^{+}$exchange, implying intact distal sodium reabsorption and exchange. The data indicate that: 1) there is no intrinsic limitation in $\mathrm{H}^{+}$production when sufficient buffer is provided, 2) the distal ion exchange mechanisms may be normal but ammonia production faulty, and 3) acidosis in renal disease primarily reflects a decreased ability to provide urinary proton carriers. Parallel studies in renal tubular acidosis (congenital and acquired) indicate that this disease is exceptional in that a true defect in hydrogen ion production may exist.

\section{Role of the Thymus in Development of Immunity. Robert A. Good,* Carlos Martinez, Olga K. Archer and Ben W. Papermaster, Minneapolis, Minn.}

Although the thymus has been subjected to extensive study, its role in the body economy has not been elucidated. The observation that an abnormality of the thymus (benign thymoma) occurs frequently in patients with acquired agammaglobulinemia suggested a role for this organ in immunologic reaction. Removal of the thymus in adult rabbits did not alter immunologic reactivity. Glick and co-workers showed that removal of the bursa of Fabricius at an early age interfered with the immunologic development of chickens, and Mueller and co-workers showed more profound depression of immunologic potential in chickens lacking the bursa at hatching. We postulated that thymectomy in neonatal animals might interfere with the normal development of immunologic competence. Studies in our laboratory, using rabbits, rats, and inbred mice, have shown that the thymus is indeed essential to normal immunologic development. Animals thymectomized in the immediate neonatal period have a morphologically defective lymphoid system, deficient homograft and tumor immunity, and feeble capacity for formation of precipitating and phageneutralizing antibody. It is postulated that the thymus is involved in the centrifugal distribution of immunologically competent cells which are ultimately involved in the several forms of immunologic reactivity. 
Generation of Reduced Pyridine Nucleotides in the Livers of Normal and Alloxan-diabetic Rats. EDwin E. GoRDoN, New York, N. Y. (introduced by Irving M. London).

A major defect in the metabolism of the diabetic organism is the markedly diminished ability of the liver to synthesize fatty acids. In the present experiments an attempt was made to assess directly the hypothesis that diminished generation of reduced pyridine nucleotides might be a significant rate-limiting factor for fatty acid synthesis in the liver of the diabetic rat. Nicotinamide-7$\mathrm{C}^{14}$, introduced intraperitoneally, labels the DPN and TPN of liver. The reduced forms of the pyridine nucleotides are generated from the corresponding oxidized forms in the course of pyridine nucleotide-linked enzymatic reactions. The specific radioactivity of each of the four forms as a function of time after administration of nicotinamide- $\mathrm{C}^{14}$ provides data relating to the rates of equilibration of the pyridine nucleotides. The specific activity of hepatic DPN increases progressively, while that of TPN does not change appreciably between 2.5 and 20 minutes after administration of nicotinamide- $\mathrm{C}^{14}$. The ratio of the specific activity of DPNH to DPN reaches 0.5 within 2.5 to 5 minutes and approaches unity at approximately 15 minutes in the livers of both normal and diabetic rats. In normal liver, equilibration of TPNH with TPN does not occur until after 20 minutes, and the ratio reaches 0.5 between 10 and 15 minutes. The rate of equilibration of the triphosphopyridine nucleotides in the diabetic liver is the same as, or more rapid than, that found in the normal. Since the corresponding pool sizns of each of the four pyridine nucleotides are essentially equivalent in normal and diabetic rat livers, the equilibration data are a function of the rate of generation of reduced pyridine nucleotides. These data therefore, suggest that the rate of generation of DPNH and TPNH is independent of the capacity of alloxan-diabetic rat liver to synthesize fatty acids. The generation of reduced pyridine nucleotides in the major intracellular compartments of normal and diabetic liver is currently under study.

Dynamic Dilution Studies of Substances Passing through the Portal Circulation in the Liver. CARL A. Goresky, Montreal, Canada (introduced by Douglas G. Cameron).

Labeled red cells, serum albumin, sucrose, inulin, sodium, and water were given by a single rapid injection into the portal vein in the dog. Their subsequent concentrations in the hepatic venous blood were sampled serially. None of these substances was appreciably retained in the liver on one circulation, but the outflow pattern of each of the substances was modified relative to the red cell pattern, showing a diminution in peak concentration and delayed transit time. A substance, such as water, that might be expected to enter the total organ water, showed a greater peak depression and prolongation of transit than did substances like sucrose that one might expect were excluded from the intracellular water. Theoretical considerations of flow through liver sinusoids of substances that exchange into an extravascular space led to a quantitative mathematical treatment in terms of two parameters, the extravascular volume of distribution and the effective sinusoidal blood volume (i.e., the volume in the vessels across which exchange occurs). These parameters are calculated from a group of indicator dilution curves. The two calculated parameters accurately describe the changes in each curve induced by extravascular exchange. The volume calculated for water agrees with the steady-state value obtained from the extirpated organ. There is a rapid extravascular exchange of plasma albumin into a space about equal to the intravascular albumin space. This fact probably accounts for the apparent low "whole organ" hematocrit reported for this organ. A quantitative method has been derived to describe the passage of diffusible indicator substances through the hepatic sinusoids in terms of two previously inaccessible parameters, 1) sinusoidal blood volume, and 2) the volume of distribution within the organ. This method may facilitate the calculation of effective capillary blood volume and of the volume of distribution of diffusible substances in other organs as well.

A Re-evaluation of the Mechanism of Endotoxin Tolerance. Sheldon E. Greisman* and Frank A. Carozza, Jr., Baltimore, Md.

After reticuloendothelial system (RES) blockade, animals tolerant to bacterial endotoxin exhibit febrile reactions to endotoxin that are comparable with normal animals. These observations initiated the hypothesis that endotoxin tolerance is mediated primarily by the RES. It should be emphasized, however, that tolerant animals, deprived of a normally functioning $\mathrm{RES}$, were being compared with normal intact animals. The fact that both groups react comparably to endotoxin actually suggests that tolerant animals with blockaded RES possess a mechanism capable of inhibiting endotoxin in vivo about as effectively as does the intact RES of normal animals. To verify this concept, rabbits were rendered tolerant to $2.0 \mu \mathrm{g}$ Escherichia coli endotoxin. These animals and normal controls were then given Thorotrast $(3 \mathrm{ml} / \mathrm{kg}$ i.v.) and 15 hours later $2.0 \mu \mathrm{g} \mathrm{E}$. coli endotoxin. The control RES-blockaded animals exhibited significantly and markedly greater fever indices than did the tolerant blockaded group. To exclude the possibility that these reactions were attributable to resistance of tolerant animals to RES blockade, normal animals were given Thorotrast intravenously $(3 \mathrm{ml} / \mathrm{kg})$; 3 hours later $10 \mathrm{ml}$ per $\mathrm{kg}$ of pyrogen-free, fresh, heparinized plasma was administered intravenously from donor animals tolerant to $2.0 \mu \mathrm{g}$ per $\mathrm{kg} E$. coli endotoxin. Control blockaded animals received comparable quantities of fresh normal plasma. Sixty minutes after plasma transfer, all animals received Salmonella typhosa endotoxin intravenously $(0.05 \mu \mathrm{g} /$ $\mathrm{kg}$ ). RES-blockaded animals given tolerant plasma exhibited tolerant-shaped fever curves and significantly lower fever indices than did blockaded animals given 
control plasma. These studies indicate that when proper controls are employed, tolerance to endotoxin can be associated with the appearance of a potent, nonimmunologically specific, humoral inhibitor of endotoxin that acts effectively in vivo despite RES blockade. Such findings suggest that humoral inhibitors play a more important role in mediating the tolerant state than has been previously suspected.

Isolation of d-Tubocurarine-binding Protein from Skeletal Muscle. David Grob,* Tatsuji Namba, Nathan A. Solomon and Daniel S. Feldman, Brooklyn, N. Y.

In an effort to identify the receptor in skeletal muscle that combines with the neuromuscular transmitter, acetylcholine, studies have been carried out on the combination of muscle constituents with $d$-tubocurarine, which blocks transmission by competing with acetylcholine for the receptor. $d$-Tubocurarine-binding substance was identified in normal human muscle by $\mathrm{C}^{\mathbf{1 4}}$-labeled dimethyl- $d$ tubocurarine and concentrated by repeated precipitation with $d$-tubocurarine. The binding substance is a ribonuceloprotein, of which $60 \%$ is RNA and $40 \%$ protein. It gave characteristic ultraviolet and infrared absorption spectra. The amino acid composition of the protein was determined and the presence of quanine, adenine, cystosine, uracil, and pentose was proven. The binding by the protein of $d$-tubocurarine or dimethyl- $d$-tubocurarine was inhibited by compounds that antagonize the neuromuscular blocking action of these agents, including acetylcholine, choline, succinylcholine, and decamethonium. The addition of acetylcholine to the protein produced a prompt change in the infrared absorption spectrum in aqueous medium, and a prompt increase in the proportion of hydrogen atoms which were rapidly exchangeable with isotopic hydrogen. These changes indicate that acteylcholine may alter the structure of the protein by rupturing hydrogen bonds, increasing water binding, or both. $d$-Tubocurarine did not have this effect. The reactions of $d$-tubocurarine and acetylcholine with $d$-tubocurarinebinding muscle protein were not specific for this protein but also occurred with casein and thrombin, although not with albumin, globulins, hemoglobin, gelatin, or elastin. $d$-Tubocurarine also reacted with RNA, DNA, chondroitin sulfuric acid, and polyserine. Preliminary studies have indicated some differences between the $d$-tubocurarine-binding protein isolated from the skeletal muscle of normal subjects and patients with myasthenia gravis, cardiac muscle, and muscle of other species.

Stimulation of Radiosulfate Incorporation into Guinea Pig Harderian Gland by Thyroid-stimulating Hormone (TSH) and Exophthalmic Serum. Heskel M. HADDAD, St. Louis, Mo. (introduced by David M. Kipnis).

The 24-hour uptake of $\mathrm{S}^{35} \mathrm{O}_{4}$ by guinea pig orbital tissues and the Harderian gland in particular can be stimulated by TSH $(1.0 \mathrm{U} /$ day $\times 2)$. TSH stimulation of Harderian gland sulfation can be made more sensitive by injecting $\mathrm{S}^{35} \mathrm{O}_{4}$ intraperitoneally 72 hours before killing the animals, during which time $\mathrm{TSH}$ is administered in three daily doses. An increase of 24 to $56 \%$ in Harderian gland sulfation was obtained with $0.1 \mathrm{U}$ of TSH per day $\times 3$. The effect of TSH was not reproduced by large doses of growth hormone, prolactin, ACTH, or thyroid hormones. The sera of seven patients with progressive endocrine exophthalmos and four with inactive exophthalmos were screened for TSH sulfationlike activity, injecting 0.1 to $0.2 \mathrm{ml}$ of serum intraperitoneally daily for 3 days. The sera of seven patients with progressive exophthalmos produced an 18 to $44 \%$ increase in Harderian gland radiosulfate incorporation. Of the four patients with inactive exophthalmos, two sera caused no change and two induced an $11 \%$ rise in Harderian gland $\mathrm{S}^{35} \mathrm{O}_{4}$ uptake. The uptake of $\mathrm{S}^{35} \mathrm{O}_{4}$ by the thyroid gland was persistently stimulated by TSH (average increase, $34 \%$ ). Sera of nine patients, who were currently euthyroid (four with inactive and five with progressive exophthalmos) caused no significant change in thyroid $\mathrm{S}^{25} \mathrm{O}_{4}$ uptake. The sera of two thyrotoxic patients with progressive exophthalmos stimulated thyroid $\mathrm{S}^{35} \mathrm{O}_{4}$ incorporation by 27 and $42 \%$. These findings suggest that a sulfating factor exists in exophthalmic sera which stimulates the Harderian gland but not the thyroid tissue of the guinea pig.

Unusual Properties of Synovial Fluid Hyaluronate in Rheumatoid Arthritis. David Hamerman* AND John Sandson, New York, N. Y.

Hyaluronate was isolated from synovial fluids obtained from normal joints and from inflamed joints of patients with rheumatoid arthritis. The fluids were passed through a column of hydroxylapatite and IRC-50 resin, and the hyaluronate isolated by ultrafiltration on Millipore filters $(0.1 \mu$ pore diameter $)$. Normal products contained hyaluronate and about $2 \%$ protein (NHP) with a high content of alanine, glycine, serine, and glutamic acid. Rheumatoid hyaluronate (RHP) consistently contained more protein (5 to $7 \%$ ) with a higher content of basic amino acids. To show that protein and hyaluronate were firmly bound, the products were labeled with iodine $^{\mathbf{1 3 1}}$ and subjected to zone electrophoresis. Hyaluronate was located by analysis for hexuronic acid, protein by radioactivity. Both NHP and RHP migrated toward the anode as a single component over a $\mathrm{pH}$ range of 6.4 to 10.5 . At $\mathrm{pH} 4.5(0.075 \mathrm{M}$ acetate) a striking difference was noted: NHP moved toward the anode, RHP remained at the origin. The immobility of RHP was not reversed by $6 \mathrm{M}$ urea. Upon deploymerization of RHP by cysteine or ascorbic acid, RHP migrated as a single component toward the anode at $\mathrm{pH}$ 4.5. After digestion of the protein of RHP with trypsin, hyaluronate migrated toward the anode at $\mathrm{pH} 4.5$. Some samples of RHP $(1 \mathrm{mg} / \mathrm{ml}$ became "gel-like" after dialysis in acetate buffer ( $\mathrm{pH} 4.5)$ and would not flow in Ostwald viscometers $\left(25^{\circ} \mathrm{C}\right)$. Dialysis at $\mathrm{pH} 7.2$ restored these samples to a free-flowing state. The gel-like state at $\mathrm{pH}$ 4.5 , which could account for electrophoretic immobility, appeared to be due to aggregation of RHP, since these 
samples were retained on $5 \mu$ Millipore filters that permitted rapid filtration of NHP. Neither the electrophoretic immobility nor gel-like state of RHP at pH 4.5 could be demonstrated with NHP even when NHP was: 1) isolated from normal synovial fluid with added rheumatoid serum, or 2) mixed with the basic protein lysozyme. The unusual properties of $\mathrm{RHP}$ at low $\mathrm{pH}$ suggest that its structure differs from that of NHP.

Clinical Syndromes with Elevated Blood Histamine and Serotonin. Bernard J. Haverback, Eugene T. BaldRIDGe And Barbara Dyce, Los Angeles, Calif. (introduced by Clayton G. Loosli).

Specific, highly sensitive spectrophotofluorometric methods for histamine and serotonin analyses permit precise studies of these amines. The purposes of this study are 1) to present three diverse clinical syndromes in which whole blood histamine and platelet serotonin levels were significantly elevated and 2) to determine interrelationships of histamine and serotonin in the brain, mast cell, and gastrointestinal tract of the rat. The means of many blood histamine levels in eight patients with the malignant carcinoid syndrome were $0.12,0.13,0.18,0.04,0.06$, 0.09 , and $0.27 \mu \mathrm{g}$ per $\mathrm{ml}$ (normal, 0.02 to 0.08 ). All had elevated blood serotonin levels. The histamine levels in carcinoid tumor from four of these subjects were not elevated $(0.4,2.2,0.2$, and $12.5 \mu \mathrm{g} / \mathrm{g})$. Two subjects with transient flushes, abdominal pain, nausea, diarrhea, and no objective evidence of carcinoid tumor had mean values of platelet serotonin of 1.5 and $0.7 \mu \mathrm{g}$ per $\mathrm{mg}$ of platelet protein (normal 0.2 to 0.6 ) and of blood histamine of 0.15 and $0.21 \mu \mathrm{g}$ per $\mathrm{ml}$. One subject who attempted suicide by ingestion of a large amount of a potent monoamine oxidase inhibitor (trans-DL-2-phenylcyclopropylamine) which produced coma, extensor rigidity, tremor, hyperthermia, and a striking facial flush had a mean blood serotonin level of $1.75 \mu \mathrm{g}$ per $\mathrm{mg}$ and a mean blood histamine level of $0.10 \mu \mathrm{g}$ per $\mathrm{ml}$. Because of these findings, interrelationships of tissue histamine and serotonin were studied. In the rat it was shown that 1) serotonin or 5-hydroxytryptophan produced no change in tissue histamine; 2) reserpine depleted bound tissue histamine as well as bound tissue serotonin; 3) $\alpha$-methyl histidine lowered gastric mucosal histamine but did not decrease serotonin. It is clear that certain substances affect both serotonin and histamine, and that in the clinical conditions described herein, histamine as well as serotonin levels in the blood were elevated.

Reduction of Serum Cholesterol in Man by an Oral Androsterone Preparation. Leon Hellman,* Barnett Zumoff, Gerald Kessler, R. S. Rosenfeld and T. F. Gallagher, New York, N. Y.

The demonstration of the hypocholesterolemic properties of androsterone raised the possibility that this normal steroid metabolite might have a role in the pathogenesis and treatment of atherosclerosis. Although the hypocholesterolemic effect has been amply confirmed, evaluation of the clinical utility of long-term androsterone therapy has lagged, since it is ineffective when given orally and parenteral administration may be accompanied by local discomfort and occasional fever. It has been found that androsterone, when administered orally together with ethyl $p$-chlorophenoxyisobutyrate (Imperial Chemical Industries), produces a hypocholesterolemic response indistinguishable from that of the parenteral steroid, but free of the untoward effects : 1) 20 to $30 \mathrm{mg}$ androsterone together with 1 to $1.5 \mathrm{~g} p$-chlorophenoxyisobutyrate was given daily by mouth for periods up to 4 months to 13 hypercholesterolemic patients. Ten patients showed a $22 \%$ fall $(\mathrm{p}<0.01)$ in serum cholesterol from an initial mean value of $397 \mathrm{mg}$ per $100 \mathrm{ml}$. Free and ester cholesterol fell proportionately. 2) There was a concomitant $28 \%$ fall $(\mathrm{p}<0.01)$ in serum alkaline phosphatase. There was no change in the usual liver function tests. 3) Desmosterol could not be identified by vapor-phase chromatography in the sera of three subjects who had significant decreases in cholesterol levels. 4) The androsterone was absorbed and then excreted in the urine as a conjugated 17-ketosteroid. 5) No virilization, feminization, or changes in libido were observed. Since this combination is well tolerated and no toxicity has been observed thus far, the exploration of the longterm effects of hypocholesterolemic therapy with androsterone is now feasible.

On the Presence and Character of the Increased Ability of the Addisonian Patient to Taste Salt. R. I. HenKin, J. R. Gill, JR., F. C. Bartter * and D. H. SoloMoN, Bethesda, Md. and Los Angeles, Calif.

Adrenalectomized rats, unlike normal rats, can detect $\mathrm{NaCl}$ in very dilute solutions. In the present study the taste threshold to $\mathrm{NaCl}$ has been determined in patients with and without adrenal insufficiency. The subject was required to choose the salt solution from a series of three fluids, two of which were distilled water. Eleven different concentrations of $\mathrm{NaCl}$ were used, with a varied order of presentation. Threshold was measured as the lowest concentration of $\mathrm{NaCl}$ that could consistently be distinguished from distilled water. In 16 normal subjects and 75 patients without evidence of disease of the adrenal glands, the median detection threshold for $\mathrm{NaCl}$ was $12 \mathrm{mM}$ (range, 6 to $60 \mathrm{mM}$ ). In 12 patients with adrenal insufficiency the median detection threshold was markedly decreased to $0.4 \mathrm{mM}$ (range 0.1 to $2.5 \mathrm{mM}$ ). Treatment with deoxycorticosterone $(20 \mathrm{mg} /$ day $)$ alone did not alter the taste threshold despite a gain in weight and return of serum sodium concentration to normal. Treatment with carbohydrate-active steroids (prednisolone) raised the median detection threshold to the prior normal level of $12 \mathrm{mM}$ within 18 to 48 hours; this occurred without change in body weight or in serum sodium. An increase in the sensitivity of salt-tasting to abnormal levels returned within 4 days, after treatment with carbohydrate-active steroids was discontinued. An increase in ability to taste $\mathrm{NaCl}$ in minimal concentrations thus appears to be a characteristic sign of adrenal 
cortical insufficiency. Ability to return salt-tasting sensitivity to lower, normal levels appears to be a specific property of carbohydrate-active steroids.

A "Messenger"-like RNA in Mammalian Cells. Howard H. Hiatt,* Boston, Mass.

Transfer of information from the genes to the proteinsynthesizing machinery of bacteria is now widely considered to be mediated by a fraction of ribonucleic acid termed "messenger" RNA. Its metabolic instability, the similarity of its purine and pyrimidine base ratios to those of the corresponding bacterial deoxyribonucleic acid, the heterogeneity of its molecular size, and its capacity to attach to the protein-forming particles within the cell are among its characteristics in accord with the messenger concept. To ascertain the applicability of this hypothesis to mammalian cells we are investigating in vivo and in vitro RNA and protein synthesis in normal and regenerating rat liver and in human blood cells. From rat liver nuclei we have isolated a labile fraction of RNA which has a 20 -fold higher specific activity than has the bulk of the nuclear RNA 10 to 40 minutes after in vivo administration of orotic acid- $\mathrm{C}^{14}$ or $\mathrm{P}^{32}$, but which, in contrast to the remaining nuclear and cytoplasmic RNA, is no longer labeled 18 hours after injection of isotope. We have shown by a sucrose gradient centrifugation technique that the rapidly labeled material has the heterogeneity of size that would be required of a fraction coding for the diverse cellular proteins. The base ratios of this fraction are much closer to those of rat liver DNA than are those of the cytoplasmic RNA or the bulk of the nuclear RNA. When exposed to liver microsomes, the particles known to synthesize protein, over $75 \%$ of this fraction becomes attached. Preliminary studies. indicate that nuclear RNA containing the labile material stimulates the incorporation of amino acids into protein in a cell-free protein-synthesizing system prepared from liver, but not in one prepared from bacteria. These findings are consistent with the presence in mammalian cells of an RNA analogous to bacterial messenger.

Diminished Biological Half-life of Angiotensin II in Hypertensive Plasma. Roger B. HickleR, David P. Lauler and George W. Thorn,* Boston, Mass.

Interest in the metabolism of angiotensin in hypertension has resulted in an application of the rat bioassay for the determination of the biological half-life of angiotensin II in human plasma. An in vitro admixture of angiotensin II and plasma is studied for the progressive loss of pressor activity by serial injection in a 250-g vagotomized rat, prepared with pentobarbital and pentolinium. The left jugular vein is intubated with a microsyringe containing a standard $(1 \mu \mathrm{g} / \mathrm{ml})$ solution of angiotensin II; the right jugular with a microsyringe containing a mixture of $1 \mathrm{ml}$ of the standard angiotensin solution and $1 \mathrm{ml}$ of plasma. A stop watch is started at the time of the admixture. Room tempera- ture is constant at $25 \pm 1^{\circ} \mathrm{C}$. The arterial pressure response to the injection of the standard vs standard plus plasma solutions is alternately recorded every 3 or 4 minutes. The dose-response curve for standard angiotensin is linear in the range employed. Thus, the progressive reduction in the pressure response for the plasma plus standard mixture (after correction for any variation in the standard response at each point during the assay) is equivalent to the rate of inactivation of angiotensin by the plasma. The regression curve indicates a first-order reaction from which the biological half-life is determined. Angiotensin in the plasma of 11 normotensive subjects so studied resulted in a mean biological half-life of 30.2 minutes $(\mathrm{SE} \pm 1.9 \mathrm{~min}$ ) as compared with a mean of $18.1( \pm 1.5 \mathrm{~min})$ in the plasma of 12 patients with primary hypertension $(\mathrm{p}<0.001)$. The mean for 8 patients with hypertension associated with chronic renal disease was $24.6( \pm 1.7$ $\min$ ) and was significantly greater than that for the group with primary hypertension $(p=0.01)$. The diagnostic implications and the possibility that these changes may represent an enzymatic adaptation to chronic angiotensinemia are being investigated. A similar trend reported by Wolf and co-workers, using a radiochemical technique, is acknowledged.

Initiation of the Action of Complement in a Human Autoimmune Hemolytic System, the Donath-Landsteiner Reaction. CARL F. HINz, JR.* AND ANNA Marie Mollner, Cleveland, Ohio.

Serum complement mediates the cytolytic reaction in immune hemolysis including the autoimmune DonathLandsteiner (D-L) reaction of paraoxysmal cold hemoglobinuria. The biphasic nature of the D-L reaction and the requirement for complement in both its initial cold and final warm phases, make it adaptable as a model system entirely of human origin for studying separately the sequential phases of complement action. Previous studies from this laboratory indicated that $\mathrm{C}^{\prime} 1$, the first component of complement, or C'1-esterase, the enzymatically active form derived from partially purified $C^{\prime} 1$, is required in the early phase of the D-L reaction, consistent with current concepts of the action of complement. However, Müller-Eberhard has recently demonstrated that a normal serum protein with characteristics of an $11 \mathrm{~S} \gamma$-globulin is required very early in the lysis of sheep cells. The current observations indicate that this $11 \mathrm{~S}$ factor initiates the D-L reaction and acts before $C^{\prime} 1$. The evidence indicates that the partially purified $11 \mathrm{~S}$ factor and serum lacking $11 \mathrm{~S}$ are individually inactive in completing the initial phase, but combination of the two restores activity. Sequential exposure of erythrocytes and antibody to the $11 \mathrm{~S}$ factor and then to serum lacking $11 \mathrm{~S}$ also completes the initial phase, but the reverse order does not, indicating that $11 \mathrm{~S}$ interacts before other components of complement. Subsequent to interaction with $11 \mathrm{~S}$, the cell-antibody complex can also interact with C'1esterase to complete the initial phase, but $C^{\prime} 1$-esterase 
cannot precede $11 \mathrm{~S}$. The activity of $11 \mathrm{~S}$ is not affected by either $\mathrm{Na}_{3} \mathrm{H}$-EDTA or serum inhibitor of $\mathrm{C}^{\prime} 1$ esterase, whereas both block the action of $\mathrm{C}^{\prime} 1$-esterase. Thus, the order of reaction of the components of complement in the D-L system is $11 \mathrm{~S}$ factor followed by $\mathrm{C}^{\prime} 1$ or $\mathrm{C}^{\prime} 1$-esterase in the initial cold phase, after which $C^{\prime} 4, C^{\prime} 2$ and $C^{\prime} 3$ cause lysis in the warm phase.

Blockage of D-Cycloserine Antibiosis by D-Alanine. Paul D. Hoeprich, Salt Lake City, Utah (introduced by M. M. Wintrobe).

While often clinically effective against several kinds of bacteria, D-cycloserine (the isomeric form of cycloserine produced by Streptomyces orchidaceus) is usually ineffective according to in vitro testing with conventional culture media. When 262 bacterial isolates were simultaneously tested for cycloserine susceptibility in two synthetic broth media (which differed only in the presence of DL-alanine), Escherichia coli, Salmonella sp., providence bacilli, enterococci and Staphylococcus aureus were significantly more often susceptible in the absence than in the presence of alanine. With $60 E$. coli isolates, susceptibility to benzylpenicillin, dihydrostreptomycin, neomycin, polymyxin B, chloramphenicol, tetracycline, sulfadiazine, and nitrofurantoin were simultaneously and identically assayed. Comparison of the in vitro effectiveness of these antibacterial agents at concentrations obtainable in the blood of humans receiving clinically practical dosages of these agents revealed that cycloserine was more often inhibitory to $E$. coli than either dihydrostreptomycin or tetracycline and was as effective as neomycin. These susceptibility data are in keeping with the clinical experience that cycloserine is a useful agent in treating $E$. coli infections. D-alanine is the isomeric form of alanine that blocks $D$-cycloserine antibiosis. Using a strain of $E$. coli, the quantitative aspects of D-alanine:D-cycloserine antagonism were studied. Cycloserine antibiosis was subverted to a degree directly proportional to the quantity of $\mathrm{D}$-alanine provided, up to the point of equimolarity. When the concentrations were equal, or when $D$-alanine was present in excess, cycloserine had no antibiotic effect. L-alanine is without effect on D-cycloserine antibiosis. Yet testing, by means of conventional culture media, usually fails to reveal significant cycloserine susceptibility. When L-alanine containing synthetic broth medium was subjected to heat, as in ordinary sterilization by autoclave ( $121^{\circ} \mathrm{C}$ for 15 minutes), it was rendered antagonistic to cycloserine antibiosis. The assumption that racemization of L-alanine had resulted with heating was borne out by direct analysis of the broth for D-alanine.

Studies of Lipid Transport in the Tissues. William HoLlander, Richard N. Kaplan and Russell P. Sherwin, Boston, Mass. (introduced by Robert W. Wilkins).

Extraction of nonatherosclerotic arterial tissue with petroleum ether yielded less than $5 \%$ of its contained cholesterol, whereas extraction of atherosclerotic tissue yielded from 26 to $72 \%$ of its cholesterol. Extraction of normal plasma or of prepared $\beta$-lipoprotein yielded less than $5 \%$ of its contained cholesterol, whereas plasma or $\beta$-lipoprotein to which free crystalline cholesterol had been added yielded all of the added cholesterol. These studies indicated that atherosclerotic tissue contains sizable amounts of unbound cholesterol as well as lipoprotein-bound cholesterol. The fate of unbound and bound lipid upon injection into living tissues was therefore studied. $\mathrm{C}^{14}$-labeled cholesterol and $\beta$-lipoprotein. labeled with $\mathrm{C}^{14}$-cholesterol, were injected intradermally into separate sites on the normal human forearm. Skin biopsies of the injection sites were obtained 3 to 7 days later. In the biopsies, over $90 \%$ of the $\mathrm{C}^{14}$-cholesterol radioactivity was recovered, whereas less than $10 \%$ of the lipoprotein radioactivity was recovered. Similarly, $\mathrm{I}^{181}$-labeled triolein remained at the injection site, whereas lipoprotein labeled with $\mathrm{I}^{131}$-triolein was removed within 5 days. Removal of radioactive lipoprotein from a lymphedematous limb was significantly slower than from the opposite normal limb, indicating that removal is predominantly via the lymphatics. Histological studies on skin biopsies obtained 1 week after the intradermal injection of nonisotopically labeled lipids revealed that neither $\beta$-lipoprotein nor triolein caused any local inflammation, whereas the injected unbound cholesterol caused a chronic inflammatory response. After injection into the femoral arterial wall of dogs, $\mathrm{C}^{14}$-cholesterol radioactivity was $90 \%$ recoverable at the injection site 5 days later, whereas lipoprotein labeled with $\mathrm{C}^{14}$-cholesterol had decreased to less than $5 \%$ of the amount injected.

Metabolism of Arterioles and Venules as Studied in the Cartesian Diver. Rufus O. How ARd, David W. Richardson, Morris H. Smith, Jr. and John L. Patterson, JR.,* Richmond, Va.

The metabolism of the physiologically most active portions of the vascular bed, the arterioles and venules, has had little attention. This report concerns application of the Cartesian diver technic to measurement of oxygen consumption by arterioles and smallest arteries (130 to $560 \mu$ in diameter), and of venules and smallest veins $(300$ to $900 \mu)$. Ninety-nine mesenteric arterioles and 76 venules from 46 hamsters were studied in Ringer's phosphate plus $200 \mathrm{mg} \%$ glucose as substrate. Results were similar whether the animal was anesthetized (pentobarbital) or decapitated unanesthetized. With $21 \%$ oxygen in the diver, oxygen consumption of arterioles with in vivo diameters less than $300 \mu$ averaged $0.9 \mu 1$ per $\mathrm{mg}$ per hour, double that of arterioles and small arteries with diameters greater than $500 \mu(0.4 \mu \mathrm{l} / \mathrm{mg} / \mathrm{hr}$; $\mathrm{p}<0.05)$. Metabolism of hamster venules averaged 0.5 $\mu 1$ per $\mathrm{mg}$ per hour, significantly less than that of the smallest arterioles, but comparable with that of the largest arterial tissue studied. There was no relation between venous size and metabolic rate. Both arterioles and venules were highly resistant to hypoxia. Reduction 
in oxygen tension in the gas above the substrate in four steps from 150 to $12 \mathrm{~mm} \mathrm{Hg}$ produced no change in metabolic rate of hamster arterioles, but at $5 \mathrm{~mm} \mathrm{Hg}$, arteriolar oxygen consumption was reduced to $60 \%$ of control. Venules were even more resistant to hypoxia, maintaining normal metabolic rate at 5 , although not at $2 \mathrm{~mm} \mathrm{Hg}$. Ten human vessels of both types from five normal mesoappendices showed lower metabolic rates than hamster vessels. Human arterioles, 130 to $350 \mu$ in diameter, consumed 0.35 ; and venules of 260 to $900 \mu$ consumed $0.25 \mu 1 \mathrm{O}_{2}$ per hour. The greater resistance to hypoxia of venules as compared with arterioles may reflect adaptation of metabolic processes to the lower oxygen tension (approximately $50 \mathrm{~mm} \mathrm{Hg}$ ) of the venous environment. Working vessels in vivo might be expected to show higher metabolic rates under normal oxygen tension, and depression of metabolism by lesser degrees of hypoxia.

Responses of Isolated Toad Bladder to Anisosmotic Bathing Media. John A. Isom, Mehmet N. Kalayci and John D. Crawford,* Boston, Mass.

Net sodium transport (short-circuit current) of toad bladder bathed in Ringer's solution is sharply reduced when the medium is made hypertonic $(380 \mathrm{mOsm} / \mathrm{kg})$ by addition of $\mathrm{NaCl}$, sucrose, or urea. Potential difference concomitantly falls and resistance rises. The effect is fully reversible. The influence of hypotonicity was studied in sodium-poor ( $55 \mathrm{mEq} / \mathrm{L}$ ) Ringer's solution. The same fluid made eutonic by addition of sucrose served as control medium and was shown to sustain satisfactory levels of sodium transport. The hypotonic fluid caused fully reversible, large, and abrupt rises in short-circuit current and potential difference with decline in resistance. Vasopressin provoked increments in shortcircuit current both in membranes at the peak of their response to hypotonicity as well as in those in which prior exposure to hypertonic media had reduced electrical activity, in some instances almost to nil. In both circumstances, the extent of vasopressin response was similar to that of the control bladders in eutonic Ringer's. Vasopressin also rendered the otherwise unresponsive mucosal surface reactive to changes in medium tonicity. Measurements of tissue water content indicate 5.7 and 2.2\% losses after 30 minutes' incubation in Ringer's made hypertonic, respectively, with sucrose and $\mathrm{NaCl}$; no change with urea; and a $7.9 \%$ increase after incubation in the hypotonic medium. Vasopressin failed to influence tissue hydration. Changes in bioelectric behavior induced by anisosmotic media thus failed to correlate with alterations in tissue water. They may relate to changes in mitochondrial size, such as are known to occur with tonicity change, and recently have been demonstrated in renal medullary mitochondria exposed to vasopressin. Regardless of mechanisms, the findings, especially should they represent a general cellular phenomenon, appear to have interesting implications to volume control in the whole animal.
Loss of Functional Activity of Platelets during Storage. Dudley P. Jackson,* J. Richard Gaint Ner and Philip Zieve, Baltimore, Md.

During storage, platelets lose their ability to support clot retraction more rapidly than can be attributed to a decrease in the number of morphologically intact platelets. Platelet-rich plasma, prepared from human or canine blood collected in plastic bags containing acid citrate dextrose, was stored at $4^{\circ} \mathrm{C}$. At intervals, the degree of retraction of aliquots of the plasma was measured after addition of thrombin, and the clots were fixed in formalin. The degree of retraction and the number of morphologically intact platelets remained constant for approximately 7 days. Sections of the retracted clots showed that the platelets had undergone extensive morphologic changes of viscous metamorphosis, including swelling, pseudopodial formation, and aggregation. The fibrin strands appeared oriented to the platelets. During the second week of storage, the degree of retraction progressively decreased but the number of intact platelets fell only slightly. Clot sections showed that as the degree of retraction diminished, the number of platelets undergoing viscous metamorphosis progressively decreased, and there was a concomitant increase in the proportion of small, discrete, rounded platelets. The fibrin strands appeared oriented to the swollen and aggregated platelets but not to the small rounded platelets. Clots of plasma stored for 21 days did not retract after addition of thrombin even though more than $60 \%\left(>200,000 / \mathrm{mm}^{8}\right)$ of the platelets appeared intact. In sections of these unretracted clots, the platelets were small and round, and none appeared to have undergone viscous metamorphosis. These studies indicate that loss of the ability of stored platelets to support clot retraction is due to progressive loss of functionally active platelets and not to simultaneous, gradual loss of activity of all of the platelets.

The Regulation of Splecn Growth and Sequestering Function. Harry S. Jacob, Richard A. MacDonald and James H. Jande,* Boston, Mass.

The mechanisms regulating the growth of reticuloendothelial tissue are obscure. That growth of such erythroclastic tissue is influenced by its "work load" is suggested, because splenomegaly often accompanies hemolytic anemias owing to intrinsic defects of red cells and, in animals, splenic atrophy may follow repeated venesections. This subject was investigated by studying the growth, histology, and sequestering function of spleens, livers, and spleen autotransplants in four kinds of rats: sham-operated; splenectomized; splenectomized, with small, subcutaneously-placed spleen autotransplants; and hemisplenectomized, with similar autotransplants. The function of these various erythroclastic organs was assessed by injecting an "excess" of $\mathrm{Cr}^{51}$-labeled red cells that had been so altered in vitro that they were susceptible to sequestration by the normal spleen and liver. The growth of spleen transplants and their capacity to sequester altered red cells was three times as great in splenectomized as in 
hemisplenectomized rats. The increased size of transplants in splenectomized animals reflected hyperplasia of reticuloendothelial cells. Administration of actylphenylhydrazine induced giant transplants with heightened sequestering function of thrombocytopenia. The presence of hyperplastic spleens inhibited the sequestration of red cells by the liver, despite an excess of altered cells in the circulation, whereas in the absence of spleen tissue the sequestering capacity of the liver was accentuated. These studies demonstrate that the size of the reticuloendothelial system and its functional capacity with respect to sequestering red cells are governed by the work required of it. This may be through the extent of stimulation of reticuloendothelial cell division by sequestered particles. They suggest that splenic hyperplasia caused by an abnormality of one type of blood cell may produce increased sequestration of another. Finally, a deficiency of spleen tissue appears to induce compensatory "hyperhepatism," a phenomenon that may account for relapses after splenectomy in hemolytic anemias.

Influence of Growth on Total Urinary Hydroxyproline. Hugo E. Jasin, Chester Fink, J. Donald Smiley ANd Morris ZIFF,* Dallas, Tex.

Increased excretion of urinary hydroxyproline peptides in children as compared with adults has been previously demonstrated in this laboratory. This has suggested a relationship between the level of excretion of these peptides and growth. To investigate this phenomenon further, excretion of hydroxyproline peptides has been studied in dwarfism and acromegaly. Mean 24-hour excretion of total hydroxyproline was $33.8 \mathrm{mg}$ in 23 normal and hospitalized adults. In 34 normal and hospitalized children, 5 to 17 years of age, it was $74.3 \mathrm{mg}$. Excretion was reduced in 8 children with dwarfism, ranging between 5.6 and $28.1 \mathrm{mg}$ daily. An 8 year old pituitary dwarf was subjected to a controlled metabolic study. Before treatment with growth hormone (GH), hydroxyproline excretion averaged $14.1 \mathrm{mg}$ daily, onefifth the average of normal children of the same age. Human GH was administered in a dosage of $2.5 \mathrm{mg}$ daily for 28 days. Growth occurred, and there was a dramatic rise in excretion to a maximum of $63.5 \mathrm{mg}$ per day. Twenty-four days after cessation of therapy, urinary hydroxyproline had fallen to $18.6 \mathrm{mg}$. In an infant cretin, excretion was $5.6 \mathrm{mg}$; after 7 weeks of treatment with thyroid extract it had risen to. $47.5 \mathrm{mg}$. The mean excretion of a patient with acromegaly was $101.5 \mathrm{mg}$, three times the average for normal adults. Two months after irradiation it had fallen to $86.0 \mathrm{mg}$, and after 3 months to $58.3 \mathrm{mg}$. In view of the 1) elevated excretion of total hydroxyproline in children as compared with adults, 2) low excretion observed in nongrowing children, 3) rising values after administration of $\mathrm{GH}$ and thyroid extract, 4) increased values in a patient with acromegaly and subsequent fall after irradiation, it is concluded that there is a relationship between the excretion of hydroxyproline peptides and growth. It is suggested that this reflects the amount of metabolically active, soluble collagen in the body.

Mechanism of Renal Effects of Glucocorticoids in Cirrhosis. Hershel Jick, Joseph L. Cohen and Robert S. Morrison, Boston, Mass. (introduced by Joseph $M$. Hayman).

Depression of maximal urinary concentrating ability $\left(U_{\max }\right)$ occurs regularly in patients with cirrhosis. The $\mathrm{U}_{\max }$ was similar in six patients receiving at least 45 $\mathrm{mEq}$ sodium per day $[665 \pm 40 \mathrm{mOsm} / \mathrm{L}(1 \mathrm{SE})]$ as compared with nine patients receiving $9 \mathrm{mEq}$ of sodium per day $(614 \pm 29 \mathrm{mOsm} / \mathrm{L})$. However, while the former group exhibited a normal $\mathrm{T}_{\mathrm{e}}{ }^{\mathrm{m}} \mathrm{H}_{2} \mathrm{O}(5.5 \pm 0.78 \mathrm{ml} /$ $\mathrm{min}$ ), patients on marked sodium restriction had a low $\mathrm{T}_{\mathrm{e}}{ }^{\mathrm{m}} \mathrm{H}_{2} \mathrm{O}(2.5 \pm 0.43 \mathrm{ml} / \mathrm{min})$. In the latter group (after control values were obtained) the effects of acute ( 5 patients) and chronic ( 3 patients) administration of methyl prednisolone were studied. Administration of methyl prednisolone for 8 days was associated with a rise in $\mathrm{U}_{\max }$ of 300,120 , and $220 \mathrm{mOsm}$ per $\mathrm{L}$, and a rise in $\mathrm{T}_{\mathrm{e}}{ }^{\mathrm{m}} \mathrm{H}_{2} \mathrm{O}$ of $2.3,3.0$, and $4.7 \mathrm{ml}$ per minute, respectively. Values returned to control levels 1 week later. Drug administration was associated with a fall in $\mathrm{C}_{\mathrm{In}}$ in two of the three patients. The five patients studied acutely were in the dehydrated state, two without mannitol, and three in a steady state of mannitol diuresis, with exogenous vasopressin. Intravenous steroid administration resulted within 3 hours in a small increase in urine volume ( 0.18 and $0.24 \mathrm{ml} / \mathrm{min}$ ) in the dehydrated state alone, but a large increase $(2.7,4.3$, and $5.0 \mathrm{ml} / \mathrm{min})$ during steady mannitol diuresis. This rise in volume was accompanied by an initial rise in urine osmolality, resulting in an increased $\mathrm{T}_{e}{ }^{m} \mathrm{H}_{2} \mathrm{O}$. The $\mathrm{C}_{\mathrm{In}}$ remained unchanged or fell slightly in four of the five patients. The increased $\mathrm{T}_{\mathrm{c}}{ }^{\mathrm{m}} \mathrm{H}_{2} \mathrm{O}$ was independent of solute excretion and filtered or excreted sodium. The rise in volume and $\mathrm{T}_{c}{ }^{\mathrm{m}} \mathrm{H}_{2} \mathrm{O}$ without a rise in $\mathrm{C}_{\text {In }}$ after acute steroid administration strongly suggests that glucocorticoids inhibit proximal reabsorption of fluid, and also, either directly or by increasing the efficiency of the sodium pump in the loop of Henle, enhance maximal antidiuretic hormone effect.

\section{Studies on Iron Transport in Surviving Intestinal Seg- ments of the Rat. Jose M. JoB AND Doris A. Howell, Durham, N. C. (introduced by Jerome S. Harris).}

Contradictory conclusions concerning the intestinal transport of iron using the "in vitro" surviving intestinal segment technique of Wiseman and Wilson have been reported. Using $\mathrm{Fe}^{\mathrm{s0}}$, we have found that the concentration of radioiron on the serosal side $\left(C_{s}\right)$ of the rat duodenum and proximal jejunum becomes greater than that on the mucosal side $\left(\mathrm{C}_{\mathrm{m}}\right)$ after 3 hours of incubation in fluid of initially equal $\mathrm{C}_{\mathrm{s}}$ and $\mathrm{C}_{\mathrm{m}}$. However, the total amounts of radioiron in both serosal and mucosal compartments decrease during the incubation. The volumes change but slightly. Although the presence of a concentration gradient is suggestive of iron transport, failure to 
find an actual accumulation of $\mathrm{Fe}^{\mathrm{s \theta}}$ in the serosal compartment leaves the proof of active transport by this technique still unsettled. Difficulties in the interpretation of ferrokinetic data obtained by this technique will be discussed.

Expcrimental Coxsackie A-21 (Coe Agent) Infection in Volunteers. KarL Johnson, Anderson SpICKARD and Hugr Evans, Bethesda, Md. (introduced by Vernon Knight).

Recent evidence that Coxsackie A-21 was the cause of a large outbreak of respiratory illness in a military population suggests that it may be a more frequent cause of human illness than was previously appreciated. To supplement epidemiological studies, experiments in 50 adult volunteers were carried out. They revealed an acute influenza-like illness in susceptible volunteers given a moderate intranasal dose of the agent, but subjects with high titers of naturally acquired antibody exhibited immunity to challenge as measured by virus recovery or production of illness. By means of a quantitative measure of nasal secretion it was found that inoculated volunteers with low titers of pre-existing antibody developed a common cold syndrome with a profuse rhinorrhea, while those with no antibody developed a more severe systemic illness, often with fever but with little or no nasal discharge. Despite its designation as an enterovirus, Coxsackie A-21 was shed more profusely and for a longer period in the throat than in the feces. In corroboration of this apparent great affinity for the pharynx, inoculation of the agent into the small bowel (enteric-coated capsules or tube) produced only sporadic viral infection, no illness, and no antibody response. Thus, by all the criteria examined, Coxsackie A-21 appeared to be primarily a respiratory viral agent, a finding that is in contrast to the implication from its present classification as an enterovirus. This is but one of a number of such inconsistencies, and study of a larger number of new agents in volunteers may permit some rationalization of data on host-parasite relationships with those derived from laboratory studies now used for virus classification.

\section{Effect of Parathyroid Hormone on Bone Collagen} Metabolism In Vitro. C. Conrad Johnston, JR., Edward B. Miner and William P. Deiss, Jr.,* Indianapolis, Ind.

Both the clinician and the histologist have observed changes in bone matrix produced by parthyroid hormone $(\mathrm{PTH})$. In order to study changes in collagen metabolism of matrix at a biochemical level, calvaria from control rats and from rats injected with parathyroid extract (PTE; $75 \mathrm{U}$ every 2 hours for 3 doses) were incubated in Krebs-Ringer bicarbonate media containing $1 \mu \mathrm{c}$ L-proline- $\mathrm{C}^{14}$, and the specific activity (SA) of matrix hydroxyproline was determined. Active incorporation of the label $(74 \mathrm{cpm} / \mu$ mole $)$ is seen in an atmosphere of $\mathrm{O}_{2}$. Incorporation is enhanced $50 \%$ by adding glucose to the media, inhibited $95 \%$ by anaerobiosis and
$98 \%$ by iodoacetate $(0.001 \mathrm{M})$, and is essentially unchanged by fluoroacetate $(0.002 \mathrm{M})$. Bones from the PTE-injected rats demonstrate an early marked decrease in incorporation of labeled proline into the hydroxyproline. Two hours after the last injection the SA was $57 \%$ of control, and at 12 hours it was $17 \%$ of control. The SA of the hydroxyproline of the experimental group had returned to control level at 36 hours and had increased to $142 \%$ of control at 72 hours. The addition of glucose to the media stimulated uptake in both groups, but the differences were maintained. These data indicate that normal bones can incorporate proline- $\mathrm{C}^{\mathbf{1 4}}$ into the hydroxyproline of matrix in vitro without addition of substrate. Bones undergoing $\mathrm{PTH}$-induced reabsorption show a marked impairment of incorporation of the label. This is in contrast to the stimulated incorporation of glucose-2- $\mathrm{C}^{14}$ into matrix hexosamine noted in bones of similarly treated animals. Both observations demonstrate early metabolic changes in bone matrix after the injection of PTE.

Characteristics of the Genetic Code. Oliver W. Jones, J. Heinrich Matthaei and Marshall W. NirenBERG, Bethesda, Md. (introduced by Ivan W. Brown, Jr.).

Knowledge of inheritable metabolic disease in man must be preceded by determination of the mechanism of protein synthesis and the steps by which genetic instruction for this synthesis is transmitted. We have recently developed a system that provides a sensitive assay for "messenger" RNA. The introduction of enzymically prepared polyribonucleotides into the system has resulted in detailed information regarding coding units corresponding to 15 amino acids. Characteristics of the code such as degeneracy, the existence of nonsense "words," the minimal coding ratio, and universality of the code were investigated. Polyuridylic acid specifically stimulates incorporation of $\mathrm{L}$-phenylalanine into poly-L-phenylalanine. Incorporation of 14 other amino acids into protein was stimulated by a series of polymers containing different ratios of uridine:adenine, uridine:cytosine, uridine:guanine, and uridine:cytosine:guanine. Since coding units corresponding to four of these amino acids require three nucleotides, the minimal coding ratio must be three. Two coding units for leucine were found which demonstrated that part of the code is degenerate. Certain polynucleotides did not code for any amino acids, suggesting the presence of "nonsense" coding units. Marked similarity between RNA coding units of two phylogenetically dissimilar species indicated that part of the code is universal.

\section{The Pharmacologic Response of the Human Esophagus} to Curare and Atropine. Paul A. Kantrowitz, M. Jerome Strong, Charles I. Stegel and Donald Stephenson, Army Chemical Center and Baltimore, Md. (introduced by Albert I. Mendeloff).

Normal esophageal peristalsis progresses continuously through the upper striated muscle and lower smooth 
muscle segments; moreover, the two types of esophageal musculature cannot be distinguished by studying normal swallowing. Since esophageal involvement in neuromuscular diseases has been demonstrated, ability to distinguish between esophageal striated and smooth muscle would be useful. Intraesophageal manometric studies have therefore been performed to determine whether the two musculatures differ in their response to pharmacologic stimulation. Pharmacologic agents selected were atropine, an anticholinergic known to inhibit smooth muscle, but without striated muscle effect, and $d$-tubocurarine, a striated muscle inhibitor without smooth muscle effects in usual doses. Earlier studies in these laboratories demonstrated that peristaltic amplitude in the lower esophagus is diminished by atropine in doserelated fashion, while upper esophageal peristalsis is unaltered. In the present study, simultaneous intraluminal pressures were recorded, employing three opentipped catheters with tips $5 \mathrm{~cm}$ apart and the upper tip 1 to $2 \mathrm{~cm}$ below the upper esophageal sphincter. Six normal adult males were studied, before and after intravenous administration of 9 to $10.5 \mathrm{mg} d$-tubocurarine chloride. After curare, peristaltic amplitude in the striated muscle segment decreased by 30 to $50 \%$, while amplitude in the smooth muscle portion was unaffected. Intravenous administration of $1 \mathrm{mg}$ atropine then abolished smooth muscle peristalsis, without additional striated muscle effect. After intravenous neostigmine, peristaltic activity in both striated and smooth muscle returned to normal. These studies demonstrate that, although the striated and smooth muscle segments are indistinguishable in normal esophageal peristalsis, they do differ markedly in their pharmacologic response.

Immunological Studies with Intrinsic Factor in Man. Manuel E. Kaplan, Ralph Zalusky, Jack Remington AND Victor Herbert,* Boston, Mass.

It has been suggested that refractoriness to orally administered hog intrinsic factor concentrate (HIFC) in patients with pernicious anemia may be immunologically determined. To test this hypothesis parenteral immunization with purified HIFC was carried out in two patients with pernicious anemia, who had demonstrated their ability to absorb vitamin $B_{12}$ when it was administered with HIFC, and in albino rabbits. Control and postimmunization sera were tested for anti-intrinsic factor activity in vitro and in vivo. Despite the development, with immunization, of immediate skin sensitivity and weakly precipitating serum antibodies demonstrable by agar diffusion techniques, no refractoriness to orally administered HIFC appeared in either patient. However, the feeding of $40 \mathrm{ml}$ of autogenous immune serum with HIFC- $-\mathrm{Co}^{80} \mathrm{~B}_{12}$ prevented $\mathrm{B}_{12}$ absorption in one patient and diminished it in the other. Under the same conditions, $10 \mathrm{ml}$ of immune rabbit serum, containing high-titer precipitating antibodies of HIFC, totally suppressed $\mathrm{Co}^{60} \mathrm{~B}_{12}$ absorption. Immune, but not control, sera from both human and rabbit inhibited HIFC-enhanced uptake of $\mathrm{Co}^{60} \mathrm{~B}_{12}$ by liver and gut homogenates and prevented the migration of a HIFC- $\mathrm{Co}^{57} \mathrm{~B}_{12}$ complex on paper electrophoresis. Serum from 5 of 6 patients refractory to HIFC did not possess any of the in vitro properties described above and exhibited by the immune sera. Serum from the other refractory patient possessed all these in vitro properties except one: it failed to precipitate HIFC in agar diffusion systems. These studies demonstrate that the presence of serum antibody to HIFC does not necessarily affect the pharmacologic activity of HIFC within the gastrointestinal tract. The refractory state may be mediated by local factors within the intestinal wall, possibly including cell-fixed antibody.

Studies of an Antigen in Cell Walls of Group A Streptococci Possessing an Immunologic Relationship to Human Heart Tissue. Melvin H. Kaplan,* Cleveland, Ohio.

Experiments employing rabbit antisera to cell walls have given evidence of a protein component in certain strains of group A streptococci, which exhibits immunologic cross reaction with human heart tissue. The present report is concerned with the properties of partially purified preparations of this streptococcal antigen, the histologic distribution of the cross-reactive antigen in human organs, and the biologic variations of the streptococcal antigen among group A strains. Partially purified preparations of antigen were derived from acid extracts of whole cells or cell walls, subjected to ribonuclease digestion, neutral salt fractionation, and chromatography on DEAE- and CM-cellulose. The active material was protein in nature, and was destroyed by trypsin, pepsin, and chymotrypsin. It could be obtained separately from the group A carbohydrate, essentially free of glucosamine or rhamnose. Active fractions contained $M$ protein activity, although the antigen was present in streptococcal strains of different Lancefield types. The cross-reactive antigen as determined by fluorescent-antibody techniques is a constituent of cardiac myofibers, skeletal muscle, and smooth muscle of vessel walls, and was demonstrable in tissues of both rheumatic and nonrheumatic individuals. The antigen was also demonstrated in rabbit heart tissue. The converse cross reaction could also be demonstrated. Antisera to human heart or rabbit heart exhibited precipitating antibodies reactive with active streptococcal fractions. The hypothesis is proposed that in rheumatic fever an autoimmune reaction to a constituent resident in human heart may be induced by exposure to a cross-reactive antigen of group A streptococci. This concept is supported by our previous observation of bound $\gamma$-globulin in myofibers and vessel walls of rheumatic hearts.

Bipolar Electrodes for Measurcment of Kidney Pulse by the Impedance Method. YALE J. KATZ, Los Angeles, Calif. (introduced by Irving Gordon).

Phasic volume change in the kidney during the cardiac cycle allows the registration of the organ pulse by an electric impedance method. The contour of the pulse wave is affected by changes in the caliber of the renal 
artery. For recording the kidney pulse, two small bipolar electrodes have been recently developed. One consists of a plastic plate containing two needle points $1 \mathrm{~cm}$ apart. At surgery the electrode plate is fixed to the kidney capsule with the electrode points in kidney cortex. The recording apparatus consists of an $\mathrm{AC}$ generator, 40,000 cycles per second, and a capacitance bridge; the signal resulting from the unbalancing of the bridge by the change in tissue impedance is fed to a DC recorder. The tracing of the kidney pulse wave resembles in form the pressure curve of a peripheral artery. The kidney pulse of a patient with significant stenosis of the renal artery has a delayed onset, a prolonged upstroke time, and some decrease in amplitude. In one patient a successful endarterectomy was followed by conversion of the kidney pulse to one of a normal contour. The second electrode consists of a bipolar unit built into the trocar of the Vim-Silverman renal biopsy needle. It has been used successfully for kidney pulse wave determination by the percutaneous route in Goldblatt hypertensive dogs. Since stenosis of the renal artery is seen at times at aortography in the absence of hypertension, one should not assume when hypertension is present that the stenosis of the artery is the cause of the hypertension. One can only properly assume this if the stenosis is a significant one in a hemodynamic sense. Kidney pulse measurement will assist in determining the functional significance of a stenosis of the renal artery.

Effect of Thyroid Hormone on Collagen Metabolism. Harry R. Keiser and Albert Sjoerdsma,* Bethesda, Md.

Body collagen, taken as a whole, has been considered to be metabolically inert in adult animals. That this concept may require revision is suggested by the present findings of the production by thyroid hormone of increased urinary excretion of peptide-bound hydroxyproline; the latter is a convenient index of collagen degradation in man. Six normal adults and a myxedematous patient were studied. During maintenance on a low hydroxyproline diet, 3,5,3'-triiodothyronine $\left(T_{3}\right)$ was given orally in dosage sufficient to produce hyperthyroidism in the normal subjects and euthyroidism in the patient. Control values for total urinary hydroxyproline were 16.4 to 40.5 mg per day in the normal subjects. A 56 to $109 \%$ increase occurred during administration of $T_{3}$ for 10 to 21 days with a return to normal within 10 days of discontinuing treatment. In all instances free hydroxyproline was less than $5 \%$ of the total. Other changes during the period of hypermetabolism were as follows: caloric intake, 0 to $+31 \%$; protein intake, 0 to $+41 \%$; total urine nitrogen, +8 to $+65 \%$; free urine $\alpha$-amino acid nitrogen, +7 to $+30 \%$; and free urine tyrosine, +30 to $+61 \%$. In the hypothyroid patient, control hydroxyproline values averaged $3.1 \mathrm{mg}$ per day, a very low level. An increase to $15.4 \mathrm{mg}$ per day $(+397 \%)$ occurred after 21 days on $\mathrm{T}_{3}$ in the presence of a constant protein intake, total urinary nitrogen increasing only $8.8 \%$. These data indicate that collagen, as well as general protein metabolism, was altered to a marked degree by $T_{3}$ administration. The rapid and marked increase in urinary hydroxyproline could have resulted from increased synthesis of a rapidly metabolized form of collagen (i.e. soluble collagen), or increased degradation of collagen, or a combination of both. The precise mechanism is under investigation.

Studies on the Role of the Brain in the Steroidal Inhibition of ACTH Release. JoHn W. Kendall, Kunio Matsuda, Caroline Duyck and Monte A. Greer,* Portland, Ore.

Previous studies have provided conflicting evidence regarding the site of the receptor area at which adrenocortical steroids inhibit ACTH release. To evaluate the role of the brain in this mechanism, the effect of corticosteroid administration on pituitary-adrenal function was determined in rats after removal of graded amounts of brain. A "hypothalamic island" group was prepared by removing the cerebral hemispheres, thalamus, and hypothalamic connections to the posterior brain. "Median eminence island" and "isolated pituitary" groups were prepared by additional subtotal or total extirpation of the hypothalamus. Half of each group was given $100 \mu \mathrm{g}$ dexamethasone subcutaneously 20 hours postoperatively; after another 4 hours adrenal corticosterone secretion rates were measured by fluorescences assay of a 3-minute plasma specimen obtained by left adrenal vein cannulation. Secretion rates were high in untreated intact and "hypothalamic island" groups owing to the stress associated with obtaining the specimen. Approximately half of the untreated "median eminence island" and "isolated pituitary" animals had similarly high secretion rates indicating that they too responded to stress. In contrast, adrenal secretion was markedly supressed in all animals receiving dexamethasone. These findings indicate that administration of adrenocortical steroids prevents ACTH release in rats stressed by adrenal vein cannulation even though the entire brain anterior to the superior colliculus and superior to the pituitary has been removed. This portion of the central nervous system therefore does not seem an essential part of the steroidal feedback mechanism.

Intracellular Acid-Base Relations of Dog Brain with Reference to the Extracellular Volume of the Brain. R. F. Kibler, R. P. O'NeIll and E. D. Robin,* Pittsburgh, $\mathrm{Pa}$.

Brain intracellular $\mathrm{pH}\left(\mathrm{pH}_{1}\right)$ was determined in vivo in dogs by the DMO (5,5-dimethyl 2,4-oxazolidinedione) technique under control circumstances and after the administration of $\mathrm{NaHCO}_{3}, \mathrm{HCl}$, and $\mathrm{CO}_{2}$. The DMO technique requires estimation of the extracellular fluid space (ECF) of the brain. Since there is no general agreement concerning the magnitude of this space, both chloride and sulfate spaces were measured as estimates of brain ECF. These spaces averaged $41 \pm 4 \%$ and $6 \pm$ $1 \%$ of total brain water, respectively. Since calculations 
based on chloride space frequently resulted in negative values for intracellular DMO, chloride space could not be used to determine brain $\mathrm{pH}_{1}$. In 21 dogs under control conditions the mean sulfate space-derived $\mathrm{pH}_{1}$ was $7.01 \pm 0.12$ at a plasma $\mathrm{pH}$ of $7.36 \pm 0.08$. One hour after $\mathrm{NaHCO}_{3}$ infusion in 7 dogs, mean plasma $\mathrm{pH}$ rose $0.33 \pm 0.08 \mathrm{U}$ while $\mathrm{pH}_{1}$ rose $0.12 \pm 0.07 \mathrm{U}$; one hour after $\mathrm{HCl}$ infusion in 4 dogs, plasma $\mathrm{pH}$ fell $0.28 \pm 0.13 \mathrm{U}$ while $\mathrm{pH}_{1}$ fell $0.04 \pm 0.03 \mathrm{U}$. Inhalation of $10 \% \mathrm{CO}_{2}$ for 1 hour in 2 dogs caused a fall in plasma $\mathrm{pH}$ of 0.40 and $0.45 \mathrm{U}$ and a fall in $\mathrm{pH}_{1}$ of 0.35 and $0.39 \mathrm{U}$, respectively. These results suggest that sulfate space is a more reasonable estimate of brain ECF than is chloride space. The value of $6 \%$ is in close agreement with recent estimates of brain ECF based on electron microscopic observations. Accordingly, it appears that much of the brain chloride is intracellular, possibly within glial cells, as previously proposed by others. The mean sulfate space-derived intracellular $\mathrm{pH}$ of 7.01 is similar to that obtained for muscle and for mean whole body intracellular $\mathrm{pH}$. Brain cells also behave like muscle cells in their slow equilibration with $\mathrm{HCO}_{8}^{-}$and $\mathrm{H}^{+}$ions and rapid equilibration with $\mathrm{CO}_{2}$.

Intrinsic Renal Regulation of Sodium Reabsorption. FreDrIK KIIL, Dallas, Tex. (introduced by Donald W. Seldin).

Micropuncture studies have demonstrated that the thick ascending limb functions as an obligatory diluting segment. To test the hypothesis that this segment reduces the concentration of tubular sodium to a hypotonic level irrespective of sodium delivery, the quantity of sodium reaching this segment in anesthetized dogs was increased either by raising glomerular filtration rate (GFR) or reducing proximal tubular reabsorption. Free water clearance $\left(\mathrm{C}_{\mathrm{H}_{2} \mathrm{O}}\right)$ was used as an estimate of sodium reabsorption in this water-impermeable area. Starting with water diuresis with minimal urine osmolal concentration (50 to $60 \mathrm{mOsm} / \mathrm{kg}$ ), $\mathrm{C}_{\mathrm{H}_{2} \mathrm{O}}$ more than doubled when administration of hypotonic mannitol increased urine flow to two-thirds of GFR. When GFR was increased by administration of hypotonic saline, $\mathrm{C}_{\mathrm{H}_{2} \mathrm{O}}$ increased to $12 \mathrm{ml}$ per minute, nearly one-third of the GFR obtained during control water diuresis, or $18 \%$ of actually filtered volume. Unchanged distal sodium reabsorption assumed, sodium concentration of fluid issuing from the thick ascending limb did not exceed $80 \mathrm{mEq}$ per $\mathrm{L}$ at maximal flow rates. Sodium reabsorbed in the waterimpermeable segment was calculated as half the product of $\mathrm{C}_{\mathrm{H}_{2} \mathrm{O}}$ and osmolal concentration of serum. During hypotonic mannitol diuresis sodium reabsorption decreased in proximal tubules and increased in the waterimpermeable segment. In this segment a larger increase in sodium reabsorption occurred during hypotonic $\mathrm{NaCl}$ diuresis, in accordance with hypothesis. These observations suggest that the thick ascending limb always functions to reduce the sodium concentration to a low value, irrespective of the rate of sodium delivery, and would thereby explain why changes in filtration rate or serum sodium concentration result in corresponding directional changes in tubular sodium reabsorption.

Electroencephalographic Evaluation of Uremia. ЈоHN E. Kiley and Orlando Hines, Albany, N. Y. (introduced by Richard T. Beebe).

Dialytic therapy has been hampered by inadequate objective criteria of the severity of uremia. Patients in uremia gradually lose their ability to think. Since this impairment has been a helpful indication for dialysis, electroencephalographic changes were investigated as an index of the degree of uremia. Electroencephalograms were performed on 16 uremic patients and serial electroencephalograms were followed in 13 . Patients were evaluated clinically with particular regard to mental state and grouped as follows: A, alert and rational; B, lethargic or toxic; C, irrational (definite impairment of thinking); $\mathrm{D}$, semicomatose (responding only to pain); $\mathrm{E}$, comatose (completely unresponsive). Changes in blood urea nitrogen and creatinine were followed as well as the accumulation of generally unidentified anions, estimated as the difference of serum sodium plus potassium minus bicarbonate plus chloride concentrations. The diffuse slowing of the electroencephalogram observed in uremia was quantitated by enumeration in occipital leads of both the per cent distribution of various frequencies and the per cent of frequencies that were abnormal. The electroencephalogram slowed and the percentage of abnormal frequencies increased as uremia became worse, and these changes reversed when uremia improved. Either hemodialysis or peritoneal dialysis decreased the percentage of abnormal frequencies. The electroencephalogram was a more sensitive indication of the effect of uremia than was our evaluation of mental status. Of seven patients with $90 \%$ or more of frequencies abnormal, all died except for two promptly treated by dialysis. Correlation between biochemical measurements and slowing of the electroencephalogram was poor. It is concluded that quantitative estimation of slowing of the electroencephalogram by uremia provides a needed index of the net effect of complex metabolic derangement on central nervous system function.

Studies to Determine whether Glucose Must Be Metabo-
lized to Induce Insulin Release. Charles KIlo,
Charles L. Long, JR., Rita M. BaIley, Mary B. Koch and Lillian Recant,* St. Louis, Mo.

Previous investigations using isolated pancreas, crosscirculation experiments in dogs, intravenous infusions of glucose, and measurement of pancreaticoduodenal vein blood samples in dogs have shown that glucose is capable of inducing insulin release from the pancreas. The purpose of this investigation was to determine 1) whether penetration of glucose into the $\beta$-cell was necessary for stimulation of insulin release; 2 ) the metabolic step in glucose utilization inducing insulin release. Substances tested were infused into pancreaticoduodenal and splenic arteries in intact anesthesized dogs. Portal venous speci- 
mens were obtained before and after infusions. Insulinlike activity (ILA) was measured in portal samples by a modification of the rat epididymal fat-pad assay. Experiments were performed on 62 dogs. Perfusion of saline or pyruvate resulted in no increase in ILA. Glucose, fructose, or tolbutamide produced significant increases in ILA (600 to $400 \mu \mathrm{U} / \mathrm{ml}$ ); 2-deoxyglucose, a sugar metabolized only to 2-deoxyglucose-6-phosphate, resulted in no ILA response. Since 2-deoxyglucose6-phosphate inhibits the subsequent metabolism of glucose-6-phosphate, simultaneous infusions of glucose and 2-deoxyglucose were studied. Complete inhibition of insulin release occurred when the glucose to 2-deoxyglucose blood levels were 1.5 or less. Delayed but significant ILA increases were observed when ratios were increased. 2-Deoxyglucose infusions resulted in increased glucose $\mathrm{A}-\mathrm{V}$ differences for 15 to 30 minutes, followed by prolonged inhibition of glucose uptake. These studies show that glucose, fructose, and tolbutamide, but not 2-deoxyglucose or pyruvate, are capable of stimulating insulin release. They further suggest that glucose must penetrate the $\beta$-cell and be metabolized beyond the glucose6-phosphate step in order that insulin be released. Studies are under way 1) to test additional utilizable and nonutilizable sugars; 2) to test metabolic products of glucose; and 3 ) to evaluate the effect of 2-deoxyglucose on release of ILA by tolbutamide.

Site of Action of Androgen on Adrenal Steroidogenesis. Julian I. Kitay, Charlottesville, Va. (introduced by Alfred Jay Bollet).

The influence of androgen on steroidogenesis was studied in adult intact and castrated male rats. Adrenal tissue for intact animals produced $0.031 \pm 0.002 \mu$ mole (per $100 \mathrm{mg}$ adrenal weight) of corticosterone when incubated in vitro without ACTH. A significant decrement to $0.023 \pm 0.001 \mu$ mole per $100 \mathrm{mg}$ was observed in adrenal slices from castrated rats. Testosterone administration to castrated rats in vivo enhanced in vitro steroidogenesis to a mean value of $0.040 \pm 0.002 \mu$ mole per $100 \mathrm{mg}$. Studies were then undertaken to explore the factors involved in this stimulatory effect of androgen. Adrenal glycogen concentration (as glucose) was found to be $0.758 \pm 0.069 \mu$ mole per $100 \mathrm{mg}$ in intact rats. Castration resulted in a $58 \%$ reduction to $0.317 \pm$ $0.052 \mu$ mole per $100 \mathrm{mg}$. Adrenal cholesterol levels were $8.94 \pm 0.65 \mu$ moles per $100 \mathrm{mg}$ in the intact animals and $13.73 \pm 1.14 \mu$ moles per $100 \mathrm{mg}$ after castration, an increment of $53 \%$. The concentrations of three enzymes in adrenal tissue-phosphorylase, phosphodiesterase, and 5 '-nucleotidase-were found to be reduced significantly by castration (decrements of 16,32 , and $24 \%$ per $\mathrm{mg}$ of adrenal protein, respectively) and restored to normal levels with testosterone replacement. On the other hand, adrenal glucose-6-phosphate dehydrogenase activity was actually somewhat enhanced by castration, and ATPase was unchanged. Finally, the decrement in steroidogenesis observed in adrenal tissue from castrated rats was not obtained when adrenal homogenates were prepared and incubated in the presence of excess TPNH. ACTH administration has been shown previously to increase the activity of adrenal phosphorylase, phosphodiesterase, and 5 -nucleotidase. However, in the present studies, the addition of a supramaximal dose ( $0.5 \mathrm{U})$ of ACTH in vitro did not alter the decrease in adrenal steroidogenesis produced by castration. These data suggest that testosterone affects steroidogenesis by stimulation of the pathway leading to increased generation of TPNH. This stimulation is analogous to, but not identical with, that produced by ACTH.

Urea Transport in the Central Nervous System. CharLeS

R. Kleeman,* Hugh Davson and Emelio Levin, London, England and Los Angeles, Calif.

Studies on urea transport in the central nervous system of rabbits were done to investigate the mechanism of its ability to lower cerebrospinal fluid (CSF) pressure. Information was obtained on the site of the "barrier" to urea penetration and on the presence of a dynamic extracellular space of the brain. Sustained intravenous and intracisternal infusions of $\mathrm{C}^{12}$ - and $\mathrm{C}^{\mathbf{1 4}}$-urea were administered for 4 hours to normal rabbits; the rate of $\mathrm{C}^{14}$-urea penetration in the brain, cord and CSF was followed in nephrectomized rabbits up to 24 hours. The preinfusion content of urea in the water of cord and white matter equaled that of plasma water. Urea content of gray matter was $18 \%$ higher than in plasma, while urea in CSF was only 0.7 to 0.8 the plasma concentration. Despite the latter, the CSF had the same urea concentration as the choroidal secretion (ventricular fluid). Blood urea maintained at 200 to $300 \mathrm{mg}$ per $100 \mathrm{ml}$ caused a 7 to $12 \%$ decrease in water content of CNS tissues without affecting rate of CSF formation. Dehydration of the brain was the major cause of the fall in CSF pressure. Urea penetrated from blood to CNS tissues aproximately one-tenth as rapidly as into muscle. The osmotic pressure differential explained tissue dehydration. It required 15 to 24 hours for plasma to tissue urea ratio to reach 1 . Urea infused into subarachnoid space penetrated CNS tissues four to five times more rapidly than when infused intravenously. Water content of brain increased during subarachnoid perfusion. Because of these results with urea, sucrose "space" in brain was compared after i.v. and subarachnoid infusions of sucrose; i.v. sucrose space was less than $2 \%$ of brain, while subarachnoid infusion space was 10 to $14 \%$. Urea lowers CSF pressure by decreasing brain volume. The major barrier to penetration is at capillary, not glial or neuronal cell membrane. A sucrose "space" exists when capillary is "bypassed" by subarachnoid perfusion.

Physiological Changes of Erythrocyte Carbonic Anhydrase Activity. Samuel Korman and Marianne Kara, New York, N. Y. (introduced by Robert G. Bloch).

The interaction of erythrocyte carbonic anhydrase with $\mathrm{Ca}^{++}, \mathrm{K}^{+}$, acetazolamide, digoxin, and cortico- 
steroids was studied in vitro and in vivo. The addition of $\mathrm{Ca}^{++}$or $\mathrm{K}^{+}$to normal heparinized blood resulted in a $21 \%$ increase in enzyme activity. $\mathrm{Mg}^{++}$increased the enzyme activity only in the blood of patients with hypoparathyroidism. The bloods obtained from twothirds of normal individuals had an average enzyme activity increase of $20 \%$ when incubated with digoxin. Acetazolamide $(0.05 \mathrm{mg} / \mathrm{ml})$ inhibited the activity $30 \%$. The intravenous infusion of $\mathrm{Ca}^{++}$over a 4-hour period to patients with osteoporosis resulted in a $20 \%$ increase in carbonic anhydrase activity. Hypoparathyroid patients showed an earlier and greater rise, whereas patients with hypercalcemia had only a slight (or no) increase in their enzyme activity. The blood of patients receiving corticosteroids or with Cushing's syndrome did not show an increase in their erythrocyte carbonic anhydrase activity with either $\mathrm{Ca}^{++}$or $\mathrm{K}^{+}$. Neither did the intravenous infusion of $\mathrm{Ca}^{++}$to those individuals result in an increase in activity. The incubation of hemolyzed red cells with $\mathrm{K}^{+}$and digoxin resulted in no change in enzyme activity, whereas $\mathrm{Ca}^{++}$ increased the activity approximately $13 \%$, and acetazolamide caused an even greater inhibition. Thus the intact erythrocyte is required to obtain the enhanced effect with $\mathrm{K}^{+}$or digoxin. The inhibition due to acetazolamide could be prevented partially or completely by the addition of $\mathrm{Ca}^{++}, \mathrm{K}^{+}$or digoxin to whole blood but not when added to hemolyzed erythrocytes. Advantage was taken of the antagonism between digoxin and acetazolamide in diagnosing digitalis toxicity. The inhibition of erythrocyte carbonic anhydrase was markedly decreased or absent at acetazolamide concentrations of 0.05 or $0.10 \mathrm{mg}$ per $\mathrm{ml}$ when patients were digitalis-toxic. A digitalized but not digitalis-toxic individual exhibited enzyme inhibition at the higher acetazolamide concentration; however, the inhibition might be absent or decreased at the lower concentration. These studies correlate the interaction of digitalis, acetazolamide, and corticosteroid therapy and abnormal $\mathrm{Ca}^{++}$and $\mathrm{K}^{+}$metabolism with erythrocyte carbonic anhydrase activity.

Abnormal Rise of Plasma Amino Acids and the Appearance of a Polypeptide in the Plasma in Adult Celiac Disease after Oral Administration of Gliadin. $\mathrm{O}$. Dhodanand Kowlessar, Elliot Weser and LoRRAINE J. HAEFfner, New York, N. Y. (introduced by Marvin $H$. Sleisenger).

The concentration of amino acids in the ultrafiltrates of plasma before and at 2 and 3 hours after administration of gliadin $(350 \mathrm{mg} / \mathrm{kg})$ was determined in six normal subjects and ten patients with adult celiac disease (nontropical sprue). The amino acids were separated either by bidimensional chromatography-butanol: acetic acid:water $(120: 30: 50)$, followed by butanol: pyridine: water $(70: 70: 70)$ - or by high voltage electrophoresis (HVE) at $\mathrm{pH} 2.2(4 \% \mathrm{vol} / \mathrm{vol}$ formic acid; $0.3 \%$ pyridine) and $2,000 \mathrm{v}$ for 70 minutes followed by chromatography in butanol:acetic acid:water $(120: 30: 50)$. Chromatograms were stained with nin- hydrin and the amino acids were eluted and measured colorimetrically. The results of these studies demonstrated: 1) that patients with adult celiac disease have low fasting plasma amino acid levels; 2) concentration of all amino acids in the plasma of both patients and normal subjects increased after feeding gliadin, with the peak level at 2 hours; 3 ) the percentage of increase of levels at 2 and 3 hours after gliadin was significantly higher in the patients; and 4) unlike the normal subjects, the concentration of amino acids at 3 hours in the patients was still greater than the fasting sample. After gliadin loading, the plasma ultrafiltrates were also hydrolyzed with hog intestinal peptidase, and the concentration of amino acids was redetermined. The levels of glutamine, glutamic acid, leucine, isoleucine, proline, valine, lysine, and arginine increased by more than $2 \mathrm{mg}$ per $100 \mathrm{ml}$ in the patients, while only leucine and arginine rose in the six normal subjects. Further, a ninhydrin-staining spot appeared after HVE and chromatography, which yielded glycine, serine, threonine, lysine, valine, phenylalanine, leucine or isoleucine, or both, and glutamic acid after acid hydrolysis. These findings strongly suggest that patients with adult celiac disease absorb peptides when gliadin is fed. This phenomenon indicates a deficiency or absence in these patients of intestinal peptidases which are required for the breakdown of gliadin polypeptides into amino acids.

Isoproterenol and Myocardial Function in Man. NoRman Krasnow, William B. Hood, Jr., Ellis R. Rolett, Peter M. Yurchak and Richard Gorlin,* Boston, Mass.

The effect of catecholamines on myocardial energetics has been infrequently studied in man. Ten subjects with heart disease, not in clinical failure, were studied during coronary sinus catheterization, with measurement of coronary flow $(\mathrm{CF})$, myocardial oxygen consumption $\left(Q_{\mathrm{O}_{2}}\right)$, and left ventricular ejection; and pressure dynamics at rest, during exercise, and during isoproterenol infusion ( 2 to $3 \mu \mathrm{g} / \mathrm{min}$, i.v.). Comparable mean rises were achieved during exercise or isoproterenol infusion in cardiac and stroke index, heart rate, and systolic mean pressure. Compared with rest, both states induced a fall in systolic ejection period and rise in mean systolic ejection rate (SER), somewhat greater with isoproterenol than with exercise. Systolic time per minute was unchanged with isoproterenol but increased on exercise. Pressure-time per beat (PTB) increased on exercise, but decreased during isoproterenol. Decreases in PTB were associated with rises in SER. Isoproterenol caused coronary vasodilatation: increased $\mathrm{CF}$ with decreased coronary $\mathrm{A}-\mathrm{V} \quad \mathrm{O}_{2}$ difference, $\mathrm{A}-\mathrm{V} / \mathrm{A} \mathrm{O}_{2}$, and rise in coronary venous oxygen $(+1.2$ vol \%). Such changes did not occur with exercise. Contractility was increased so that normal or increased stroke volume was delivered in a greatly shortened ejection time. Increased mean ejection velocity implies increased rate of fiber shortening. The chronotropic action of the drug, contrary to exercise, did not alter 
total systolic or diastolic time per minute. Myocardial $\mathrm{Q}_{\mathrm{O}_{2}}$ increased proportionately to pressure-time per minute (PTM) on drug and exercise. The unchanged $\mathrm{PTM} / \mathrm{Q}_{\mathrm{O}_{2}}$ at this dosage of isoproterenol can be best explained by reduction in heart size (Rushmer) thereby reducing total wall tension $\left(T=\pi r^{2} P\right)$ and counterbalancing any "energy-wasting" effects of catechols. Isoproterenol does not simulate exercise in man. This dose of drug has primary as well as secondary vasodilator actions on coronary vessels, and enhances contractility and ejection velocity without increased myocardial energy cost. The reciprocal rise in SER and fall in PTB suggest a crude analogy to the forcevelocity relation of isolated muscle.

Simultaneous Determination of Cardiac Output and Renal Hemodynamics in Decompensated Laennec's Cirrhosis. Ruben G. Lancestremere, Paul L. Davidson, Laurence E. Earley, Frederick J. O'Brien AND Solomon PAPper,* Boston, Mass. and Richmond, Va.

Cardiac output (CO), measured by $\mathrm{T}-1824$ dye or by radioiodinated albumin dilution methods, and inulin and para-aminohippurate clearances $\left(\mathrm{C}_{\mathrm{In}}, \mathrm{C}_{\mathbf{P \Delta B}}\right)$, were determined simultaneously in 20 patients with decompensated Laennec's cirrhosis. $C_{I n}$ ranged from 2 to $225 \mathrm{ml}$ per minute; $\mathrm{C}_{\mathrm{PAB}}$ ranged from 8 to $875 \mathrm{ml}$ per minute. The renal fraction of the $\mathrm{CO}$ was less than $14 \%$ in all cases. Seven cirrhotics in renal failure $\left(C_{\text {In }}\right.$ less than $60 \mathrm{ml} / \mathrm{min}$ ) had $\mathrm{CO}$ greater than 7.0 $\mathrm{L}$ per minute. One patient studied on two occasions, had a $\mathrm{C}_{\mathrm{In}}$ of $114 \mathrm{ml}$ per minute and a $\mathrm{CO}$ of $6.9 \mathrm{~L}$ per minute in the first admission; 7 months later, in his terminal hospitalization, he had a $C_{\text {In }}$ of $9 \mathrm{ml}$ per minute and a $\mathrm{CO}$ of $10.0 \mathrm{~L}$ per minute. Thus, $\mathrm{CO}$ was generally elevated in patients who exhibited a broad range of renal hemodynamics. Further, the findings indicate that the diminished glomerular filtration rate and renal plasma flow found in some patients with cirrhosis occur in the presence of an increased $\mathrm{CO}$.

Thyrotropic Hormone Action on Carbohydrate Metabolism in Thyroid Slices. Bernard R. LANDau,* WILFried Merlevede and Galen Weaver, Cleveland, Ohio.

Beef thyroid slices were incubated with a thyrotropic hormone (TSH) preparation in the presence of specifically labeled sugars. As reported by others, TSH increased the oxidation of carbons 1 and 6 of glucose to $\mathrm{CO}_{2}$; this effect was most dramatic in the first half hour of incubation. From the randomization of carbon 2 of glucose-2- $\mathrm{C}^{\mathbf{1 4}}$, as reflected in the distribution of radioactivity in glycogen, it is estimated that, of the total quantity of glucose metabolized by the thyroid slices, approximately $4 \%$ was metabolized via the pentose cycle in the absence, and $12 \%$ in the presence, of TSH. This estimation depends upon isotopic equilibration between glucose-6-phosphate and fructose-6-phosphate. Support for this assumption was obtained from the C-1 to C-6 ratio of $\mathrm{CO}_{2}$ yields on incubation of fructose-1- $\mathrm{C}^{14}$ when compared with that on incubation of glucose-1- $\mathrm{C}^{14}$ and $6-\mathrm{C}^{14}$. The increase in pentose cycle activity on TSH addition does not appear attributable to increased availability of intracellular glucose, for while increasing the concentration of glucose in the medium did result in increases in the quantity of $\mathrm{CO}_{2}$ from carbons 1 and 6 , the $\mathrm{C}-1$ to $\mathrm{C}-6$ ratio was less than that produced by TSH. TSH did cause a decrease in the incorporation of glucose into glycogen. While glucagon and insulin were without effect, epinephrine also stimulated the oxidation of glucose to $\mathrm{CO}_{2}$. The possibility remains that some or all of the effects of TSH on the thyroid may be consequent to an initial action on glycogen.

\section{Comparison of Two Applications of the Stewart-Hamilton} $(S-H)$ Principle for Measurement of Central Blood Volume. Ramon L. Lange and Hans H. Hecht,* Milwaukee, Wis. and Salt Lake City, Utah.

In calculating subsegments of the "central blood volume" (CBV), two theoretically equivalent methods for measurement of mean circulation time (MCT) gave consistently divergent results. This led to systematic comparison of CBV obtained by these two methods in 16 humans with and without heart disease. The traditional $\mathrm{S}-\mathrm{H}$ method involves slug injection of indicator into the pulmonary artery (PA) and obtaining a single dilution curve from the femoral artery (FA) for measurement of cardiac output ( $Q$ ) and MCT. Then $C B V=Q \times$ MCT. In the second method, densitometer and (as a further check) thermistor curves were obtained from the PA simultaneously with densitometer curves from FA, after a rapid venous injection of cold dye. The $\mathrm{S}-\mathrm{H}$ principle is now expressed as $\mathrm{CBV}=\mathrm{Q} \times \triangle \mathrm{MCT}$, where $\triangle \mathrm{MCT}$ is the difference between MCT of the $\mathrm{PA}$ and FA curves. Appropriate corrections were made for the delay of densitometer curves due to sampling dead space. The traditional method consistently yielded longer MCT and, therefore, CBV that averaged 380 $\mathrm{ml}$ or $31 \%$ larger than by the double-curve method $(p<0.001)$. It appears that PA slug injection (the original S-H method) does not provide for efficient pulmonary mixing and that $\mathrm{CBV}$ is overestimated. PA thermistor curves recorded with PA injection (method I) support this contention.

Angiotensin II and Renal Sodium Transport: Natriuresis and Diuresis in Patients with Cirrhosis and Ascites. John H. Laragh,* Paul J. Cannon, Richard P. Ames, Alfred M. Sicinski, Carl J. Bentzel and Jay I. Meltzer, New York, N. Y.

To study further a possible renal-adrenal interaction (angiotensin-aldosterone) for regulating sodium balance, the influences of angiotensin, compared with equipressor amounts of norepinephrine, were examined in normal subjects and in a representative type of secondary hyperaldosteronism (i.e., cirrhosis with ascites). Effects on 
aldosterone secretion, arterial pressure, electrolyte balance, and renal clearances were observed during conditions of controlled metabolism. During continuous infusions for up to 10 days, angiotensin stimulated aldosterone secretion in seven normal subjects (24 studies), but did not augment oversecretion of ten cirrhotics (24 studies). In cirrhosis there was markedly reduced pressor responsiveness to angiotensin only, indicating that increased endogenous angiotensin could account for this hyperaldosteronism without causing hypertension. In normals, both angiotensin and norepinephrine initially produced salt and water retention, then re-establishment of balance, In cirrhosis, both agents caused natriuresis and diuresis. Angiotensin caused more sodium retention in normals and more natriuresis in cirrhosis; norepinephrine promoted less water retention in normals and more free water excretion in cirrhosis. The natriuresis produced by angiotensin in cirrhosis was marked and sustained. Urinary sodium excretion rose from values of less than 1 to over $500 \mathrm{mEq}$ per day, leading to clearing of edema fluid. Effects were much greater in magnitude than could be achieved with potent diuretic agents. In ten clearances in cirrhosis, angiotensin increased mean sodium excretion by $1,367 \mu \mathrm{Eq}$ per minute. $\mathrm{C}_{\mathrm{Na}} / \mathrm{C}_{\text {In }}$ increased from 0.2 to $10.9 \%$. Norepinephrine produced lesser saliuresis of $63 \mu \mathrm{Eq}$ per minute, with increased GFR, and no change in $\mathrm{C}_{\mathrm{Na}} / \mathrm{C}_{\mathrm{In} \text {. Angiotensin }}$ thus markedly inhibited tubular reabsorption of sodium chloride. Effects on $\mathrm{C}_{\mathrm{osm}}$ and $\mathrm{C}_{\mathrm{H}_{2} \mathrm{O}}$ indicated that both proximal and distal tubular transport processes were depressed. The results suggest an intrarenal role for angiotensin in regulating sodium reabsorption. The magnitude of the changes indicates that such an action of angiotensin could be more important than its previously described effect on aldosterone release.

In Vitro Endocrine Stimulation of Active Transport in the Small Intestine of Hamster. LEONARD LASTER ANd David Danoff, Bethesda, Md. (introduced by Donald S. Fredrickson).

Animal studies in vivo by Althausen indicated that "thyroid hormone has a direct stimulating effect on the preferential intestinal absorption" of glucose, but not of alanine; that adrenocortical hormones do not exert a direct effect on intestinal absorption; and that hyperthyroidism in man increases glucose absorption. As part of a systematic investigation of in vitro endocrine effects on small-intestine function and metabolism, we have demonstrated that $3,5,3^{\prime}-\mathrm{L}$-triiodothyronine $\left(\mathrm{T}_{8}\right)$ and L-thyroxine $\left(T_{4}\right)$ increase the rate of transport against a concentration gradient of D-glucose (metabolized) and 3-deoxy-D-glucose (minimally metabolized) by surviving everted segments of small intestine of hamster. Glucose transport was not altered by 3,5-Ldiiodotyrosine $\left(10^{5-}\right.$ and $\left.10^{-4} \mathrm{M}\right)$. $\mathrm{T}_{4}$ doubled the absorption rate of L-alanine. Cortisol $\left(10^{-6} \mathrm{M}\right)$ appeared to triple the D-glucose absorption rate. An individual segment was studied during consecutive periods with hormone absent, present, and again absent from solution bathing mucosal surface, thus providing experimental and control data from a single piece of tissue. Glucose transport was maximally stimulated, twofold by $T_{8}\left(10^{-5} \mathrm{M}\right)$ and three- to fourfold by $T_{4}$ $\left(2.5 \times 10^{\circ} \mathrm{M}\right)$, when the initial concentration of glucose in the solutions bathing mucosa and serosa was $2 \times$ $10^{-8} \mathrm{M}$. At lower $\left(0.5 \times 10^{-3} \mathrm{M}\right)$ and higher $(5 \times$ $\left.10^{-3} \mathrm{M}\right)$ initial glucose concentrations, stimulation was minimal. After removal of an intestinal segment from a $\mathrm{T}_{8}$-containing medium to a $\mathrm{T}_{3}$-free medium, transport rates returned to basal levels, but $T_{4}$-stimulated segments continued to transport glucose at the accelerated rate after exposure to $T_{4}$ was terminated. $T_{4}$ in solution bathing serosa produced less stimulation of transport than did $T_{4}$ in solution bathing mucosa. The observed hormonal effects cannot be attributed to alterations in water transport. Intestinal segments from animals 2 weeks after thyroidectomy were not strikingly more sensitive to $T_{3}$ than were segments from normal animals, although the basal rate of glucose transport was lower in the operated group.

Regulatory Mechanisms of Leukocyte Metabolism. JoH N Laszlo, Durham, N. C. (introduced by R. Wayne Rundles).

There are fundamental differences in the regulation of energy-yielding metabolism of lymphocytic and granulocytic normal and leukemic leukocytes. Plasma suspensions of leukocytes obtained after fibrinogen sedimentation were studied in manometer vessels for 3 hours. Lymphocytes were characterized by high respiratory rate $\left(Q_{0_{2}}\right.$ ca 5$)$, high anaerobic glycolysis $\left(Q_{N_{2}}{ }^{4}\right.$ ca 25$)$, and virtually absent aerobic glycolysis, indicating a strong Pasteur mechanism. Granulocytes have a slightly lower respiration ( $\mathrm{Q}_{\mathrm{O}_{2}}$ ca 4 ), high aerobic glycolysis ( $\left.\mathrm{Q}_{\mathrm{O}_{2}}{ }^{\mathrm{A}} \mathrm{ca} 10\right)$, and high anaerobic glycolysis $\left(Q_{\mathrm{N}_{2}}{ }^{\wedge}\right.$ ca 25$)$

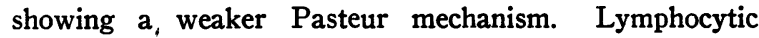
respiration was not influenced by the presence or absence of glucose, whereas glucose metabolism inhibited granulocyte respiration. These different regulatory mechanisms carry over to lymphoblasts and myeloblasts and are helpful laboratory aids in differentiating types of acute leukemia. High energy phosphate production (Seits method) was determined in leukocytes under varying metabolic conditions. There was no inhibition of ATP-ADP when normal and leukemic lymphocytes and granulocytes were incubated in glucose-free plasma for 3 hours. There was an average of $14 \%$ inhibition ( 0 to $47 \%$ ) when leukocytes were incubated under anaerobic conditions, with no detectable differences between lymphocytes and granulocytes. Combined anaerobiosis and glucose deprivation caused total ATP-ADP inhibition. These studies demonstrate the remarkable adaptability of lymphocytes as well as granulocytes to anaerobiosis or glucose deprivation despite their metabolic differences under "normal" conditions. Preliminary subcellular leukocyte studies indicate that these metabolic regulatory mechanisms may operate at the mitochondrial level. 
The Kinetics of Renal Urate Transport. WILloughBy Lathem* and Gerald P. Rodnan, Pittsburgh, Pa.

An analysis of the kinetics of renal urate transport under conditions of intravenous urate loading was made in normal subjects and in patients with primary gout. At increasing filtered loads, urate excretion rose in a curvilinear manner although, as previously reported, gouty subjects excreted less urate at corresponding loads than did normal subjects. The rate of reabsorption assumed as the difference between load and excretion, a curvilinear reabsorptive curve (reabsorption plotted against load) was found in gouty subjects. This curve had the following expression, which is consistent with a carrier-substrate reabsorptive process: $T=T_{\max } C /$ $\left(K_{t}+C\right)$, where $T=$ reabsorptive rate, $T_{\max }=$ maximal reabsorptive rate, $C=$ urate concentration in filtrate, and $K_{t}=$ a constant. When corrected for changes in initial (filtrate) concentration: $F=(1 / f) \ln [1 /(1-f)]$, where $F=$ correction factor and $f=$ the fraction of urate reabsorbed, $T_{\max }$ averaged $30.0 \mathrm{mg}$ per minute and $K_{t}$ $7.0 \mathrm{mg}$ per $100 \mathrm{ml}$. In contrast to gouty subjects the reabsorptive curve in normal subjects appeared to involve two rate-limiting steps: one governing reabsorption at rates below $\mathrm{Tm}$, the other that determining $\mathrm{Tm}$. The first of these was qualitatively similar to that found in gout, but $T_{\max }$ and $K_{t}$ were less, averaging $16.5 \mathrm{mg}$ per minute and $4.4 \mathrm{mg}$ per $100 \mathrm{ml}$, respectively. The administration of uricosuric agents altered the characteristics of the curves in both normal and gouty subjects. In normal subjects reabsorption was linear rather than curvilinear. This response, which argues against a secretory mechanism, is consistent with complete inhibition of active reabsorption and suggests reabsorption under these conditions by back diffusion. In gouty subjects reabsorption remained curvilinear, suggesting partial rather than complete inhibition of active reabsorption, and a $\mathrm{Tm}$, not ordinarily found, was demonstrated. These results indicate that at least two ratelimiting steps are involved in urate reabsorption and that uricosuric agents inhibit both.

Cytoplasmic Localization of Macroglobulin in Lymphocytoid Marrow Cells in Macroglobulinemia of Waldenström. Philip Leder AND MUHARREM GöKCEN, Minneapolis, Minn. (introduced by C. J. Watson).

Small, lymphocytoid round cells have been repeatedly observed in the bone marrow of patients with macroglobulinemia of Waldenström. This cell-type has been thought to be associated with the production of serum macroglobulin, but previous reports have failed to produce consistent results regarding the localization of cellular macroglobulin antigenic activity. To resolve this difficulty, antiserum to purified macroglobulin, isolated from a patient with macroglobulinemia of Waldenström, was produced and labeled with fluorescein isothiocyanate and utilized according to Coons' technique. The antiserum was completely adsorbed with purified human $\gamma$-globulin, removing cross-reacting components and leaving specific antimacroglobulin activity. Fixed, concentrated bone marrow smears obtained from a macroglobulinemic patient were incubated with labeled antimacroglobulin serum, both with and without previous blocking incubation with unlabeled antiserum. Others were incubated with labeled antihuman $\gamma$-globulin. DLpenicillamine-treated smears were also incubated with labeled antimacroglobulin. Striking fluoresence was noted in the cytoplasm of the lymphocytoid cells, both in the antimacroglobulin and antihuman $\gamma$-globulin-incubated smears, but most prominently in the former. Previous incubation with unlabeled antimacroglobulin effectively blocked the uptake of the labeled antimacroglobulin, confirming the specificity of the reaction. DL-penicillamine destroyed the antigenic property of the cytoplasmic macroglobulin in accordance with the anticipated effect on the macromolecule. Bone marrow smears from two additional patients with macroglobulinemia were also incubated with labeled antimacroglobulin and also showed (weak) cytoplasmic fluoresence of the lymphocytoid cells. Thus, the cytoplasm of the lymphocytoid cell probably represents storage, and possibly the site of synthesis, of this protein. In addition, the macroglobulinemic serum was tested for the presence of antinuclear and anticytoplasmic factors, with the use of peripheral blood, liver, and spleen as antigenic sources, and no activity was found.

The Effect of Osmolality and Sodium Concentration on the Metabolism of Glucose-U-C $C^{16}$ by Rabbit Kidney Cortex and Medulla In Vitro. James B. LeE, Vernon K. Vance and George F. Cahill, JR.,* Boston, Mass.

Establishment of osmotic gradients in kidney tissue appears fundamental to certain solute and water movements. Since maintenance of gradients requires energy, the present study was designed to investigate individual effects of osmolality and sodium chloride on glucose metabolism in cortex and medulla. Slices of kidney cortex and medulla of rabbit were incubated for 90 minutes at $38^{\circ} \mathrm{C}$ in Krebs-Ringer- $\mathrm{HCO}_{3}^{-}$buffer containing glucose-U-C ${ }^{14}, 10 \mathrm{mM}$. Variations in osmolality of the media were obtained by adding $\mathrm{NaCl}$, sucrose, or urea to $\mathrm{NaCl}$-free buffer. Measurements were made of labeled glucose incorporation into $\mathrm{CO}_{2}$, glycogen, and fatty acids, as well as of lactate production, glucose disappearance, and levels of glycogen and fatty acids. When medium osmolality was changed with sodium chloride or sucrose, maximal cortical glucose oxidation, disappearance, and lactate production occurred between 67 and $171 \mathrm{mOsm}$ per $\mathrm{kg} \mathrm{H}_{2} \mathrm{O}$. Further osmolality increases resulted in progressive inhibition of glucose metabolism to $50 \%$ of maximum at $360 \mathrm{mOsm}$ per $\mathrm{kg}$ $\mathrm{H}_{2} \mathrm{O}$. A secondary rise in glucose oxidation occurred at $550 \mathrm{mOsm}$ per $\mathrm{kg} \mathrm{H}_{2} \mathrm{O}$ before marked inhibition at $1,000 \mathrm{mOsm}$ per $\mathrm{kg} \mathrm{H}_{2} \mathrm{O}$. In contrast, glucose oxidation by medulla was unchanged even at $1,000 \mathrm{mOsm}$ per $\mathrm{kg}$ $\mathrm{H}_{2} \mathrm{O}$. Urea-induced changes in osmolality had little effect on cortical or medullary metabolism. With medium osmolality constant (281 mOsm per $\mathrm{kg} \mathrm{H}_{2} \mathrm{O}$ ), 
$\left[\mathrm{Na}^{+}\right]$was increased from 0 to 80 mmoles per $\mathrm{L}$, producing a rise in glucose oxidation by cortex, with characteristics of a first-order reaction (half maximal glucose oxidation at $\left[\mathrm{Na}^{+}\right]$of $25 \mathrm{mEq}$ per $\mathrm{L}$ ). In medulla there was no effect on glucose oxidation by $\left[\mathrm{Na}^{+}\right]$, although increasing $\left[\mathrm{K}^{+}\right]$depressed medullary glucose metabolism significantly. These results suggest that both "effective" extracellular osmolality and sodium concentration exert important independent effects on glucose oxidation in kidney cortex, but not medulla.

Reticuloendothelial Clearance of Circulating Fibrin in the Pathogenesis of the Generalized Shwartzman Reaction. Leung LeE, New York, N. Y. (introduced by Chandler A. Stetson).

The formation of fibrin polymers in the circulating blood during sublethal intravenous infusions of thrombin or thromboplastin in normal animals usually produces no detrimental effects, since the fibrin formed rarely accumulates in massive deposits in the peripheral vascular bed. The protective mechanism involved in the removal of fibrin in these circumstances has generally been considered to be the fibrinolytic system, and in man and some other species active fibrinolysis may indeed account for the uncommon occurrence of vasculoocclusive lesions complicating generalized coagulation disturbances. It has been found, however, that the reticuloendothelial system of the liver and spleen may also function as an efficient homeostatic mechanism in the clearance of circulating fibrin polymers and may be of particular significance in the rabbit in which active fibrinolysis during intravascular clotting cannot be demonstrated. This concept is supported by the finding in rabbits that interference with the phagocytic activity of the reticuloendothelial system during continuous intravenous thrombin infusions results in massive fibrin deposition in glomerular capillaries with ensuing bilateral renal cortical necrosis. The kidneys and other visceral organs exhibit intravascular "fibrinoid" and tissue necrosis indistinguishable from the changes considered pathognomonic of the generalized Shwartzman reaction elicited by bacterial endotoxins. Furthermore, several experimental situations have promoted fibrin accumulation after either a single injection of endotoxin or an infusion of thrombin, indicating that similar mechanisms are involved in the action of these two substances. From these and other experiments to be described, it is concluded that bacterial endotoxins are potent activators of the coagulation mechanism and that the generalized Shwartzman reaction in the rabbit results from the failure of the reticuloendothelial system to adequately clear the circulation of fibrin formed during intravascular coagulation.

Observations on Hepatic DNA Synthesis in Man. CARRoll M. Leevy, Jersey City, N. J. (introduced by Harold Jeghers).

An in vitro radioautographic technique was used to investigate DNA synthesis in percutaneous liver biopsies from 38 normal subjects and 48 patients with liver disease (17 fatty liver, 5 viral hepatitis, 4 metastatic malignancy, 4 postnecrotic cirrhosis, 18 Laennec's cirrhosis). Serial studies were obtained in selected subjects and the results correlated with clinical findings, liver function tests, and routine histopathological studies. Specimens immersed in $3 \mathrm{ml}$ of Ringer's solution containing $50 \mu \mathrm{c}$ of tritiated thymidine (specific activity 1.9) and $75 \mathrm{U}$ of hyaluronidase were incubated with constant agitation at $37^{\circ} \mathrm{C}$ for 1 hour in a $5 \% \mathrm{CO}_{2}, 95 \% \mathrm{O}_{2}$ atmosphere. Radioautographs were prepared from paraffin sections with $\mathrm{NTB}_{3}$ emulsion and Kodak D-19 developer and standard techniques. Preliminary rat studies demonstrated that in vitro labeling was equivalent to that obtained in vivo. Specificity of labeling was confirmed with DNAase. Temperature, $\mathrm{pH}$, and length of incubation influenced labeling; addition of ATP and colchicine had no effect. Normal liver and uncomplicated fatty liver were characterized by approximately 1 labeled cell per 10,000 cells; a maximal increase in labeling of 250 -fold was noted in malignancy, 50 -fold in postnecrotic cirrhosis, 20-fold in acute viral hepatitis, and 10-fold in Laennec's cirrhosis. DNA synthesis was related to the degree of necrosis and inflammation, persisting with activity in acute lesions and returning to normal in inactive cirrhosis. In Laennec's and postnecrotic cirrhosis increased labeling was noted in both parenchymal and connective tissue cells. These results establish the feasibility of assessing hepatic DNA synthesis in man, demonstrate that both connective tissue proliferation and parenchymal cell regeneration contribute to development of human cirrhosis, and provide a baseline for evaluating effects of medicinal agents.

A Comparison of Direct-current and Alternating-current Defibrillation, under Conditions of Hypothermia. Armand A. Lefemine, Raghavan Amarasingham, Dwight E. Harken, Barough Berkovitz and BerNARD Lown, Boston, Mass. (introduced by Fredrick Stare).

Ventricular fibrillation is a frequent occurrence during open-heart operations. The use of alternating current (AC) for reversion may require multiple shocks that are associated with myocardial damage. At times defibrillation is impossible. A direct-current (DC) defibrillator has been consistently effective in defibrillating the normothermic animal across the intact chest. To evaluate DC defibrillation in closed-chest dogs, comparative studies with AC were carried out at esophageal temperature levels ranging from $37^{\circ}$ to $20^{\circ} \mathrm{C}$. Cooling was achieved by an extracorporeal system. Ventricular fibrillation was induced by single monophasic capacitor discharges synchronized to occur during the vulnerable phase of the ventricular cycle. Both types of countershock were compared under analogous conditions of temperature, $\mathrm{pH}, \mathrm{PCO}_{2}$, and electrolyte concentrations. DC defibrillation was tried in 143 episodes and was successful in all but $3(2 \%)$; these failures were noted in a single animal at $20^{\circ} \mathrm{C}$. A total of $258 \mathrm{DC}$ shocks 
was required, of which $154(60 \%)$ were effective. The cnergy levels ranged from 40 to 400 watt-seconds. AC defibrillation was tried in 91 episodes and was unsuccessful in $16(18 \%)$. A total of 267 shocks was given of which $77(29 \%)$ were successful. Voltages in the range of 500 to 1,000 were employed. AC defibrilllation became increasingly difficult as the esophageal temperature fell below $30^{\circ} \mathrm{C}$ and was followed frequently by atrial and ventricular arrhythmias. Such arrhythmias rarely followed DC countershock. Subcutaneous and intramyocardial temperatures were recorded during countershock. Rises of 2 to $4^{\circ} \mathrm{C}$ occurred after single AC discharges. No temperature changes followed the use of DC. Three clinical experiences during openheart surgery confirmed the superiority of DC observed experimentally. The first case was defibrillated readily with $\mathrm{DC}$ when ten $\mathrm{AC}$ attempts had failed. Alternating current defibrillation has been abandoned for use in the operating room.

Infections with Reovirus Type 2. A. MARTIn LeRner, James D. Cherry, Jerome O. Klein and Maxwell Finland,* Boston, Mass.

In the course of viral studies of febrile respiratory and exanthematous illnesses, rises in hemagglutinininhibiting antibodies (HIA) to type 2 reovirus were demonstrated in eight children observed in 1960 ; seven of them had a morbilliform rash but most of them had no respiratory or enteric symptoms. The virus was isolated from three patients-four times during the first 4 days of illness in one of them. Among matched controls without illness or in children without illnesses, only one yielded this virus. Constantly elevated or falling titers of HIA to type 2 reovirus were demonstrated in occasional asymptomatic children and in more than one-fifth of those with exanthems or lower respiratory infections considered to be due to other agents. In three of the patients with reovirus type 2 infection, the HIA titers obtained with prototype strains of types 1 and 3 reovirus were also elevated in sera obtained during the first weeks after infection, but only the type 2 HIA remained elevated in sera obtained 1 year later. About one-fourth of a small group of patients studied in 1959 and of similar ones observed in 1961 were found to have HIA to reovirus type 2. About one-third of a larger number of patients studied over the 3-year period has serological evidence of infection with reoviruses, approximately one-half of them due to type 2 . The evidence suggested placental transmission with loss of antibody within the first 6 months and reacquisition in about half of the children before 10 years of age. The data also suggest that the type 2 reovirus may be associated with a rubelliform exanthem.

The Mechanism of Intestinal Absorption of Bilirubin. Roger Lester and Rudi Schmid,* Boston, Mass.

It has been shown recently that, in rats, intraduodenal administration of either free or conjugated $\mathrm{C}^{\mathbf{1 4}}$-bilirubin results in biliary re-excretion of conjugated labeled pig- ment corresponding to 10 to $30 \%$ of the administered dose. This indicates that bilirubin undergoes a significant enterohepatic circulation. The present studies were performed to determine whether both free and conjugated pigments are absorbed from the gastrointestinal tract unaltered, and whether absorption occurs in humans. Congenitally jaundiced (Gunn) rats retain free bilirubin but excrete intravenously administered conjugated bilirubin in bile. In Gunn rats, intraduodenal instillation of either free or conjugated $\mathrm{C}^{\mathbf{1 4}}$-bilirubin resulted in significant radioactivity in free bilirubin crystallized from serum. Moreover, after intraduodenal instillation of conjugated $\mathrm{C}^{\mathbf{4}}$-bilirubin, virtually no radioactive bilirubin was recovered in bile. Estimates of the specific activity and total miscible pool of free bilirubin revealed that total intestinal absorption approximated $15 \%$ of the dose irrespective of whether free or conjugated pigment was administered. One and one-half $\mu \mathrm{c}$ of free $\mathrm{C}^{14}$-bilirubin was administered by duodenal tube to a child with congenital unconjugated hyperbilirubinemia. From the specific activity of the serum bilirubin and the total miscible bilirubin pool, it was estimated that approximately $80 \%$ of the administered dose was absorbed and retained by the subject at 24 hours. A similar experiment was performed in a mildly icteric woman in whom external biliary drainage had been instituted; $15 \%$ of the intraduodenally administered free $\mathrm{C}^{\mathbf{1 4}}$-bilirubin appeared as conjugated $\mathrm{C}^{\mathbf{1 4}}$-bilirubin in bile collected over an 8-hour period. These studies indicate that free bilirubin is absorbed by the gastrointestinal tract of rats and humans. In contrast, little if any conjugated bilirubin is absorbed unaltered, but a significant fraction is hydrolyzed and absorbed as free bilirubin.

Analog Computer Analysis of Diffusing Capacity Measurements. B. M. Lewis,* P. K. AdhikARI, S. F. Boushy and A. Sakamoto, Detroit, Mich.

This work is an attempt to apply modern techniques of high-speed computing to the determination of diffusing capacities of regions of the lung and from this to assess the accuracy with which over-all diffusing capacity can be measured by methods currently in use. Since the accuracy of such methods is disputed in emphysema, patients with this disease were used as subjects for this study. These patients rebreathed a mixture of neon and carbon monoxide in air for a 45-second period. Neon concentration was analyzed continuously by a mass spectrometer, and the contents of the rebreathing bag were circulated through an infrared $\mathrm{CO}$ analyzer. Tidal volume was measured with a pneumotachograph. Residual volume and dead space were measured indeendently. An analog computer was used to simulate a lung of two regions and a rebreathing bag. The residual volume and tidal volume of each region were then varied until the mixing curve of the simulated lung matched the mixing curve of neon in the patient. A simulated diffusing capacity was then added to each region and these regional diffusing capacities altered until 
the patient's $\mathrm{CO}$ absorption curve was matched. In two patients with slow mixing and a low diffusing capacity calculated from the $\mathrm{CO}$ absorption curve ("calculated" $\mathrm{DL}_{\mathrm{L}}$ ), the sum of the diffusing capacities of the two regions ("true" DL) agreed closely with the "calculated" DL. In two others with slow mixing and a normal calculated DL, "true" DL exceeded calculated DL. In one patient with moderate impairment of mixing and a normal calculated DL, true DL was slightly lower than calculated DL.

Effects of Ethanol Metabolism on Serum Concentration and Urinary Excretion of Urate in Man. ChARLES S. Lieber, Don P. Jones, Monty S. Losowsky and Charles S. Davidson,* Boston, Mass.

The traditional association between gout and alcoholism led us to test whether a relationship exists between ethanol and urate metabolism. Serum urate was measured by the uricase method in 12 acutely intoxicated subjects. In 5 , initial concentrations varied from 9.7 to $11.5 \mathrm{mg}$ per $100 \mathrm{ml}$, while in the others they were below the upper limit of normal (7.5). Urate concentrations decreased in each case to below $6.8 \mathrm{mg}$ per $100 \mathrm{ml}$ during recovery. The average decrease was $38 \%(\mathrm{p}<0.001)$. Ethanol administration (intravenous, 2 subjects, 8 to 12 hours; oral, 5 subjects, 8 to 72 hours) was accompanied in each instance by a rise in serum urate ( 1 to $3.2 \mathrm{mg} / 100 \mathrm{ml}$ ), which returned to normal after omission of ethanol. Blood ethanol concentrations reached 200 to $340 \mathrm{mg}$ per $100 \mathrm{ml}$. Glucose in isocaloric amounts (oral) or saline (intravenous) given to two of these subjects did not change serum urate concentrations. In three instances in which urinary studies were performed, clearances and outputs of urate decreased by 50 to $85 \%$ during 24 hours of ethanol administration, and returned to control values thereafter, while creatinine clearances remained unchanged. This deficit in urate output could account for the observed rise in serum urate. During ethanol administration, blood lactate rose to 16 to $23 \mathrm{mg}$ per $100 \mathrm{ml}$; and similar levels were found in all 8 acutely intoxicated subjects (blood ethanol $311 \pm 16.8 \mathrm{mg} / 100 \mathrm{ml}$ ) in whom such measurements were made. Sodium lactate ingestion on two occasions by one of the subjects formerly given ethanol achieved blood lactate concentrations, decreases in urate excretion, and increases in serum urate similar to those after ethanol, while urinary $\mathrm{pH}$ changed in the opposite direction. Conclusions: 1) high serum urate levels occur in acutely intoxicated subjects; 2) alchohol increases serum urate; 3 ) this effect is mediated through a rise in blood lactate that decreases urinary urate.

The Regulation of Intragastric Hydrogen Ion Concentration: Evidence Regarding a Na-H Exchange Mechanism in Man. ARthur E. Lindner and NaTHANIEl Cohen, New York, N. Y. (introduced by Henry D. Janowitz).

Intragastric mechanisms that regulate $\mathrm{H}^{+}$concentration were studied by instilling several isotonic hydrogen- containing solutions and a dilution-indicator system into the stomachs of 17 human subjects. Solution I contained $\mathrm{H}^{+}$and $\mathrm{Cl}^{-}(160 \mathrm{mEq} / \mathrm{L})$. Solution II contained $\mathrm{H}^{+}$ $(100 \mathrm{mEq} / \mathrm{L}), \mathrm{Na}^{+}(60 \mathrm{mEq} / \mathrm{L})$ and $\mathrm{Cl}^{-}(160 \mathrm{mEq} / \mathrm{L})$. Osmolarity and electrolyte concentrations were determined in samples recovered at 15-minute intervals over 1 -hour periods. With the aid of the dilution indicator (phenol red) the volume of fluid added by secretion as well as that lost through the pylorus by emptying could easily be calculated. Similar results were obtained with both solutions. In all experiments $\mathrm{H}^{+}$declined linearly with time. The fall in hydrogen concentration was associated with a parallel decline in osmolarity and with a reciprocal (straight line) rise in sodium concentration. Potassium in increasing concentrations entered the gastric fluid progressively. $\mathrm{Cl}^{-}$concentration declined moderately. The electrolyte composition of the fluid entering and leaving the stomach was calculated from the observed concentrations. The results thus obtained suggest that several mechanisms other than emptying are responsible for the lowering of $\mathrm{H}^{+}$ concentration of instilled (and presumably secreted) acid: 1) entry of a fluid free of $\mathrm{H}^{+}$and containing $\mathrm{HCO}_{3}^{-}$ (so-called alkaline component) with consequent fall in osmolarity by evolution of $\mathrm{CO}_{2}$; and 2) an exchange mechanism ( $\mathrm{Na}$ for $\mathrm{H}$ ) is required to account completely for the observed reduction in $\mathrm{H}^{+}$concentration in nearly one-third of the experimental periods. The entry of $\mathrm{K}$ (as in our previously reported studies) is again best correlated with the rate of gastric secretion.

Cell Proliferation Kinetics in Human Gastrointestinal Mucosa. Martin Lipkin, Paul Sherlock and BerTRAND BeLl, New York, N. Y. (introduced by Thomas P. Almy).

Injection of tritiated thymidine into four human subjects has provided a means of studying the kinetics of cell proliferation and migration in normal and abnormal gastrointestinal mucosa. Microradioautographs made from mucosal biopsies of injected patients have been analyzed to measure the total duration and component portions of the mitotic cycle of the epithelial cells, as well as the rate of migration and removal of the cells. In normal mucosa of the transverse colon, the percentage of labeled epithelial cells in mitosis reaches a peak at 6 to 8 hours. The mean generation time of the epithelial cells is 22 hours. The duration of the DNA synthesis (S) phase is 15 hours, and the premitosis $\left(G_{2}\right)$ and mitosis (M) phases together comprise about 2 hours. After administration of 5-fluorouracil, abnormalities in chromatin pattern and size of nuclei are seen. Incorporation of thymidine is rapid, but the durations of the $G_{2}$ and $M$ phases are lengthened to 4 hours, and the peak of labeled mitoses is delayed. In a rectal carcinoma, earlier and heavier labeling is noted in tumor cells compared with adjacent normal epithelial cells. In the stomach, earliest labeling occurs in the depths of the gastric glands, whence labeled cells migrate to the surface in 48 hours. Labeling is less marked in zymogen cells; 
none was observed in parietal cells. Some epithelial cells in colon and stomach incorporate thymidine but do not proliferate rapidly, and are retained in the mucosa for prolonged periods. These findings correspond closely to kinetics of proliferation previously observed in the mouse and rat and provide a basis for study of disease states in man.

The Effects of Alcohol in Experimental Infections in Mice. Donald B. Louria, New York, N. Y. (introduced by Robert F. Watson).

Certain bacterial infections occur with inordinate frequency in alcoholics. In the present studies, mice were given $0.5 \mathrm{ml}$ of ethyl alcohol subcutaneously one-half hour prior to infection. After intravenous inoculation with staphylococci, 18 of 81 controls (22\%) died in 14 days, as did 93 of $142(65 \%)$ which became ataxic but not stuporous after alcohol, and 88 of $112(81 \%)$ of those developing coma. Kidney staphylococcal populations were similar in all groups at 2,4 , and 6 hours but were significantly higher in alcohol-treated comatose or ataxic mice 24 hours after infection. Peritoneal clearance of Escherichia coli and staphylococci was normal in alcohol-treated mice if small numbers of microorganisms were introduced intraperitoneally, but with larger inocula, clearance was markedly reduced. Populations were similar in all groups during the first hour, but 2 to 6 hours after infection the microbial census fell over $95 \%$ in $74 \%$ of controls, in $35 \%$ of ataxic mice, and in $13 \%$ of those that were stuporous. Quantitative measurement of the polymorphonuclear response showed that 2 hours after infection only small numbers of polymorphonuclear leukocytes entered the peritoneal cavity of alcohol-treated mice and 4 hours after infection, polymorphonuclear counts averaged $58 \%$ less in ataxic animals, and $88 \%$ less in comatose mice, than in controls. Since results were similar in ataxic and comatose animals, coma, hypotension, and shock cannot be implicated in these experiments. If peritoneal inflammation was produced by injection of starch-aleuronat prior to infection, alcohol administration did not alter phagocytosis or intracellular killing significantly, suggesting that the enhancement of infection observed in these studies was related primarily to delay in polymorphonuclear inobilization.

The Use of Synchronized Direct-current Countershock in the Treatment of Cardiac Arrhythmias. BERNARD Lown, Raghavan Amarasingham, Jose Neuman AND Barouh Berkovitz, Boston, Mass. (introduced by Samuel A. Levine).

Some cardiac arrhythmias are refractory to all forms of treatment. In principle, external electric countershock that can induce complete depolarization of the heart and permit resumption of sinus activity would be an ideal form of treatment if it were safe. Experiments were conducted to determine what form of electrical stimulation would be most safe and effective. Nineteen dogs in sinus rhythm received a total of 438 alternating-current countershocks (AC) across the closed chest, with voltages effective for ventricular defibrillation and with lower levels. There was a high incidence of diverse arrhythmias including 78 episodes of ventricular fibrillation $(18 \%)$. Seven animals died during the first week after countershock. AC countershock was therefore considered unsafe for elective use in patients. A direct-current defibrillator (DC) delivering a brief monophasic discharge was developed. In 30 dogs receiving $858 \mathrm{DC}$ countershocks there was a $1.0 \%$ incidence of ventricular fibrillation and no deaths. This technique was consistently effective in defibrillating the heart even when $\mathrm{AC}$ failed. In the use of $\mathrm{DC}$, ventricular fibrillation occurred only when the discharge fell during a predictable brief vulnerable period of the ventricular cycle. To prevent this arrhythmia entirely, a synchronizer was developed which permitted the shock to be delivered outside this zone. Thus in 2,600 synchronized shocks, ventricular fibrillation never resulted unless the shock was triggered within the vulnerable period. Single synchronized DC shocks were applied across the closed chest in two patients with ventricular tachycardia resistant to all therapy and four patients with long-standing atrial fibrillation. Reversion was instantly achieved in all except one with atrial fibrillation. There were no complications. This extensive experimental and limited clinical experience suggests that a new simple and effective method of controlling some serious arrhythmias may be available.

Hyponatremia in Acute Porphyria Probably Due to Inappropriate Secretion of Antidiuretic Hormone. GEORGE D. Ludwig* and Martin Goldberg, Philadelphia, Pa.

In the past, it has been noted that hyponatremia frequently accompanies acute attacks of porphyria, but the cause has never been adequately explained. Eight patients displaying the syndrome of hyponatremia, serum hypo-osmolality, natriuresis, and urine hypertonicity were studied. Serum sodium and osmolality were as low as $100 \mathrm{mEq}$ per $\mathrm{L}$ and $220 \mathrm{mOsm}$ per $\mathrm{kg}$, respectively, in some cases. Edema, azotemia, and dehydration were absent. The syndrome probably results from inappropriate secretion of ADH. Adrenal insufficiency, chronic renal disease, and "true" sodium depletion were excluded. Central neurological involvement, such as coma, convulsions, or acute psychosis, was correlated with the most severe changes in serum sodium and osmolality. In selected patients, restriction of fluids to 800 to 1,000 $\mathrm{ml}$ per day resulted in dramatic clinical improvement with reversal of serum sodium and osmolality toward normal. In one of these patients, increasing fluid intake to 3,000 $\mathrm{ml}$ per day produced a sharp fall in serum sodium and osmolality that was accompanied by a convulsive seizure. With subsidence of acute attacks in these patients, the initial greatly increased excretion of porphyrins and porphyrin-precursors decreased as normal values for serum sodium and osmolality were achieved, and the urine became hypotonic. Oral water loading then failed to reproduce the hyponatremic syndrome. This syn- 
drome provides an explanation for the oliguria and possibly some of the central neurological effects of acute porphyria. The syndrome apparently results from central effects of porphyria, although exogenous nonosmotic stimuli of $\mathrm{ADH}$ secretion, such as barbiturates, analgesics, trauma, and dimercaprol, might have contributed in some instances. A new approach to therapy of acute attacks is suggested which differs radically from that previously recommended, since dimercaprol and EDTA appear to be contraindicated.

The Effects of Leukocyte Respiration in Leukemia. Harold J. Lynch, Jr. and John Laszlo, Durham, N. C. (introduced by Albert Heyman).

The increased oxygen consumption observed in patients with leukemia is thought to reflect increased leukocyte metabolism. This report describes a simple method for measuring $\mathrm{O}_{2}$ uptake, $\mathrm{CO}_{2}$ and lactic acid production, and $\mathrm{pH}$ changes in blood resulting from leukocyte metabolism. Repeated observations were made on normal controls, patients with leukocytosis of infection, and 12 subjects with myelogenous leukemia. Arterial blood was collected in a sealed syringe and kept at $37^{\circ} \mathrm{C}$. At 5- to 15-minute intervals blood $\mathrm{O}_{2}$ content, $\mathrm{PCO}_{2}$, lactic acid content, and $\mathrm{pH}$ were determined on aliquots of the blood. Total body $\mathrm{O}_{2}$ consumption ( $\mathrm{TBC} \mathrm{O}_{2}$ ) and tolerance to local ischemia were also determined. In patients with leukemia, the blood $\mathrm{O}_{2}$ content fell rapidly (mean, $0.14 \mathrm{ml} / 100 \mathrm{ml} / \mathrm{min}$ in blood containing $>$ 100,000 white blood cells $(\mathrm{WBC}) / \mathrm{mm}^{8}$ ). This fall was blocked by cyanide and by cooling to $2^{\circ} \mathrm{C}$. The $\mathrm{O}_{2}$ content of controls fell less rapidly. The mean $\mathrm{O}_{2}$ uptake was $56 \mu 1$ per $10^{10}$ WBC per minute (range, 23 to 87 ) in both leukemic and normal blood. Lactic acid content increased (as much as $1 \mathrm{mg} / 100 \mathrm{ml} / \mathrm{min}$ ) while $\mathrm{pH}$ fell rapidly and $\mathrm{PCO}_{2}$ rose slightly in blood containing $>100,000 \mathrm{WBC}$ per $\mathrm{mm}^{3}$. The mean $\mathrm{TBC} \mathrm{O}_{2}$ of the leukemic patients was $212 \mathrm{ml}$ per minute per $\mathrm{m}^{2}$ (normal 120 to 160 ). Respiratory alkalosis was observed in all leukemic subjects as indicated by mean values for $\mathrm{pH}$ and $\mathrm{PCO}_{2}$ of 7.48 and $29.3 \mathrm{~mm} \mathrm{Hg}$, but severe acidosis developed in some patients terminally. By estimating whole body leukocyte populations in patients with chronic granulocytic leukemia, the observed $\mathrm{O}_{2}$ uptake of leukocytes could account for 17 to $50 \%$ of the oxygen consumption of the total body. Decreased tolerance to ischemia was observed in the leukemics, suggesting that relative hypoxia may occur in tissues, owing to competition with leukocytes for oxygen, especially in areas with slowed circulation. The method described is useful for measuring leukocyte metabolism under approximately in vivo conditions.

Disappearance Rates of Estrone, Estradiol-17 $\beta$, and Estriol from Plasma and Urine after Delivery of the Placenta in the Human. F. Debele Maner and John R. K. Preedy,* Atlanta, Ga.

Although the levels of urinary estrogens in the postpartum period have been estimated, observations have been sparse. Plasma estrugens during this period have been little studied, and no correlation has been established between plasma and urine levels. Accordingly a study was carried out as follows. Plasma samples were drawn from normal female subjects at the time of delivery of the placenta, and at intervals thereafter, together with 4-, 8-, 12-, and 24-hour urine collections. Plasma and urine samples were analyzed for total estrone, estradiol-17 $\beta$, and estriol content. Estradiol-17 $\beta$ disappeared from plasma to levels below the sensitivity of the method at a single rapid rate $\left(t_{1} \approx 0.8 \mathrm{hrs}\right)$, whereas estrone and estriol each disappeared at two well defined rates, a faster $\left(t_{\frac{1}{2}} \approx 2.0 \mathrm{hrs}\right.$ for estrone, $\mathrm{t}_{1} \approx 1.9 \mathrm{hrs}$ for estriol) for 3 to 5 hours, followed by a slower $\left(t_{1} \approx 16.3 \mathrm{hrs}\right.$ for estrone, $t_{\frac{1}{3}} \approx 25.4 \mathrm{hrs}$ for estriol) for longer than 45 hours. Estrone, estradiol$17 \beta$, and estriol each disappeared from the urine at two rates, a faster ( $t_{\frac{1}{1}} \approx 3.1,3.06,3.45 \mathrm{hrs}$, respectively) for 5 to 10 hours, followed by a slower $\left(t_{1} \approx 22.9,22.9\right.$, $14.5 \mathrm{hrs}$, respectively) for more than 80 hours. Since the transfer of estrogens from the placenta to the maternal circulation may be regarded as a long-continued infusion, it is reasonable to assume that all pools of distribution are in approximate equilibrium. The two rates of disappearance of both estrone and estriol from plasma could be accounted for in part by the presence of rapidly and slowly excreted components of the total plasma estrone and estriol, respectively. This possibility is supported by the finding that, in the case of estriol, the renal clearance during the slow disappearance phase is less than half that during the rapid phase. Possible slow mobilization of estrogen from stores or compartments other than plasma may also affect the rate of disappearance.

Impairment of Cardiovascular Response to Tyramine by Reserpine Administration in Man. M. L. MASHFORD And W. A. Mahon, Boston, Mass. (introduced by Shu Chu Shen).

Reserpine has not been shown to deplete tissue norepinephrine in man, although this effect is well established in animals. Since tyramine produces its pressor effect by releasing endogenous norepinephrine at sympathetic nerve endings, the response to tyramine has been used to demonstrate that reserpine, in therapeutic doses, depletes tissue norepinephrine. Resting supine blood pressure was recorded continuously in 12 patients; $0.2 \mathrm{mg}$ per $\mathrm{kg}$ tyramine was given i.v. after the blood pressure had stabilized. Mean pressure was graphed for each patient against time, and the area enclosed between this curve and the preinjection level was the statistic used for comparison. In eight patients $0.03 \mathrm{mg}$ per $\mathrm{kg}$ reserpine was given i.v. after the first study, and again 24 hours later, and the observations were repeated 48 hours after the initial study. In five patients no reserpine was given. One patient was studied 3 times at 48-hour intervals; reserpine was given between the second and third studies, and he thus figures in both reserpinized and control groups. Cardiac outputs (indocyanine green) were performed at intervals 
before and after tyramine on both days in 10 of the 12 patients, and total peripheral resistance was calculated from these data. The pressor response to tyramine was significantly less on the second occasion in patients receiving reserpine (mean difference in area, -888 ; $p<0.01$ ) in contrast to the control patients whose response was not significantly different on the two occasions (mean difference in area, +84). Cardiac output changes after tyramine were variable and were not significantly different after reserpine. In the reserpinized patients the increase in total peripheral resistance produced by tyramine was significantly smaller on the second occasion (mean difference, 798 dynes- $\mathrm{cm}-\mathrm{sec}^{-5}$; $\mathrm{p}<0.025)$. Thus, reserpine has been shown to impair cardiovascular responses which are known to depend upon intact norepinephrine stores.

Pseudofeedback Inhibition of Purine Synthesis by 6-Mercaptopurine and Other Purine Analogues. ROBERT J. McCollister, Walter R. Gilbert, Jr. and James B. WyngaARden,* Durham, N. C.

6-Mercaptopurine exerts a wide spectrum of antimetabolic effects, but these are not fully understood. 6-MP is sparingly incorporated into DNA and RNA. 6-MP ribonucleotide is an inhibitor of reactions which convert inosinic acid to adenosine monophosphate and guanosine monophosphate. However, neither incorporation into "fraudulent" nucleic acids nor inhibition of reactions at the ribonucleotide level explains its inhibition of de novo synthesis of purines. We have now found that 6-MP ribonucleotide is a potent inhibitor of the first enzyme specifically concerned with purine synthesis, phosphoribosylpyrophosphate (PRPP)-amidotransferase, This enzyme catalyzes formation of phosphoribosylamine from PRPP and glutamine, and is normally the site of feedback regulation by adenyl ribonucleotides. ATP, $\mathrm{ADP}$, and AMP are competitive inhibitors of both PRPP and glutamine binding. 6-MP ribonucleotide in comparatively low concentrations mimics the action of adenyl ribonucleotides in a pseudofeedback inhibition of purine synthesis. $K_{1}$ (enzyme-6-MP ribonucleotide dissociation constant) is $5.3 \times 10^{-5} \mathrm{M}$, much lower than that of any normal ribonucleotide monophosphate inhibitor, and $1 / 5$ the $K_{m}$ of PRPP and $1 / 11$ the $K_{m}$ of glutamine (pigeon liver enzyme). The ribonucleotides of 6-thioguanine and 8-azaguanine are also effective inhibitors of PRPP-amidotransferase: $\mathrm{K}_{1}=1.8 \times 10^{-4} \mathrm{M}$ and $5.4 \times 10^{-4} \mathrm{M}$, respectively. (The ribonucleotides and free bases are noninhibitory.) Furthermore, in this system the inhibitory effects of these analogue ribonucleotides and of AMP are additive. Thus, the ribonucleosides of several purine analogues are potentially very effective regulators of purine synthesis de novo. These compounds may therefore have multiple points of action at which they exert their antimetabolic effects. The concept that ideal chemotherapeutic agents may be compounds which in their primary action mimic or augment normal intracellular regulators deserves further evaluation.
Remarkable Anticholinesterase Tolerance in Nonmyasthenic Patients. Michael P. McQuillen AND RICHARD J. Johns, Baltimore, Md. (introduced by Richard H. Shepard).

Five patients were studied who tolerated the daily administration of 15 to 75 times the usual toxic dose of anticholinesterase drug (neostigmine or pyridostigmine) without ill effect. In the past, such pronounced tolerance to these drugs was thought to be possible only in the presence of myasthenia gravis, and these patients were diagnosed as having myasthenia on the basis of this erroneous belief. However, electromyographic studies showed that this tolerance was not on the basis of myasthenia gravis, for these patients did not have a demonstrable defect in neuromuscular transmission. Nevertheless, their response to neostigmine was not normal, for neostigmine did not produce its characteristic block of neuromuscular transmission when administered to these patients. The patients exhibited no dependence upon the anticholinesterase drug on its abrupt withdrawal. Tolerance apparently developed with the prolonged administration of gradually increasing doses of anticholinesterase drug. A similar tolerance was produced in rats and mice by the repeated administration of sublethal doses of pyridostigmine or isofluorophate over a 5-week period. In the animal, tolerance to the direct effect of the drug on cholinesterase did not occur, for comparable blood cholinesterase inhibition occurred in tolerant and in nontolerant animals. Presumably the tolerance was developed to the excess acetylcholine itself.

The Hypoglycemic Effect of Ketone Bodies. David Mebane and Leonard L. Madison,* Dallas, Tex.

Although recent evidence suggests that ketores may inhibit glucose utilization by the rat diaphragm, the in vivo effects of hyperketonemia on carbohydrate metabolism have not been adequately characterized. The present study was designed to determine simultaneously the effect of infusions of $\beta$-hydroxybutyric acid $(\mathrm{BOH})$ and of acetoacetate (AA) on hepatic glucose output (HGO), peripheral glucose utilization (PGU), and arterial glucose concentration. Dogs with chronic endto-side portacaval shunts were used, thereby permitting measurement of hepatic rather than splanchnic glucose output. HGO [hepatic blood flow (Bradley) $\times$ hepatic vein-arterial glucose concentration difference] was measured at 10-minute intervals during the 40 -minute control period, and for 135 minutes during and after ketone infusions ( 5.5 to $10 \mathrm{mmoles} / \mathrm{kg} / \mathrm{hr}$ ). In all 12 studies both $\mathrm{BOH}$ and $\mathrm{AA}$ produced a significant hypoglycemic effect, arterial glucose concentration falling below 40 $\mathrm{mg}$ per $100 \mathrm{ml}$ in some studies. This hypoglycemia was entirely the consequence of a marked reduction in HGO. During the last 30 minutes of ketone infusion, HGO averaged only $24.5 \mathrm{mg}$ per minute, a $60 \%$ fall from the control value of $61 \mathrm{mg}$ per minute. The decrease in the glucose pool was not commensurate with the reduction in $\mathrm{HGO}$ as a consequence of a $40 \%$ decrease 
in PGU. These data indicate that induced hyperketonemia has a profound and immediate effect on glucose metabolism. Not only is PGU impaired but, as a result of a greater reduction in $\mathrm{HGO}$, hypoglycemia supervenes. Since the infusions of the ketone acids and their sodium salts, which resulted respectively in systemic acidosis

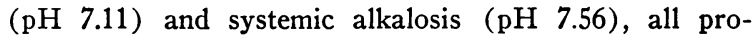
duced identical changes in carbohydrate metabolism, these changes can be ascribed to hyperketonemia per se and not to alterations in acid-base balance.

Studies on the Regulation of Iron Absorption in Experimental Hypoplastic and Iron-deficiency Anemias. Gerald A. Mendel and Robert J. Weiler, Chicago, I11. (introduced by Leon O. Jacobson).

In our previously reported studies utilizing splenectomy and radiostrontium administration to suppress erythropoiesis in mice, evidence was presented that the augmentation of iron absorption induced by atmospheric hypoxia can occur independently of acceleration of erythropoiesis. Confirmatory data have been obtained by exposing iron-deficient mice to reduced oxygen tension. Augmentation of iron absorption without measurable change in erythropoiesis (as assessed by histologic examination of the bone marrow, reticulocyte count, and measurement of the incorporation of intravenously administered iron into circulating red blood cells) was also found to occur in this preparation, thus verifying this observation. In order to evaluate whether anemic anoxia might play a role in the regulation of iron absorption, which is independent of body iron content and the rate of erythropoiesis, groups of splenectomized, radiostrontium-treated mice were further treated with phenylhydrazine. Enhancement of iron absorption was found to occur with the increased degree of anemia so produced. To determine the effect of reduction in the rate of erythropoiesis on iron absorption in iron-deficient mice, the effect of splenectomy and radiostrontium administration in such animals was studied. Iron absorption in splenectomized, radiostrontium-treated, iron-deficient mice remained at a level greater than four times normal, in spite of the marked suppression of erythropoiesis produced. In summary, these preliminary studies suggest that: 1) anemic anoxia per se may play a role in the regulation of iron absorption in hypoplastic anemia ; 2) iron-deficient animals continue to absorb iron in quantities several-fold greater than normal, in spite of substantial reduction in the rate of erythropoiesis produced by splenectomy and radiostrontium administration; and 3) the observation that the enhancing effect of hypoxia on iron absorption is independent of acceleration of erythropoiesis is confirmed.

The Effect of Cytotoxic Antibody In Vivo. Observations by a Nere Technique. John P. Merrill,* Colette Hanau and Donald E. Oken, Boston, Mass.

It is now clear that damage to cells through the action of cytotoxic antibody (CA) may occur before histologic evidence of this is present in fixed tissue sections. The action of CA on cell suspensions is characterized by cell swelling, suggesting inability of the cell to maintain osmolality of its internal environment either through change in permeability of cell membrane or through interference with metabolic processes necessary for the "sodium pump." In an effort to study this phenomenon in the intact metabolizing tissue we have utilized measurements of the short circuit current developed by the frog skin as a function of active transport of sodium. Rabbits immunized by the injection of minced frog skin develop antisera to frog tissues, as evidenced by a cytotoxic effect which causes rapid lysis of nucleated frog erythrocytes. These sera, when applied to either or both surfaces of the frog skin, with or without complement and spleen cells from the immune rabbit, show no effect upon short circuit current. When, however, the sera were injected into the frog ventricle so that they reached the skin by way of the blood vessels, a significant decrease in initial short circuit current was observed. No decrease was observed when the contralateral half of the abdominal skin was perfused in the same fashion with normal rabbit serum. These results suggest an effect upon cell permeability or sodium transport by the intact whole tissue when CA reaches it by way of the blood vessels, but not when it is simply juxtaposed to the outside or inside of the tissue.

Intracellular Ions and Biologic Functions in Kwashiorkor. Jack MetcofF* and Silvestre Frenk, Chicago, III. and Mexico, D.F., Mexico.

Previous muscle biopsy studies on 30 malnourished Mexican children indicated that death from chronic severe infantile malnutrition (kwashiorkor) was associated with intracellular accumulation of water and $\mathrm{Na}$, plus reduced intracellular concentrations of $\mathrm{K}, \mathrm{Mg}$, and organic P. Recovery was associated with reversal of these phenomena. A few biopsies suggested that muscle pyruvate and $\alpha$-ketoglutarate decreased, and lactate increased, as death occurred. It was hypothesized that activity of some ion-dependent enzyme system might be impaired, contributing to the fatal accumulation of intracellular water and $\mathrm{Na}$. To assess this hypothesis, muscle biopsies were obtained on 15 additional malnourished children. The characteristic cellular electrolyte lesions were verified. In eight children, including three of five who subsequently died, muscle contents of the intermediates-high-energy bond phosphoenolpyruvate ( PEP), pyruvate, $\alpha$-ketoglutarate, oxalacetate, and isocitrateas well as activities of the enzymes, pyruvic kinase (PK) (catalyzing $\sim \mathrm{P}+\mathrm{ADP} \rightarrow \mathrm{ATP} \sim \mathrm{P}$ ), lactic, isocitric, and malic dehydrogenases, glutamic-pyruvic and -oxalacetic transaminases, were measured and compared to those of six control subjects. Reductions of $\sim$ PEP, pyruvate, and $\alpha$-ketoglutarate were found in repeat biopsies shortly before or at death from severe malnutrition. The enzyme activities (mmoles/min/100 $\mathrm{g}$ dry fatfree solids) often were increased, but rarely decreased. 
Apparently "normal" homogenate $\mathrm{PK}$ activity from a typical malnourished child who subsequently died then was studied at substrate $[\sim \mathrm{PEP}]$ ranging from 33 to $1,000 \mu$ moles per $\mathrm{L}$ and at $a$ ) [K] $147,[\mathrm{Na}] 16.8$ equivalent to normal controls; b) [K] $147,[\mathrm{Na}] 50.2$; and $c$ ) [K] $63,[\mathrm{Na}] 50.2 \mathrm{mM} / \mathrm{L}$, characterizing fatal kwashiorkor. Activities were inversely proportional to $[\sim \mathrm{PEP}]$ and reaction velocities $(\mathrm{Km})$ for the unpurified muscle enzyme in the above systems were $a$ ) $6.5, b$ ) $7.5, c$ ) $8.0 \times 10^{-5} \mathrm{M}$. Since activity was virtually optimal in the control system, it seems likely that cellular $\sim \mathrm{PEP}$ depletion and distorted ion concentrations inhibit $\mathrm{PK}$ function (or vice versa) contributing to death in kwashiorkor.

Distribution and Properties of Anaphylactic and Venominduced, Slow-reacting Substance in Guinea Pigs. E. Middleton, Jr. ANd G. B. Phillips,* New York, N. Y.

Slow-reacting substance (SRS), which produces delayed contraction ( 5 to 30 seconds) of guinea pig ileum, as well as of human bronchial muscle, in the presence of atropine and antihistamines, is liberated from anaphylactically sensitized lung of several animal species, including man, on reaction with antigen. Perfusion of guinea pig lung with snake venom also produces SRS activity. Sensitized and normal guinea pig tissues were incubated with antigen or snake venom (Naja naja), and the supernatants assayed on guinea pig ileum for SRS directly and after extraction or dialysis procedures. Tissue lipid extracts were examined for SRS activity before and after venom treatment. 1) Sensitized lung incubated with antigen or venom liberated SRS (also normal lung with venom); much less activity arose from aorta on similar treatment and minimal, if any, activity from heart, liver, spleen, ileum, uterus, trachea, brain, and diaphragm of sensitized guinea pigs. 2) Normal lung lipid extracts displayed SRS activity, with a latent period before contraction of the ileum of about 1 to 3 minutes. Lipid extracts of kidney and liver showed no SRS activity; heart and brain lipid extracts, however, showed typical SRS activity. 3) Normal lung lipid extracts exposed to venom produced typical SRS activity. 4) Dialysis experiments showed that a) SRS in Tyrode's supernatants from lung-antigen reactions was poorly dialyzable; however, when these supernatants were lyophilized, extracted with $80 \%$ ethanol, dried, and re-extracted with Tyrode's, the SRS was readily dialyzable; $b$ ) the atypical SRS of lung lipid extracts was not dialyzable; and c) SRS present in lung lipid-venom mixtures was poorly dialyzable. 5) Anaphylactic SRS after lyophilization and ethanol extraction, and venom-induced SRS were extractable by ether from acid solution. Antigen-antibody reaction and venom are strikingly similar in their capacity to produce SRS, which appears to be an acidic, ether-soluble, small molecule.
Depression of Glucose Oxidation by Palmitate and by Acetoacetate in the Perfused Rat Heart. P. R. MiNton AND M. S. Raben,* Boston, Mass.

The influence of palmitate and of acetoacetate on the oxidation of uniformly labeled glucose- $\mathrm{C}^{14}$ to $\mathrm{C}^{14} \mathrm{O}_{2}$ was studied on contracting but nonworking hearts from fed rats during a 45-minute retroaortic coronary perfusion with recycled modified Krebs-Ringer bicarbonate solution, containing $2 \%$ albumin and $100 \mathrm{mg}$ per $100 \mathrm{ml}$ glucose. Production of $\mathrm{C}^{14} \mathrm{O}_{2}$ was least during the first 15 minutes ( $15 \%$ of total produced in 45 minutes), although loss of radioactivity from the medium was greatest in this period. Calculated from $\mathrm{C}^{14} \mathrm{O}_{2}$ recovered in the 45-minute period, the glucose oxidized was 2.6 $\mathrm{mg}$ per $\mathrm{g}$ wet weight per hour. Glucose oxidation was reduced by $82 \%$ when the medium contained palmitate $(1 \mathrm{mEq} / \mathrm{L})$ and by $75 \%$ when it contained acetoacetate $(4 \mathrm{mEq} / \mathrm{L})$. Palmitate and acetoacetate inhibited oxidation of glucose without decreasing its uptake as calculated from the disappearance of chemically determined glucose. Of the $\mathrm{C}^{14}$ lost from the medium, $\mathrm{C}^{14} \mathrm{O}_{2}$ accounted for $32 \%$ with glucose alone, $10 \%$ with palmitate present, and $9 \%$ with acetoacetate. By contrast, with acetoacetate as sole substrate $(4 \mathrm{mEq} / \mathrm{L}), \mathrm{C}^{14} \mathrm{O}_{2}$ from acetoacetate-3- $\mathrm{C}^{14}$ accounted for $79 \%$ of the $\mathrm{C}^{14}$ lost from the medium, representing use of $5.4 \mathrm{mg}$ per $\mathrm{g}$ per hour; and of the total $\mathrm{C}^{14} \mathrm{O}_{2}$ produced, $34 \%$ appeared in the first 15 minutes. Glucose had little effect on the oxidation of acetoacetate or of palmitate in the concentrations used. Both long chain fatty acid and ketoacid were preferred to glucose as substrate for oxidative metabolism in these experiments. The observed pattern may explain several phenomena occurring at times of increased availability of the preferred substrates, including the increase of cardiac glycogen content with fasting, and the cardiac "glycostatic" effect of growth hormone.

The Determination of Sodium in Body Fluids by Means of the Glass Electrode. EDWARD W. MOORE AND Donald W. Wilson, Boston, Mass. (introduced by Thomas C. Chalmers).

Until the present time, there has been no method for the direct determination of activity of alkali ions in biological fluids, physiologic data having been expressed solely in terms of concentration. The following data summarize the application of the mathematical and electrochemical techniques for the determination of $\mathrm{pH}$ to the potentiometric measurement of sodium ion in body fluids. Sodium activity $\left(\alpha_{\mathbf{N a}^{+}}\right)$was determined in a total of 312 samples of urine, serum CSF, whole blood, and plasma from normal subjects and hospitalized patients, using a highly selective capillary electrode composed of $\mathrm{Na}_{2} \mathrm{O}-\mathrm{Al}_{2} \mathrm{O}_{3}-\mathrm{SiO}_{2}$ glass (Eisenman). Analyses required about 3 minutes in $0.5-\mathrm{ml}$ undiluted samples, and duplicate variability was less than that for the flame photometer ( $\mathrm{SD} 0.68$ vs $1.17 \mathrm{mEq} / \mathrm{L}$ in urine, respectively). In all fluids studied, $\alpha_{\mathrm{Na}^{+}}$was linearly related to photometric concentration $\left(\mathrm{C}_{\mathrm{Na}_{\mathrm{P}}}\right)$. In order to ex- 
press electrode activities in terms of concentrations $\left(\mathrm{C}_{\mathrm{Na}_{\mathrm{E}}}\right)$, it was necessary to derive appropriate empirical mean activity coefficients $\left(\gamma_{\mathrm{Na}^{+}}\right): \mathrm{C}_{\mathrm{Na}_{\mathrm{E}}}=\alpha_{\mathrm{Na}^{+}} / \gamma_{\mathrm{Na}^{+}}$. This has been accomplished for each fluid. The average deviation of $\mathrm{C}_{\mathrm{Na}_{\mathrm{E}}}$ from $\mathrm{C}_{\mathrm{Na}_{\mathrm{P}}}$, without regard to sign, was $2.6 \mathrm{mEq}$ per $\mathrm{L}$ in urine, $2.2 \mathrm{mEq}$ per $\mathrm{L}$ in $\mathrm{CSF}$, and $1.3 \mathrm{mEq}$ per $\mathrm{L}$ in serum, whole blood, and plasma. In urine $\gamma_{\mathrm{Na}^{+}}$(ordinate) vs $\alpha_{\mathrm{Na}^{+}}$was approximated by the hyperbola $Y=0.66+(4.04 / X)$. Suppression of urine $\alpha_{\mathrm{Na}}+$ below that expected for aqueous $\mathrm{NaCl}$ solutions was found to be related to potassium concentration, since $\mathrm{C}_{\mathrm{Na}_{\mathrm{E}}}$ was closely approximated by $\alpha_{\mathrm{Na}^{+}} / \gamma_{\mathrm{Na}^{+} \mathrm{K}}$. Glucose $\left(2\right.$ to $6 \%$ ) increased urine $\alpha_{\mathrm{Na}^{+}}$slightly, while urea, uric acid, and protein had no effect. Erythrocytes and serum proteins had no adverse effects on electrode potential. The activity and concentration of sodium ion have been determined rapidly and accurately with the glass electrode, and similar studies with other glasses indicate the feasibility of quantitating $\mathrm{K}^{+}, \mathrm{NH}_{4}^{+}, \mathrm{Ca}^{++}$, and $\mathrm{Mg}^{++}$in biologic fluids.

Splenectomy and Survival of Macrocytes Resulting from Erythropoietic Stimulation. R. R. Moores, F. STOHLMAN, JR.* AND G. BRECher, Bethesda, Md.

Initially, macrocytic red cells are produced by rats in response to intense erythropoietic stimulation, e.g., phenylhydrazine-induced anemia, blood loss, exposure to simulated altitude, or administration of erythropoietin. These macrocytes have a markedly shortened life span. Studies to date point to a dose-response relationship between the intensity of stimulation and the degree of response. Also, the initial cohorts of cells are more macrocytic and have a shorter life span than ensuing ones. The possible role of the spleen in removal of these large cells was evaluated by bleeding or giving phenylhydrazine to pathogen-free, female Sprague-Dawley rats approximately 2 weeks after splenectomy. Red cell survival was measured with radioiron $\left(\mathrm{Fe}^{69}\right)$ and from cell size distribution curves obtained with a model B Coulter counter. The phenylhydrazine-damaged cells were totally removed from the circulation, although at a slower rate in the splenectomized than in the intact animals. However, neither the degree of macrocytosis nor the life span of the macrocytic cells was affected by splenectomy in either the bled or phenylhydrazine-treated animals. These observations suggest that shortened survival of red cells produced in response to intense erythropoietic stimulation is not due merely to mechanical factors related to the macrocytosis.

The Nature of Pulmonary Capillary Blood Flow and Gas Exchange. Eugene Morkin, O. Robert Levine, Frederick O. Bowman and Alfred P. Fishman,* New York, N. Y.

Rhythmic fluctuations in intrapulmonary pressure, synchronous with the heart beat, have been recorded plethysmographically in man. However, these observa- tions have not settled whether alveolar-capillary gas exchange, as well as the pulmonary capillary blood flow, is continuous or interrupted and pulsatile. In the present study, closed-chest dogs were used to test the effects of arresting unilateral pulmonary blood flow and gas exchange on the contour of the ipsilateral intrapulmonary pressures. The experiment involved: 1) surgical exteriorization of the left bronchus for the ventilation of one lung while the other was being tested, 2) placement of a balloon-tipped catheter so that blood flow through the test lung could be interrupted at will, 3) introduction of cardiac and bronchial catheters so that intrapulmonary pressure pulses could be related to cardiodynamic events, and 4) pneumographic monitoring of changes in thoracic circumference. The intrapulmonary oscillations were recorded during inflation of the test lung with air and with $80 \%$ nitrous oxide in oxygen; similar recordings were made when the blood flow through the test lung was interrupted. Separate spirometric and manometric measurements were used to verify that blood flow to the test lung had been arrested. It was found that: 1) the intrapulmonary pulsations on air and nitrous oxide differed principally with respect to the slope of their baselines, rather than to their contour; 2) the uptake of nitrous oxide fluctuated irregularly and continued without pause throughout the cardiac cycle; and 3) arrest of blood flow to the test lung abolished the slope of the nitrous oxide uptake so that the nitrous oxide and air oscillations became superimposable. The results indicate that, under the conditions of these experiments, alveolar-capillary gas exchange and pulmonary capillary blood flow are continuous processes and undergo only slight deviations from their mean values during each cardiac cycle.

Studies on the Relationship of Angiotensin to Hypertension of Renal Origin. RusSELl E. MORRIs, JR., Patricia A. Ransom and John Eager Howard, Baltimore, Md. (introduced by Samuel P. Asper, Jr.).

The decreased flow of blood to a kidney with the production of ischemia can cause hypertension. It has been postulated that this hypertension is due to a circulating humoral agent, angiotensin, produced by the action of the kidney enzyme, renin, on plasma globulins. Whether angiotensin is produced in all types of kidney disease or only by an ischemic kidney is unknown. Forty patients have been evaluated in this study to correlate the relation of angiotensin to hypertension. The diagnostic studies used were renal arteriogram, split renal function tests, renal scintiscan, and peripheral arterial and renal venous blood determinations of angiotensin. Angiotensin was isolated by a method of chemical extraction developed in this laboratory and determined by bioassay in the rat. This procedure is sensitive to $0.01 \mu \mathrm{g}$ angiotensin in $50 \mathrm{ml}$ of blood. Twenty patients were diagnosed as having essential hypertension and in these angiotensin was not found. Angiotensin was not found in one patient with pheochromocytoma, one with primary aldosteronism, and in five patients with malig- 
nant hypertension. After renal surgery, three of these patients with malignant hypertension showed no change in blood pressure. Thirteen patients were diagnosed as having renal hypertension with a demonstrated unilateral or bilateral renal lesion. In these, angiotensin was found both in peripheral arterial blood and in renal venous blood from the ischemic kidney; angiotensin was not found in the renal venous blood from the uninvolved kidney or in the peripheral venous blood. Six of these patients received unilateral nephrectomy and are improved; angiotensin was not found in the peripheral arterial blood postoperatively. From these data, it is believed that concentrations of angiotensin sufficient to cause hypertension by their direct effect on blood vessels are present only in renal ischemia. Angiotensin has not been demonstrated in essential or malignant hypertension.

The Effect of Endotoxin on Cardiac Output and Hepatic Flow in Normal and Tolerant Rabbits. JоHN M. Moses and William J. MacIntyre, Cleveland, Ohio (introduced by Robert $\mathrm{H}$. Ebert).

Endotoxins are known to alter the phagocytic function of the reticuloendothelial system (RES). After large doses of intravenously administered endotoxins, RES activity is initially reduced. Several factors influencing RES activity have been implicated after endotoxin administration. One of these factors, organ perfusion, has not been emphasized in spite of the knowledge that endotoxins induce profound systemic changes in circulation. The fact that certain toxic substances produce marked changes in hepatic circulation and the observation that rabbits resistant to the lethal effects of endotoxin may have impaired hepatic blood flow, suggested the possibility that local organ perfusion might be altered in the absence of more widespread failure of circulation after endotoxin. Both normal rabbits and those rendered tolerant to endotoxin by a series of daily injections of bacterial lipopolysaccharide were studied before and after the injection of large doses of lipopolysaccharide. A simple method was devised to enable simultaneous and repeated determinations of both cardiac output and hepatic blood flow by means of single injections of carrier-free colloidal radiogold. An arteriovenous shunt was established between the carotid artery and contralateral jugular vein. The diverted arterial blood was led through a scintillation counter by means of a polyethylene tube so that radioactivity in the blood could be monitored continuously. After the injection of gold, radioactivity in blood ejected from the heart inscribed a curve which permitted calculation of cardiac output. The subsequent disappearance of radioactivity established a measure of hepatic blood flow. The results showed that reduced hepatic blood flow was associated with a fall in cardiac output in both normal and tolerant rabbits. Impaired circulation was short-lived in tolerant rabbits but progressive in normals. The data suggest that alterations in organ perfusion may be of primary importance in influencing RES activity after the injection of endotoxin.
Aryl Amine Acetylation in Human Red Cells. Arno G. Motulsky* and Laura Steinmann, Seattle, Wash.

Biotransformation of isoniazid (INH) varies considerably between individuals. Blood levels in family members after administration of $\mathrm{INH}$ indicate that a single gene controls the major pathway of drug metabolism. Three classes of individuals can be defined: homozygotes with the double dose of the gene, heterozygotes, and homozygotes lacking this gene. Pharmacologic evidence suggests that this pathway concerns hepatic acetylation of INH. It is uncertain whether para-aminosalicylic acid (PAS) is acetylated by the same mechanism. Since INH blood levels cannot characterize the enzymatic mechanism underlying these genotypic differences, red cells were selected as a potentially useful model for the direct study of acetylation. Acetylation of PAS and of paraaminobenzoic acid (PABA) could be demonstrated in hemolysates using an acetate-CoA-ATP system to produce acetyl-CoA as acetyl donor. When citrate was substituted for acetate, even stronger acetylation occurred. These reactions indicate the presence of acetateactivating enzyme, citrate-splitting enzyme, and aryl amine acetylase in red cells. Levels of acetylating activity were high in reticulocytes but remained significant even in mature cells. When isoniazid and sulfanilamide were substituted as acetyl acceptors, no acetylation was found. INH and sulfanilamide also failed to inhibit acetylation of PABA, while PAS, aminopterin, and amethopterin strongly inhibited PABA acetylation by competing for available acetyl-CoA. Population studies of PAS and PABA acetylation in both Caucasian and Negro subjects indicated that variability followed a normal distribution as contrasted with the multimodal curve seen with INH inactivation. These data demonstrate the presence of an aryl amine acetylase in human red cells which reacts with PAS and related compounds but not with INH. This red cell acetylase is controlled by a genetic mechanism different from that regulating INH inactivation. These findings strengthen the suggestion that hepatic acetylation of INH and PAS is carried out by different acetylating enzymes.

Antihypertensive Principle from Renal Medulla. E. E. Muirhead,* J. W. Hinman, E. G. Daniels, M. KoSInski ANd B. Brooks, Detroit and Kalamazoo, Mich.

Hypertensive cardiovascular disease occurs in the dog after renal ablation plus sodium and dietary protein. This form of renoprival hypertension is prevented by explanted whole kidney or renal medulla, a saline extract of renal medulla given intravenously, and a medullorenal extract refined by $55 \%$ ethanol extraction. Prevention of canine renoprival hypertension affords an assay procedure for purifying the medullorenal antihypertensive principle. Direct $55 \%$ ethanol extraction of canine and porcine renal medulla yielded a product preventive against canine renoprival hypertension. Subsequent preparations were derived from porcine kidneys and tested in dogs. Removal of ethanol after 55\% ethanol extraction, followed by Sephadex chromatography, permitted use of 
kidneys after partial cortical dissection. Elution of the column yielded two fractions. The component active against hypertension appeared to be of low molecular weight $(<2,000 \mathrm{~mol} \mathrm{wt})$. The active principle was not retained on Dowex-50 $\left(\mathrm{H}^{+}\right)$resin and presumably was not an amino acid or peptide. The $55 \%$ ethanol extract was extracted first with Skellysolve B and then with ethyl acetate. The solids from ethyl acetate dissolved in ethanol and given by mouth $(1 \mathrm{mg} / \mathrm{kg} / \mathrm{day})$ protected against renoprival hypertension. The active material was slightly water-soluble and did not contain free amino groups, phenolic hydroxyl groups, or reducing carbohydrates. Further solvent fractionation yielded a preparation active in doses of $50 \mu \mathrm{g}$ per $\mathrm{kg}$ per day. Protection against hypertension also altered significantly other parameters of nondialyzed dogs. The average change from control for 13 protected nonhypertensive dogs and for 5 hypertensive animals was: for blood urea nitrogen, +188 and $+316 \mathrm{mg}$ per $100 \mathrm{ml}$; for serum potassium, +1.67 and $+2.96 \mathrm{mEq}$ per $\mathrm{L}$; for reduced glutathione of erythrocytes, -2.6 and $-24.5 \mathrm{mg}$ per $100 \mathrm{ml}$ red blood cells. Cardiovascular injury was not observed when hypertension was prevented. A principle from porcine renal medulla protected against canine renoprival hypertension when given orally in relatively small amounts.

Incorporation of 17-Hydroxy-Pregnenolone- $H^{3}$ into Cortisol by the Human Adrenal In Vitro and by the Dog Adrenal In Vivo. Patrick J. Mulrow, George L. Cohn, Albert A. Kuljian and William F. Ganong, New Haven, Conn. and San Francisco, Calif. (introduced by Philip K. Bondy).

Studies of adrenal steroid biosynthesis indicate that pregnenolone is incorporated into cortisol via pregnenolone $\rightarrow$ progesterone $\rightarrow$ 17-hydroxy-progesterone $\rightarrow$ 11-deoxycortisol $\rightarrow$ cortisol. However, 17-hydroxylation of pregnenolone is considered the first step in adrenal androgen biosynthesis. The present study demonstrates that a major pathway of cortisol biosynthesis is by way of 17-hydroxy-pregnenolone in normal and hyperplastic human adrenals. Slices of four "normal" adrenals obtained by surgery from two patients with prostatic cancer were incubated with 17-hydroxy-pregnelone- $\mathrm{H}^{3}$. Eight to $19 \%$ of the radioactivity was incorporated into the cortisol isolated from the incubation media. Intermediates, 17-hydroxy-progesterone- $\mathrm{H}^{3}$ and 11-deoxycortisol- $\mathrm{H}^{3}$, were also isolated. Little or no corticosterone- $\mathrm{H}^{3}$ was detected. Radiochemical purity of the free alcohols and their derivatives was achieved by paper chromatography. Slices of a hyperplastic adrenal gland from a patient with Cushing's syndrome were incubated with equimolar amounts of 17-hydroxy-pregnenolone- $\mathrm{H}^{3}$ and progesterone- $\mathrm{C}^{14} ; 6 \%$ of 17-hydroxy-pregnenolone and $9 \%$ of progesterone- $\mathrm{C}^{14}$ were incorporated into cortisol. 11-Deoxycortisol- $\mathrm{H}^{3}$ was also detected. Studies using progesterone- $\mathrm{H}^{3}$ as the substrate in normal and in three hyperplastic adrenal glands demonstrated 7 to $16 \%$ incorporation of progesterone- $\mathrm{H}^{3}$ into cortisol. In three dogs who were receiving a constant infusion of $\mathrm{ACTH}$, equimolar amounts of 17-hydroxy-pregnenolone- $\mathrm{H}^{3}$ and progesterone- $\mathrm{C}^{\mathbf{1 4}}$ were infused directly into the isolated adrenal gland in vivo. Cortisol containing $\mathrm{H}^{3}$ and $\mathrm{C}^{14}$ was isolated from adrenal vein blood. The relative amounts of the two precursors incorporated into cortisol, expressed as the ratios of per cent incorporation of 17 -hydroxy-pregnenolone- $\mathrm{H}^{3} /$ progesterone- $\mathrm{C}^{\mathbf{1 4}}$, were 0.65 , 0.76 , and 1.8. In conclusion, a major pathway for cortisol biosynthesis, bypassing progesterone, has been demonstrated to exist in the human adrenal in vitro and in the dog adrenal in vivo. A pathway via 17-hydroxy-pregnenolone $\rightarrow 17$-hydroxy-progesterone $\rightarrow 11$-deoxycortisol $\rightarrow$ cortisol has been shown in vitro.

The Fate of the Platelet. J. F. Mustard, E. A. MurPhy And G. A. Robinson, Toronto and Guelph, Ontario, and Baltimore, Md. (introduced by R. F. Farquharson).

Calculations of platelet life span from data obtained in radioactive labeling experiments have varied widely because the mode of destruction has hitherto been obscure. Estimates based on the assumption of random destruction have been about 3 to 4 days; the same data yield an estimate of 10 to 12 days if death is assumed to be due to platelet aging. It is clearly important to decide which of these mathematical models is the more appropriate, but attempts to do so by scrutiny of survival curves are frustrated by even small experimental errors. We have therefore had recourse to indirect methods. 1) If destruction is due to senescence, then the shape of the survival curve should vary according to the age of the platelets labeled. If, however, destruction is random, it should be independent of platelet age. In swine and in dogs, platelets were simultaneously labeled in vivo with $\mathrm{S}^{35}$ (which is incorporated in developing platelets only) and diisopropyl fluorophosphate (DFP) ${ }^{32}$ (which adheres indiscriminately to circulating platelets of all ages). The plots of platelet survival curves on semilogarithmic graph paper yielded two parallel straight lines, showing that destruction occurred at the same rate in the two groups of platelets. This suggests random destruction. 2) By the $\mathrm{DFP}^{32}$ method, we found in man that platelet survival computed on either mathematical model was prolonged by high doses of bishydroxycoumarin in 12 subjects $(p<0.001)$, by $8,000 \mathrm{U}$ of heparin t.d.s. in 14 $(p<0.01)$, by restriction or change of dietary fat in 7 $(p<0.01)$, and by restriction of smoking in $7(p<0.01)$. It seems most plausible to suppose that these varied effects are mediated through the environment of the platelet. It is concluded that the fate of the platelet is determined mainly by its environment rather than by its intrinsic metabolism, and that its survival is best computed as an exponential half-life.

Digital Hemodynamics in Hypertension with Particular Emphasis on Venomotor Tone. Rudolph J. NAPOdano, Francis S. Caliva, Robert M. Stafford and Richard H. Lyons,* Syracuse, N. Y.

There is ample evidence that the increased peripheral resistance in hypertension results from narrowing of 
small arteries and arterioles. There is little information concerning the state of the venous circulation. Since the small veins and venules possess smooth muscle elements which are anatomically and physiologically similar, an investigation was undertaken to assay their role in the over-all increase in resistance. Ten normotensive and 15 hypertensive subjects were studied three or more times by the technic of digital rheoplethysmography. The patients were divided into groups according to age, and the parameters investigated were digital blood flow, digital arterial and venous pressures, vascular resistance, and tone in the small veins. Calculated peripheral resistances in all the hypertensives were significantly higher than in the normotensives. In hypertensive patients under 50 years of age, there was a significantly lower mean blood flow and higher venous pressure than in normals of the same age group. Such differences were not evident in the patients over 50 years. Analysis of the rate of inflow curves for the first two pulse cycles revealed a moderate degree of increased venomotor activity in the hypertensives. Criteria were designed for grading the status of existing tone from 0 to IV. Of the normotensives less than 50 years old, three of six showed no evidence of increased venomotor tone, while the other three were classed as grades I or II. None of these normotensives demonstrated grade III or IV venomotor tone. Of the seven hypertensives under 50 , five demonstrated grade III or IV venomotor activity. In the age group over 50, three of four normotensives and all eight hypertensives demonstrated grade I to III venomotor tone. It thus appears likely that the increased peripheral resistance in hypertension, in part, includes the small veins. Experimentally, this is most evident in the subjects under 50 years of age.

The Relationship of Increased Erythropoiesis to Globin Synthesis in Man. David G. Nathan and Frank H. GARDNER,* Boston, Mass.

This study was designed to compare the effects on heme and globin synthesis of androgen-induced polycythemia with other states of increased erythropoiesis. Glycine-2-C $\mathrm{C}^{14}$ was administered i.v. to three individuals before and after sufficient androgen therapy to increase the total red cell volume (patient group A). Daily determinations of $\mathrm{C}^{14}$-heme and -globin specific activity (SA) of peripheral blood were performed for 1 week. Similar studies were done in a patient who was polycythemic due to a virilizing adrenal tumor, a patient with polycythemia vera, and a patient with iron deficiency (patient group B). These patients did not receive androgens. Hypochromia was a characteristic of the peripheral blood after androgen treatment (group A) and in group $\mathrm{B}$. The curves of $\mathrm{C}^{14}$-heme and -globin SA in group A were compared both to similar data in normal individuals and to the pretreatment curves. The data of group B were compared to those of normal individuals. The results were very similar in groups $A$ and $B$. The heme curves differed slightly from pretreatment on normal values in that the peak increment of SA occurred
2 to 3 days rather than 3 to 4 days after glycine administration. The globin curves were markedly altered. They assumed a hyperbolic rather than a sigmoid shape, so that the peak increment of $\mathrm{C}^{14}$-globin SA occurred 1 or 2 days rather than 5 days after glycine administration. These results indicate that the kinetics of heme and globin synthesis due to androgen stimulation do not differ from such kinetics in other states of increased erythropoiesis. Globin synthesis or "turnover" is enhanced out of proportion to heme synthesis or "turnover" when erythropoiesis is stimulated. This enhancement is apparently most marked during the later stages of erythroid cell maturation.

Differential "Aging" in Two Types of Bone. GEORGE Nichols, JR.,* Boston, Mass.

Methods have now been developed in this laboratory for measuring in vitro the metabolic activity of bone cells, and for tracing incorporation of substrates into metabolic end products and components of bone organic matrix. Thus, studies are possible of the cellular metabolic basis of the differential rates of growth and remodeling which occur in different skeletal areas with increasing age. Answers have been sought to three questions: What changes occur in cell metabolic patterns and rates with age? Are these different in trabecular and cortical bone? Are these the result of changes in the number of cells, their metabolism, or both? Cell content, together with rates of incorporation of label from glucose, glycine, and proline into metabolic end products, cells, and collagen, have been measured in cortical and trabecular bone from metaphyses of rats ranging in age from 3 weeks to 8 months. Cell content, estimated by DNA and total N, is lower in cortical than in trabecular bone and decreases with age, while in trabecular bone cellularity changes little. Glucose consumption, calculated per $100 \mathrm{mg}$ fresh bone, is $25 \%$ lower in cortex at 3 weeks and decreases steadily with age, while in trabecular bone no decrease was observed. The percentage of glucose label incorporated into various end products, cells, and collagen was the same in both bone types at all ages. Calculation on a cell $\mathrm{N}$ basis revealed that changes in cortex were the result of changes in cell content, not in cell activity. Studies of amino acids, however, showed progressive decreases in incorporation into both cells and collagen with age when calculated on either basis. In contrast to glucose, proline incorporation was 30 to $50 \%$ higher in cortical than in trabecular bone. Thus, patterns of glucose and amino acid metabolism change with age in different ways and for different reasons depending on the bone type.

Evidence for Abnormal Lipoprotein Synthesis in Two Varieties of Idiopathic Hyperlipemia. William H. R. Nye and Christine Waterhouse,* Rochester, N. Y.

In order to explore the differences between fat-induced and carbohydrate-induced idiopathic hyperlipemia, a typical patient of each type was studied on a metabolic ward. The first patient has been observed at intervals during 
the past 17 months. Before diet was begun, plasma was markedly hyperlipemic, and individual phosphatides were present in proportions that could not be explained by alterrations in ratios among normal lipoprotein classes. With a low fat, isocaloric diet, phosphatide proportions reverted to normal, and the hyperlipemia cleared. The second patient, who was obese, has had two periods of study during the past 10 months, and was under continuous dietary supervision for 4 months during the second period. Phosphatides were initially normal. On a low fat, high carbohydrate diet, hyperlipemia increased and phosphatides remained normal. On a low calorie, normal fat, low carbohydrate diet, hyperlipemia cleared, but proportions among individual phosphatides changed in directions not explainable by shifts in normal lipoprotein class ratios. After the patient had lost $\mathbf{4 0}$ pounds over a 3-month period, the low carbohydrate diet was made isocaloric. Plasma remained clear, and phosphatide proportions became normal again. Glucose tolerance, which was decreased in this patient initially, became normal after weight loss. Both patients showed a good response to heparin, and in both cortisone caused a decrease in total lipids without altering phosphatide proportions. We postulate that the first patient synthesized abnormal lipoproteins in response to exogenous fat, but was able to synthesize normal lipoproteins and maintain normal plasma lipid levels on a diet in which carbohydrate predominated. The second patient synthesized abnormal lipoproteins only when the major source of energy was from exogenous plus endogenous fat; however, the hyperlipemia itself was correlated with a high carbohydrate intake, suggesting that this aspect of the patient's abnormality was quantitative rather than qualitative in nature.

The Metabolism of Warfarin. ROBERT A. O'ReILLy, Paul M. Aggeler* and LoIs Leong, San Francisco, Calif.

Variable responses to coumarin anticoagulants have not been explained by detailed clotting factor analysis. These variations are conditioned by differences in drug metabolism. While warfarin is widely used in anticoagulant therapy, its metabolic behavior in man has not been previously studied. Since no applicable method was available, we developed an assay based on ultraviolet absorption spectrophotometry. Thirteen normal subjects were given single oral doses of warfarin, $1.5 \mathrm{mg}$ per $\mathrm{kg}$; 6 received the same dose i.v. The following results were obtained (mean $\pm \mathrm{SD})$ : biologic half-life $\left(\mathrm{t}_{1}\right), 44 \pm 13$ hours; elimination rate, $1.6 \pm 0.5 \%$ per hour; apparent volume of distribution (Vd), $12 \pm 1 \%$ body weight; time of maximum plasma levels, $4.6 \pm 2.0$ hours. A significant correlation $(p<0.05)$ was found between $t_{i}$ and the maximum prothrombin time (Quick), and a highly significant correlation $(p<0.01)$ between the 48-hour warfarin level and 48-hour prothrombin time. Various parameters of warfarin and bishydroxycoumarin (Dicumarol) metabolism were compared. Gastrointestinal $a b$ sorption: On the basis of plasma drug levels, biologic effect, and drug recovery in the stool, warfarin is absorbed rapidly and completely, and Dicumarol slowly and incompletely. Absorption rates calculated from the plasma data indicate higher maximum rates for Dicumarol that become slower before the dose is completely absorbed. Elimination: The $t_{3}$ for oral and i.v. warfarin and for high oral Dicumarol doses is independent of dose size; for all i.v. and low oral Dicumarol doses, $t_{1}$ varies directly with dose size. Excretion of warfarin in urine and stool is essentially 0 , and of Dicumarol is 0 in urine and $20 \%$ in stool. About 0.25 of a warfarin dose is recovered from urine as a metabolite. Plasma protein binding of warfarin is $97 \%$, and of Dicumarol $99 \%$; these values may be related to the low Vd. In normal subjects the prothrombinopenic effects of the coumarin anticoagulants correlate well with their metabolic fate.

\section{A Serum Factor that Agglutinates Stored Erythrocytes. Faruk L. Ozer, David Miller and Hugh Chaplin, JR.,* St. Louis, Mo.}

The authors have encountered a hyperglobulinemic human serum which causes strong agglutination of stored erythrocytes while fresh erythrocytes remain unaffected. Agglutinating activity was demonstrable in serum diluted $1: 100,000$ and withstood heating at $60^{\circ} \mathrm{C}$ for 10 hours. Activity was associated with a paraprotein fraction of gamma mobility and a sedimentation constant of 19S. Protein precipitable at low ionic strength possessed strong agglutinating activity when redissolved, with loss of activity after treatment with penicillamine. Agglutinating activity was also inhibited by exposure to such sulfhydryl-binding and disulfide-breaking agents as $p$-hydroxy-mercuribenzoate, Na arsenite, GSSG, GSH, and cysteine. Activity was diminished by absorption with aged erythrocytes and erythrocyte ghosts, and could be eluted from the washed cell membranes by heating at $56^{\circ}$ C. However, absorption of the serum factor was completely blocked by pretreatment of ghosts with $p$-hydroxymercuribenzoate. The alterations which rendered erythrocytes agglutinable by the serum factor progressed rapidly at $37^{\circ} \mathrm{C}$, slowly at $4^{\circ}$, and could be prevented or diminished by addition of acid citrate dextrose or adenosine to the suspending medium. Agglutinability was hastened by exposure to methylene blue (preventable by adenosine in normal cells but not in glucose-6-phosphate dehydrogenase-deficient cells) and also by inhibitors of glycolysis (2-deoxyglucose, $\mathrm{Na}$ arsenite, $\mathrm{Na}$ arsenate, $\mathrm{Na}$ fluoride, iodoacetate) and by $p$-hydroxy-mercuribenzoate. A close relationship of agglutinability to nonviability was supported by in vivo studies demonstrating markedly increased survival of the nonagglutinable cells in banked blood stored for 33 days. The present studies indicate that 1) the serum factor is a macroglobulin; 2) the agglutination reaction is inhibited by exposure of the macroglobulin and the erythrocyte membrane to reagents which affect sulfhydryl and disulfide groups; 3) agglutinability results from impaired erythrocyte metabolism; and 4) separation of nonviable cells in stored blood may become possible. 
The Metabolism of d-Aldosterone: Isolation and Chemical Identification of Metabolites; Distribution of Free Aldosterone and Metabolites in Tissues and Fluids under Physiologic Conditions. MaUrice M. PeChet,* Robert H. Hesse and Heinz Kohler, Boston, Mass.

From the incubation of the natural $d$-aldosterone with a homogenate of rat liver and a TPNH-generating system, the following metabolites were isolated and identified by precise chemical methods: $5 \alpha$ - $(4,5)$-dihydroaldosterone; $3 \beta$-hydroxy-5 $\alpha-(4,5)$-tetrahydroaldosterone; $3 \alpha$ hydroxy-5 $\alpha$ - $(4,5)$-tetrahydroaldosterone; and $3 \alpha$-hydroxy-5 $\beta-(4,5)$-tetrahydroaldosterone. Under these conditions $d$-aldosterone is reduced primarily to metabolites with $\mathrm{A} / \mathrm{B}$ trans $(5 \alpha)$ configuration. Similar results have been obtained with the isolated perfused rat liver. The hemiacetal structure characteristic of aldosterone remains intact. Experiments with tritiated aldosterone and radioactive-trapping experiments with synthetically prepared dihydroaldosterone indicate that the sequential steps in the metabolism of aldosterone are: $d$-aldosterone $\rightarrow$ dihydroaldosterone $\rightarrow$ tetrahydroaldosterone. In the intact rat, under basal conditions simulating the steady state, liver demonstrated the highest metabolic activity. No unchanged aldosterone but very high concentrations of conjugated metabolites are present in liver. The highest concentration of free aldosterone is found in the kidney, indicating a concentration of aldosterone by the kidney. The level of free aldosterone is low in serum, of the order of $10^{-9} \mathrm{M}$. The plasma aldosterone pool is in equilibrium with a larger pool contained in the total water space. Muscle tissue metabolizes $d$-aldosterone to the tetrahydro metabolites, and because of the large mass of muscle present this tissue may make a significant contribution to the metabolism of aldosterone. Experimental hemorrhage in normal and adrenalectomized rats receiving a constant aldosterone infusion of $0.3 \mu \mathrm{g}$ per hour is associated with an increase in free aldosterone in plasma, indicating that under these conditions metabolism of aldosterone is markedly diminished.

Sickling Phenomenon Produced by Hypertonic Solutions: A Possible Explanation for the Hyposthenuria of Sicklemia. Pasquale E. Perillie and Franklin H. Epstein,* New Haven, Conn.

The effect of hypertonic solutions upon sickling was examined in an attempt to clarify the inability of patients with sickle cell anemia to excrete a highly concentrated urine. Blood from such patients was mixed with solutions of $\mathrm{NaCl}$, urea, sucrose, or mannitol with osmolalites varying from 300 to $1,200 \mathrm{mOsm}$ per $\mathrm{kg}$. All tests were done at the temperature and oxygen tension of room air; the $\mathrm{pH}$ of the final mixture varied from 7.4 to 7.5. Sickling occurred in all solutions of $\mathrm{NaCl}$ with a final osmolality greater than $600 \mathrm{mOsm}$ per $\mathrm{kg}$ and could be reversed by diluting to concentrations below this. Sickling also occurred when blood was added to concentrated solutions of sucrose and mannitol, but not when blood was added to solutions of urea alone.
Normal red blood cells did not sickle when placed in a hypertonic medium. The viscosity of whole blood from sickle cell patients increased progressively when the osmotic concentration of the plasma was increased by adding $\mathrm{NaCl}$. No change, however, was noted in the viscosity of sickle hemoglobin solutions when solute concentration was similarly raised. The sickling of cells containing S-hemoglobin is promoted when they are immersed in hyperosmotic solutions, probably as a result of cellular dehydration. It is suggested that when such cells enter the hypertonic milieu of the renal medulla sickling is promoted, thereby increasing blood viscosity, restricting blood flow to the medulla, restraining active reabsorption of sodium in this region, and limiting the production of a high concentration of sodium in the medullary interstitial fluid.

Abnormal Myoglobin Chromatography in Childhood Muscular Dystrophy. Gerald T. PERKoff,* Robert L. Hill and Frank H. Tyler,* Salt Lake City, Utah and Durham, N. C.

Normal human myoglobin fractionates into three major $\left(F_{1}, F_{2}\right.$, and $\left.F_{3}\right)$ and three minor components on diethylaminoethyl cellulose columns. $F_{8}$ is separated further into six components by a gradient elution technique. $F_{1}$ and $\mathrm{F}_{2}$ comprise 70 to $85 \%$ of normal myoglobin and are acid and alkaline metmyoglobin, respectively. Their electrophoretic mobilities, end groups, peptide patterns ("fingerprints"), amino acid compositions, and sedimentationdiffusion constants are identical. $\mathrm{F}_{\mathrm{z}}$ myoglobins comprise 15 to $30 \%$ of normal myoglobin and are alkaline metmyoglobins with more rapid electrophoretic mobility than $F_{1}$ and $F_{2}$. One of the $F_{3}$ components, $F_{3} D$, has been analyzed and has a peptide pattern different from that of $F_{1}$ and $F_{2}$. In the visible spectrum at $\mathrm{pH} 8.6, \mathrm{~F}_{3} \mathrm{D}$ shows a single peak at $540 \mathrm{~m} \mu$ as does fetal myoglobin. The spectral characteristics of whole $F_{3}$ and fetal myoglobin are similar when these preparations are converted to ferromyoglobin, oxymyoglobin, and cyanmetmyoglobin, respectively. Definitive chemical characterization of fetal and $\mathrm{F}_{3} \mathrm{D}$ myoglobin must be accomplished before any conclusions concerning this spectral similarity are justified. Myoglobin from skeletal muscle of two childhood dystrophy patients contained a preponderance of $F_{3}$ (50 and $100 \%$ of the total myoglobin, respectively). In one instance $F_{1}$ and $F_{2}$, although markedly reduced in amount, were present and showed normal structural characteristics. The spectrum of whole alkaline metmyoglobin of childhood dystrophy reflects the preponderance of $F_{8}$. This probably explains the reported spectral "abnormalities" in myoglobin from dystrophic patients. The spectral characteristics, electrophoretic mobilities, and chromatographic patterns of myoglobins from two patients each with facioscapulohumeral and myotonic dystrophy were normal. These studies indicate an abnormal distribution of myoglobins in one form of progressive muscular dystrophy. The preponderance of $F_{3}$ in childhood dystrophy myoglobin may in part represent persistence of fetal myoglobin, but this is unproved at this 
time. Elucidation of the mechanisms responsible for the observed changes awaits further study.

The Mechanism of Death in Experimental Pneumococcal Infection. John E. Perry and Leighton E. Cluff,* Baltimore, Md.

Lethal pneumococcal infection in rabbits is characterized by fever, leukopenia, bacteremia, and shock. After intraperitoneal inoculation of $5 \times 10^{8}$ type I pneumococci, $56 \%$ of the animals died within 24 hours and the remainder within 5 days. The rapidity of death could be correlated with the time of onset and degree of bacteremia. None of the rabbits died if treated with penicillin during the first 3 hours of infection. When treatment was delayed beyond 4 hours, the early mortality rate was not affected; however, the animals were protected against late mortality. The difference in mortality during treatment could be correlated with the rapidity in decline of bacteremia. The blood became sterile within 4 hours in those animals which survived; however, bacteremia persisted for 6 to 8 hours in those that died. A similar delay in bacterial killing was observed when penicillin was added to broth cultures of pneumococci more than 4 hours after inoculation. These effects may be related to the emergence of phenotypes which are relatively indifferent to penicillin. Hemodynamic studies revealed a progressively declining cardiac output, an increased peripheral resistance, and a normal venous pressure. During fever, the cardiac output decreased to $78 \%$ of control values and to $54 \%$ just prior to the onset of hypotension. The peripheral resistance increased to $131 \%$ of normal during the febrile period, and to $230 \%$ prior to shock. During the period of hypotension an even greater reduction in cardiac output and increase in peripheral resistance was observed. It is likely that shock during this infection is due to regional pooling of blood and decreased venous return to the heart, resulting in a decreased cardiac output and compensatory increase in peripheral resistance.

The Effect of Insulin-binding Antibodies on the Distribution and Excretion of Insulin in Diabetics with the Nephrotic Syndrome. JoHn R. Petersen, Norman H. Engbring, Norman W. Osher and William W. Engstrom,* Milwaukee, Wis.

The metabolism of insulin, as defined by the distribution and fate of insulin- $\mathrm{I}^{121}$, is altered in insulin-treated subjects by the appearance of insulin-binding antibody. The clinical significance of this antibody in diabetics is uncertain. Decreasing insulin requirements often characterize the diabetic who develops the nephrotic syndrome. It might be anticipated that urinary loss of antibody or insulin, or both, might occur in diabetics with proteinuria. Trichloroacetic acid (TCA)-precipitable radioactivity in plasma and urine was determined at intervals following intravenous injection of insulin- $\mathrm{I}^{\mathbf{1 2 1}}$ in diabetics with and without proteinuria, in nondiabetics with a nephrotic syndrome, and in normal subjects. In normal subjects, insulin was rapidly distributed throughout the extracellular space, and free $\mathrm{I}^{22}$, a product of degradation, was rapidly excreted in the urine. In diabetics whose plasma contained insulin-binding antibody, the greatest amount of insulin was retained within the vascular compartment, and free $\mathrm{I}^{181}$ was excreted slowly. In edematous nephrotic patients, both diabetic and nondiabetic, an expanded insulin pool resulted in a slow turnover rate; however, the effect of insulin antibodies in diabetic nephrotics was to maintain higher blood levels of insulin. Using a salting-out method (modified from Grodsky and Forsham) for selective precipitation of antibody-bound insulin, proteins having insulin-binding capacity were demonstrated in the plasma and urine of diabetic nephrotics. Excretion of TCA-precipitable radioactivity in the urine, antibody-bound or free insulin, correlated with the degree of proteinuria in both diabetics and nondiabetics. Patients without proteinuria excreted no significant precipitable radioactivity. The findings indicate that insulin-binding antibody and probably free insulin are lost in the urine of insulin-treated diabetic nephrotic patients.

Enzyme Activity in the Anatomical Units of Nephrons of Healthy and Acidotic Dogs. Victor E. Pollak and Hermann Mattenheimer, Chicago, Ill. (introduced by Robert M. Kark).

The mechanisms responsible for the renal response to metabolic acidosis are not clearly defined. The possible role of enzymes in the increased ammonia and proton excretion of metabolic acidosis was therefore studied in healthy dogs with normal renal function. Renal biopsies were taken from four dogs after 7 control days and after $\mathrm{NH}_{4} \mathrm{Cl}$ administration for 7 days $(110 \mathrm{mEq} /$ day). A fifth dog served as a control. With $\mathrm{NH}_{4} \mathrm{Cl}$ loading, $\mathrm{NH}_{3}$ excretion increased fourfold (19 to 79 mmoles/day), and proton excretion increased threefold (4 to 14 mmoles/ day). Glutamic oxalacetic transaminase (GOT), glutamic pyruvic transaminase (GPT), glutamic dehydrogenase $(\mathrm{GDH})$, carbonic anhydrase (CA), malate dehydrogenase $(\mathrm{MDH})$, and lactic dehydrogenase (LDH) activities were assayed quantitatively by ultramicrochemical techniques in the individually dissected and weighed glomeruli, proximal and distal convolutions, from $16-\mu$ frozen-dried sections. In healthy dogs GOT and MDH activities were highest in the distal convolutions. GDH, CA, and LDH activities were similar in proximal and distal convolutions. During $\mathrm{NH}_{4} \mathrm{Cl}$ administration, GOT activity increased significantly in proximal convolutions of two dogs, in distal convolutions in one, and in both in one. CA activity increased in proximal convolutions of three dogs. Preliminary observations indicated increased GPT activity in metabolic acidosis. No significant changes occurred in GDH, MDH, or LDH activities. Glutaminase and condensing enzyme activities, measured in homogenates, did not increase with $\mathrm{NH}_{4} \mathrm{Cl}$ administration. In rats, glutaminase activity increased in prolonged metabolic acidosis. Our experiments confirm previous observations that no glutaminase adaptation is necessary 
for the production of the increased $\mathrm{NH}_{3}$ excreted in dogs. They suggest that in dogs transamination reactions might play a role in increased $\mathrm{NH}_{3}$ transfer to amino acids, which could then be deaminated.

Dissociation of Insulin Action in the Forearm of Man. David Rabinowitz and Kenneth L. Zierler,* Baltimore, Md.

During studies of insulin effect in patients with disease of the hypophyseal-adrenocortical axis we noticed that, although there was insulin resistance with respect to glucose uptake by muscle, there was either normal or increased responsiveness to insulin with respect to other metabolic events. Closely related observations have been made in excised rat muscle and adipose tissue by Steinke, Taylor and Renold, Vallance-Owen and Lilley, and Lowy, Blanshard and Phear. These reports leave unanswered the question of whether certain circulating antagonists of insulin prevent its action on muscle but not on fat or discriminate the action of insulin on glucose uptake from its action on free fatty acid (FFA) release. This is a report of our observations on the effect of insulin, administered by local arterial injection, on metabolism of human forearm in situ in five patients with active acromegaly, two with Cushing's disease, and in three normal subjects in whom endogenous growth hormone activity was stimulated by a 66-hour fast. Metabolism was assessed by measurement of forearm blood flow and arteriovenous concentration differences. Glucose and potassium uptake by muscle in response to insulin were less than half normal. Insulin-induced glucose and potassium uptake by adipose tissue was probably less affected but was probably reduced. Insulin-induced inhibition of FFA release from adipose tissue was unchecked in these patients. Indeed, the 66-hour fasted subjects were so exquisitely sensitive that arterial FFA concentrations fell in response to an estimated maximum of $3 \mu \mathrm{U}$ of insulin per $\mathrm{ml}$ recirculating plasma, yet glucose uptake in the forearm, where the local concentration of insulin was 100 times greater, was almost abolished. These studies may dissociate the action of insulin on potassium and on glucose uptake from its action on FFA release. They make a unitary explanation of insulin action difficult.

The Structural Basis for the Action of Neurohypophyseal Hormones upon the Amphibian Urinary Bladder. Howard Rasmussen* AND IRving L. Schwartz,* Madison, Wis. and Cincinnati, Ohio.

When neurohypophyseal hormones are applied to the isolated amphibian urinary bladder (the homolog of the mammalian renal collecting tubule), they produce an increase in its permeability to water. This phenomenon was the basis of the method employed to determine the effect of changes in hormone structure upon biologic activity. Twenty-seven hormone analogs were tested. All produced the same increase in permeability, but the concentration required varied from $10^{-6}$ to $10^{-5} \mathrm{M}$, de- pending upon analog structure. None of them was active in the presence of $10^{-2} \mathrm{M}$ cysteine, which did not inhibit the action of cyclic AMP or theophylline (agents which mimic the hormonal action). Angiotensin at $10^{-4} \mathrm{M}$ was inactive even though it mimicked the action (an oxytocic effect) of these hormones on the rat uterus. The basic requirements for hormone action upon the bladder were: 1) an intact disulfide bond, and 2) a ring of 6 or 7 amino acids (20-23 membered ring). Neither the tripeptide side chain nor any of the charged groups on the ring was essential. Thus, hormone-receptor interaction can be considered a two-step process: 1) the multipoint attachment of hormone to receptor site through hydrogen, hydrophobic, and ionic bonds; and 2) a thiol-disulfide interchange between receptor sulfhydryl and hormonal disulfide. A change of structure in a constituent amino acid of the hormone molecule leads to a change in the affinity of hormone for receptor (step 1). The thioldisulfide interchange (step 2) appears to be the catalytic step that initiates the chain of events which lead to the permeability increase. Previous data on the binding of tritium-labeled hormone to both bladder and kidney tissue, and a current analysis, in Michaelis-Menten terms, of the competition between protons and hormone for an ionized group on the receptor are compatible with this proposal.

Myocardial Metabolism during Epinephrine-induced Necrosis. Timothy J. Regan, Lawrence Troum, Patrick H. Lehan and Harper K. Hellems,* Jersey City, N. J.

In view of the important regulatory role of the sympathetic nervous system on myocardial function, the relevance of its myocardial metabolic alterations in the transition from an inotropic to a pathophysiologic effect has been examined in the intact dog. Left coronary artery infusion of $l$-epinephrine was used to minimize systemic effects. An increase in contractility (first derivative of left ventricular pressure) was achieved with $5 \mu \mathrm{g}$ per minute for 15 minutes in eight animals (a modest dose since $2.5 \mu \mathrm{g}$ per minute was ineffective). Sequential substrate extractions were followed by arterial-coronary sinus sampling. In the presence of a $10 \%$ coronary flow increment $\left(\mathrm{N}_{2} \mathrm{O}\right.$ method $)$, there was a shift in oxidative metabolism as evidenced by a myocardial respiratory quotient rise. An early appearance of excess lactate production, indicative of glycolysis, was associated with a decreased extraction of glucose. Similarly, free fatty acid extraction by the heart diminished. This deficit in available substrate was compensated by extraction of triglyceride, a moiety not usually extracted in significant quantity. To evaluate whether these metabolic effects could be related to the genesis of necrosis and lipid accumulation reported with large doses, the same concentration was extended over 90 minutes in a separate group of eight animals, resulting in changes analogous to ischemic necrosis. Glutamic oxalacetic transaminase and $\mathrm{K}^{+}$rose significantly in the coronary sinus above the arterial levels by about 2 hours, when ventricular 
ectopic activity usually appeared and contractility declined. The metabolic concomitants included a persistence up to 60 minutes of the altered substrate transport observed during the initial positive inotropic effect and then reversion to control levels of free fatty acid extraction and lipid utilization. Assumed that the metabolic events of the first hour are critical to necrosis, increased carbohydrate oxidation may be responsible for triglyceride accumulation during its accelerated transport. Thus, persistent epinephrine activity in modest concentrations may produce cardiac necrosis without coronary flow reduction or relevant ionic alterations. A metabolic genesis related to the interaction of lipid and carbohydrate transport and utilization is suggested.

Adrenocortical Modification of the Interdependence of Calcium and Sodium Reabsorption in the Kidney. Brian H. B. Robinson, Edward B. Marsh, Jr., John W. Duckett, JR. and Mackenzie Walser,* Baltimore, Md.

Administration of adrenal steroids is usually followed by an increase in urinary calcium and a decrease in urinary sodium. However, in other circumstances, calcium reabsorption tends to vary with sodium reabsorption. We have reported that, in dogs, the percentage reabsorption of filtered calcium parallels that of sodium during a variety of diuretic procedures. By contrast, in the hypercalcemia of malignancy, and in dogs (or human subjects) infused with calcium salts, calcium clearance regularly exceeds sodium clearance, often many-fold. This homeostatic response fails to occur in hypercalcemic adrenalectomized dogs; calcium clearance remains less than sodium clearance. In normal human subjects, hourto-hour fluctuations in sodium excretion throughout each 24 hours were accompanied by parallel changes in calcium excretion. During infusion of sodium sulfate, calcium clearance was highly correlated with sodium clearance ( $r=0.87$ for 16 observations in six subjects). By administering mineralocorticoids or aldosterone antagonists, and then varying sodium excretion over a wide range, it is possible to determine whether the action of these steroids on the tubule includes inhibition of calcium reabsorption at any given level of sodium reabsorption. Clearances of sodium, potassium, calcium, magnesium, and inulin were determined during diuresis induced successively by water, mannitol and saline, and sodium sulfate. In one normal subject given fludrocortisone on one occasion and aldosterone on another, average ratios of calcium clearance to sodium clearance were 2.5 and 1.7 , respectively, as compared with 1.2 during a control study. In another normal subject the average ratio was 1.4 while on spirolactone, and 4.0 on fludrocortisone. In a third, the average ratio was 0.4 on spirolactone, compared with 3.5 in a control study. Two Addisonians maintained without mineralocorticoids yielded 0.9 and 1.1, respectively; after deoxycorticosterone acetate the ratios were 1.8 and 1.7. These results indicate that calcium reabsorption is linked with that of sodium; this dependence is altered by mineralocorticoid activity.
Competitive Inhibition of Dibasic Amino Acid Transport in Rat Kidney. Leon E. Rosenberg and Stanton Segal, Bethesda, Md. (introduced by Robert M. Chanock).

Data from renal clearance studies in dogs, and from urinary amino acid patterns in patients with cystinuria have suggested that the dibasic amino acids-lysine, arginine, ornithine, and cystine-share a common renal tubular transport site(s). The present study, utilizing rat kidney cortex slices, was undertaken to define the mechanism of the mutual interference for tubular reabsorption exhibited by these compounds. Isotopically labeled L-lysine, L-arginine, and L-cystine were concentrated against chemical gradients during 30 -minute incubations, and mutual inhibition was demonstrated with equimolar concentrations of L-lysine, L-arginine, and L-ornithine, but L-cystine did not share this common transfer process. The specificity of this shared mechanism was shown by experiments in which L-alanine, L-phenylalanine, and L-histidine failed to inhibit L-lysine uptake, and by studies indicating that transport of glycine, L-phenylalanine, and L-histidine was unaffected by L-arginine. The inhibition of L-lysine by L-arginine was studied during 5- to 120-minute incubation periods, and analysis of the results, using a two-compartment model, suggested an equal reduction in influx and efflux rates in the presence of inhibitor. Utilizing diffusion and Michaelis-Menten kinetics, the inhibition of L-arginine transfer by L-lysine and L-ornithine was shown to result from competitive inhibition for the saturable component of the transport process, and was not related to alteration in physical diffusion rates. In agreement with previous in vivo observations, L-arginine and L-ornithine had a greater affinity for the shared transport site(s) than had L-lysine, while the transport capacity for L-lysine exceeded that for L-arginine. The failure of cystine to share the transport process(es) common to the other dibasic amino acids studied contrasts sharply to observations in cystinuric patients, and suggests that such transport mechanisms are species specific and may be under genetic control.

Coupled Nonionic Diffusion: A Hypothesis to Account for Apparent Changes in Permeability to Weak Electrolytes. Joseph B. Rosenfeld and William B. Schwartz,* Boston, Mass.

Although the transfer of weak electrolytes by nonionic diffusion has been extensively studied, little consideration has been given to the circumstance in which diffusion of both a nonionized acid and nonionized base occurs simultaneously (coupled nonionic diffusion, CND) from a solution in which $\mathrm{pH}$ is not autonomously regulated. Theoretical considerations suggest that transfer of a single weak electrolyte by nonionic diffusion will be limited by the selective loss of the nonionic species and disappearance of the nonionic concentration gradient. However, simultaneous diffusion of a weak acid and weak base in the presence of the ionic members of each conjugate pair (a proton acceptor and donor, respec- 
tively) should lead to continuous regeneration of each nonionic species with progressive loss of the ionic species via CND. In order to test this hypothesis, $100 \mathrm{mM}$ solutions of ammonium chloride ( $\mathrm{pH} 8$ ), sodium bicarbonate, and ammonium bicarbonate were instilled into dog bladders isolated by ureteral and urethral ligation. After instillation of ammonium chloride $\left(\mathrm{pH} \mathrm{8),} \mathrm{NH}_{:}^{+}\right.$ concentration fell by a mean of only $20 \mathrm{mEq}$ per $\mathrm{L}$ in 8 hours, whereas after instillation of ammonium bicarbonate, concentration fell by a mean of $65 \mathrm{mEq}$ per $\mathrm{L}$ $(p<0.001)$. Similarly, after the instillation of sodium bicarbonate, bicarbonate concentration fell by only 10 $\mathrm{mEq}$ per $\mathrm{L}$, whereas in the ammonium bicarbonate experiments it fell by approximately $58 \mathrm{mEq}$ per L $(p<0.001)$. It is proposed that two processes were responsible for the ionic loss from ammonium bicarbonate solutions. In part, ammonium and bicarbonate ions were lost directly along their concentration gradients, just as appears to be the case when each is present individually as the salt of a strong electrolyte. In addition, a large loss occurred indirectly by the continuous transformation of $\mathrm{NH}_{4}{ }^{+}$and $\mathrm{HCO}_{3}^{-}$into $\mathrm{NH}_{3}$ and $\mathrm{CO}$.. These data suggest that the migration of weak electrolytes from a solution in which a constant $\mathrm{pH}$ is not maintained autonomously can be increased by CND, and that this will induce an apparent change in the permeability to each ionic species.

Electrocardiographic and Hemodynamic Observations during Selective Coronary Cineangiocardiography. RICHard S. Ross, Neil Schwartz, Robert A. Gaertwer AND Gottlieb C. Friesinger, Baltimore, Md. (introduced by $\mathrm{A}$. McGehee Harvey).

The electrocardiographic and hemodynamic changes associated with the injection of roentgen-opaque materials into the coronary arteries were studied in man and dogs. The electrocardiogram was recorded during the selective injection of $5 \mathrm{ml}$ of contrast material into each coronary artery in $\mathbf{4 0}$ patients. In all patients with angiocardiographically normal coronary arteries, the $T$ wave vector shifted away from the part of the heart perfused by the injected vessel during the minute after injection. This pattern was altered in the presence of coronary artery disease. In two patients, collateral links between the right and left coronary circulations were demonstrated. In these, after injection into a single artery, right and left coronary $\mathrm{T}$ wave changes appeared sequentially in an order corresponding to the observed flow of contrast material. Serial measurements of left ventricular pressures were made in 12 patients during arteriography. An elevation of left ventricular diastolic pressure and an increase in the "a" wave preceded the onset of anginal pain in two patients. The administration of nitroglycerine was followed by relief of pain and a drop in left ventricular diastolic pressure. These observations probably explain the elevated pulmonary wedge pressure and prominent " $a$ " wave of the apex cardiogram reported in angina pectoris. In open-chested dogs, the electrocardiogram, left ventricular diastolic pressure, coronary blood flow, and myocardial contractile force were recorded during selective injection of contrast material into the coronary arteries. During the first 10 seconds, coronary blood flow, myocardial contractile force, and arterial blood pressure decreased, and $T$ wave changes developed. The $T$ wave changes persisted for 45 seconds, even though arterial pressure and myocardial contractile force had returned to normal by 20 seconds, and coronary blood flow increased to $150 \%$ of control. The increase in coronary flow persisted for 1 minute or longer.

Colloid Osmotic Pressure as a Possible Regulator of Albumin Synthesis. M. A. Rothschild, M. Oratz, E. C. Franklin* and S. S. Schreiber, New York, N. Y.

A colloid osmotic regulatory mechanism was suggested to explain the hypoalbuminemia following dextran infusions. It is suggested that a similar mechanism is responsible for the hypoalbuminemia associated with hyperglobulinemia. To study this hypothesis further, hypergammaglobulinemia was produced in seven female rabbits by the prolonged injection of a polyvalent pneumococcal vaccine. Albumin metabolism was measured with albumin- ${ }^{131}$ during a control period and again after 4 to 6 months of vaccine administration when hypergammaglobulinemia and hypoalbuminemia had appeared. In four rabbits albumin metabolism was restudied after the plasma protein values had returned to normal and again during the anamnestic response. After primary immunization, albumin levels fell from a mean control value of 3.69 to $2.78 \mathrm{~g}$ per $100 \mathrm{ml}$ and $\gamma$-globulin levels rose from 0.75 to $3.80 \mathrm{~g}$ per $100 \mathrm{ml}$. No change in plasma volume occurred. The albumin pool decreased by $21.9 \%$ even though albumin degradation decreased by $23.7 \%$; thus albumin synthesis must have decreased even further to account for this loss in total exchangeable albumin. After the anamnestic response, mean albumin levels decreased from 3.69 to $2.18 \mathrm{~g}$ per $100 \mathrm{ml}$. $\gamma$-Globulin levels rose from 0.84 to $6.07 \mathrm{~g}$ per $100 \mathrm{ml}$, with an increase in the mean plasma volume of $24.5 \%$. Albumin degradation failed to show any change until the plasma albumin concentration had fallen to low values, usually by 7 to 10 days after vaccine administration. Thereafter, albumin degradation decreased $19.5 \%$ below control values. The fractional rate of albumin- ${ }^{121}$ degradation was unaffected. Albumin synthesis decreased by a mean of $23.2 \%$, resulting in a decrease in exchangeable albumin of $27.2 \%$. The production of hypergammaglobulinemia results in hypoalbuminemia and a decreased exchangeable albumin resulting from decreases in albumin synthesis. This alteration in albumin synthesis is consistent with the concept of an osmotic regulatory mechanism controlling albumin synthesis.

Investigation of the Mechanism of Duroziez's Murmur. George G. Rowe* and Skoda Afonso, Madison, Wis.

Radiographic contrast substance has been infused with a constant injection syringe into the femoral artery and 
its motion has been recorded cineangiographically with an intensifier. In the human subject without aortic insufficiency or other abnormal retrograde flow of blood from the central aorta, and in the normal dog, contrast material during systole passes rapidly down the femoral artery; during diastole there is minimal backflow. If the aortic valve of a dog is destroyed so that it becomes incompetent, the amount of femoral arterial backflow increases greatly. In human subjects with aortic insufficiency or patent ductus arteriosus with a large aorticpulmonary flow, there is considerable femoral backflow and a Duroziez's murmur is heard. In one subject with a normal heart but severe anemia, Duroziez's murmur and increased femoral backflow were demonstrated. In general, the degree of femoral backflow seems to correlate with the intensity of the Duroziez's murmur. Present observations seem to afford visual confirmation of a long standing clinical presumption.

Investigation of Possible Chemoreceptors in the Venous and Pulmonary Circulation for the Regulation of Respiration. Herbert A. Saltzman and Herbert $O$. SIEkER,* Durham, N. C.

The presence of respiratory chemoreceptors in the venous or pulmonary circulation is suggested from the ventilatory response to exercise and by the recent demonstration of carotid body-like structures in the lungs. To investigate this possibility, changes in pulmonary (PA) and brachial (BA) arterial blood gases were correlated with minute ventilation $(\mathrm{MV})$ and $\mathrm{CO}_{2}$ elimination $\left(\mathrm{VCO}_{2}\right)$ following release of arterial tourniquets, the rectal infusion of $\mathrm{CO}_{2}$, and exercise in 23 normal subjects. The release of leg arterial tourniquets was followed within a minute by transient increases in mean PA $\mathrm{PCO}_{2}$ of $3.5 \mathrm{~mm} \mathrm{Hg}, \mathrm{MV}$ of $0.6 \mathrm{~L}$ per min, and $\mathrm{BA} \mathrm{PcO}_{2}$ of $1.4 \mathrm{~mm} \mathrm{Hg}$, while blood $\mathrm{pH}$ decreased in a reciprocal manner. The rectal infusion of 1,200 to $2,500 \mathrm{ml}$ of $100 \% \mathrm{CO}_{2}$ in 15 subjects produced statistically significant and prolonged (15 to 35 minutes) increases in mean PA $\mathrm{PCO}_{2}$ of $3.0 \mathrm{~mm} \mathrm{Hg}, \mathrm{MV}$ of $1.6 \mathrm{~L}$ per min, and $\mathrm{VCO}_{2}$ of $50 \mathrm{ml}$ per min, while PA pH decreased $0.02 \mathrm{U}$. The BA $\mathrm{PCO}_{2}$ and $\mathrm{pH}$ did not change significantly from control values. Rectal infusion of comparable volumes of air or $\mathrm{N}_{2} \mathrm{O}$ did not produce significant changes in ventilation. Exercise was associated with increases in $\mathrm{PA} \mathrm{PcO}_{2}$ of $6.0 \mathrm{~mm} \mathrm{Hg}, \mathrm{MV}$ of $16.9 \mathrm{~L}$ per min, and $\mathrm{Vco}_{2}$ of 400 $\mathrm{ml}$ per min, while PA pH decreased $0.02 \mathrm{U}$. The BA $\mathrm{PCO}_{2}$ fell $1.2 \mathrm{~mm} \mathrm{Hg}$, but the $\mathrm{pH}$ was essentially unchanged. Release of leg arterial tourniquets produced brief changes in ventilation in association with blood gas alterations in both central venous and arterial blood. The time sequence of events suggests that the arterial chemoreceptors are responsible for this respiratory change. The increase in central venous $\mathrm{PcO}_{2}$, minute ventilation, and $\mathrm{CO}_{2}$ infusion suggests the presence of chemoreceptors in the venous system or pulmonary circulation which contribute to ventilatory regulation. Such a mechanism may also explain in part the ventilatory response to exercise.
Cardiovascular Actions of Corticosteroids in Normal Subjects and in Patients with Acute Hypotension. M. P. Sambir, M. H. Weil, V. N. UdhojI and L. Rosofr, Los Angeles, Calif. (introduced by Samuel I. Rapaport).

Observations on 11 normal subjects and 10 patients with shock, receiving metaraminol and norepinephrine in graded doses before and after a pharmacologic dose of a corticosteroid, provided no support for the hypothesis that the pressor effects of vasopressor agents are potentiated by corticosteroids. However, a striking hemodynamic effect followed within 0.5 to 3 hours of the administration of corticosteroids. This led us to study the circulatory effects of corticosteroids alone in nine normal subjects and in five patients with shock associated with sepsis (3) and pancreatitis (2) In no instance was a pressor effect observed and intra-arterial pressure and central venous pressure were essentially unchanged. In normal subjects, after the injection of $500 \mathrm{mg}$ cortisol or prednisolone and dexamethasone in equivalent amounts, the cardiac index was uniformly increased from 3.0 (2.4 to 4.1 ) to 3.9 (3.5 to 4.8 ) $\mathrm{L}$ per $\min$ per $\mathrm{m}^{2}$. The change is highly significant $(\mathrm{SED}=0.214, \mathrm{p}<0.01)$. Peripheral resistance declined by an average of $25 \%$. In patients with shock, arterial pressure was 74 (62 to 88) $\mathrm{mm} \mathrm{Hg}$ and cardiac index was 2.3 (1.1 to 3.8) $\mathrm{L}$ per $\min$ per $\mathrm{m}^{2}$. After injection of 20 to $100 \mathrm{mg}$ dexamethasone, no change occurred in one patient, but a marked increase in cardiac output ranging from 23 to $57 \%$ occurred in the remaining four, with a significant reduction in peripheral resistance (10 to 53\%). Pharmacologic doses of corticosteroids increase flow and decrease resistance. These actions oppose the primary defects of reduced cardiac output and detrimental vasoconstriction, the latter particularly when augmented by vasopressor therapy during progression of shock.

Biologic Potential of Transcortin. Avery A. SANDBERG* and W. R. Slaunwhite, JR., Buffalo, N. Y.

Previous observations, based on indirect evidence in human subjects, indicated that transcortin-bound cortisol was 1) biologically inert and 2) unavailable for catabolism. The partial purification of transcortin made it possible to study two of its potentials. In a series of experiments it was shown that cortisol-induced glycogen deposition in the liver of adrenalectomized mice could be completely abolished by the intravenous injection of transcortin. Thus, transcortin rendered cortisol biologically inert. The present study was undertaken to ascertain the second potential of transcortin. Human or rat liver homogenates or microsomes were incubated for $\mathbf{3 0}$ minutes with $\mathrm{C}^{16}$-cortisol (without carrier and as much as $25 \mu \mathrm{g}$ of cortisol) and an excess of an electron donor (TPNH). Almost quantitative reduction of cortisol took place, resulting in pregnane and allopregnane derivatives with human and rat liver, respectively. The addition of human serum albumin did not change the results. In contrast, the presence of as little as 0.06 to 
$0.1 \mathrm{mg}$ of transcortin resulted in the recovery of substantial amounts of unchanged cortisol (ca 30\%). There was a linear relationship between the amounts of cortisol unmetabolized and the concentration of transcortin in the incubation medium. With $2 \mathrm{mg}$ or more of transcortin little reduction of the cortisol nucleus occurred. Incubation with normal plasma or plasma with high transcortin levels (in pregnancy or after estrogen therapy) showed the latter to contain three times as much transcortin as the former. These findings indicate that the affinity for cortisol manifested by transcortin is higher than that shown by the liver enzyme systems responsible for the reduction of the steroid. On the basis of these experiments, the hypothesis is advanced that variations in transcortin levels may lead to abnormal metabolic effects of cortisol, not evident in the commonly measured parameters.

Propertics of Transferable Pyrogen in Experimental Vaccinia Infection. Gerald Sandson, Rose Pavia AND A. I. Braude,* Pittsburgh, Pa.

It is not known if microbial products are responsible for pyrogenicity of serum during infectious fever. To resolve this question, circulating pyrogen in vaccinia infection was inoculated into immune rabbits because the response to microbial, but not endogenous, pyrogen might change. Vaccinia virus, propagated in cultures of rabbit kidney, was selected for study after it proved nonpyrogenic on intravenous injection into normal rabbits. Seventy-two hours after intracorneal or intravenous infection with $10^{5}$ viable virus units, rabbits developed fever reaching $3.5^{\circ} \mathrm{F}$ and lasting 5 days. Intravenous injection of $30 \mathrm{ml}$ serum from febrile rabbits produced immediate monophasic fever reaching $1.5^{\circ} \mathrm{F}$ in 1 hour in all 23 normal rabbits examined. No viable virus was found in pyrogenic serum. In contrast to normals, immune rabbits (recovered from intravenous vaccinia infection) either remained afebrile $(66 \%)$ or exhibited immediate biphasic hyperpyrexia $(33 \%)$ after receiving intravenously $30 \mathrm{ml}$ serum from febrile rabbits infected intravenously. Because immune animals showed similar altered responses to $10^{5}$ viable virus particles, viral antigen was suspected in pyrogenic serum. Influence of antibody on pyrogenicity of virus was next examined by mixing $10^{5}$ viable vaccinia units with serial dilutions of immune serum before intravenous injection into normal rabbits. Virus plus immune serum diluted up to $1: 4$ was nonpyrogenic, but virus plus immune serum diluted $1: 16$ to $1: 64$ produced immediate monophasic fever. Virus plus immune serum diluted beyond 1:64 to 1,024 produced no fever until infection developed at 72 hours. These results demonstrate that fever of vaccinia infection is accompanied by a noninfectious serum pyrogen which produced different responses in immune and nonimmune animals; and that its pyrogenic effect can be duplicated by mixing virus with appropriately diluted immune serum. It is possible, therefore, that fever of experimental vaccinia results from circulating complexes of vaccinia antigen and antibody.
The Nongenetic Nature of the Alteration of Serum Cholesterol Induced by Deoxyribonucleic Acids. J. PhILIP Savitsky, New York, N. Y. (introduced by Louis Leiter).

A single injection of DNA produces in rabbits a decrease in serum cholesterol and phospholipids lasting at least 2 years. Microgram quantities of DNA from homologous and heterologous mammalian tissues are effective in lowering cholesterol levels of animals with high normal values. Chemical, physical, and enzymatic evidence shows that the active material is purified, polymerized, undenatured, 2-stranded DNA. It appeared important to determine whether the prolonged alteration of serum cholesterol regulation reflected the introduction of specific, transferable, genetic information into adult mammals. A standardized assay of DNA physiologic activity was developed: A citrate-saline solution of $150 \mu \mathrm{g}$ DNA was injected intravenously into each of ten normal rabbits with appropriate controls (citrate inhibits the plasma deoxyribonuclease). Serum cholesterol and phospholipids were determined prior to the injection and at monthly intervals for 6 months thereafter. Using this assay, three groups of experiments were performed. 1) Eighteen DNA preparations from five mammalian species and seven normal tissues have been tested. The preparations lowered the serum cholesterol with no specificity observed as to species or tissue source of the DNA. 2) Individual normal rabbits were selected which maintained levels of serum lipids for 6 months with minimal variation. The serum cholesterol levels of the "high cholesterol" group averaged fourfold that of the "low cholesterol" rabbits. DNA prepared from both groups was equally effective in the assay. 3) Normal rabbits were again selected for minimal variation of serum cholesterol. Leukocytes were obtained from these animals and DNA prepared. Each rabbit was injected with its own DNA. The autologous DNA effectively lowered the serum cholesterol and phospholipids, comparably to all other preparations. The data indicate that the altered serum cholesterol regulation induced by heterologous, homologous, and autologous DNA is not associated with the transfer of specific, genetic information.

Metabolism and Membrane Transport of Erythrocyte Stroma. Stanley L. Schrier and Lydie S. DoAk, Palo Alto, Calif. (introduced by Frederic L. Eldridge).

Membrane transport frequently involves movement against a gradient and therefore requires energy and specific transport systems. Studies on metabolism and transport systems of erythrocyte membranes (stroma) were undertaken with the intent of providing information on mechanisms of membrane transport and intracorpuscular red blood cell defects. Stroma was prepared by dialyzing normal human erythrocytes against increasingly hypotonic saline solutions. The resulting membranes were white, homogenous, biconcave discshaped, without rents, and contained approximately $1.5 \%$ of hemoglobin by dry weight (or less than $0.03 \%$ of the original hemoglobin). Studies of carbohydrate metab- 
olism in stroma showed that stroma did not utilize glucose, fructose, inosine, adenosine, ribose, or ribose1-phosphate, even when appropriate cofactors were added. However, ribose-5-phosphate (R5P) was promptly metabolized to ribulose-5-phosphate (RU5P) indicating presence of pentose phosphate isomerase. We searched for further products of R5P metabolism because conversion of R5P to glyceraldehyde-3-phosphate (GA3P) would indicate the presence of a triple enzyme sequence of the pentose phosphate pathway in stroma: pentose phosphate isomerase, pentose phosphate epimerase, and transketolase. Our results demonstrated that stroma does catalyze the conversion of R5P and GA3P. Kinetic studies showed that appearance of GA3P followed production of ketopentose phosphate and that the rate of both reactions depended on stroma concentration. In addition, specific assays demonstrated relatively high concentrations of the enzyme glyceraldehyde-3-phosphate-dehydrogenase (GA3PD) in stroma. Our studies have shown the presence in red cell membranes of a metabolic pathway which usually involves three sequential enzymes in the pentose phosphate pathway leading to the production of GA3P from R5P. The presence of GA3PD in stroma together with a system for production of its substrate, GA3P, could result in an energy-yielding oxidative phosphorylation in the membrane, and thus account for inorganic phosphate transport, partly explain potassium transport, and perhaps other energy-requiring systems.

Effect of Calcium and of Phosphate Intake on Radiostrontium Absorption in Mice. Robert Schwartz, Cleveland, Ohio (introduced by George J. Gabuzda, Jr.).

Although strontium and calcium are metabolized similarly by bone, these ions are transported differently by kidney and by intestine. Absorption of strontium is conditioned by the calcium content of the diet. Since little is known about the influence of dietary phosphate, the effects of independently varying calcium and phosphate intakes on radiostrontium absorption were studied in mice. The progeny of mice, fed a vitamin D-free diet containing adequate calcium and phosphate, were weaned at 18 days. Groups of 10 to 15 litter mates were fed ad libitum synthetic diets, the calcium and phosphate contents of which were varied. Radiostrontium $85\left(\mathrm{Sr}^{85}\right)$ was given after 18 to 21 days. Whole body counting of each mouse was then performed daily for 1 week. Body content of $\mathrm{Sr}^{85}$ given p.o. was inversely related to dietary calcium level when the diet contained adequate phosphate. In the absence of phosphate, $\mathrm{Sr}^{\mathrm{s}}$ retention was increased. For example, $\mathrm{Sr}^{85}$ retention at 24 hours was 18 and $62 \%$ when the diets contained 0.94 and $0.02 \mathrm{~g}$ $\%$ phosphate, respectively, and $1.2 \mathrm{~g} \%$ calcium. These effects were evident by 5 hours after administration of $\mathrm{Sr}^{85}$. Approximately $80 \%$ of isotope given intravenously was retained after 24 hours. Altering dietary calcium and phosphate had only minimal effects; slightly less isotope was retained when the diet contained little phosphate but adequate calcium. The rates of loss of isotope after the first 24 hours were similar for the two routes of administration. These were not influenced by giving vitamin $\mathrm{D}$, although feeding the low phosphate diet caused mild chemical rickets. The differences noted between the oral and intravenous experiments indicate that absorption of $\mathrm{Sr}^{85}$ in mice is impaired by the presence of phosphate in the diet, which presumably forms insoluble salts with strontium.

The In Vitro Metabolism of 6-Mercaptopurine in Human Leukemic Leukocytes. JAMES L. ScotT AND Joseph V. Marino, Los Angeles, Calif. (introduced by John S. Lawrence).

In animal tumor cells and bacteria, 6-mercaptopurine (6-MP) has been found to inhibit de novo purine synthesis, specifically affecting the conversion of other purines to adenine prior to its utilization for nucleic acid synthesis. In the present studies evidence of this effect was sought in leukocytes isolated from the blood of patients with acute leukemia. De novo purine synthesis was measured by the incorporation of formate- $\mathrm{C}^{14}$ into the nucleic acid purines. After incubation with a pharmacological concentration $\left(6 \times 10^{-4} \mathrm{mmoles} / \mathrm{ml}\right)$ of 6-MP, incorporation was lower than control values in three cell populations, unchanged in two, and higher in three others. Adenine and guanine incorporation were influenced equally, so that the effect on adenine utilization was not a specific one. These in vitro effects could not be clearly related to clinical responsiveness to 6-MP. To clarify these results, $\mathrm{C}^{\mathbf{1 4}}$-labeled $6-\mathrm{MP}$ was incubated with acute leukemic leukocytes and the metabolic products identified by radiochromatography. Conversion of 6-MP to physiological purines and the utilization of these compounds for nucleic acid synthesis by the cells of patients with 6-MP-resistant leukemia were observed. This capability may represent one mechanism of drug resistance. Since preformed purine is utilized in preference to that synthesized by the de novo pathway, inhibition of purine synthesis by 6-MP may reflect drug inactivation in these cells.

Periodic Hemodialysis for the Rehabilitation of Patients with Terminal Uremia. Belding H. Scribner,* Seattle, Wash.

A new, pumpless, low temperature hemodialysis system and a technique of long-term cannulation of blood vessels have been developed and perfected to the point where it is now possible to restore to good health selected young adults with chronic kidney disease who would otherwise die of terminal uremia. The first four patients were begun on a weekly hemodialysis during the winter of 1960. One of these patients, with a creatinine clearance of about $1 \mathrm{ml}$ per min, continues to work full time. Two others, who have been anuric for the last 18 months, lead comfortable sedentary lives. The fourth died of a myocardial infarction after a year of hemodialysis therapy. Hypertension, which became malignant in one patient, has been satisfactorily controlled solely by a low salt diet and ultrafiltration of extracellular fluid during 
dialysis. About a year ago two of the four patients developed a slowly progressive peripheral neuropathy and all complained of uremic symptoms prior to each weekly dialysis. Switching from a long weekly dialysis to 12 to 16 hours of dialysis twice a week has resulted in elimination of predialysis symptoms and improvement in neuropathy. In the last 8 months four additional patients have been added to the program. Each was carefully selected as being an emotionally stable, mature, cooperative adult under the age of 40 who was free of serious complications of hypertension. Each was invalided by terminal uremia at the time treatment was begun. Hemodialysis once or twice weekly has restored each to good enough health to return to work; one patient married recently. All patients require weekly transfusions to prevent severe anemia. Accumulation of iron may represent a potential threat. Exhaustion of cannula sites is another potential problem, but recent improvements in cannula design may have solved this problem. It is concluded that selected young adults with terminal uremia can be restored to good health with periodic hemodialysis.

A Mechanism of Action of Colchicine in Acute Gouty Arthritis. J. E. SeEgmiller, R. Rodney Howell and Stephen E. Malawista, Bethesda, Md. (introduced by Gordon M. Tomkins).

A role for uric acid in the genesis of acute gouty arthritis has been generally denied, and the mechanism of action of the specific therapeutic agent, colchicine, has remained an enigma. We have recently shown that the intra-articular injection of microcrystalline sodium urate produces an inflammatory response in both gouty and nongouty individuals which is in some cases indistinguishable from spontaneous acute gouty arthritis. A constant feature of both the induced and the spontaneous inflammatory reaction is a phagocytosis of the urate crystals and production of large quantities of lactic acid by the leukocyte-rich effusion obtained from the involved joint when incubated in vitro. We have now observed that the amount of inflammatory reaction and phagocytosis is substantially diminished by treatment of patients with therapeutic amounts of colchicine prior to injection of the crystals. Therapeutic levels of colchicine also resulted in a diminished phagocytosis by leukocytes when tested in vitro, as shown by direct count of intracellular crystals as well as a diminished destruction of microcrystalline sodium urate-6- $\mathrm{C}^{14}$ by leukocytes to yield $\mathrm{C}^{14} \mathrm{O}_{2}$. Therapeutic amounts of colchicine administered in vivo also had a marked effect on the metabolism of leukocytes. The threefold increase in $\mathrm{C}^{14} \mathrm{O}_{2}$ production from glucose$1-C^{14}$ by leukocytes, which is induced by phagocytosis in vitro, was completely prevented by plasma from the same subject after receiving a therapeutic dose of colchicine. We propose that the diminished metabolic activity of the leukocyte in response to colchicine results in a diminished phagocytosis and acid production which serve to interrupt the cycle of new crystal deposition, inflammatory response, phagocytosis of crystals, and increased acid production that seems to be essential for continuation of the acute attack of gouty arthritis.

Observations on Cataract Formation in the Newborn of Pregnant Rats Fed a High Galactose Diet. Stanton Segal and Howard Bernstein, Bethesda, Md. (introtroduced by Jacob Robbins).

The toxic effects of maternally ingested galactose on the human fetus with congenital galactosemia are as yet undetermined. Since cataract formation occurs in galactosemia and is a known manifestation of galactose toxicity in rats, experiments were performed to evaluate in utero effects of this sugar by ascertaining the incidence of cataracts in the newborn. A diet of $40 \%$ galactose was started in pregnant Sprague-Dawley rats at different times from mating until 3 days before term and continued to delivery. Lenses of the newborn were examined visually and by slit-lamp. All animals in 15 litters had nuclear cataracts in various stages of opacification. Histopathologic examination revealed degeneration of central lens fibers similar to early nuclear galactose cataracts in humans. Galactose-1-C $\mathrm{C}^{14}$ oxidation to $\mathrm{C}^{14} \mathrm{O}_{2}$ in vitro was assayed in liver mince from 14- and 20-day fetuses and from 1,20 , and 60 day old rats. In the 14-day fetus, $9 \%$ per $100 \mathrm{mg}$ of liver was oxidized in 1 hour; in the older animals, the rate was 2 to $3 \%$. The higher oxidation by the youngest liver was related to glucose oxidation, since the ratio of $\mathrm{C}^{14} \mathrm{O}_{2}$ from galactose and from glucose$1-C^{14}$ was 1.2. This increased to 2.5 in the 20-day rat, indicating a maturation of galactose conversion to glucose. Although fetal liver can metabolize galactose, the results indicate that maternal ingestion of large amounts of galactose is toxic to the fetus in utero even after as little as 3 days of feeding. The ease of cataract formation in the fetus may be due to a lower total capacity of the liver to metabolize galactose due to its small size. This study indicates that in the human galactosemic fetus, normal galactose intake by the mother may be toxic and suggests the need for galactose-free diets for pregnant mothers who have had galactosemic children.

\section{A Novel Metabolic Pathway of Fat Absorption in the Intestinal Mucosa. JOHN R. SeNIOR AND KURT J. ISSELBACHER,* Boston, Mass.}

A new pathway has been demonstrated whereby the major products of fat digestion may be resynthesized to triglycerides in the intestinal epithelial cells during lipid absorption. After the intraluminal hydrolysis of the insoluble dietary triglycerides to free fatty acids and lower glycerides, especially monoglycerides, these products readily associate with conjugated bile salts to form ultrafine aggregates, or micelles. Significant quantities of monoglycerides are absorbed, and we have shown their incorporation into triglycerides, the main component of lymph chylomicrons. An enzyme has been found in gut mucosal cells that catalyzes the direct recombination of monoglycerides and activated fatty acids to diglycerides, which then are esterified to triglycerides. This mechanism for the direct esterification of monoglycerides has not 
been found in the other tissues in which active triglyceride synthesis occurs, and therefore may be unique to the intestinal mucosa. Utilizing techniques developed recently in this laboratory for fractionation of jejunal epithelial cells, this enzyme, designated monoglyceride acylase, has been shown to be localized in highest specific activity in the microsomes, as has been found also for the long chain fatty acid activating enzyme, thiokinase. By means of chemically-synthesized palmityl-1- $\mathrm{C}^{14}-\mathrm{CoA}$ and glycerol-labeled monopalmitin- $\mathrm{C}^{\mathbf{1 4}}$, very rapid and impressive condensation of the activated fatty acid and monoglyceride was demonstrated. Monoglycerides were found to be better acceptors of the palmityl-CoA than L- $\alpha$-glycerophosphate, previously considered the "natural" acceptor. No significant amounts of phosphorylated intermediates were detected, and neither ATP nor other cofactors were required. In summary, the enzymes necessary for triglyceride synthesis are associated with the microsomes, a fraction principally derived from membranes of the endoplasmic reticulum, within which lipid droplets accumulate during fat absorption, as seen by electron microscopy. The observed monoglyceride "shunt" to triglyceride formation appears to be a mechanism by which considerable amounts of fat can be made available rapidly for export to the rest of the body via lymph chylomicrons.

Increased Susceptibility to Pyelonephritis during Acute Hypertension Induced by Vascoconstrictor Agents. Alvin P. Shapiro and Roger K. Jones, Pittsburgh, $\mathrm{Pa}$. (introduced by Jack D. Myers).

Increased susceptibility to hemat genous pyelonephritis was demonstrated previously in rats made hypertensive by renal artery constriction or deoxycorticosterone. Chronic hypertension however may injure the kidney and favor renal infection. To determine the effects of hemodynamic changes alone, susceptibility to pyelonephritis during an acute hypertensive episode was evaluated. Ten $\mu \mathrm{g}$ of angiotensin given intravenously to normotensive rats elevated blood pressure to $172 \pm 14 \mathrm{~mm} \mathrm{Hg}$ within 2 minutes, with return to baseline by 5 minutes. Three groups then were studied: group A (44 rats) received $10 \mu \mathrm{g}$ of angiotensin i.v. in $0.5 \mathrm{ml}$ of broth containing $10^{8}$ Escherichia coli per $\mathrm{ml}$; group B (45 rats), E. coli in broth alone; and group C (16 rats), angiotensin in sterile broth. Rats were sacrificed at 1, 4, and 24 hours, and 1 and 2 weeks after injection. Kidneys from rats in group $\mathrm{C}$ were sterile and displayed no gross or microscopic alterations. At 1 and 4 hours, numbers of $E$. coli in kidneys of groups $A$ and $B$ were not different. However, at 24 hours, 1 , and 2 weeks, the mean logarithms of bacteria per $\mathrm{g}$ of kidney in group $\mathrm{A}$ were 5.768, 4.554, and 3.706 , respectively; in group $B$ at these periods the mean logs were $3.811,2.130$, and $1.020(p<0.001)$. Bacteremia did not account for the differences. Gross pyelonephritic lesions were present in kidneys of group $\mathrm{A}$ as early as 24 hours (11\%), and were 54 and $67 \%$ at 1 and 2 weeks, respectively. In group B, lesions were absent at 24 hours and present in only $8 \%$ at 1 and 2 weeks $(p=0.001)$. Susceptibility similarly was increased with $10 \mu \mathrm{g}$ of norepinephrine plus $E$. coli, but to a lesser degree. Only $30 \%$ of kidneys in these rats demonstrated gross lesions at 1 and 2 weeks, and increase in renal bacteria was only tenfold. Enhanced susceptibility to pyelonephritis during transient elevation of blood pressure by vasoconstrictor agents supports a primary role for hypertension in the pathogenesis of pyelonephritis.

Prognosis of Tropical Sprue. T. W. Sheehy, E. Perez AND M. H. Floch, San Juan, Puerto Rico (introduced by William H. Crosby).

The prognosis of tropical sprue was evaluated by studying the course of the disease and the fate of the jejunal lesion in 46 Puerto Rican patients. These patients were divided into two groups. Group I consisted of 20 patients with an initial attack of classical sprue, and group II consisted of 26 sprue patients already treated for periods ranging from 3 to 5 years. Both groups were hospitalized on a metabolic ward and subjected to biochemical absorption studies $\left(\mathrm{D}^{+}\right.$xylose, vitamin $\mathrm{A}$, carotene, $\mathrm{Co}^{80}$-vitamin $\mathrm{B}_{12}$ ), measurement of fecal fat, small bowel roentgenological motor meal, and jejunal biopsy. These studies were repeated every 6 months for a 2 to 3 year period. All patients were treated with folic acid or folic acid and vitamin $B_{12}$. The immediate therapeutic results were partial amelioration of intestinal symptoms, elimination of megaloblastic anemia, and clinical improvement. Intestinal function, as determined by chemical, histological, and roentgenological methods, was restored in 9 of the group I patients (45\%) after 6 to 36 months of therapy. Seven patients from group II (27\%) recovered after from 3 to 6 years of therapy. The histopathological changes, small bowel roentgenological pattern, and chemical tests correlate well in tropical sprue. Intestinal absorption is restored only when the jejunal lesion heals. In contrast to celiac disease, the jejunal lesion of tropical sprue is sometimes reversible, and subtotal villous atrophy is rare ( 2 of 46 cases). Moreover, even when subtotal villous atrophy is found, the villous pattern can revert to normal. Persistently impaired absorption of radioactive vitamin $B_{12}$, even in those patients with recovery of all the other indices of intestinal function, suggests permanent derangement of vitamin $B_{12}$ absorption in those who develop chronic sprue.

Arbovirus Infections of Puerto Rican Children with Obscure Neurological Syndromes. A. Shelokov, C. J. Gibbs And D. Mendez-Cashion, Bethesda, Md. and San Juan, Puerto Rico (introduced by Joseph E. Smadel).

A serological survey performed on specimens from 145 healthy adult Puerto Ricans showed that $16 \%$ had complement-fixing antibodies against $B$ group arthropodborne viruses, $3 \%$ had antibodies against $\mathrm{A}$ group, while none showed clearly positive reactions with $\mathrm{C}$ group antigens. Ninety-four sets of paired sera obtained from Puerto Rican children with obscure neurological syndromes were available for more definitive testing. The 
convalescent specimens from all of the cases were tested with complement-fixing antigens representative of the three groups-i.e., Eastern and Western (A) ; St. Louis, Ilheus, Dengue-2 (B); and Apeu (C)-prepared by Casals' technique. Only eight of these convalescent sera reacted with any of the antigens. Seven of the eight cases were hospitalized with convulsions, and the other with meningitis. One of the infants with febrile convulsions and bronchopneumonia displayed a diagnostic rise in Ilheus antibody. Another infant who had a clinical diagnosis of purulent meningitis, and the only patient among the eight with pleocytosis, developed during convalescence antibodies against members of both $\mathrm{A}$ and $\mathrm{C}$ groups. The serum obtained on the fourth day of disease was negative with Eastern, Western, and Apeu, while that obtained on day 18 had a titer of $1: 32$ with both members of the A group and also with Apeu. Of the remaining six patients in the selected group, two had low levels of Apeu antibodies on admission and these did not change significantly during convalescence. In contrast, the other four had no detectable antibodies early in the disease, but developed titers of $1: 4$ to $1: 8$ against Apeu during convalescence. Two of these 4 cases displayed hemiplegia during the acute illness. It is evident from the serological studies that Apeu virus, or a related agent, is producing central nervous system disease in Puerto Rican children. In addition, members of the A and B groups of arbovirus are producing infections among inhabitants of Puerto Rico.

Effect of Acetate and Pyruvate on Metabolism of Glucose- $U-C^{14}$ and Palmitate-1-C $C^{14}$ in Isolated Perfused Rat Heart. J. C Shipp, L. Opie and J. Evans, Boston, Mass. and Gainesville, Fla. (introduced by S. P. Martin).

The isolated rat heart perfused in a closed system with bicarbonate buffer permits a direct measure of the uptake and certain cellular fates of labeled substrates; the $\mathrm{C}^{14} \mathrm{O}_{2}$ formed indicates substrate oxidation. In this study hearts from rats fasted overnight were used. Palmitate-1-C ${ }^{\mathbf{1 4}}$ ( $0.5 \mathrm{mmole} / \mathrm{L}$ in $0.5 \%$ albumin) was readily extracted by the heart and about $80 \%$ of the uptake was recovered as $\mathrm{Co}_{2}$ and "tissue fatty acids." The addition of glucose ( $5 \mathrm{mmoles} / \mathrm{L}$ ) increased the uptake and the incorporation of palmitate label into $\mathrm{CO}_{2}$ and into "tissue fatty acids." Sodium pyruvate $(5 \mathrm{mmoles} / \mathrm{L})$ did not increase the uptake or fate of palmitate, and in certain conditions palmitate oxidation was reduced. Sodium acetate ( 5 mmoles/ L) did not affect the uptake, but reduced the label in $\mathrm{CO}_{2}$ while increasing the label in "tissue fatty acids." Glucose-U-C ${ }^{14}$ (5 mmoles/L) was extracted and recovered, primarily in $\mathrm{C}^{14} \mathrm{O}_{2}$, lactate, and glycogen. The addition of palmitate $(0.5 \mathrm{mM}$ in $0.5 \%$ albumin $)$, sodium acetate $(5 \mathrm{mmoles} / \mathrm{L})$, or sodium pyruvate $(5 \mathrm{mmoles} / \mathrm{L}) \mathrm{did}$ not affect glucose uptake, but the oxidation of glucose-U$\mathrm{C}^{14}$ to $\mathrm{C}^{14} \mathrm{O}_{2}$ was reduced to 18,8 , and $36 \%$ of the control, respectively. The sparing effect on glucose oxidation of all three subtrates was associated with more lactate formation, higher net glycogen and more incorporation of glucose label into glycogen. These observations strongly suggest that palmitate, acetate, and pyruvate are oxidized by the heart in preference to glucose, and that the control for substrate oxidation is at the pyruvateacetyl-CoA level.

A New Method for Measuring Minimum In Vivo Concentrations of Factor VIII Applied in Distribution and Survival Studies. N. RAPHAEl Shulman,* Victor J. Marder and Merilyn C. Hiller, Bethesda, Md.

Small amounts of plasma ( 0.5 to $25.0 \mathrm{ml}$ ) containing normal Factor VIII concentrations were injected into severe hemophiliacs. After a mixing period, blood was drawn for 2-stage prothrombin consumption determinations. In vivo Factor VIII concentrations from 0.02 to $0.8 \%$ of normal, produced by dilution of Factor VIII in the hemophiliac's plasma, were correlated with per cent prothrombin consumed (15 to $85 \%$ ). Standard curves prepared in this way were identical in five different hemophiliacs, and could be used to measure unknown in vivo Factor VIII concentrations with aproximately $\pm 20 \%$ error. This sensitive technique permitted measurement of Factor VIII survival in vivo for 12 half-lives over a 5-day period. Previously available methods were useful for, at most, 6 half-lives, covering less than 2 days. Prolonged survival measurements gave decay curves with two exponential components which were not previously discernible. The initial component had a $t_{1}$ of 4 to 5 hours (primarily extravascular diffusion); the second component had a $t_{\frac{1}{2}}$ of 9 to 11 hours (degradation and back diffusion). These two components, which did not vary with the amount of Factor VIII administered, indicated an extravascular compartment approximately 1.5 times the plasma volume. Fresh plasma, infused at rates of $3,000 \mathrm{ml}$ per $50 \mathrm{~kg}$ body weight in 6 hours, gave Factor VIII concentrations of only 40 to $50 \%$, and markedly expanded the hemophiliac's plasma volume. Factor VIII concentration fell below the therapeutic 30\% level within the next 7 hours. A recently available commercial Fraction I preparation (Merck Sharp \& Dohme, $1 \mathrm{~g} / 50 \mathrm{ml}$ ), infused at rates of $1,500 \mathrm{ml}$ per $50 \mathrm{~kg}$ body weight in 3 hours, gave Factor VIII concentrations greater than $100 \%$ without significantly increasing the plasma volume. Therapeutic levels greater than $30 \%$ persisted for the next 25 hours. The data support recent clinical observations suggesting that Fraction $\mathrm{I}$ is better than plasma for treating hemophilia.

Operation of the Renal Countercurrent Mechanism during Water Diuresis. Miles H. Sigler, Philadelphia, $\mathrm{Pa}$. (introduced by Earl S. Barker).

The operation of the renal countercurrent mechanism during water diuresis in the dog was studied by means of kidney tissue analysis. Kidneys were removed from eight dogs during maximal water diuresis (urine osmolality 50 to $200 \mathrm{mOsm} / \mathrm{kg}$ ) and frozen in liquid nitrogen (removal and freezing time 15 to 30 seconds). The same was done in seven moderately dehydrated dogs (osmo- 
lality 900 to $1,700 \mathrm{mOsm} / \mathrm{kg}$ ). Sections of inner papilla, outer papilla, outer medulla, and cortex were analyzed. Papillary sodium concentration was reduced in water diuresis $(128 \mathrm{mEq} / \mathrm{L})$ as compared to the hydropenic group $(230 \mathrm{mEq} / \mathrm{L})$ with a consequent diminution in the papillary:cortex sodium gradient. However, total sodium content of the inner papilla of the hydropenic group (1.45 $\pm 0.29 \mathrm{mEq} / \mathrm{g}$ dry solids) was only slightly greater than the sodium content in the diuretic group $(1.28 \pm 0.16 \mathrm{mEq} / \mathrm{g}$ dry solids). The difference between groups was not quite statistically significant $(p=0.06)$. Increase in inner papillary water content in the diuretic group ( 90.4 vs $85.1 \%$ in hydropenia) appears to be a primary factor in the decrease in papillary sodium concentration. These was no evident correlation between GFR, serum sodium level, state of water balance, and measured papillary sodium content. During water diuresis there was a slight increase in papillary $\mathrm{K}^{+}$content, with a papillary:cortex gradient of $1.18 \pm 0.18$ (vs 0.96 \pm 0.02 in hydropenia, $\mathrm{p}<0.02$ ). Water diuresis resulted in marked depletion of papillary urea (17.24 $\mu$ moles/g wet tissue vs $220 \mu$ moles in hydropenia). This occurred in conjunction with an increase in the urea:creatinine clearance ratios. It appears that 1) antidiuretic hormone has little direct influence on the countercurrent multiplier as regards the establishment of a high papillary sodium content, and 2) diminished papillary osmolality during water diuresis is due primarily to increased water content and depletion of papillary urea.

The Role of the Liver in Clearance of Blood Coagulation Product $I$. Theodore H. Spaet,* New York, N. Y.

The present study is an extension of previous investigations designed to test the hypothesis that in vivo blood fluidity is preserved by a clearance mechanism whereby clotting intermediates are removed during circulation through certain tissues or organs. Experimental animals were Sprague-Dawley male rats, and biological reagents were of rat origin. Product I was prepared by reacting adsorbed plasma with serum in the presence of calcium ion. Intravenous product I injections failed to produce a drop in plasma fibrinogen in intact animals, but similar injections accompanied by clamping of the hepatic blood supply were followed by significant depression of fibrinogen levels. Saline injection with hepatic artery ligation and product $I$ injection with renal vessel ligation both failed to produce defibrination in control animals. Marked reduction of product $I$ activity resulted from perfusion of this reagent through isolated rat livers, but activity was preserved after perfusion through the hind legs. Reticuloendothelial blockade with carbon produced minimal and inconclusive depression of product I inactivation in the perfused liver, but addition of small amounts of the heparin-neutralizing drugs, protamine or Polybrene, to the perfusate almost completely blocked hepatic inactivation of product I. When perfused product I was mixed with control material in vitro, no anticoagulant effect was noted. Perfusion through the liver of pre- cursor reagents showed that plasma thromboplastin component was inactivated, but antihemophilic factor, factors V, VII, and X were unaffected. Earlier studies showed that blood thromboplastin is cleared by the reticuloendothelial system. The present data suggest that product $I$ is handled by a different mechanism, which may involve participation of hepatic heparin.

Pulmonary Function after Experimental Autologous Pulmonary Emboli. Myron Stein, Israel Alkalay and Israel Bruderman, Boston, Mass. (introduced by Stanford Wessler).

After release to the lungs of endogenous thrombi from the peripheral veins of the $\operatorname{dog}$, arterial $\mathrm{O}_{2}$ saturation, plasma-dissolved oxygen after ventilation with 99.6\% $\mathrm{O}_{2}$, and cardiac output are decreased, while respiratory rate, minute volume of ventilation, and arterialalveolar (a-A) $\mathrm{PcO}_{2}$ differences are increased. Further studies demonstrate that postembolic decreases in lung compliance $\left(\mathrm{C}_{L}\right)$ and increases in mean frictional resistance are transient, returning to control levels 15 to 30 minutes after embolization. Measurements of lung volumes did not vary significantly from pre-embolic determinations. The a-A $\mathrm{PCO}_{2}$ difference, which was not significantly augmented by bronchodilator drugs, was completely eliminated by inhalation of $2.5 \% \mathrm{CO}_{2}$. Acute decreases in plasma-dissolved $\mathrm{O}_{2}$ concentration during ventilation with $99.6 \% \quad \mathrm{O}_{2}$ were not reversed by positive pressure ventilation until 3 days after embolization. a-A $\mathrm{Pco}_{2}$ differences also returned to control levels within 4 days after emboli release. Necropsy examination, however, revealed persistent massive pulmonary embolization. The arterial blood $\mathrm{O}_{2}$ saturation studies indicate that acute pulmonary embolism induced a perfusion defect consistent with a right-to-left shunt. Flow through atelectatic lung seems unlikely since 1 ) no correlation exists between $\mathrm{CL}_{\mathrm{L}}$ decrease and shunt size, 2) postembolic decrease in lung volumes did not occur, and 3) ventilation with positive pressure $\mathrm{O}_{2}$ failed to produce a rise in plasma-dissolved $\mathrm{O}_{2}$ immediately after embolization. Changes in lung mechanics appeared to be related to endogenous release of a bronchoconstrictive substance. The return of arterial blood $\mathrm{O}_{2}$ saturation and a-A $\mathrm{PCO}_{2}$ differences to control levels 4 days after embolization could not be attributed to lysis of emboli or pulmonary edema or infarction. The anatomic location of the shunt remains to be demonstrated.

Effects of Salt Depletion on the Concentrating Operation in Hydropenic Man. R. M. Stein, B. H. Levitt, J. G. Porush, G. M. Eisner, M. H. Goldstein And M. F. Levitt,* New York, N. Y.

Solute diureses were produced with hypertonic mannitol infusions in maximally hydropenic subjects on high salt diets (18 $\mathrm{g} \mathrm{NaCl} /$ day $)$ and after 7 days of salt restriction $(0.8 \mathrm{~g} \mathrm{NaCl} /$ day $)$. No difference in maximum $\mathrm{U}_{\mathrm{osm}}$ was noted but $\mathrm{T}^{\mathrm{c}} \mathrm{H}_{2} \mathrm{O}$, when plotted against solute clearance $\left(\mathrm{C}_{\mathrm{osm}}\right)$, rose more 
slowly and attained a lower maximum in the salt-free group. In both groups $\mathrm{T}^{\mathrm{c}} \mathrm{H}_{2} \mathrm{O}$ tended to stabilize between a $\mathrm{C}_{0 \mathrm{sm}}$ of 14 to $25 \mathrm{ml}$ per minute. At $\mathrm{C}_{0 \mathrm{sm}}$ greater than $25 \mathrm{ml}$ per minute $\mathrm{T}^{\mathrm{c}} \mathrm{H}_{2} \mathrm{O}$ fell below maximum in both groups, but hypotonic urine was not observed with levels of $\mathrm{C}_{\text {osm }}$ as high as 40 to $50 \mathrm{ml}$ per minute. Control $\mathrm{Na}$ excretion $\left(\mathrm{U}_{\mathrm{Na}} \mathrm{V}\right)$ averaged 15 $\mu \mathrm{Eq}$ per minute in the salt-free subjects and $150 \mu \mathrm{Eq}$ per minute in those on the high salt diet. As $\mathrm{C}_{\text {osm }}$ increased to $8 \mathrm{ml}$ per minute, $\mathrm{Na}$ excretion rose slightly, but thereafter a more rapid increase was noted in both groups. Salt restriction produced a variable reduction in GFR, averaging 14 per cent. When GFR was increased at low levels of solute clearance ( 4 to $7 \mathrm{ml} / \mathrm{min}$ ) in the salt-free group by the administration of aminophylline, $\mathrm{T}^{\mathrm{c}} \mathrm{H}_{2} \mathrm{O}$ rose to levels obtained in the high salt group. Aminophylline administered at $\mathrm{C}_{\mathrm{osm}}$ greater than $12 \mathrm{ml}$ per minute in both groups produced no appreciable change in $\mathrm{T}^{\mathrm{c}} \mathrm{H}_{2} \mathrm{O}$ despite comparable increments in GFR and $U_{\mathrm{Na}} \mathrm{V}$. When distal $\mathrm{Na}$ supply is augmented at low levels of solute clearance in salt-free subjects, $\mathrm{T}^{\mathrm{c}} \mathrm{H}_{2} \mathrm{O}$ increases to levels obtained in high salt studies. The lower $\mathrm{T}^{\mathrm{c}} \mathrm{H}_{2} \mathrm{O}$ levels obtained in salt-free subjects may therefore result from diminished distal $\mathrm{Na}$ supply (secondary to enhanced proximal $\mathrm{Na}$ reabsorption or a decreased GFR, or both). Increasing distal $\mathrm{Na}$ supply at high levels of solute clearance dose not augment $\mathrm{T}^{\mathrm{c}} \mathrm{H}_{2} \mathrm{O}$-conceivably because a solute diuresis provokes an increase in effective medullary blood flow.

Insulin-like Activity of Extracts from Large Sarcomatous Tumors Associated with Hypoglycemia. JURGEN Steinke, J. Stuart Soeldner and Albert E. RenOLD,* Boston, Mass.

Association of large mesothelial tumors with severe hypoglycemia is a recognized clinical syndrome which can be corrected by surgical removal of the tumor. The mechanism of the hypoglycemia is poorly understood. Large samples from five such tumors (all identified as sarcomatous), weighing between 1,070 and $7,500 \mathrm{~g}$, have been examined for insulin-like activity. Each sample was 1) homogenized in buffer or water, centrifuged, and the supernatant assayed, and 2) extracted with acidethanol according to Scott and Fisher, followed by dialysis, lyophylization, and assay. The assay procedure employed was that using the oxidation of glucose-1- $\mathrm{C}^{\mathbf{1 4}}$ by rat adipose tissue in vitro as an index of insulin activity. Direct assay of the homogenate supernatant revealed levels of insulin-like activity ranging from 0.002 to $0.005 \mathrm{U}$ per $\mathrm{g}$ of tumor (4.3 to $16.2 \mathrm{U}$ per total tumor). The activity of the acid-ethanol extracts ranged from 0.0001 to $0.006 \mathrm{U}$ per $\mathrm{g}$ ( 0.2 to $6.4 \mathrm{U}$ per total tumor). The values obtained after acid-ethanol extraction are within the range found for similar extracts of samples from human liver, kidney, adrenal, and lymph nodes, and are probably due to contamination with blood insulin-like activity. By way of comparison, extracts of normal pancreas assayed by the same technique exhibited 1.5 to $3.0 \mathrm{U}$ per $\mathrm{g}$, and seven pancreatic islet cell tumors associated with hypoglycemia exhibited 2.7 to $28.5 \mathrm{U}$ per g. These findings on sarcomas with hypoglycemia contrast with the elevated insulin-like activity of a tumor extract assayed in this laboratory (as reported by August and Hiatt) and also with isolated reports in the literature. It is concluded that hypoglycemia associated with large mesothelial tumors is not necessarily related to the presence of significant amounts of extractable insulin-like material in the tumor.

The Role of the Phenolic Hydroxyl Group of Thyroxine in Binding by Human Serum Albumin. KenNeTH Sterling* and Thornton S. Walker, New York, N. Y.

Previous equilibrium dialysis studies have suggested that the binding of thyroxine by human serum albumin requires interaction between the dissociated (anionic) phenolic hydroxyl group of thyroxine and cationic groups on the protein molecule, probably epsilon-amino groups of lysine residues. To test the importance of the dissociable hydroxyl (phenolate) group of thyroxine in the interaction, studies have been carried out with methoxythyroxine, the methyl ether of thyroxine, which cannot ionize at this site. Addition of "cold" methoxy-thyroxine to human serum albumin had no detectable effect upon the binding of $\mathrm{I}^{131}$-labeled thyroxine in the equilibrium dialysis system, when 1,2 , or 3 moles of the methoxy compound were added per mole of thyroxine. Since comparable thyroxine additions reduced the fraction bound, it is evident that the phenolate group is required at this concentration range. However, the addition of 4 or 6 moles of methoxy-thyroxine per mole of thyroxine resulted in a slight but definite diminution of $\mathrm{I}^{131}$ thyroxine bound to albumin. Since large amounts of methoxy-thyroxine were needed to demonstrate displacement, the findings suggest that the anionic phenolate group of thyroxine plays a facilitating role in binding by human serum albumin.

Retardation of Sodium Flux in"Dog Erythrocytes by Physiological Concentrations of Aldosterone In Vitro. David H. P. Streeten and Colette Spach, Syracuse, N. Y. (introduced by Eugene L. Lozner).

Previous attempts to demonstrate effects of physiological concentrations of aldosterone on sodium exchange in isolated tissues have been unsuccessful. The possibility that such effects might be demonstrable in dog erythrocytes (which have unusually high sodium concentrations) before and after adrenalectomy, was investigated. Samples of blood from a dog were incubated in stoppered beakers to which $\mathrm{Na}^{22} \mathrm{Cl}$ and dextrose were added, and the mixture was agitated for 2 hours in an atmosphere of $95 \%$ oxygen and $5 \% \quad \mathrm{CO}_{2}$. Aldosterone was added in varying concentrations to the blood in three of four beakers, the fourth serving as control. Aliquots of blood were removed from the beakers at 15-minute intervals for determinations of plasma radioactivity, stable $\mathrm{Na}$ concentration, and hematocrit. From 
the calculated regression line representing the rate of disappearance of radioactivity from the plasma, $\mathrm{Na}$ flux rates were determined. Before adrenalectomy, a significant $(\mathrm{p}<0.01)$ inverse dose-response relationship was found between $\mathrm{D}$-aldosterone concentrations of from 30 to $114 \mu \mathrm{g}$ per $\mathrm{L}$ and $\mathrm{Na}$ flux rates. These concentrations are still considerably above physiological levels. Blood from the same dog after bilateral adrenalectomy, during maintenance therapy with cortisol and saline only, disclosed a highly significant $(p<0.001)$ inverse dose-response relationship between $\mathrm{D}$-aldosterone within the physiological range of concentration $(0.2$ to $2.0 \mu \mathrm{g} / \mathrm{L})$ and $\mathrm{Na}$ flux rates. Above $2.0 \mu \mathrm{g}$ D-aldosterone per $\mathrm{L}$ the dose-response relationship no longer obtained, and $\mathrm{Na}$ flux was reduced maximally to about one-fifth control rates. In the absence of added aldosterone, the mean $\mathrm{Na}$ flux was significantly $(p<0.001)$ higher in blood drawn after adrenalectomy (10.4 mEq/L of cells/ hr) than before the operation $(6.5 \mathrm{mEq} / \mathrm{L}$ of cells $/ \mathrm{hr})$. It is concluded that physiological concentrations of aldosterone have striking inhibitory effects on the movement of sodium into the erythrocytes of the dog.

Interconversion of Acanthocytes and Normal Erythrocytes with Detergents. SAm Switzer and Howard A. EDER,* New York, N. Y.

Acanthocytosis (Bassen-Kornzweig syndrome) is a familial disorder characterized by retinitis pigmentosa, progressive ataxia, steatorrhea, absence of low density lipoproteins, and malformed erythrocytes. These cells, known as acanthocytes, are spherical with numerous projecting spines, and are diagnostic of this disease. We have observed that acanthocytes ( $1 \%$ suspension) exposed in vitro to a nonionic detergent, Tween $80\left(10^{-4} \mathrm{M}\right)$, are rapidly converted to the biconcave disc shape of normal erythrocytes. Nonionic detergents contain a hydrophilic portion (usually a polyoxyethylene polymer of variable molecular weight, or a polyol, or both) in ester or ether linkage with a lipophilic portion which is frequently a fatty acid derivative. In a series of compounds studied it was found that the hydrophilic portion does not influence acanthocyte reversal, but specificity was demonstrated for the lipophilic portion; oleyl derivatives convert acanthocytes to normal, whereas, stearyl derivatives do not. There is evidence for competition between oleyl and stearyl derivatives, and studies with $\mathrm{C}^{14}$-Tween 60 (stearyl) indicate binding of the detergent by erythrocytes. No relation could be demonstrated between the surfactant properties of the nonionic detergents and their ability to convert acanthocytes to normal. Ionic detergents do not reverse acanthocytes. Many such agents, however, produce in normal erythrocytes a sequence of morphologic changes described by Ponder as the "disc-sphere transformation." Intermediate forms seen in this sequence are indistinguishable from acanthocytes. Furthermore, many agents unrelated to nonionic detergents but known to reverse the discsphere transformation convert acanthocytes to normal shape and, conversely, nonionic detergents, which con- vert acanthocytes to normal, reverse the disc-sphere transformation.

The Effect of Renal Perfusion Pressure on the Active Transport of Sodium out of Distal Tubular Urine as Studied with the "Stop-Flow" Technique. LouIs Tobian,* Karen Coffee, Dorothy Ferreira and Judith Meuli, Minneapolis, Minn.

Kidneys perfused at $170 \mathrm{~mm} \mathrm{Hg}$ excrete considerably more sodium than those perfused at $100 \mathrm{~mm} \mathrm{Hg}$. "Stopflow" experiments helped to explain why. "Normal" rats without kidneys or adrenals supplied arterial blood through a pump to isolated normal kidneys and simultaneously received renal venous blood and urine. In 13 experiments the pump perfused the isolated kidney at $170 \mathrm{~mm} \mathrm{Hg}$ and, in 10 other experiments, at 100 . In all experiments the ureter was clamped after 60 minutes of perfusion. Ureteral pressures "plateaued" within the next 4 minutes, after which clamping was continued for 8 minutes. Then the ureter was unclamped and urine collected in polyethylene tubing. Sodium concentration was determined in successive segments of the tubing. Each "stop-flow" collection showed the typical smooth drop in sodium concentration. The segment with the lowest sodium concentration represents the maximum ability of that kidney to pump sodium out of the distal tubular urine. The lowest sodium concentrations averaged $31.3 \mathrm{mEq}$ per $\mathrm{L}$ in the 13 experiments with perfusion at 170 ; the lowest concentrations averaged 14.2 in the 10 experiments with perfusion at $100(\mathrm{p}<$ 0.000006 ). Thus, kidneys perfused at $100 \mathrm{~mm} \mathrm{Hg}$ pump sodium more avidly. The effect is not related to aldosterone since adrenal tissue was absent. It is not related to different rates of glomerular filtration because clamping largely stops glomerular filtration. Medullary blood flow is undoubtedly greater at the higher perfusion pressure; but under "stopped" conditions, this should not prevent distal tubular cells from pumping sodium maximally. The best explanation is that humoral substances are secreted within the kidney itself, in greater or lesser amounts, depending upon the distention of the renal arterial system. These substances directly control the rate at which the distal tubular cells pump sodium out of the tubular urine. This mechanism may play an important part in various sodium-retaining syndromes.

Methotrexate-induced Intestinal Epithelial Damage Demonstrated by Electron Microscopy but not Revealed by Light Microscopy. Jerry S. Trier, Bethesda, Md. and Seattle, Wash. (introduced by Cyrus E. Rubin).

After administration of 2 to $5 \mathrm{mg}$ i.v. of the folic acid antagonist, methotrexate, per $\mathrm{kg}$, light microscopy of peroral biopsies of human jejunal mucosa revealed no changes in the villi although there were some crypt alterations. The crypts exhibited almost complete mitotic inhibition for 48 hours or more and intracytoplasmic DNA-positive bodies in some crypt cells. This paper describes the marked fine structural changes which were 
clearly revealed by electron microscopy, although they were unapparent by light microscopy. Serial jejunal biopsies in six patients at various intervals after methotrexate administration showed striking fine structural alterations in both crypt and villus which appeared within 6 hours, lasted for at least 48 hours, and reverted to normal within 96 hours. These structural changes involved absorptive, crypt, goblet, and Paneth cells although many cells of each type were completely spared. Damaged cells showed swollen, fragmented mitochondria and strikingly dilated cysternae of the Golgi material and endoplasmic reticulum. Altered microvilli were seen in some absorptive cells. Some of the crypt cells showed clumping of the nucleoplasm or intracytoplasmic inclusions whose fine structure suggested cellular remnants, or both. Despite these extensive fine structural changes, the histologic architecture of the villi was unaltered and the patients noted minimal or no gastrointestinal symptoms. After methotrexate, severely damaged absorptive, crypt, and Paneth cells were seen adjacent to morphologically normal ones. This cell sparing indicates a marked variation in sensitivity to methotrexate among individual cells within a cell population. These doses of methotrexate arrest small intestinal cell replacement temporarily and produce profound alterations in fine structure. These changes could easily be missed clinically and by ordinary light microscopy.

Newer Lipids of Human Serum, Cerebrospinal Fluid, and Tissues. NaIP Tuna, Helmut K. Mangold, Rudolph Kamerex and Mary L. Louden, Minneapolis and Austin, Minn. (introduced by Ivan D. Frantz, Jr.).

Human serum, cerebrospinal fluid, aortic atheromatous plaques, liver, kidney, spleen, perinephric fat, and bone marrow were extracted with 2:1 chloroform-methanol (vol/vol), according to Folch, and the total lipid extracts were analyzed by a combination of silicic acid column and thin layer chromatography. In every lipid analyzed, in addition to the well known major lipid classes (phospholipids, cholesterol, free fatty acids, triglycerides, and cholesterol esters), the following lipid compounds, present in smaller concentrations, were separated and identified by gas-liquid chromatography, thin layer silicic acid chromatography, infrared spectroscopy, and identification of their oxidation and reduction products. 1) Trimeres (trioxanes) have not, heretofore, been reported as occurring in animal or human tissues. They represent polymerized, long chain aldehydes, are less polar than cholesterol esters, yield dimethyl acetals with hydrochloric acid-methanol, and have $\mathrm{Rf}$ values similar to those of "branched" fatty acid methyl esters when separated by gas-liquid chromatography. 2) Fatty acid methyl esters result, we believe, from the methylation of free fatty acids during extraction with chloroformmethanol, since they were not encountered when the extraction was carried out with chloroform-ethanol (2:1, vol/vol). 3) Glyceryl ether diesters (alkoxydiglycerides) are less polar than triglycerides, and upon reduc- tion with lithium aluminum hydride yield glyceryl ethers which were separated by thin layer chromatography. 4) Aldehydogenic triglycerides have not as yet been separated in pure form. Evidence will be presented for their existence. 5) Several sterols other than cholesterol were separated but not identified. The biological significance of these substances will be discussed.

Effect of a Single and Repeated Dose of "Pancreozymin" on Pancreatic Exocrine Secretion. Malcolm P. Tyor, David A. Giordano and Edward E. Owen, Durham, N. C. (introduced by Julian M. Ruffin).

Considerable debate has centered about the uniformity of pancreatic enzymatic response to various stimuli. The present report deals with such measurements obtained from anesthetized dogs after administration of "pancreozymin" (Vitrum). Pancreatic flow was maintained by constant infusion of secretin, 0.88 to $1.08 \mathrm{U}$ per minute, in ten dogs. Secretions were collected from the cannulated pancreatic duct for 15-minute periods, beginning 45 minutes after completion of surgery (bile duct cannulation, gastrostomy, and pyloric ligation) and onset of secretin administration. In each instance, $20 \mathrm{U}$ of pancreozymin was given intravenously after the eighth collection period. Six dogs were given a second identical dose 2.75 hours later. Each 15-minute sample was analyzed for protein concentration and amylase and lipase activities. Monoesters of $\beta$-napthyl actetate, caprylate, and laurate were used as substrate for lipase activity; solubilization of the latter two was obtained using sodium taurocholate. The pattern of response to the initial dose of hormone was similar in all dogs, i.e., an abrupt increase in volume (1.8-fold), protein content (13-fold), amylase activity (13-fold), and esterase or lipase activity: acetate (29-fold), caprylate (25-fold), laurate (27-fold). A fall to baseline was observed at the 45minute post-hormone period. Thereafter, mean values for all measurements were significantly lower than those in the prehormone periods, despite a significant increase in volume. The immediate response to the second dose of pancreozymin was similar. These data confirm previous observations on the magnitude of the enzymatic response to pancreozymin and characterize the posthormonal effect. Although the enzymatic response was temporally uniform, the data suggest that pancreozymin may enhance lipase activity as compared with amylase, since the magnitude of the response to pancreozymin was similar whether or not taurocholate was employed in the quantitation of esterase activity.

Metabolic Changes and Viability of Stored Human
Platelets. P. UATHAviKUL AND M. BaLdini, Boston, Mass. (introduced by William Dameshek).

Previous investigations in this laboratory have shown that storage of blood platelets at $4^{\circ} \mathrm{C}$ produces rapid loss of their "viability," i.e., their capacity to recirculate and survive after infusion. Because of the known high rate of platelet glycolytic metabolism, the idea was 
formulated that interruption of regeneration of energyrich phosphate compounds in the stored platelets might be totally or mainly responsible for their loss of viability. To this end, the rate of disappearance of the "7-minute phosphorus" ( 7 min-P), representing mainly ATP, in human platelets preserved in various media at $4^{\circ} \mathrm{C}$ was determined by differential hydrolysis. Enzymes of glycolysis (PHADH, PGK, hexokinase) were also measured. Correlation of these values with the rate of decrease in viability of platelets preserved in these same media was studied. Viability was measured in vivo by the $\mathrm{Cr}^{51}$-labeling technique. The 7 min-P decreased rapidly in platelets preserved in saline medium. After 5 days of storage, only $24 \%$ of the original value was obtained. The use of plasma medium improved preservation of the $7 \mathrm{~min}-\mathrm{P}$. Addition of adenosine to the plasma had favorable effect. Addition of inosine plus adenine had the greatest effect, with $80 \%$ of the original 7 min-P found after 14 days of storage. The rate of decrease in the activities of the enzymes tested grossly paralleled the rate of disappearance of the $7 \mathrm{~min}-\mathrm{P}$. Storage media which favorably affected preservation of the $7 \mathrm{~min}-\mathrm{P}$ also had favorable effect on preservation of viability. However, with all the media studied, the rate of decrease in viability was strikingly more rapid than the rate at which the 7 min-P was lost. This indicated that decrease of ATP in platelets during storage was not the only and probably not the main metabolic damage which determined loss in viability.

The Antibody Response to Bacteriophage $\phi X 174$ in Nezuborn Premature Infants. J. W. Uhr,* J. DANCIS, E. C. Franklin,* M. S. Finkelstein and E. W. LEwIS, New York, N. Y.

Premature newborn infants and older children show a similar antibody response to injection of minute amounts of bacteriophage $\phi \mathrm{X} 174$ as judged by the following criteria: 1) easily detectable neutralizing antibody is produced during the first week; 2) the titer rises for an additional 2 to 3 weeks; 3 ) an efficient secondary antibody response can be achieved by reimmunization 6 weeks later; 4) a change occurs in the molecular species of antibody, from $19 \mathrm{~S}$ to $7 \mathrm{~S}$ sedimentation constant 2 to 6 weeks after immunization. These observations contrast with past studies demonstrating newborn incompetency toward other antigens, and suggest that immunologic maturation may occur in two or more steps.

The Secretion of 18-Dihydroaldosterone by the Human Adrenal. Stanley Ulick and Kathry Kusch Vetter, New York, N. Y. (introduced by Solomon A. Berson).

A study of mineral hormone biosynthesis, using the bullfrog adrenal as a simple model of the zona glomerulosa of the mammalian adrenal cortex, led to the isolation of a new steroid in the aldosterone series. On the basis of chemical degradative studies and conversion to a known steroid, lactone, the structure 18-dihydroaldosterone (18-hydroxycorticosterone) was assigned. Evidence for the production of the new steroid by the human adrenal was obtained when the previously described urinary metabolite of aldosterone was separated into two tetrahydro derivatives, $3 \alpha, 18,21$-trihydroxy-11,20diketopregnane (I), and $3 \alpha$-hydroxy-5 $\beta$-tetrahydroaldosterone (II). When aldosterone- $\mathrm{H}^{3}$ and the $\mathrm{C}^{14}$-labeled 18-dihydro derivative were injected simultaneously and both urinary products isolated, II was found to be a metabolite of aldosterone and I a metabolite of 18dihydroaldosterone. Labeled 18-dihydroaldosterone was injected and the rate of production of the steroid estimated from the dilution in specific activity of urinary metabolite I. Normal individuals secreted larger amounts of the 18-dihydro derivative than of aldosterone as did patients with secondary hyperaldosteronism in whom secretion of the 18-dihydro derivative reached values of $4.0 \mathrm{mg}$ per day. 18-Dihydroaldosterone exhibited low activity as a mineral hormone (courtesy of Dr. J. G. Llauredo). Its secretion appeared to reflect the activity of the renal pressor system, as was illustrated by a study of malignant hypertension associated with unilateral renal disease. The secretion of aldosterone was 600 and $800 \mu \mathrm{g}$ per day and of the 18-dihydro derivative, 2,900 and $3,400 \mu \mathrm{g}$ per day. Removal of the diseased kidney led to clinical improvement and a decrease in the secretion of both steroids (aldosterone: 150, $155 \mu \mathrm{g} / \mathrm{day}$; 18-dihydroaldosterone: $1,600,1,800 \mu \mathrm{g} /$ day).

The Role of Endogenous Glucagon in Blood Glucose Regulation in Man. ROgER H. UNGER AND ANNA M. Eisentraut, Dallas, Tex. (introduced by Elias Strauss).

Inability to measure circulating endogenous human glucagon has prevented clarification of its hormonal status in man. Modifications of a previously described specific radioimmunochemical glucagon assay to permit the recovery from serum of as little as 100 to $250 \mu \mu \mathrm{g}$ per $\mathrm{ml}$ of added crude human glucagon made possible the following studies, designed to examine the role of glucagon in blood glucose homeostasis by determining the effects of starvation and hypoglycemia upon its serum concentration. The fasting serum glucagon level in normal humans averaged $100 \mu \mu \mathrm{g}$ per $\mathrm{ml}(0$ to 400$)$. During hypoglycemia induced by 3 -hour infusions of glucagon-free (immunochemically established) insulin (0.1 $\mathrm{U}$ per $\mathrm{kg}$ ), rises in serum glucagon of 250 and $350 \mu \mu \mathrm{g}$ per $\mathrm{ml}$ appeared in 2 of 3 subjects after an hour; modest reduction of glucagon followed glucose loading. Six normals and one mild diabetic were fasted for 72 hours; after 48 hours of starvation, all seven exhibited striking increases in serum glucagon from a mean of 112 (0 to $400)$ to $326 \mu \mu \mathrm{g}$ per $\mathrm{ml}(160$ to 425$)$ and at 72 hours to $663 \mu \mu \mathrm{g}$ per $\mathrm{ml}(500$ to 800$)(\mathrm{p}<0.01)$; FFA increased by 530 to $1,960 \mu \mathrm{Eq}$ per $\mathrm{L}$, while blood glucose declined an average of $25 \mathrm{mg}$ per $100 \mathrm{ml}$ and body weight 
from 2.8 to $8.2 \mathrm{~kg}$. Glucose loading produced within 3 hours moderate but significant suppression $(p<0.01)$ of serum glucagon to a mean of $488 \mu \mu \mathrm{gEq}$ per $\mathrm{ml}$ (375 to 600), and FFA declined; glucose tolerance was impaired in all subjects. In summary, immunochemical measurements of serum glucagon in man reveal: 1) a rise in glucagon during starvation and, less consistently, during hypoglycemia; 2) partial suppression of this rise in glucagon during glucose loading; 3) essentially parallel fluctuations in glucagon and FFA. Glucagon appears to be a true human hormone with a role in blood glucose regulation, possibly that of maintaining glucose distribution to glucose-dependent tissues during glucose deprivation. The possibility is suggested that the hyperglucagonemia of starvation may contribute to the impaired glucose tolerance of "starvation diabetes" by limiting the decline in hepatic glucose output which normally follows glucose loading.

Effects of Humoral Factors on Antigenic Histamine Relcased from Human Leukocytes. PAUL P. VAN ArSdel, JR., Seattle, Wash. (introduced by Clement A. Finch).

When leukocytes from a person with atopic sensitivity are incubated with specific antigen, free histamine is released from these cells in proportion to the logarithm of antigen concentration up to an optimal point. Excess antigen releases less histamine. This phenomenon has made possible several quantitative, experimental observations on the nature of human skin-sensitizing antibody. For the present study, heparinized blood from subjects sensitive to grass pollen was collected and used within 30 minutes. In all instances, suspensions of thricewashed leukocytes were prepared and incubated for 1 hour with water-soluble pollen antigen at a concentration of $0.01 \mu \mathrm{g}$ pollen nitrogen per ml. After centrifugation, histamine in each supernatant solution was measured photofluorimetrically. Two aliquots of leukocytes from each of eight sensitive donors were suspended in Tyrode's solution, and two others in isologous plasma. The amount of histamine released by antigen varied from 9.5 to $44 \mu \mathrm{g}$ per L. Little variation was noted on repeat studies of any one patient. Unrelated antigens released no histamine, nor was histamine released from normal donor cells by antigen. These eight donors had received no parenteral immunization with pollen antigen. The presence of plasma did not alter the amount of histamine released even though each plasma contained a high titer of skin-sensitizing antibody. The effect of plasma from patients who had been artificially immunized by repeated injections of the same antigen was quite different. Antigenic histamine release from their leukocytes suspended in Tyrode's solution was in the same range as that of the untreated donors. However, almost no histamine release occurred in the presence of isologous plasma, unless higher antigen concentration was used $(0.1 \mu \mathrm{g}$ pollen nitrogen $/ \mathrm{ml})$. Serum from two of the artificially immunized donors has been fractionated by diethylaminoethyl cellulose chromatography. The two fractions containing $\boldsymbol{\gamma}_{1^{-}}$and $\boldsymbol{\gamma}_{2}$-globulins inhibited histamine release in a manner comparable to that of whole plasma. Another fraction, containing 18S $\gamma$-globulin, was partially inhibitory. Two other protein fractions had no effect. These observations suggest that spontaneous atopic sensitivity is a cellular phenomenon, not directly mediated by free skin-sensitizing antibody. Contrariwise, the effects of artificial immunization are primarily humoral, associated with the production of several $\gamma$-globulins, and perhaps other proteins, capable of neutralizing antigen.

Practical Growth Hormone from Human Pituitary Glands. W. P. VanderLaAn,* U. J. Lewis ANd A. E. Jones, La Jolla, Calif.

A simple procedure for preparing human growth hormone, in which hot glacial acetic acid extraction of acetone-dried powder was followed by precipitation with acetone and lyophilization, gave a 10 to $12 \%$ yield. The material was dissolved in dilute $\mathrm{HCl}$ and administered intramuscularly in clinical use. Assay showed no thyrotropic or gonadotropic activity. Despite failure to remove corticotropin, the material showed greater homogeneity than the Raben preparation when tested by disc electrophoresis on acrylamide gel. Clinically the material sustained over a 3-month period the rate of growth and elevated alkaline phosphatase activity of an 18 year old dwarf who has now grown a total of 9 inches in 38 months. 17-Hydroxycorticosteroid excretion remained low despite $2 \mathrm{mg}$ intramuscularly on alternate days, and in the 24 hours following $5 \mathrm{mg}$ intramuscularly no rise in corticosteroid excretion was measurable. After overnight fasting, free fatty acids in two pituitary dwarfs rose 4 hours after $5 \mathrm{mg}$ of the material was given intramuscularly (338 to $920 \mu \mathrm{Eq} / \mathrm{L} ; 665$ to $968 \mu \mathrm{Eq} / \mathrm{L}$ ). Avoidance of alkali in the preparation accounts for the greater homogeneity. Lack of corticotropic effect appears to depend on the low initial corticotropin content of human pituitaries and the inconsequential "flash" effect when it is given in aqueous preparation intramuscularly. The preparation, therefore, is simple and suitable for human use.

Factors of Rate and Kinetics of Cellular Reduplication in Human Hair Roots. Eugene J. VAN ScotT AND Thomas M. Ekel, Bethesda, Md. (introduced by $\mathrm{C}$. Gordon Zubrod).

The rapid rate of hair growth suggests that the germinative matrix of the hair manifests a rate of cellular reduplication equal to or exceeding that of any tissue of the body. The size of the germinative matrix of human scalp hairs compared to the size of hair shaft produced daily indicates that the matrix reduplicates its cellular population once every 24 hours. Some factors possibly relating to the control of this benign orderly growth were studied. Ninety-two hair roots, from ten biopsy specimens of normal human scalp, were histo- 
geometrically examined in serial sections $6 \mu$ thick. The volumes of the entire germinative matrix and constituent $30-\mu$ thick segments were determined. The number of cells in mitosis in each segment was determined and the mitotic index calculated. The volume and cell population of each connective tissue hair papilla were determined. Distances between component portions of the hair root were measured. The total number of mitoses in the hair matrix was found to be proportional to the volume of the matrix. A ratio of approximately eight connective tissue papilla cells per one mitosis in the matrix was found in hair roots of different sizes. The mitotic index of the hair matrix was constant for hairs of different sizes, and comparable segments had equal mitotic indices. Mitotic index was highest in the most proximal segment, 84 per 1,000 cells $\left(10^{6} \mu^{3}\right)$, and lowest in the most distal segment, with a value of 2 . The mitotic index of any segment of any sized hair root was found to be expressed by the formula $X=$ $113-0.58 Y$, where $Y$ is the distance in microns of the segment from a midpoint in the base of the hair papilla.

Relation of Fecal Flora to Endogenous Infections of the Urinary Tract. Kenneth L. Vosti, ARnold S. Monto And Lowell A. Rantz,* Palo Alto, Calif.

The serologic classification of Escherichia coli according to specific $O$ groups has afforded an opportunity to further our understanding of the pathogenesis of infections of the urinary tract. These studies represent analyses of urine, stool, and blood specimens from 80 patients with urinary tract infections, 24 of whom had bacteremia. Stool specimens often contained multiple serologic groups; only a single group was usually identified in urine and blood. Thirty-four different groups were identified in stool, 17 in urine, and 8 in blood specimens. The same serologic groups were found in paired stool and urine specimens from $75 \%$ of 62 patients with infections of the urinary tract, in paired urine and blood specimens from 12 of 13 patients with bacteremia, and from 6 of 7 with paired stool and blood specimens. In 5 of 6 patients the same $O$ groups were present in blood, urine, and stool specimens. Groups $1,4,6,15,25$, and 75 comprised $18 \%$ of the total number of different groups identified in stool, $35 \%$ in urine, and $50 \%$ in blood specimens. These groups represented $43 \%$ of the total number of groups identified in the individual stool and $70 \%$ of those in the individual urine and blood specimens. Groups 4 and 6 accounted for 20,37, and $59 \%$ of these values, respectively. These studies show that: 1) individual urine and blood specimens usually contain $E$. coli of a single serologic group in contrast with stool specimens where multiple groups may be found; 2) most of the patients with urinary tract infections have the same serologic group in paired urine and stool specimens, and in the blood when bacteremia occurs; and 3) a few groups are more commonly associated with infections of the urinary tract and bacteremia.
Dextran Gel Filtration of Serum Proteins Involved in Host Defenses Against Infection. SHeLdon L. WAGNER and Gene H. Stollerman,* Chicago, Ill.

The molecular sieving effects of highly cross-linked dextran gels (Sephadex) were applied to the purification of serum proteins involved in host defenses against infection. In particular, the rapid removal of antibiotics and of other antibacterial substances (various preservatives) from serum was attempted in order to aid in the study of acute-phase serological responses in patients with fever. Two- to 3-ml serum samples were filtered at $4^{\circ} \mathrm{C}$ through G-75 Sephadex columns. This procedure separated the effluent samples completely into two fractions, greater and lesser than molecular weight of 40,000 , respectively. Serum proteins, which did not diffuse into the gel strains, moved rapidly through the column with minimal dispersion and were recovered within 1 hour in buffers of desired $\mathrm{pH}$ and ionic strength. The total serum protein was diluted less than $20 \%$. Peak protein concentrations in individual effluent samples almost equaled those of the native sera. The effluent fractions of serum proteins, collected in Tyrode's buffer, were compared with native serum for the following biological activities: 1) complement, 2) cofactors in human serum required for efficient opsonization of group A streptococci, 3) "gram-positive" bactericidins, and 4) "gram-negative" bactericidins. All activities were recovered fully with minimal dilution. Human sera containing penicillin, erythromycin, chloramphenicol, tetracycline, or streptomycin, in various combinations, were subjected to gel filtration. By assays against selected susceptible strains of bacteria, the gel-filtered serum proteins were found to be free of antibiotic activity. The antibiotics were recovered, free of serum protein, in the second, smaller molecular weight fraction. The method provides a useful initial step in the purification of serum proteins associated with antibacterial activity, and simplifies the study of host defense factors in the blood of patients receiving chemotherapy for infections.

Aldosterone and Progesterone Secretion in Pregnancy. Mamoru Watanabe, Oscar V. Dominguez, C. Irving Meeker, Ethan A. H. Sims, Mary Jane Gray and Samuel Solomon, Montreal, Canada and Burlington, Vt. (introduced by John C. Beck).

Aldosterone and progesterone secretion rates ( $A_{\mathrm{si}}$ and $\mathrm{P}_{\mathrm{sr}}$ ) were determined in the third trimester of pregnancy with isotope dilution techniques using $\mathrm{H}^{8}$-labeled hormones. $\mathrm{A}_{\mathbf{s r}}$ were measured in 59 normal pregnant subjects between 27 and 40 weeks of gestation who were on known sodium intakes of 50 to $225 \mathrm{mEq}$ per day. The range was 307 to $2,912 \mu \mathrm{g}$ per day (normal nonpregnant subjects, 88 to $216 \mu \mathrm{g} /$ day) with a mean value of $1,127 \mu \mathrm{g}$ per day. Seventy $\%$ of the $A_{\mathrm{sr}}$ were between 500 and $1,300 \mu \mathrm{g}$ per day. $\mathrm{Na}$ restriction markedly elevated the $A_{s r}$ (to $10,000 \mu \mathrm{g} /$ day) whereas $\mathrm{Na}$ loading usually depressed it. In 52 patients on a normal $\mathrm{Na}$ intake (120 to $190 \mathrm{mEq} /$ day) there was a uniform rate of 
secretion between 30 and 35 weeks of gestation with a rise thereafter. In severe pre-eclamptics the $A_{\mathbf{s r}}$ was found to be in the normal nonpregnant range, whereas in mild pre-eclamptics the secretion rate was normal or slightly elevated. In 50 normal subjects in the third trimester of pregnancy the $P_{\text {sr }}$ had a range of 188 to 563 $\mathrm{mg}$ per day with a mean value of $329 \mathrm{mg}$ per day. When simultaneous $A_{\mathbf{s r}}$ and $P_{\mathbf{s r}}$ were determined, no direct correlation between the two secretion rates could be demonstrated. With $\mathrm{Na}$ restriction the $\mathrm{A}_{\mathrm{Br}}$ was markedly elevated without much change in the $\mathrm{P}_{\mathbf{s r}}$. Intrauterine fetal death invariably resulted in a fall of the $A_{s r}$ to nonpregnant levels, whereas the $P_{s r}$ was not always decreased. The above findings are in harmony with the view that the marked stimulation of aldosterone secretion observed in late pregnancy, especially with $\mathrm{Na}$ restriction, is due in part to the necessity for counteracting the large amounts of progesterone produced, and in part in response to the need for maintaining a positive sodium balance previously described.

Relationship of High Levels of "Bart's" Hemoglobin in Infancy to $\alpha$-Thalassemia. DAvid J. Weatherall, Baltimore, Md. (introduced by C. Lockard Conley).

Hemoglobin patterns of umbilical cord blood from 826 Negro and 150 Caucasian infants were studied by electrophoresis on vertical starch gel. Sixteen Negro infants showed 5 to $10 \%$ of a rapidly migrating hemoglobin which chemical studies, including "fingerprinting," identified as hemoglobin "Bart's" $\left(\boldsymbol{\gamma}_{*}^{\mathrm{F}}\right)$. Ten of these infants had repeated hematologic studies up to the age of 1 year. Bart's hemoglobin disappeared at about 6 months, but striking abnormalities of the red cells and decreased osmotic fragility persisted. One parent of each infant and 11 of 28 siblings showed similar although milder abnormalities of the erythrocytes. Traces of a hemoglobin with the mobility of hemoglobin $\mathrm{H}$ were demonstrated in nine of these apparently affected family members, but in no instance was the level of fetal hemoglobin or of hemoglobin $A_{2}$ increased. Three siblings who were heterozygous for hemoglobin $\mathrm{S}$ or $\mathrm{C}$ had more hemoglobin A than abnormal hemoglobin, but they displayed erythrocyte abnormalities exceeding those usually seen in S-trait or C-trait and were presumably doubly affected. Many normal newborn infants, both Negro and Caucasian, showed traces of a hemoglobin with an electrophoretic mobility identical to that of hemoglobin Bart's. Increased levels of Bart's hemoglobin in infancy may result from an inherited defect in $\alpha$-chain synthesis ( $\alpha$-thalassemia). During rapid synthesis of hemoglobin in fetal life this defect leads to an excess of $\gamma^{\mathbb{F}}$ chains, resulting in the production of hemoglobin Bart's and traces of hemoglobin $\mathrm{H}\left(\beta_{4}^{\mathbf{\Delta}}\right)$. This deficiency of $\alpha$-chains is further exaggerated during the switchover from fetal to adult hemoglobin synthesis, since both $\gamma$ - and $\beta$-chains are competing for available $\alpha$-chains, a fact which may explain the small amounts of Bart's hemoglobin found in many normal infants.
In adult life this form of thalassemia is manifested by very mild hematologic abnormalities.

New Approach to the Clinical Pharmacology of Digitalis in Man. Arnold M. Weissler, Harvey E. Grode and William G. Gamel, Columbus, Ohio and Galveston, Tex. (introduced by James V. Warren).

Previous studies in normal individuals demonstrating a decrease in the duration of mechanical systole after administration of deslanoside (Cedilanid-D) have suggested the application of this physiologic effect in the assay of digitalis effect in man. In the present investigation this technique was employed in the study of the duration of deslanoside effect and the dose-response relationships in normal individuals. Serial determinations of the duration of mechanical systole, as derived from simultaneous recordings of the first to second interval $\left(\mathrm{S}_{1}-\mathrm{S}_{2}\right)$ on a phonocardiogram and the duration of left ventricular ejection (ET) on the carotid arterial pulse tracing, were made for 5 days in nine normal individuals after administration of deslanoside, $1.6 \mathrm{mg}$ i.v. The digitalis effects were compared to 4 control days of observation. Maximum decrease in $\mathrm{S}_{1}-\mathrm{S}_{2}$ and ET (corrected for heart rate) averaged, respectively, 21 $(p<0.001)$ and $18 \mathrm{msec}$ per beat $(\mathrm{p}<0.001)$ and appeared 1 to 2 hours after drug administration. Significant shortening in the duration of mechanical systole continued until 72 hours after administration of the drug. Comparison of the effects of 1.6 and $0.8 \mathrm{mg}$ i.v. in the same individual during the first 24 hours after drug administration was made in seven subjects. A parallel temporal effect of the two dose levels was observed. The degree of shortening in $\mathrm{S}_{1}-\mathrm{S}_{2}$ and ET after the 0.8 $\mathrm{mg}$ dose was $66 \%$ and $84 \%$ of that of the $1.6 \mathrm{mg}$ level. These observations demonstrate the applicability of these techniques in the clinical assay of digitalis effect, and suggest the need for re-evaluation of our concept of the dose-response relationship of deslanoside in the human.

The Metabolism of Certain Isotopically Labeled Pyrimidines and the Rate of Pyrimidine Biosynthesis in Man. S. M. Weissman, A. Z. Eisen, M. Lewis and M. Karon, Bethesda, Md. (introduced by Nathaniel $I$. Berlin).

The present studies were performed since quantitative estimates of pyrimidine production rates and utilization of salvage pathways by man have been lacking. 2- $\mathrm{C}^{14}$ or $6-\mathrm{C}^{14}$-orotic acid or uracil was given intravenously to patients with slowly growing malignancies and the specific activities of respiratory carbon dioxide, urinary uracil, and urinary pseudouridine (5-ribosyluracil) were followed. Subject to the assumption that orotic acid degradation occurred principally through initial conversion to pyrimidine nucleotides, calculation of the ratio of daily pseudouridine excretion to cumulative isotopic excretion in urinary pseudouridine gave estimates of pyrimidine nucleoside production in three subjects of between 1.1 and $1.6 \mathrm{~g}$ per day. Renal clearance of uracil exceeded 
$50 \mathrm{ml}$ per minute and plasma levels were estimated as less than $1 \mu \mathrm{g}$ per $\mathrm{ml}$. Little or no utilization of uracil for pyrimidine nucleotide synthesis occurred at normal plasma levels, since there was a rapid disappearance of radioactivity from plasma and urinary uracil and pseudouridine; thus, the potential salvage pathway of uracil metabolism is of no physiologic importance for most tissues.

Studies on Lysosomes. The Effect of Cortisone and Endotoxin. Gerald Weissmann and Lewis Thomas,* New York, N. Y.

Lysosomes are subcellular particles which release such acid hydrolases as acid phosphatase, $\beta$-glucuronidase, and cathepsins upon the death of cells. Previous work indicated that lysosomal enzymes were released from particles in vitro by treatment with vitamin $\mathrm{A}$ or by irradiation with the full mercury spectrum. Release of enzymes was significantly inhibited when suspensions were prepared from animals pretreated with glucocorticoids, while deoxycorticosterone acetate was ineffective. Because some of the effects of endotoxin in vivo might result from the antemortem release of lysosomal enzymes into cell sap or into the circulation, young rabbits were injected intravenously with Aerobacter aerogenes endotoxin (20 to $40 \mu \mathrm{g}$ ). Lysosome-rich suspensions from livers of these and control animals were prepared in $0.25 \mathrm{M}$ sucrose at various times after endotoxin administration. To test the "lability" of the particles, aliquots were irradiated with a mercury vapor lamp, while others were simply incubated at $37^{\circ} \mathrm{C}$. After treatment, suspensions were centrifuged at $15,000 \mathrm{G}$ for 20 minutes and the $\beta$-glucuronidase and catheptic activity of the supernatants determined. Five minutes after endotoxin injection, but not after 1 minute, depletion of the total enzyme content of the suspensions was observed, with increased lability of the particles to irradiation. Depletion of both enzymes reached maximum at 15 and 30 minutes after endotoxin $(66 \pm 12 \%$ of control total), and activity released into the supernatant of irradiated suspensions was increased over controls by $20 \pm 6 \%$ (cathepsins) and $48 \pm 12 \%$ ( $\beta$-glucuronidase). These effects lasted for 18 hours. Pretreatment of the animals with cortisone acetate $(50 \mathrm{mg} / \mathrm{kg})$ for 3 days inhibited the depletion of enzyme and reduced the lability of the particles to below control values. These experiments suggest that one action of endotoxin is to release acid hydrolases from particulate form within cells, and that glucocorticoids serve to stabilize such particles against injury by several agents.

Blood Viscosity Alterations in Dysproteinemia. Roe E. Wells, Jr. and Richard D. Perera, Boston, Mass. (introduced by Eugene C. Eppinger).

That quality of blood which describes either its viscosity or fluidity is dependent upon the concentration of the cellular elements and the forces creating the conditions of flow. Although plasma is known to have a relatively constant viscosity under most conditions of flow, it has been demonstrated that a suspension of red cells in plasma has a viscosity that is both higher and more flow-dependent (viscosity falling as flow rate rises) than a suspension of red cells in saline at equivalent hematocrit values. Present evidence suggests that globulin is the plasma protein component that accounts for the greater part of this effect. Studies of blood viscosity have been conducted in patients with abnormal elevations of serum globulins including those with macroglobulins. Two viscometers were used: one, a Couette type requiring a $5 \mathrm{ml}$ sample capable of recording shear stress and thereby viscosity over a shear rate range from 0.1 to $20 \mathrm{sec}^{-1}$; the second, a cone plate type viscometer requiring a $2 \mathrm{ml}$ sample and providing a shear rate range from 24 to $240 \mathrm{sec}^{-1}$. All samples were measured immediately after collection at $37^{\circ} \mathrm{C}$. Plasma or serum protein analyses were done by biuret and paper electrophoretic methods. Blood viscosity values of patients with elevated globulin levels were significantly higher than the values found in a large number of patients with normal serum proteins of equivalent hematocrit levels. Plasma viscosity values were also higher than those of normal subjects, and in the case of one patient with macroglobulinemia, plasma viscosity also demonstrated a significant degree of shear rate (flow) dependence.

\section{Chromatograms of Individual Achlorhydric Gastric} Juice Spcimens in Subjects With and Without Pernicious Anemia. J. D. Welsh, J. Hartzog, L. Russell And S. Wolf,* Oklahoma City, Okla.

Separation of the nondialyzable components of individual human gastric juice specimens on the IRC-50 Amberlite resin column has been shown to yield reproducible and comparable patterns. The purpose of this paper is to present data on the chromatograms of individual achlorhydric gastric juice specimens from patients with and without pernicious anemia, the alterations in such patterns when serum albumin is present, and, for comparison, the paper electrophoretic patterns on the same samples. Separate chromatograms were performed on the individual stimulated gastric juice specimens from eight subjects with pernicious anemia. The patterns were distinct from those of stimulated normals, containing only four of the usual six protein peaks. The two proteolytic peaks were missing from all specimens. Four of the specimens contained serum albumin. This could be localized in the last three protein peaks, which in turn were unusually large. Paper electrophoresis of the same specimens also revealed a characteristic pattern for pernicious anemia, including the identification of serum albumin in the gastric juice, confirming the work of Glass. A similar "pernicious anemia" pattern on resin column chromatography or electrophoresis could be identified in the fasting juice of an occasional subject without pernicious anemia when no hydrochloric acid was present. Even serum albumin was demonstrated in the fasting achlorhydric juice from one patient without pernicious anemia. After adequate stimulation, however, 
the specimens of such patients yielded a normal chromatogram and a normal pattern on paper electrophoresis. Serum albumin was no longer identified. In contrast, the gastric juice patients with pernicious anemia after stimulation yielded the same abnormal pattern as that encountered in the fasting juice and distinctly different from the normal.

The Metabolism of Ring-labeled L-Thyroxine-C $C^{\mathbf{1}}$ in Rat, Dog, and Man. C. D. West, L. F. Kumagai and M. J. Gortatowski, Salt Lake City, Utah (introduced by Frank H. Tyler).

In order to investigate the metabolic fate of the thyronine ring of thyroxine, L-thyroxine- $\mathrm{C}^{14}(1.20 \mathrm{mc} / \mathrm{mmole})$, with the radioisotope in the phenolic ring, was prepared from uniformly labeled $\mathrm{C}^{14}$-phenol. The synthetic product was proven to be L-thyroxine by mixed melting point, optical rotation, paper chromatography, and bioassay. When it was given intraveneously to intact rats, a total of $67 \%$ of the administered radioactivity was excreted in feces in 12 days with $87 \%$ of the fecal excretion occurring during the first 3 days. Twenty per cent was excreted in urine with a half-time $\left(\mathrm{t}_{1}\right)$ of 51 hours, and less than $0.1 \%$ appeared as respiratory $\mathrm{C}^{14} \mathrm{O}_{2}$. In man the total amount of radioactivity excreted in the urine was comparable to that observed in the rat, but the rate of excretion was slower $\left(t_{\frac{1}{2}}=120 \mathrm{hrs}\right)$. In the dog total urinary excretion was considerably less than in either man or rat and much faster $\left(t_{1}=24 \mathrm{hrs}\right)$. The dog also differed from man in that the rate of removal of the injected L-thyroxine- $\mathrm{C}^{14}$ from the blood stream was much more rapid. In rats with their common bile ducts catheterized, the per cent of administered $\mathrm{C}^{14}$ excreted in bile varied from 57 to $80 \%$ as a direct function of the weight of thyroxine administered. Paper chromatography revealed that $\mathrm{C}^{\mathbf{1 4}}$-thyroxine glucuronide and $\mathrm{C}^{14}$-thyroxine made up approximately $90 \%$ of the biliary radioactivity with the remaining $10 \%$ in an unidentified highly polar compound. The ratio of $\mathrm{C}^{\mathbf{1 4}}$-thyroxine glucuronide in bile was approximately 1.0 when large amounts of thyroxine were administered but decreased considerably with smaller doses. $\mathrm{C}^{\mathbf{1 4}}$-thyroxine glucuronide was identified in the urine by paper chromatography, but over $75 \%$ of the administered radioactivity was in three compounds which have not yet been identified. Neither $\mathrm{C}^{\mathbf{1 4}}$-thyroxine nor $\mathrm{C}^{\mathbf{1 4}}$-triiodothyronine was found.

A Demonstration of the Dynamic Nature of Hypertrophic Subvalvular Aortic Stenosis. RoBert E. Whalen, Allan I. Cohen, Robert G. Sumner and Henry D. McIntosh, Durham, N. C. (introduced by William M. Nicholson).

Patients with genetic, clinical, $\mathrm{X}$-ray, and electrocardiographic features suggesting hypertrophic subvalvular aortic stenosis have been studied in this and other laboratories. When no pressure gradient is demonstrated across the outflow tract of the left ventricle, the diagnosis of idiopathic cardiac hypertrophy is usually entertained. Such a patient was studied on two occasions and while in a basal state was found to have no significant gradient between the left ventricle and the systemic artery. However, on both occasions, during an infusion of isoproterenol $(1.2 \mu \mathrm{g} / \mathrm{min})$, this patient developed an $80 \mathrm{~mm}$ $\mathrm{Hg}$ systolic gradient at the subvalvular level. (The subvalvular location of the obstruction was conclusively demonstrated by "pull through" tracings). A smaller but definite gradient also developed during and after exercise. Indicator dilution curves obtained when the gradient was presented indicated considerable mitral insufficiency, a feature commonly present in patients with hypertrophic subvalvular aortic stenosis. Similar curves obtained when there was no gradient failed to reveal mitral insufficiency. Repetition of the study in a group of patients with left ventricular hypertrophy from other causes, including aortic valvular stenosis, did not produce a similar response. A study of laboratory animals with left ventricular hypertrophy (turkeys, heart-block dogs, and greyhounds) also failed to demonstrate such a response to isoproterenol. The inotropic effect of isoproterenol presumably leads to a disproportionate strengthening or asynchronous contraction of the left ventricular outflow tract. These changes result in hemodynamic findings similar to those observed in patients with overt hypertrophic subvalvular aortic stenosis. It is suggested that similar studies may further elucidate the basic defect present in patients with this syndrome, and that isoproterenol infusion may serve as a provocative test for the detection of patients predisposed to develop hypertrophic subvalvular aortic stenosis.

Observations in Experimental Magnesium Depletion. R. Whang and L. G. Welt,* Chapel Hill, N. C.

Sprague-Dawley rats were fed a diet free of sodium, potassium, chloride, phosphorus, and magnesium. Control groups were gavaged with adequate quantities of these ions, whereas experimental groups were gavaged with identical solutions devoid of magnesium. The observations were as follows: magnesium-deficient animals had hypomagnesemia, hypercalcemia, azotemia, an expanded "chloride space" in muscle, and a significant decrease in muscle potassium. This deficit in muscle potassium could not be avoided despite the administration of as much as 4 mmoles $\mathrm{K}$ per day throughout the study. In another study identical in description, except that neither control nor experimental animals received potassium, it was demonstrated that from the seventh day on, the excretion of potassium was significantly greater in the magnesiumdeficient group. This larger urinary excretion of potassium and the depletion of muscle potassium obtained in magnesium-deficient animals, despite the fact that the serum levels of potassium were the same in the control and experimental groups. These data strongly suggest that magnesium deficiency (or the associated hypercalcemia) interferes with the ability of muscle cells to maintain an appropriate concentration gradient for potassium between cellular and extracellular water. 
Specificity in Fatty Acid Esterification during Fat Absorption. H. Malcolm Whyte, Arthur Karmen and DeWitt S. Goodman, Bethesda, Md. (introduced by Robert S. Gordon, Jr.).

The fatty acid specificity of several different rat intestinal esterifying systems has been studied during fat absorption. Mixtures containing equal amounts of $\mathrm{C}^{14}$-labeled palmitic, oleic, and linoleic acids, but with varying ratios of unlabeled fatty acids, were given by gastric intubation to rats with cannulated thoracic ducts. The chylomicron lipid so obtained was chromatographed on silicic acid columns to separate glycerides (mostly triglycerides), cholesterol esters, and lecithin. Gasliquid chromatography was then employed to measure the mass, total radioactivity, and specific radioactivity of the individual fatty acid components of these three fractions. These data provided quantitative information on the specificity of incorporation of each of the three fatty acids into each lipid class, and on the relative extent to which each fatty acid in each fraction was diluted with endogenous fatty acid during absorption into the chyle. All three exogenous fatty acids were incorporated into the glycerides without preference, but with evident endogenous dilution. Oleic acid was clearly preferred for cholesterol esterification; palmitic and linoleic acids were used to an equivalent extent although less than oleic acid. In contrast, there was marked discrimination against oleic acid and slight selectivity for linoleic acid in the lecithin fraction. Dilution of the fed acids with endogenous fatty acids was greater in the cholesterol ester and lecithin fractions than in the glycerides; this was particularly true for palmitic acid. In addition, there were dramatic differences in the positional specificities of the different fatty acids in lecithin; these did not occur in triglycerides.

Studies on the Two Pathways of Cholesterol Synthesis. Jean D. Wilson, Dallas, Tex. (introduced by M. D. Siperstein).

It has recently been demonstrated that the completion of cholesterol synthesis occurs by two separate pathways. Increased activity of either of these two pathways may be responsible for derangements in cholesterol metabolism. One pathway (I) involves the completion of the sterol nucleus prior to saturation of the side chain (lanosterol $\rightarrow$ zymosterol $\rightarrow$ desmosterol $\rightarrow$ cholesterol). A second pathway (II) of cholesterol synthesis has been described in a tumor of the mouse preputial gland in which the sterol side chain is reduced prior to completion of the nucleus (lanosterol $\rightarrow$ dihydrolanosterol $\rightarrow \Delta^{8}$-methostenol $\rightarrow \Delta^{7}$-methostenol $\rightarrow \Delta^{7}$-cholestenol $\rightarrow$ cholesterol). The following study was undertaken to devise a means for determining which of the pathways is present in normal tissues. Triparanol is known to block the reduction of the 24-25 double bond in pathway I, as a result of which desmosterol accumulates in serum and tissues. Since pathway II also requires reduction of this double bond (lanosterol $\rightarrow$ dihydrolanosterol), then lanosterol would accumulate in tissues which contain pathway II if triparanol blocks the reduction of all 24-dehydrosterols. Utilizing both silicic acid and gas-liquid chromatographic techniques, the preputial gland sterols from rats fed triparanol were analyzed; three major components were found and identified as cholesterol, desmosterol, and lanosterol, whereas untreated preputial gland contains predominantly cholesterol, $\Delta^{\tau}$-cholestenol, and $\Delta^{\tau}$-methostenol. The accumulation of lanosterol in response to triparanol treatment constitutes strong evidence that the preputial gland does in fact contain pathway II. Of the remaining organs of the rat which have been similarly analyzed, only skin appears to contain a second pathway of major significance. In summary: 1) A technique has been devised to distinguish the presence and contribution of the two biosynthetic pathways of cholesterol synthesis in living tissues ; 2) evidence is advanced that in at least two tissues of the normal rat cholesterol can be formed via both synthetic pathways.

Achievement of Homografts with Selective Lymphatic Irradiation.. H. S. Winchell AND M. Pollycove, Berkeley, Calif. (introduced by John H. Lawrence).

The homograft rejection response appears to be a function of the lymphatic tissues. Successful homografting requires the suppression of this function. We have developed a method for delivering radiation more selectively to lymphatic structures than heretofore has been available for the purpose of preparing large mammals, such as dogs and man, for tissue grafting. Yttrium ${ }^{20}$ chelated by diethylenetriamine pentaacetic acid (DTPA) was administered intravenously to dogs after which the dog's urine, which contained the rapidly excreted $\mathrm{Y}^{90}$-DTPA, was continuously pumped into one of its veins for a period of 6 hours. This procedure allowed for maintenance of constant levels of radioactivity in the body during the time of intravenous urine cycling and for rapid clearance of radioactivity from the body of the dog when intravenous urine cycling was stopped. Dogs given sublethal doses of $\mathrm{Y}^{\infty}$-DTPA showed selective destruction of lymphocytes in both the circulating blood and on histologic examination of lymph nodes, without concomitant depression of platelets, granulocytes, or reticulocytes in the circulating blood. Lethally irradiated dogs showed depression of platelets, granulocytes, and reticulocytes with virtual absence of lymphocytes in the circulating blood. The lives of the lethally irradiated animals could be saved by the administration of autologous or homologous bone marrow with evidence of proliferation of the marrow graft in both instances. A series of renal homografts in the lethally irradiated dogs treated with homologous bone marrow gave evidence of induced "compatibility" of the host and donor tissues. One animal had a renal homograft from an unrelated donor which produced urine for a 34-day period. Preliminary evidence obtained in the treatment of human acute lymphatic leukemia indicates that this procedure can effect relatively selective depression of lymphocytes in man as well as in dogs. 
Back-diffusion of $\mathrm{NH}_{3}$ : The Nephron's Terminal $H^{+}$-generating System. KeNNETH A. WOEBER AND A. Gorman Hills,* Miami, Fla.

Urinary (total) ammonia arises from enzymatic deaminations within certain renal tubular cells. The dependence of its rate of excretion upon urine $\mathrm{pH}$ suggests diffusion of $\mathrm{NH}_{3}$ (but not $\mathrm{NH}_{4}^{+}$) into tubular fluid at or below the site of $\mathrm{H}^{+}$secretion. Acute experiments in three healthy men show that 1) the logarithmic rate of urinary ammonia excretion is a direct linear function (about $0.018 \mu \mathrm{Eq} / \mathrm{ml}$ ) of urine flow throughout its physiologic range when urine $\mathrm{pH}$ is held constant at any value. In the same experiments, provided urine $\mathrm{pH}$ is $<6.0,2)$ the logarithmic rate of titratable acid excretion is an inverse linear function of flow, and 3) the slope of this regression (sign ignored) is similar. In urines of higher $\mathrm{pH} 4) \mathrm{CO}_{2}$ tension $\left(\mathrm{pCO}_{2}\right)$ varies inversely with flow. These observations afford indirect but persuasive evidence for the following formulation of the nonenzymatic determinants of urinary ammonia excretion: 1) diffusion of $\mathrm{NH}_{3}$ to equilibrium in distal tubular fluid; 2) subsequent back-diffusion of $\mathrm{NH}_{3}$ varying as a function of facultative water reabsorption, and entailing 3) intraluminal conversion of $\mathrm{NH}_{4}^{+}$to $\mathrm{NH}_{3}$ with $\mathrm{H}^{+}$ generation. The value for the regression gives a biased estimate of variation of the system's activity with changing urine flow, for experimental conditions required to stabilize urine $\mathrm{pH}$ doubtless affect distal tubular $\mathrm{H}^{+}$secretion. A better preliminary estimate, yielded by experiments that were similar except that urine $\mathrm{pH}$ was permitted to vary, is $0.0155 \log \mu \mathrm{Eq}$ per $\mathrm{ml}$. This system is clearly the major cause for the high $\mathrm{pCO}_{2}$ of alkaline urine, and its extra $\mathrm{H}^{+}$-generation at low urine flows is ample to explain the correspondingly increased $\mathrm{pCO}_{2}$.

Humoral Factors in Transplantation Immunity. PAUL L. Wolf, Chiyo Chiba, Hernanio Ramos, Peter Schollmeyer and R. J. Bing,* Detroit, Mich.

Previous observations in this laboratory during the course of 250 canine cardiac homograft experiments revealed that in the second-set graft transplants a rapid exudative pericarditis and mural endocarditis occurred within 15 minutes after grafting. To demonstrate the presence of humoral factors responsible for rapid rejection, two types of experiments were performed. In the first series, first- and second-set canine cardiac transplants were perfused with radioiodinated serum albumin. Increased radioactivity of the tissue was present in the grafts 15 minutes after transplantation, especially in the second-set hearts, demonstrating increased vascular permeability. No cellular infiltrate was present. In the second group of experiments, humoral antibodies were demonstrated in vitro. Canine hearts from Dog I were perfused with Dog I's own blood. To this was added ultracentrifuged cell-free serum from a second dog (Dog II) which had been previously sensitized with spleen cells from Dog I. Radioactive iodinated serum albumin was added 1 hour after perfusion was begun. Increased radioactivity was found in the tissue of all 16 grafts. Two grafts demonstrated a minimal immune-type granulomatous myocarditis. The results suggest the presence of humoral factors contributing to homograft rejection since increased vascular permeability appears immediately after exposure of grafts to sensitized cell-free plasma, and graft rejection occurs without the presence of cellular infiltrate.

The Role of Ion Size in Thyroidal Anion Transport. J. WolfF* and J. R. Maurey, Bethesda, Md.

Iodide ion is actively concentrated by thyroid tissue whereas $\mathrm{Br}^{-}$ion is poorly concentrated and $\mathrm{Cl}^{-}$and $\mathrm{F}^{-}$ ions not at all. The possibility of a size requirement for anion transport by thyroid tissue was therefore investigated in sheep thyroid slices. Steady state $\mathrm{K}_{\mathbf{M}}$ values, as well as $K_{I}$ values for iodide transport, were determined for anions such as $\mathrm{I}^{-}, \mathrm{SCN}^{-}, \mathrm{ClO}_{4}^{-}, \mathrm{NO}_{3}^{-}, \mathrm{BF}_{4}^{-}, \mathrm{ReO}_{4}^{-}$, and so forth. Where not available from the literature, partial molal ionic volumes of these anions were determined from the densities of their solutions according to the Masson relation. It was found that, despite the different shapes of the anions, the $\mathrm{pK}$ values for anion transport increased as a linear function of the partial molal ionic volume of the ion. A maximum $\mathrm{pK}$ was reached at anion volumes between 45 and $48 \mathrm{ml}$ per mole. Beyond this volume $\mathrm{pK}$ values again decreased. Comparison with other size parameters (e.g., from crystal or conductance measurements) showed no correlation with $\mathrm{K}_{\mathbf{M}}$ or $\mathrm{K}_{\mathbf{I}}$ values of anion transport. It is concluded that univalency and a suitable size (as measured by the partial molal ionic volumes) describe minimum, but not necessarily sufficient, requirements for anion transport into thyroid tissue.

Hypogammaglobulinemia Produced by the Administration of 6-Thioguanine to Patients with Nephrosis. Sheldon M. WolfF and Howard C. Goodman, Bethesda, Md. (introduced by James H. Baxter).

During a study of the effect of 6-thioguanine (6-TG) on the idiopathic nephrotic syndrome, we have observed a marked decrease in serum $\gamma$-globulin (paper electrophoresis). Prednisone therapy alone was not associated with a significant change in $\gamma$-globulin in four of five patients. Three of this group and an additional "steroidresistant" patient were treated with 6-TG alone or 6-TG plus prednisone. In the two patients who received 6-TG alone $\gamma$-globulin fell from 624 to $160 \mathrm{mg}$ per $100 \mathrm{ml}$ and from 506 to $356 \mathrm{mg}$ per $100 \mathrm{ml}$ (first 2 weeks of therapy). In the three patients treated with prednisone plus 6-TG marked falls in $\gamma$-globulin levels of 83,82 , and $144 \mathrm{mg}$ per $100 \mathrm{ml}$, respectively, occurred in all, although two had previously shown no fall in $\gamma$-globulin with prednisone alone. Decreases in $\gamma$-globulin occurred despite diminution or disappearance of proteinuria. Gel electrophoresis and immunoelectrophoresis confirmed the fall in 
$\gamma$-globulin and suggested concomitant decrease in the $\beta_{2} \mathrm{M}(\gamma-1 \mathrm{M})$ and $\beta_{2} \mathrm{~A}(\gamma-1 \mathrm{~A})$ globulins. Isohemagglutinin titers fell from $1: 256$ to $1: 32$ during 6-TG therapy in one patient. The use of 6-TG has been associated with clinical remission in one patient (relatively steroid-resistant), with improvement in two, and no response in one (steroid-resistant patients). Temporary bone marrow suppression occurred in all 6-TG-treated patients, including two severe episodes. Plasma cells were present on bone marrow examination at the end of 6-TG therapy. The above data demonstrate the capacity of 6-TG to decrease the serum globulins with which antibody activities have been associated, and raise the question of a possible correlation between the production of hypogammaglobulinemia and clinical improvement.

Detection and Quantitation of Left-to-Right Shunts by an Oximetric Inert Gas Technic. ROBERT C. WoOD AND Hiram W. Marshall, Rochester, Minn. (introduced by Earl H. Wood).

In the presence of a left-to-right shunt, continuous recording of the oxygen saturation of pulmonary arterial blood during a sudden transient change from breathing air to breathing a gas mixture containing no oxygen will reveal a decrease in oxygen saturation of pulmonary arterial blood that occurs simultaneously with that in systemic arterial blood. This must result from an intracardiac left-to-right shunting of the reduced hemoglobulin, caused by transient reduction in oxygen tension in the lungs. The reliability and sensitivity of this inert gas technic in relation to the highly sensitive but technically more difficult method of venous dye-dilution curves were studied in six dogs having chronic atrial septal defects. The magnitude of the left-to-right shunts varied from 9 to $65 \%$. No left-to-right shunt was detected in two dogs by conventional blood oxygen-saturation methods, although these shunts were demonstrable by both venous dye-dilution and inert gas curves. Maximal sensitivity was attained when the animals were hyperventilated at a rapid rate with $10 \%$ oxygen and $5 \%$ carbon dioxide in helium or nitrogen before and after a transient change (two to six breaths) to ventilation with $5 \%$ carbon dioxide in helium or nitrogen. Correlation of the magnitude of the left-to-right shunts by venous dye-dilution and inert gas curves was comparable to that for venous dye-dilution and blood oxygen-saturation methods. Experience with detection and quantitation of left-to-right shunts with these methods in patients has been similar. The need for only a single catheter and the fact that no added electronic equipment is necessary are important practical advantages of the inert gas method.

Studies on the Antigenicity of Natural and Synthetic Polynucleotides. Stanley Yachnin, Chicago, Ill. (introduced by Theodore N. Pullman).

Rabbits were immunized with virtually protein-free polyadenylic acid (Poly A), polycytidylic, polyuridylic, and polyinosinic acids, rat, guinea pig, and rabbit liver ribonucleic acids (RNA) deoxyribonucleic acids (DNA), and ribosomes. Search for antibody to polynucleotides by gel diffusion, complement fixation, and immunochemical precipitin techniques was negative. Previous workers have demonstrated the formation of soluble complexes between DNA and bovine serum albumin (BSA) under conditions of controlled $\mathrm{pH}$ (5.0 to 6.0) and low ionic strength capable of protecting BSA from heat coagulation. Similarly, soluble complexes were formed between heat-denatured BSA (HBSA)-Poly A and HBSA-RNA. Analysis of antisera formed to these complexes by the Ouchterlony technique showed precipitin lines which were absent when antisera reacted with native BSA or HBSA. Absorption of these antisera with HBSA removed most precipitating antibody; a small amount of antibody remained, precipitable only by polynucleotide-HBSA complex. Precipitin curves with antisera to polynucleotide-HBSA complexes, native BSA, and HBSA, using all three antigens, showed that in all three instances at equivalence homologous antigen precipitated by far the greatest amount of antibody. Homologous polynucleotide alone was incapable of: 1) precipitating antibody from homologous anti-polynucleotide-HBSA serum; 2) precipitating antibody from homologous antiserum absorbed with HBSA; 3 ) reducing the amount of antibody precipitable from antipolynucleotide-HBSA serum. It is concluded that: 1) synthetic and mammalian polynucleotides are not antigenic in rabbits; 2) antibody to mammalian liver ribosomes will not react with protein-free homologous RNA; and 3) antibody formed to RNA- or Poly A-HBSA complexes differs from that formed to native BSA or HBSA, but that polynucleotide alone acts neither as hapten nor as antigen. It is likely that the heat denaturation of BSA in the presence of polynucleotide confers upon it antigenic properties which are not present in native BSA or in BSA heat-denatured in the absence of polynucleotide. 